\title{
UICN
}

Lignes directrices pour l'application des catégories de gestion aux aires protégées

Édité par Nigel Dudley

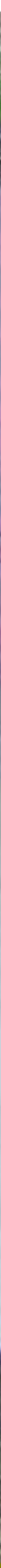




\section{Lignes directrices pour l'application des catégories de gestion aux aires protégées}




\section{UICN}

Fondée en 1948, l'UICN (Union internationale pour la conservation de la nature) rassemble des Etats, des organismes publics et un large éventail d'organisations non gouvernementales au sein d'une alliance mondiale unique: plus de 1000 membres dans quelque 160 pays. L'UICN, en tant qu'Union, a pour mission d'influer sur les sociétés du monde entier, de les encourager et de les aider pour qu'elles conservent l'intégrité et la diversité de la nature et veillent à ce que toute utilisation des ressources naturelles soit équitable et écologiquement durable. Afin de sauvegarder les ressources naturelles aux plans local, régional et mondial, l'UICN s'appuie sur ses membres, réseaux et partenaires, en renforçant leurs capacités et en soutenant les alliances mondiales.

Website: www.iucn.org

\section{La Commission Mondiale pour les Aires Protégées (CMAP)}

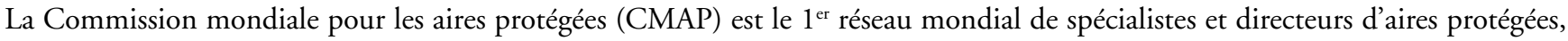
comprenant plus de 1300 membres dans 140 pays. La CMAP est une commission volontaire de l'UICN parmi 6 autres. Elle est administrée par le Programme des Aires Protégées situé au Siège mondial/Secrétariat de l'UICN à Gland, Suisse.

La mission de la CMAP est de promouvoir et de gérer efficacement un réseau mondial représentatif d'aires protégées terrestres et marines, faisant partie intégrante de la mission de l'UICN.

Website: www.iucn.org/themes/wcpa

\section{Conseil Régional pour l'Environnement de la Junta de Andalucia}

Le Conseil Régional pour l'Environnement de la Junta de Andalucia est l'agence responsable du gouvernement régional d'Andalousie pour la conservation de la nature, l'application des règlements environnementaux sur la façon d'utiliser les ressources naturelles, la déclaration et la gestion des aires protégées ainsi que la définition, le développement et l'implantation de la stratégie et des règles de mitigation et d'adaptation en matière de changement climatique.

\section{Fundación Biodiversidad}

La Fundación Biodiversidad (Fondation Biodiversité) est une fondation sans but lucratif, fondée en 1998 en suite des engagements assumés par l'Espagne après la ratification de la Convention sur la Diversité Biologique. Son activité se développe dans le domaine de la conservation, de l'étude et de l'utilisation durable de la biodiversité, ainsi que la coopération internationale pour le développement. Par la Coopération Internationale, la Fundación Biodiversidad réussit unir des efforts et créer des synergies, en plus de promouvoir la collaboration avec des organismes, institutions et programmes nationaux et internationaux. 


\section{Lignes directrices pour l'application des catégories de gestion aux aires protégées}

Édité par Nigel Dudley 
La terminologie géographique employée dans cet ouvrage, de même que sa présentation, ne sont en aucune manière l'expression d'une opinion quelconque de la part de l'UICN ou des autres organisations concernées sur le statut juridique ou l'autorité de quelque pays, territoire ou région que ce soit, ou sur la délimitation de ses frontières.

Les opinions exprimées dans cette publication ne reflètent pas nécessairement celles de l'UICN ou des autres organisations concernées.

L'UICN et les autres organisations concernées rejettent toute responsabilité en cas d'erreurs ou d'omissions intervenues lors de la traduction en français de ce document dont la version originale est en anglais.

\section{Publié par:}

Droits d'auteur:

\section{Citation:}

ISBN:

\section{Traduction:}

Photos couverture:

Mise en page:

Produit par:

Imprimé par:

Disponible auprès du:
UICN, Gland, Suisse

C2008 Union internationale pour la conservation de la nature et de ses ressources

La reproduction de cette publication à des fins non commerciales, notamment éducatives, est permise sans autorisation écrite préalable du détenteur des droits d'auteur à condition que la source soit dûment citée.

La reproduction de cette publication à des fins commerciales, notamment en vue de la vente, est interdite sans autorisation écrite préalable du détenteur des droits d'auteur.

Dudley, N. (Éditeur) (2008). Lignes directrices pour l'application des catégories de gestion aux aires protégées. Gland, Suisse : UICN. $\mathrm{x}+96 \mathrm{pp}$.

978-2-8317-1087-7

Jacqueline et Jean-Pierre d'Huart

Devant : Discussion avec des communautés locales près de Morondava, Madagascar, au sujet du zonage d'une aire protégée qui devrait préserver une espèce rare de baobab. (C) Nigel Dudley

Dos : Nouvelle Calédonie (C Dan Laffoley

Bookcraft Ltd, Stroud, Royaume-Uni

Service des publications de l'UICN

Page Bros, Norwich, Royaume-Uni

UICN (Union internationale

pour la conservation de la nature)

Service des publications

Rue Mauverney 28

1196 Gland

Suisse

Tél +41229990000

Fax +41229990020

books@iucn.org

www.iucn.org/publications

Il existe aussi un catalogue des publications de l'UICN. 


\section{Table des matières}

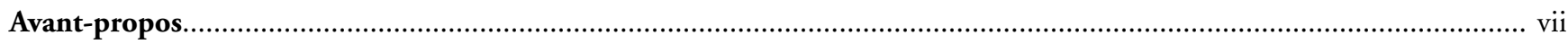

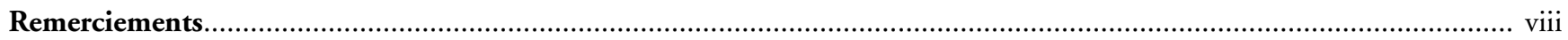

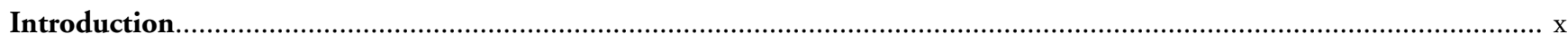

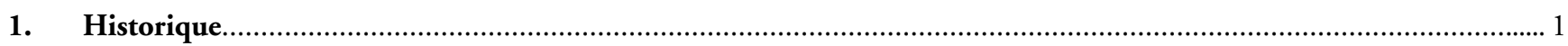

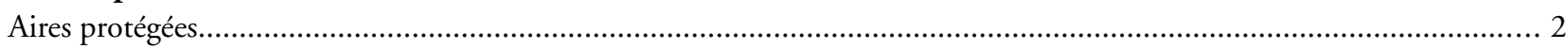

Historique des catégories d'aires protégées de l'UICN ..................................................................................... 4

Raison d'être des catégories de gestion des aires protégées de l'UICN ...............................................................6

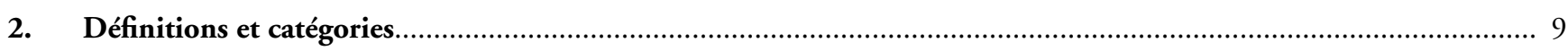

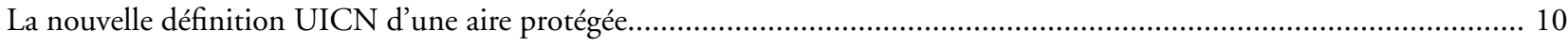

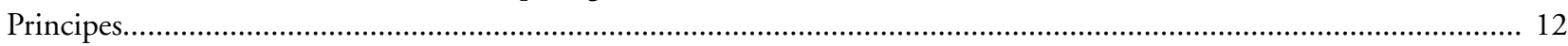

Définition d'un système d'aires protégées et l'approche par écosystème................................................................. 12

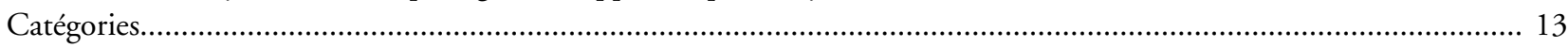

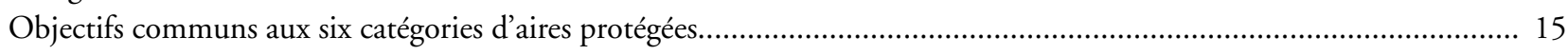

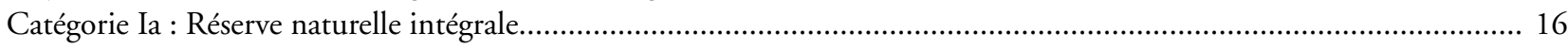

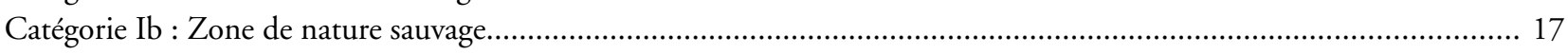

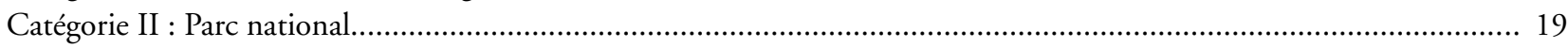

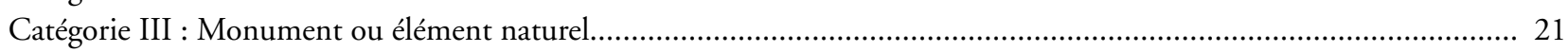

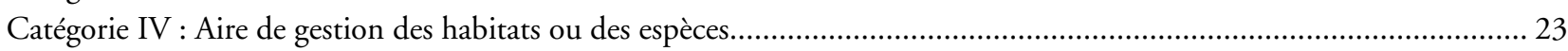

Catégorie V : Paysage terrestre ou marin protégé ............................................................................................... 25

Catégorie VI : Aire protégée avec utilisation durable des ressources naturelles......................................................... 27

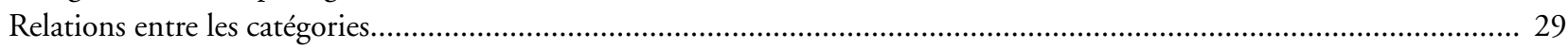

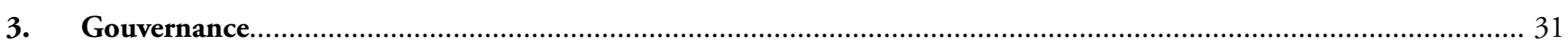

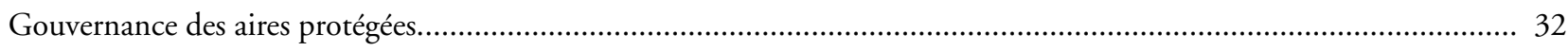

Gouvernance par les populations autochtones et les communautés locales............................................................... 35

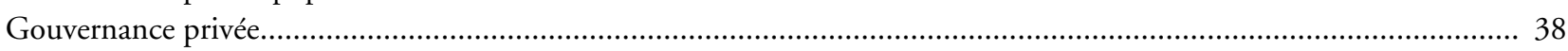

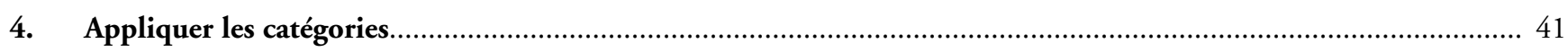

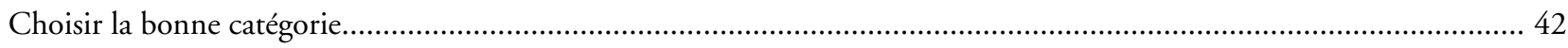

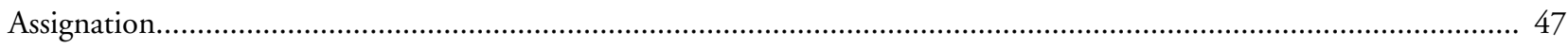

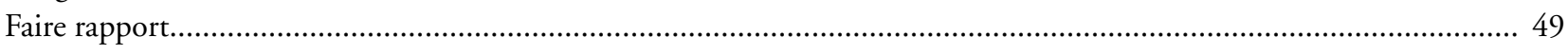

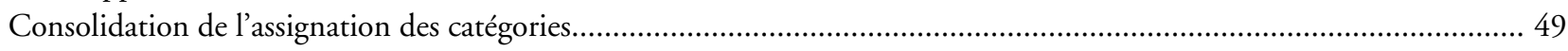

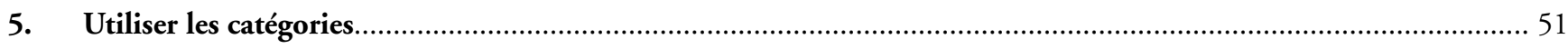

Utiliser les catégories de l'UICN comme un instrument de planification de la conservation....................................... 52

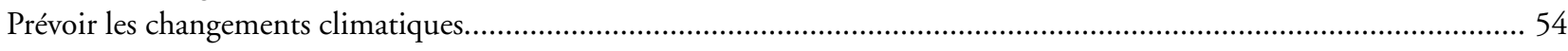

Utiliser les catégories d'aires protégées de l'UICN comme un instrument de politique de la conservation..................... 56

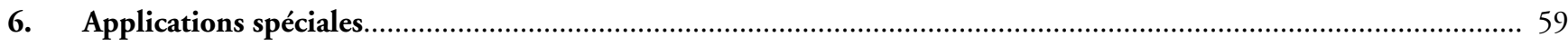

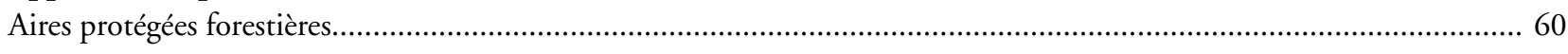

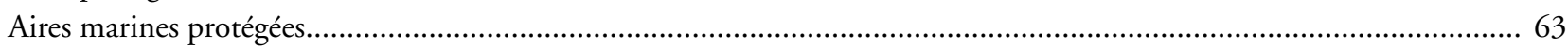

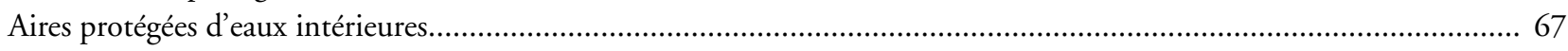

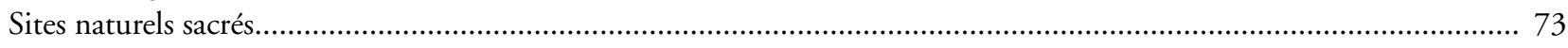

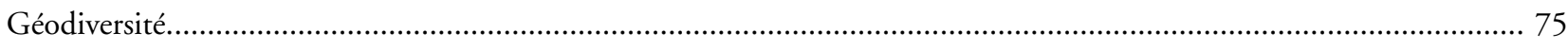

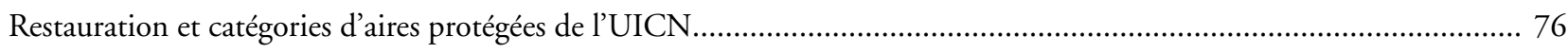

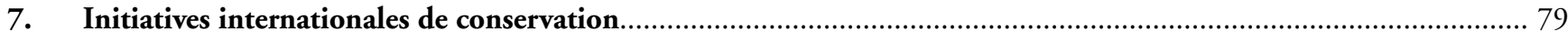

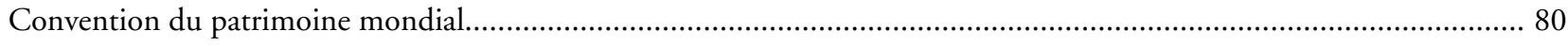

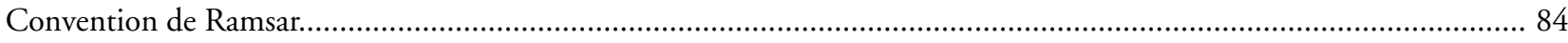

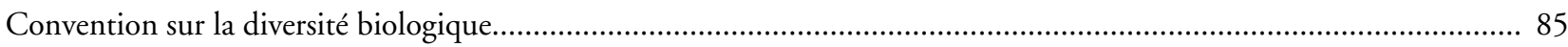




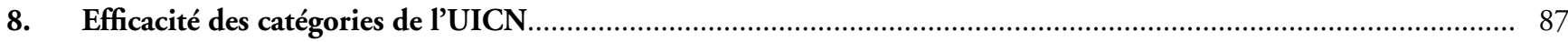

Evaluation de la gestion et les catégories de l'UICN ............................................................................................ 88

Annexe. Typologie et glossaire........................................................................................................................... 91

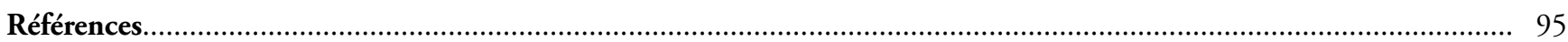

Tableaux

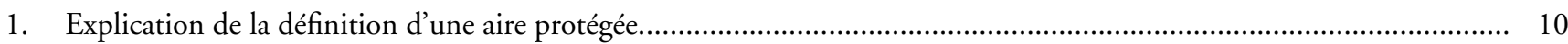

2. « Parcs nationaux » dans diverses catégories..................................................................................................... 14

3. «La matrice UICN des aires protégées » : un système de classification des aires protégées comprenant la

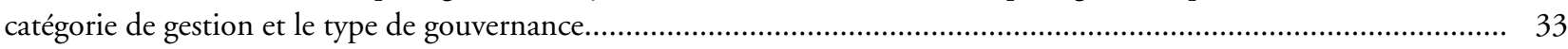

4. Comment la taille d'une aire protégée est liée à la catégorie....................................................................................

5. Analyse forces-faiblesses-opportunités-menaces pour les catégories face aux changements climatiques........................... 55

6. Exemples d'aires protégées forestières, ainsi que de forêts bien conservées qui ne sont pas des aires protégées

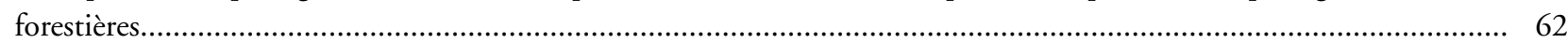

7. Distinguer les aires de conservation de la connectivité telles que les corridors, les étapes-relais biologiques et

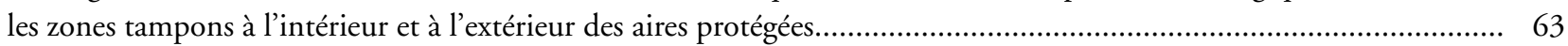

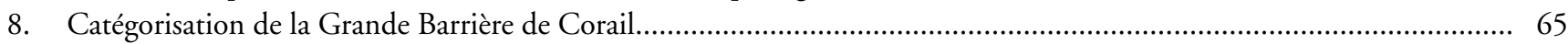

9. Application des catégories dans les aires marines protégées..................................................................................... 66

10. Exemples d'aires protégées de différentes catégories fournissant des bénéfices aux eaux intérieures................................. 70

11. Compatibilité de diverses stratégies de protection des eaux intérieures avec les catégories de l'UICN............................ 71

12. Les catégories d'aires protégées les plus appropriées pour différents types d'écosystèmes d'eaux intérieures...................... 72

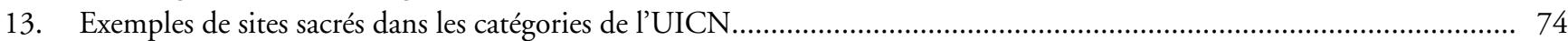

14. Exemples de géodiversité dans différentes catégories UICN d'aires protégées .............................................................. 76

15. Indications des catégories UICN appropriées pour différents aspects de la géodiversité.................................................. 76

16. Guide indicatif pour la restauration dans différentes catégories UICN.................................................................... 77

17. Evolution de la relation entre sites naturels du patrimoine mondial et aires protégées................................................. 81

18. Eléments du cadre de la CMAP pour évaluer l'efficacité de gestion d'aires protégées................................................... 88

19. Définitions des termes utilisés dans les lignes directrices........................................................................................ 91

\section{Figures}

1. Caractère naturel et catégories d'aires protégées de l'UICN .................................................................................... 29

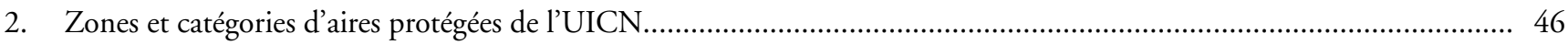

3. Processus pour assigner des catégories aux aires protégées......................................................................................... 49

4. Fréquence de l'occurrence des catégories d'aires protégées de l'UICN dans les sites naturels de biodiversité et de non-biodiversité du PM 


\section{Avant-propos}

Les aires protégées restent les constituants fondamentaux de pratiquement toutes les stratégies de conservation nationales et internationales, avec le soutien de gouvernements et d'institutions internationales telles que la Convention sur la diversité biologique. Elles sont au cœur des efforts réalisés pour protéger les espèces menacées dans le monde et, c'est de plus en plus reconnu, elles sont des fournisseurs essentiels de services écosystémiques et de ressources biologiques. Elles sont des éléments clés des stratégies pour atténuer les changements climatiques et, dans certains cas, elles servent aussi de véhicules pour protéger des communautés humaines menacées ou des sites de grande valeur culturelle ou spirituelle. Couvrant près de 12 pour cent de la surface terrestre, le système mondial des aires protégées représente un engagement unique envers l'avenir ; c'est un signe d'espérance dans ce qui semble parfois un glissement déprimant vers un déclin environnemental et social.

Les aires protégées ne sont pourtant en aucune façon des entités uniformes ; elles répondent à des objectifs de gestion très variés et sont dirigées par de nombreuses et diverses parties prenantes. D'un côté se trouvent des sites si importants et si fragiles que personne n'est autorisé à y pénétrer, alors que d'autres aires protégées englobent des paysages traditionnels, terrestres et marins, habités, où les activités humaines ont façonné des paysages culturels dont la biodiversité est très élevée. Certains sites appartiennent et sont gérés par des gouvernements, d'autres par des particuliers, des sociétés, des communautés ou des groupes religieux. Nous commençons à réaliser qu'il existe une bien plus grande variété de modes de gouvernance que nous ne l'avions pensé jusqu'ici.

Les catégories de gestion d'aires protégées de l'UICN sont un cadre mondial, reconnu par la Convention sur la diversité biologique, qui permet de classer la grande variété de types de gestion des aires protégées. Concentrer la gamme presque infinie d'approches en six catégories ne pourra jamais être plus qu'une approximation. Mais le grand intérêt et la passion qui sous-tendent le débat sur la révision de ces catégories montrent que pour de nombreux conservationnistes, entre autres, elles représentent un cadre global critique qui aide à façonner la gestion et les priorités des aires protégées dans le monde entier.

Cette révision ne s'est pas faite dans la précipitation. Elle a commencé par un projet de recherche consultatif de deux ans qui a présenté son rapport au Congrès mondial de la nature, à Bangkok, en 2004, et qui a abouti à une résolution qui demandait la production des lignes directrices présentées dans ce livre. Au cours des années qui ont suivi, l'UICN a consulté un très grand nombre de ses membres lors de réunions spéciales, de conférences, par des débats électroniques et par ce qui a pu sembler, parfois, un échange sans fin de correspondance. Nous sommes bien conscients que le résultat n'est pas parfait - ce serait impossible - mais nous croyons que l'interprétation de la définition d'une aire protégée et des catégories présentée ici représente l'opinion d'une grande majorité des membres de l'UICN. Il est important de remarquer qu'elle est complétée par les types de gouvernance de l'UICN, montrant par là même l'importance que l'Union accorde aux questions de gouvernance.

Au cours des années à venir, nous allons nous efforcer de promouvoir le système des catégories, de traduire les lignes directrices en d'autres langues et de nous assurer qu'elles sont appliquées efficacement, afin d'optimiser le potentiel du système mondial d'aires protégées à perpétuité. 


\section{Remerciements}

La révision des lignes directrices de l'UICN est le résultat de discussions longues et complètes au sein de l'UICN. Nous sommes très reconnaissants envers les membres de l'UICN, de la Commission mondiale des aires protégées et du Groupe de travail sur les catégories qui nous ont aidés à préparer et à nous accorder sur le texte final. Notre publication fait suite à cette révision et elle a été rendue possible grâce à la généreuse contribution financière de la Fundación Biodiversidad espagnole. La Fundación Biodiversidad (Fondation Biodiversité) est une fondation sans but lucratif établie en 1998 suite aux engagements pris par l'Espagne après sa ratification de la Convention sur la diversité biologique. Elle réalise des activités dans le domaine de la conservation, de l'étude, et de l'utilisation durable de la biodiversité et aussi dans la coopération internationale pour le développement. Par cette coopération internationale, la Fundación Biodiversidad fait en sorte d'unir des efforts et de créer des synergies ainsi que d'encourager la coopération avec des organisations, des institutions et des programmes nationaux et internationaux.

Nous remercions tout d'abord les nombreuses personnes qui ont apporté leurs commentaires sur le projet Parlons la même langue. Le rapport final fut alors rédigé par Kevin Bishop, Nigel Dudley, Adrian Phillips et Sue Stolton, constituant ainsi la recherche historique qui a mené à la révision des catégories. Une liste complète de tous ceux que nous voulons remercier se trouve dans le rapport de ce projet et, depuis peu, nous pouvons aussi citer Natalia Danilina, vice-présidente de la CMAP pour l'Eurasie du Nord, qui a peaufiné la traduction de tout le rapport en russe.

Ensuite, nous exprimons nos remerciementsà tous ceux qui ont écrit des articles commandités ou indépendants sur l'application des catégories et ont fait des suggestions pour le nouveau texte. Parmi eux, citons : Robin Abell, José Antonio Atauri, Christian Barthod, Charles Besancon, Harry Biggs, Luigi Boitani, Grazia Borrini-Feyerabend, Peter Bridgewater, Jessica Brown, Phillip Bubb, Neil Burgess, José Courrau, Roger Crofts, Nick Davidson, Jon Day, Phillip Deardon, Benita Dillon, Charlie Falzon, Lucy Fish, Pete Frost, Roberto Gambino, John Gordon, Craig Groves, David Harmon, Marc Hockings, Sachin Kapila, Cyril Kormos, Ashish Kothari, Dan Laffoley, Harvey Locke, Stephanie Mansourian, Josep-Maria Mallarach, Claudio Maretti, Carole Martinez, Kenton Miller, Brent Mitchell, John Morrison, C. Niel, Gonzalo Oviedo, Jeffrey Parrish, Andrew Parsons, Marc Patry, Jean-Marie Petit, Adrian Phillips, Kent Redford, Liesbeth Renders, Carlo Rondinini, Deborah Bird Rose, Fausto Sarmiento, David Sheppard, Daniela Talamo, Daniel Vallauri, Bas Verschuuren, John Waugh et Bobby Wishitemi. BP a assuré le financement de la production de certains de ces articles et nous les remercions très chaleureusement pour leur appui.
Une étape critique du processus de révision fut la concrétisation du Sommet de l'UICN sur les Catégories qui s'est déroulé à Almeria, en Espagne, du 7 au 11 mai 2007. Le Sommet sur les Catégories fut organisé et réalisé avec le support financier et institutionnel de la Junta de Andalucia, de la Fundación Biodiversidad et du Centre UICN de Coopération pour la Méditerranée. Le Conseil régional pour l'Environnement de la Junta de Andalucia a fourni un soutien logistique et technique, sous la forme d'études de cas et d'activités de terrain au cours du Sommet, qui ont fortement contribué à sa réussite. Le Conseil régional pour l'Environnement de la Junta de Andalucia est l'agence qui, au sein du Gouvernement régional d'Andalousie, est responsable de la conservation de la nature, de l'application des réglementations et des politiques gouvernementales sur l'utilisation et la gestion des ressources naturelles, de la déclaration et de la gestion des aires protégées ainsi que de la définition, du développement et de la mise en œuvre de la stratégie et des politiques en matière de mitigation et d'adaptation concernant les changements climatiques.

Un grand nombre de personnes ont consacré une semaine à discuter de la révision des catégories pendant le Sommet UICN sur les Catégories. Nous devons un merci tout particulier à ces experts-ci qui y ont participé : Tarek Abulhawa, Andrés Alcantara, Germán Andrade, Alexandru Andrasanu, Suade Arancli, Margarita Astralaga, José Antonio Altauri, Jim Barborak, Brad Barr, Christian Barthod, Louis Bélanger, Charles Besancon, Ben Böer, Grazia Borrini-Feyerabend, Peter Bridgewater, Tom Brooks, Jessica Brown, Susana Calvo Roy, Sonia Castenãda, Carles Castell Puig, Miguel Castroviejo Bolivar, Peter Cochrane, Peter Coombes, José Courrau, Botella Coves, Roger Crofts, Marti Domènech I Montagut, Marc Dourojeanni, Holly Dublin, Nigel Dudley, Abdellah El Mastour, Ernest Enkerlin Hoeflicj, Reinaldo Estrada, Jordi FalgaronaBosch, Antonio Fernández de Tejada González, Georg Frank, Roberto Gambino, Javier Garat, Sarah Gindre, Craig Groves, José Romero Guirado, Manuel Francisco Gutiérrez, Heo HagYoung, Marc Hockings, Rolf Hogan, Bruce Jeffries, Vicente Jurado, Ali Kaka, Sachin Kapila, Seong-II Kim, Cyril Kormos, Meike Kretschmar, Zoltan Kun, Dan Laffoley, Kari Lahti, Maximo Liberman Cruz, Harvey Locke, Axel Loehken, Arturo Lopez, Elena López de Montenegro, Nik Lopoukhine, Ibanez Luque, Maher Mahjoub, Josep Maria Mallarach, Moses Mapesa, Claudio Maretti, Vance Martin, María Teresa Martín Crespo, Carole Martinez, Baldomero Martinez, Julia Marton-Lefevre, Mehrasa Pehrdadi, Rosa Mendoza Castellón, Kenton Miller, Susan Miller, Carmen Miranda, Fernando Molina, Sophie Moreau, Gérard Moulinas, Marta Múgica, Eduard Müller, Anread Müseler, Olav Nord-Varhaug, Juan Carlos Orella, Gonzalo Oviedo, Ana Pena, Milagros Pérez Villalba, Christine 
Pergent-Martini, Rosario Pintos Martin, Anabelle Plantilla, Francisco Quiros, Mohammed Rafiq, Tamica Rahming, Anitry Ny Aina Ratsifandrihamanana, Kent Redford, Manuel Rodriguez de Los Santos, Pedro Rosabal, Juan Carlos Rubio Garcia, Alberto Salas, Francisco Sanchez, Ana Elena Sánchez de Dios, José Luis Sánchez Morales, Mohammed Seghir Melouhi, Peter Shadie, David Sheppard, Sue Stolton, Gustavo Suárez de Freitas, Daniela Talamo, Tony Turner, Rauno Väisänen, Tafe Veselaj, Nestor Windevoxhel et Stephen Woodley.

Il y eut aussi des réunions régionales pour discuter des catégories lors de la 2ème Conférence de l'Heritage Parks Conference de l'ANASE et de la 4ème Conférence régionale sur les aires protégées en Asie du Sud-Est, à Sabah, en Malaisie ; en association avec le Centre mondial de surveillance de la conservation du PNUE, à Nairobi, Kenya ; au Second Congrès de parcs d'Amérique Latine à Bariloche, en Argentine et à la Réunion européenne de la CMAP à Barcelone, en Espagne. Notre reconnaissance va aux organisateurs, y compris Christi Nozawa, Anabelle Plantilla, Geoffrey Howard, Sue Stolton, Carmen Miranda et Roger Crofts. Nous remercions aussi tous ceux qui ont pris part aux ateliers et dont les idées ont contribué aux lignes directrices finales.

Des réunions eurent également lieu au Conseil international des mines et métaux et à l'International Petroleum Environmental Conservation Association, tous deux à Londres, ainsi que lors d'un rassemblement spécial de représentants d'industries et de membres de l'UICN à Gland, en Suisse ; nous remercions les organisateurs de ces rencontres.

De nombreuses personnes ont commenté la définition d'une aire protégée, l'ensemble des lignes directrices ou seulement une partie, et beaucoup d'autres encore ont participé au "e-débat ". Parmi ceux qui ont envoyé des commentaires écrits, qui ont pris part, voire organisé des rencontres se trouvent, en plus des personnes déjà citées ci-dessus : Mike Appleton, Alberto Arroyo, Andrea Athanus, Tim Badman, John Benson, Juan Bezaury, Stuart Blanch, Andrer Bouchard, José Briha, Kenneth Buk, Eduardo Carqueijeiro, Brian Child, Thomas Cobb, Nick Conner, Marina Cracco, Adrian Davey, Fekadu Desta, JeanPierre d'Huart, Paul Eagles, Joerg Elbers, Neil Ellis, Penny Figgis, Frauke Fisher, James Fitzsimmons, Gustavo Fonseca, Alistair Gammell, George Gann, Brian Gilligan, Fernando Ghersi, Hugh Govan, Mary Grealey, Michael Green, Larry Hamilton, Elery Hamilton Smith, Alan Hemmings, John Hough, Pierre Hunkeler, Glen Hvengaard, Tilman Jaeger, Jan Jenik, Graeme Kelleher, Richard Kenchington, Saskia de Koning, Linda Krueger, Barbara Lausche, Richard Leakey, Mary Kay LeFevour, Li Lifeng, Heather MacKay, Brendan Mackey, Dave MacKinnon, Vinod Mathur, Nigel Maxted, Jeffrey McNeely, Mariana Mesquita, Paul Mitchell, Russ Mittermeier,
Geoff Mosley, Fulori Nainoca, Juan Oltremari, Sarah Otterstrom, Thymio Papayanis, Jamie Pittock, Sarah Pizzey, Dave Pritchard, Allen Putney, Joanna Robertson, Jaime Rovira, Tove Maria Ryding, Heliodoro Sánchez, Andrej Sovinc, Rania Spyropoulou, Erica Stanciu, David Stroud, Surin Suksawan, Martin Taylor, Djafarou Tiomoko, Joseph Ronald Toussaint, Frank Vorhies, Daan Vreugdenhil, Haydn Washington, Sue Wells, Rob Wild, Graeme Worboys, Eugene Wystorbets et Edgard Yerena. Nombreux sont ceux qui ont envoyé des réponses collectives, reflétant les avis d'un certain nombre de collègues, d'institutions ou d'ONG.

David Sheppard, Pedro Rosabal, Kari Lahti, et Tim Badman du Programme UICN pour les aires protégées (PAP) ont apporté leur contribution technique et une orientation politique tout au long du processus ; Delwyn Dupuis, Anne Erb et Joanna Erfani (PAP) nous ont aussi fourni l'assistance et le support adminisratifs indispensables du Siège de l'UICN, à Gland. Nik Lopoukhine, président de la CMAP, a constamment soutenu le processus, tout comme les membres du Comité de direction de la CMAP. Citons particulièrement Trevor Sandwith, Roger Crofts et Marc Hockings qui tous ont examiné attentivement l'ensemble du texte, et Grazia Borrini-Feyerabend et Ashish Kothari qui ont commenté les nombreuses versions de la section sur la gouvernance. Les conseils techniques et politiques de Gonzalo Oviedo, conseiller principal de l'UICN en matière de politique sociale, furent essentiels pour toutes les questions de gouvernance et de populations autochtones.

Peter Cochrane et Sarah Pizzey de Parks Australia ont préparé et appuyé notre long périple dans cinq états australiens pour discuter des catégories avec des dizaines de professionnels des aires protégées, aussi bien dans des réunions que sur le terrain. Cette contribution a largement aidé à notre compréhension des défis et des opportunités en définissant les nouvelles lignes directrices et nous a permis de tester nos idées.

Le travail sur la catégorie Ib fut assuré par la Wilderness Task Force présidée par Vance Martin, le rôle principal pour les catégories étant assuré par Cyril Kormos. La position de l'UICN pour la catégorie $\mathrm{V}$ a été mieux définie grâce à deux réunions du groupe de travail spécial consacrées aux approches par paysages, généreusement financées par le Gouvernement catalan et par un consortium d'agences de conservation britanniques : Natural England, Scottish Natural Heritage et le Countryside Council for Wales. Jessica Brown préside le groupe de travail et a organisé les réunions avec l'aide, respectivement, de Jordi Falgarone et d'Andy Brown. La position concernant la catégorie VI a été définie grâce au travail d'un nouveau groupe de travail présidé par Claudio Maretti et lors d'une réunion dans le cadre du Congrès des Parcs d'Amérique Latine et des Caraibes de Bariloche, en Argentine. 


\section{Introduction}

Ces lignes directrices sont présentées pour aider à l'application des catégories d'aires protégées de l'UICN, qui classifient les aires protégées en fonction de leurs objectifs de gestion. Les catégories sont reconnues par les organes internationaux tels que les Nations unies et par de nombreux gouvernements nationaux comme étant la norme globale pour définir et enregistrer les aires protégées et, en tant que telles, elles sont de plus en plus souvent intégrées dans les législations nationales. Par exemple, le Programme de travail de la CDB sur les aires protégées " reconnaît l'intérêt d'un système de classification internationale unique des aires protégées et l'avantage de fournir des informations qui soient comparables entre les pays et les régions et, à cet égard, il se félicite donc des efforts de la Commission mondiale des aires protégées de l'UICN visant à affiner le système des catégories de l'UICN...".

Les lignes directrices apportent autant de clarté que possible en ce qui concerne la signification et l'application des catégories. Elles décrivent la définition et les catégories et discutent leur application dans des approches de gestion ou des biomes particuliers.

Lintention première du système de catégories de gestion des aires protégées de l'UICN était de créer une appréhension commune des aires protégées, à l'intérieur et entre les pays. Ceci est bien statué dans l'introduction des Lignes directrices par le président de ce qui était, à l'époque, la CPNAP (Commission des parcs nationaux et des aires protégées de l'UICN, connue aujourd'hui sous le nom de Commission mondiale des aires protégées), P.H.C. (Bing) Lucas qui écrivait : "Ces directives revêtent une importance toute particulière parce qu'elles s'adressent à tous ceux et celles que les aires protégées intéressent professionnellement... en proposant aux administrateurs de sites, aux planif-cateurs, aux chercheurs, aux politiciens et aux groupes de citoyens de tous les pays, un langage commun pour faciliter les échanges d'informations et d'idées" (UICN 1994).

Comme le remarque Phillips (2007), les Lignes directrices de 1994 visaient aussi à : « réduire la confusion quant à l'utilisation de nombreux termes différents pour décrire les aires protégées; fournir des normes internationales pour les comptes-rendus globaux ou régionaux et les comparaisons entre pays, en employant un cadre commun pour la collecte, la manipulation et la diffusion des données sur les aires protégées; et, en général, améliorer la communication et la compréhension entre tous ceux qui sont engagés dans la conservation".
Lutilisation de ces catégories d'aires protégées comme moyen de "parler la même langue " s'est largement répandue depuis l'adoption des lignes directrices en 1994. En particulier, il existe un grand nombre d'applications du système de catégories en politique, à tous les niveaux : international, régional et national. Les lignes directrices actuelles couvrent donc une plus vaste gamme de questions et donnent plus de détails que la version de 1994. Elles seront, si nécessaire, complétées par des orientations plus détaillées pour des catégories précises, une application à des biomes particuliers ou à des aires spécialisées. Suite à des consultations poussées avec l'UICN et ses membres, un certain nombre de changements supplémentaires se sont ajoutés depuis 1994, y compris à la définition d'une aire protégée et à certaines des catégories.

\section{Les termes « aires protégées » doivent-ils être inclusifs ou exclusifs ?}

Une question fondamentale liée à la définition et aux catégories d'aires protégées est de savoir si les mots « aire protégée » devraient être des termes généraux qui peuvent englober un très large éventail de types de gestion sur terre et en mer qui, incidemment, ont une certaine valeur en matière de conservation de la biodiversité et du paysage, ou s'ils devraient, au contraire, être des termes plus précis qui décrivent une forme particulière de système de gestion spécialement destinée à la conservation. L'interprétation differe selon les pays, ce qui rend parfois les comparaisons difficiles : certains des sites qui " comptent " comme aires protégées dans un pays ne seront pas nécessairement considérés comme tels dans un autre. L'UICN a tenté d'arriver à un certain consensus sur cette question parmi les parties prenantes clés. Tout en reconnaissant qu'il appartient aux pays eux-mêmes de déterminer ce qu'ils entendent par aire protégée, le poids de l'opinion parmi tous les membres de l'UICN, entre autres, semble peser en faveur d'un renforcement de la définition générale.

Une des implications sera que toutes les aires protégées qui sont importantes pour la conservation - par exemple, des forêts bien gérées, des zones d'utilisation durable, des zones d'entraînement militaire ou encore diverses formes de désignation de paysage au sens large - ne seront pas des " aires protégées " comme les reconnaît l'UICN. Il n'est pas dans nos intentions de minimiser ou d'entraver ce genre d'efforts de gestion durable. Nous reconnaissons au contraire que ces approches de la gestion sont importantes pour la conservation, mais elles se situent en dehors de ce que l'UICN définit comme aire protégée, ainsi que le décrivent ces lignes directrices. 


\section{Historique}

La première section des lignes directrices plante le décor en présentant ce que I'UICN entend par « aire protégée ». Elle reprend l'histoire des catégories d'aires protégées de I'UICN, y compris le processus actuel de révision des lignes directrices. Elle explique ensuite les principales raisons d'être des catégories comme les entend I'UICN. Enfin, un glossaire donne la définition des termes clés utilisés dans les lignes directrices, pour s'assurer que leur compréhension est cohérente. 


\section{Aires protégées}

Les aires protégées sont essentielles pour la conservation de la biodiversité. Elles sont les pierres angulaires de pratiquement toutes les stratégies nationales et internationales de conservation, elles sont mises en réserve pour conserver le bon fonctionnement des écosystèmes naturels, pour servir de refuges aux espèces et pour préserver les processus écologiques qui ne peuvent pas survivre dans les paysages terrestres ou marins gérés de façon plus intensive. Les aires protégées sont les jalons qui nous permettent de comprendre les interactions entre les hommes et le monde naturel. Aujourd'hui, elles sont souvent le seul espoir qui nous reste pour empêcher que de nombreuses espèces menacées ou endémiques disparaissent à jamais. Elles viennent compléter les mesures pour réaliser la conservation et l'utilisation durable de la diversité biologique en dehors des aires protégées, en accord avec les directives de la CDB telles que les Principes d'Addis Abeba et du Malawi (CDB VII/11-12). La plupart des aires protégées se trouvent dans des écosystèmes naturels ou quasinaturels, ou qui sont en voie de retrouver ce caractère, mais il existe des exceptions. Beaucoup recèlent des éléments majeurs de l'histoire ou de processus terrestres alors que d'autres documentent la subtile interaction entre l'activité humaine et la nature dans des paysages culturels. Des aires protégées plus vastes et plus naturelles procurent aussi un espace pour l'évolution et pour l'adaptation et la restauration écologiques futures, toutes deux de plus en plus importantes dans les conditions de rapides changements climatiques actuels.

De tels endroits représentent aussi pour les hommes des avantages directs. Les gens - aussi bien ceux qui vivent dans ou près des aires protégées que ceux qui en sont plus éloignés - profitent des possibilités de récréation et de ressourcement qu'offrent les parcs nationaux et les zones de nature sauvage, du potentiel génétique des espèces sauvages, et des services environnementaux fournis par les écosystèmes naturels, tel l'approvisionnement en eau. De nombreuses aires naturelles sont aussi essentielles pour les sociétés humaines vulnérables et préservent des endroits importants, comme des sites naturels sacrés. Bien que de nombreuses aires protégées soient instaurées par les gouvernements, de plus en plus sont désormais créées par des communautés locales, des populations autochtones, des organisations environnementales, des particuliers, des sociétés, etc.

Le monde naturel suscite un intérêt énorme et toujours croissant, et les aires protégées nous donnent la possibilité d'interagir avec la nature d'une manière qui est de plus en plus difficile à trouver ailleurs. Elles nous donnent un espace qui se fait rare sur une planète de plus en plus aménagée et surpeuplée.
Les aires protégées représentent aussi un engagement pour les générations futures. La plupart des gens croient aussi que nous avons l'obligation éthique d'empêcher la perte des espèces causée par nos actes, et ceci est conforté par les enseignements d'une large majorité des croyances religieuses mondiales (Dudley et al. 2006). La protection de paysages terrestres et marins emblématiques est également considérée comme importante d'une perspective culturelle plus large, et les aires protégées majeures sont aussi importantes pour le patrimoine d'un pays que, par exemple, des bâtiments célèbres comme la cathédrale Notre-Dame ou le Taj Mahal, des équipes nationales de football ou des œuvres d'art.

\section{Croissance dans le système mondial des aires protégées}

Aujourd'hui, environ un dixième de la surface terrestre bénéficie de l'une ou l'autre forme de protection. Au cours des quarante dernières années, l'ensemble mondial des aires protégées est passé de la taille du Royaume-Uni à celle de l'Amérique du Sud. Cependant de sérieux défis persistent. De nombreuses aires protégées ne sont pas encore complètement établies ou gérées. Les aires marines protégées sont à la traîne, loin derrière les aires protégées terrestres ou d'eau douce, même s'il y a actuellement des efforts considérables pour corriger cette situation. La grande majorité des aires protégées ont été identifiées et enregistrées au cours du vingtième siècle, dans ce qui fut presque certainement le plus vaste et le plus rapide changement conscient d'aménagement du territoire de toute l'histoire (même s'il ne fut pas aussi vaste que les dégradations largement non planifiées qui eurent lieu durant la même période). Ce glissement des valeurs doit encore être pleinement reconnu et compris. On continue à créer des aires protégées ; ce mouvement a même connu un essor particulier en 2004, lorsque la Convention sur la diversité biologique (CDB) a lancé un ambitieux Programme de travail sur les aires protégées, basé sur les principaux résultats $\mathrm{du} \mathrm{V}^{\mathrm{ème}}$ Congrès mondial sur les parcs, ${ }^{1}$ qui vise à compléter les systèmes d'aires protégées écologiquement représentatifs dans le monde entier et qui compte près d'une centaine de cibles à atteindre dans un délai fixé. Cela est vraiment nécessaire parce que, même si le taux de croissance fut impressionnant, de nombreuses aires protégées ont été créées dans des régions reculées, non ou peu peuplées, telles que des montagnes, des champs de glace et la toundra, et que les systèmes d'aires protégées révèlent encore de notables lacunes dans les écosystèmes forestiers ou de prairies, dans les déserts ou semi-déserts, dans les milieux d'eau douce et, particulièrement, dans les régions marines et côtières. Dans le monde, de nombreuses espèces végétales et animales n'ont pas de population viable dans les aires protégées, et une proportion considérable d'entre elles 
vivent totalement en dehors des aires protégées (Rodrigues $e t$ al. 2004). Il est donc probable que l'on continuera à créer de nouvelles aires protégées à l'avenir. Un important développement de la dernière décennie fut le professionnalisme accru dans la sélection des aires protégées, en recourant notamment à des techniques telles que l'analyse des lacunes écologiques (Dudley et Parrish 2006).

Pendant cette même période, nous avons aussi de mieux en mieux compris comment ces aires devraient être gérées. Dans le rush pour établir des aires protégées, souvent parce qu'il fallait sauver d'un soudain assaut de développement certaines portions naturelles terrestres ou aquatiques, des aires protégées furent mises en réserve sans que l'on fasse une analyse sérieuse des compétences et des capacités qu'il allait falloir pour les préserver. Les connaissances s'améliorent rapidement à tous les niveaux de gestion, des planificateurs jusqu'aux gardes sur le terrain, et il y a un réseau de plus en plus sophistiqué de bénévoles prêts à soutenir le développement des systèmes d'aires protégées. Dans une évolution parallèle, de nombreuses communautés locales et des populations traditionnelles et autochtones commencent à voir les aires protégées comme un moyen de protéger des lieux qui sont importants pour elles, par exemple des sites naturels sacrés ou des aires gérées pour certains avantages environnementaux tels que de l'eau pure ou le maintien des stocks de poissons.

\section{La diversité de la protection}

Les termes « aire protégée » sont donc un peu courts pour englober une gamme parfois déconcertante d'appellations sur terre et en mer, dont certaines des plus connues sont parc national, réserve naturelle, zone de nature sauvage, aire de gestion des habitats ou des espèces et paysages terrestres ou marins protégés, mais qui peuvent aussi inclure des approches telles que les zones de conservation communautaire. Plus important, ces termes embrassent aussi une vaste gamme d'approches de gestion différentes, allant des sites strictement protégés où peu, voire aucune personne n'est autorisée à pénétrer aux approches beaucoup moins restrictives où la conservation est intégrée dans le mode de vie traditionnel (et parfois pas si traditionnel que ça) et cohabite même avec l'extraction durable de ressources naturelles, en passant par des parcs où l'accent est mis sur la conservation mais où les visiteurs sont néanmoins les bienvenus. Certaines aires protégées interdisent toute activité telle que la collecte de nourriture, la chasse ou l'extraction de ressources naturelles alors que dans d'autres, ces activités sont une composante tolérée, voire indispensable de la gestion. Les approches adoptées dans les aires protégées terrestres, d'eau douce, ou marines peuvent aussi différer significativement, et ces différences sont expliquées plus loin dans des lignes directrices.

Cette variété reflète le fait que la conservation ne se réalise pas de la même façon dans chaque situation et que ce qui peut être souhaitable et faisable à un endroit peut s'avérer contreproductif ou politiquement impossible ailleurs. Les aires protégées sont le résultat d'un accent bienvenu mis sur la réflexion à long terme et sur l'attention au monde naturel ; cependant, elles impliquent parfois un prix à payer par ceux qui vivent dans ou à proximité des aires qui sont protégées, en termes de perte de droits, de terres ou d'accès aux ressources. Il y a des pressions croissantes, très justifiées, pour que l'on tienne correctement compte des besoins humains lorsque l'on crée des aires protégées, et ceux-ci doivent parfois faire l'objet de compromis quant aux besoins de la conservation. Alors que dans le passé, les gouvernements prenaient des décisions au sujet des aires protégées et en informaient la population par la suite, aujourd'hui on insiste davantage sur de larges consultations avec les parties prenantes et sur des décisions conjointes quant à la façon dont il faudrait mettre en réserve et gérer ces terres. De telles négociations ne sont jamais faciles mais elles produisent d'habitude des résultats plus solides et plus durables, tant pour la conservation que pour les gens.

L'UICN reconnaît que de nombreuses approches pour créer et gérer des aires protégées sont valides et peuvent faire des contributions substantielles à des stratégies de conservation. Cela ne signifie pas qu'elles sont toutes aussi intéressantes dans chaque situation ; la capacité de choisir et de combiner différentes approches de gestion au sein d'une aire protégée et entre aires protégées est souvent essentielle pour mettre au point un système d'aires protégées qui fonctionne efficacement. Certaines situations requièrent une protection stricte ; d'autres peuvent fonctionner, voire fonctionnent mieux avec des approches de la gestion moins restrictives ou avec un zonage de différentes stratégies de gestion dans la même aire protégée.

\section{Décrire différentes approches}

Pour essayer de rendre compte et de décrire les différentes approches, l'UICN a convenu d'une définition de ce qu'est et de ce que n'est pas un aire protégée et elle a ensuite identifié six catégories différentes d'aires protégées basées sur les objectifs de gestion, l'une d'entre elles étant subdivisée en deux parties. Bien que les catégories aient été, au départ, destinées modestement à aider à récolter des données et des informations sur les aires protégées, elles sont devenues avec le temps un outil plus complexe. Aujourd'hui, les catégories concrétisent la philosophie de l'UICN en matière d'aires protégées, mais elles aident aussi à fournir un cadre où les différentes stratégies de protection peuvent se combiner entre elles, avec le support de systèmes de gestion en dehors des aires protégées, en une approche cohérente pour conserver la nature. Les catégories de l'UICN sont désormais utilisées dans des objectifs aussi divers que la planification et l'instauration de réglementations, ou la négociation des utilisations des sols et de l'eau. Ce livre décrit les catégories et explique comment elles peuvent servir à planifier, mettre en ouvre et évaluer les stratégies de conservation. 
Un mot d'avertissement : les aires protégées existent sous des formes incroyablement diverses-en taille, localisation, approches et objectifs de gestion. Toute tentative de comprimer une collection aussi riche et compliquée en une demi-douzaine de petites cases bien nettes ne pourrait être au mieux qu'approximative. La définition et les catégories d'aires protégées de l'UICN ne sont pas une camisole de force mais un cadre pour orienter une meilleure application des catégories.

\section{Historique des catégories d'aires protégées de I'UICN}

Alors que des aires protégées, dans le sens moderne du terme, étaient créées dans un pays après l'autre tout au long du $20^{\text {ème }}$ siècle, chaque nation a développé son approche pour les gérer, et il n'existait au départ ni normes, ni terminologie communes. Il en résulte que de nombreux termes différents sont utilisés au niveau national pour décrire les aires protégées et qu'il y a aussi toute une variété de systèmes internationaux d'aires protégées qui ont été créés dans le cadre de conventions mondiales (ex. les Sites du patrimoine mondial) et d'accords régionaux (ex. les sites Natura 2000 en Europe).

Le premier effort pour clarifier la terminologie fut fait en 1933, à la Conférence internationale pour la protection de la faune et de la flore, à Londres. Celle-ci institua quatre catégories d'aires protégées : parc national, réserve naturelle intégrale, réserve de faune et de flore et réserve avec interdiction de chasse et de collecte. En 1942, la Convention de Londres sur la protection de la nature dans l'hémisphère occidental a aussi intégré quatre types : parc national, réserve nationale, monument naturel et réserve naturelle intégrale (Holdgate 1999).

En 1962, la toute nouvelle Commission des parcs nationaux et des aires protégées (CPNAP) de l'UICN, aujourd'hui Commission mondiale des aires protégées (CMAP), a préparé une Liste mondiale des parcs nationaux et des réserves équivalentes pour la Première Conférence mondiale sur les parcs nationaux à Seattle, avec un article de C. Frank Brockman (1962) sur la nomenclature. En 1966, l'UICN a produit une seconde version de ce qui est devenu une publication régulière connue aujourd'hui sous le nom de Liste des Nations unies des parcs nationaux et des aires protégées, en utilisant un système de classification simple : parcs nationaux, réserves scientifiques et monuments naturels. La deuxième Conférence mondiale des parcs de 1972 a demandé que l'UICN "définisse les différentes raisons pour lesquelles des aires protégées sont mises en réserve et de mettre au point des normes et une nomenclature adaptées à de telles aires " (Elliott 1974).

C'est tout cela qui a conduit à la décision de la CPNAP de développer un système de catégories pour les aires protégées. Le rapport d'un groupe de travail (UICN 1978) arguait qu'un système de catégorisation devrait montrer comment les parcs nationaux pourraient être complétés par d'autres types d'aires protégées, aider les nations à développer des catégories de gestion qui reflètent leurs besoins propres, aider l'UICN à rassembler et à analyser les données sur les aires protégées, supprimer les ambigüités et les incohérences, et garantir que "quelle que soit la nomenclature utilisée par les nations..., une aire de conservation puisse être reconnue et classée en fonction des objectifs pour lesquels elle est réellement gérée ". Dix catégories furent proposées, définies principalement par leur objectif de gestion, chacun étant considéré comme important et aucune catégorie n'étant, en soi, plus valable qu'une autre :

Groupe A : Catégories pour lesquelles la CPNAP assurera une responsabilité particulière

I Réserve scientifique / Réserve naturelle intégrale

II Parc national

III Monument naturel / Elément naturel marquant

IV Réserve de conservation de la nature / Réserve naturelle dirigée / Sanctuaire de faune

V Paysage terrestre protégé

Groupe B : Autres catégories importantes pour l'UICN, mais qui ne sont pas exclusivement dans le rayon d'action de la CPNAP

VI Réserve de ressources naturelles

VII Région biologique naturelle / Réserve anthropologique

VIII Région naturelle aménagée à des fins d'utilisation multiple / Zone de gestion des ressources naturelles

Groupe C : Catégories qui font partie de programmes internationaux

IX Réserve de la biosphère

$\mathrm{X} \quad$ Bien du patrimoine mondial (naturel)

Cependant, le système a vite révélé ses limites. Il ne contenait aucune définition d'une aire protégée, plusieurs termes servaient à décrire toute la gamme des dix catégories, une même aire protégée pouvait se trouver dans plus d'une catégorie, et le système n'abordait pas le domaine marin.

\section{Révision et propositions de nouvelles catégories}

En 1984, la CPNAP a créé un groupe d'étude pour mettre à jour les catégories. Il a remis son rapport en 1990, en proposant qu'un nouveau système soit élaboré à partir des catégories I à V de 1978 et qu'il abandonne les catégories VI à X (Eidsvik 1990). Ce rapport a été transmis en 1992 au Congrès mondial des parcs de Caracas, au Venezuela. Un atelier de trois jours a proposé de conserver une catégorie qui serait proche de celle qui avait été auparavant la catégorie VIII pour les aires protégées où l'utilisation durable des ressources naturelles était un des objectifs. Le Congrès a soutenu cette proposition et, en janvier 1994, la réunion de l'Assemblée générale de l'UICN à Buenos Aires a approuvé le nouveau système. Ces lignes directrices furent publiées par l'UICN et par le Centre 
mondial de surveillance de la conservation (World Conservation Monitoring Centre (WCMC)) plus tard dans l'année (UICN 1994). Elles donnent une définition de l'aire protégée - Une portion de terre etlou de mer vouée spécialement à la protection et au maintien de la diversité biologique, ainsi que des ressources naturelles et culturelles associées, et gérée par des moyens efficaces, juridiques ou autres - et des six catégories :

\section{Aires gérées principalement pour : \\ I Protection intégrale [Ia) Réserve naturelle intégrale et Ib) Zone de nature sauvage] \\ II Conservation de l'écosystème et protection (p.ex. Parc national) \\ III Conservation d'éléments naturels (p.ex. Monument naturel) \\ IV Conservation par une gestion active (p.ex. Aire de gestion des habitats / espèces) \\ V Conservation d'un paysage terrestre / marin et loisirs (p.ex. Paysage terrestre / marin protégé) \\ VI Utilisation durable des écosystèmes naturels (p.ex. Aire protégée de ressources naturelles gérée)}

Les lignes directrices de 1994 se fondent sur des principes clés : le choix de la catégorie repose sur l'objectif premier de la gestion ; le placement dans une catégorie n'est pas un commentaire sur l'efficacité de la gestion; le système des catégories est international ; les noms nationaux des aires protégées peuvent varier ; toutes les catégories sont importantes ; ...mais elles impliquent une gradation de l'intervention humaine.

\section{Evolution depuis 1994}

Depuis la publication de ces lignes directrices, l'UICN encourage activement la bonne compréhension et l'utilisation du système de catégories. Elle s'est impliquée dans des publications sur la façon d'appliquer les lignes directrices dans des contextes spécifiques, géographiques ou autres (p.ex. EUROPARC et UICN 1999 ; Bridgewater et al. 1996) et dans un volume spécifique de lignes directrices pour la catégorie $\mathrm{V}$ d'aires protégées (Phillips 2002). Le système de catégories fut la pierre angulaire d'une prise de position de la CMAP sur l'exploitation minière et les aires protégées, exprimée dans une recommandation $\left(\mathrm{n}^{\circ} 2.82\right)$ adoptée par le Congrès mondial de la conservation à Amman en 2000.

L'UICN s'est assurée de l'adoption du système par la Convention sur la diversité biologique lors de la $7^{\text {’̀me }}$ Conférence des Parties à la CDB à Kuala Lumpur en février 2004. Lors du Congrès mondial des parcs, à Durban (2003) et du Congrès mondial de la conservation à Bangkok (2004), il y eut des propositions visant à ajouter une dimension de gouvernance aux catégories.

Enfin, l'UICN a soutenu un projet de recherche del'Université de Cardiff (GB) sur l'utilisation et les performances du système de 1994 : Parlons la même langue. Les résultats furent discutés une première fois au Congrès mondial des parcs en 2003 et ils furent publiés pour le Congrès mondial de la conservation en 2004 (Bishop et al. 2004). Un résumé des articles fut aussi publié dans PARKS en 2004 (UICN 2004). Ce projet a aidé à la création du Groupe de travail de la CMAP sur les catégories et à lancer le processus de révision qui a abouti à cette nouvelle série de lignes directrices.

\section{Le processus de révision actuel}

Ces lignes directrices sont le résultat d'un intense processus de consultation et de révision coordonné par un groupe de travail spécialement appointé par la CMAP, travaillant en étroite collaboration avec des membres de la CMAP et aussi avec les cinq autres commissions de l'UICN. Le groupe de travail a décidé de son plan de travail initial suite aux résultats du projet Parlons la même langue, mais avec un mandat plus large de l'UICN pour examiner tous les aspects des catégories. Il a passé 18 mois à récolter des informations, à parler et écouter au cours d'une succession d'étapes :

- Recherche : de nombreuses personnes appartenant ou non au réseau de la CMAP ont contribué à la révision de ces lignes directrices en rédigeant une série de documents de travail et en examinant divers aspects des catégories. Près de 40 articles furent écrits, allant de documents de discussion et d'interpellations diverses à des articles qui faisaient des propositions très spécifiques ou suggéraient des textes pour les nouvelles lignes directrices. Ensemble, ils constituent une ressource importante qui examine comment une gamme d'objectifs de gestion d'aires protégées contribue à leur conservation.

- Réunions et discussions : le groupe de travail a mené une série de réunions dans le monde entier, ou a contribué à des réunions, pour donner aux gens l'occasion d'exprimer leur avis, leurs espoirs et leurs inquiétudes au sujet des approches de gestion d'aires protégées. Les réunions clés comprenaient :

- Catégorie V : réunion conjointe avec le groupe de travail de la CMAP sur les paysages en Catalogne, Espagne, en 2006, avec le soutien du Gouvernement catalan, pour mettre au point une position sur la catégorie $\mathrm{V}$ et les approches par paysages, suivie par une autre réunion du groupe de travail dans le Nord Yorkshire, Angleterre, en 2008 ;

- Catégorie VI : réunion au Brésil pour préparer un document d'orientation et planifier un manuel technique en 2007 ;

- Europe : discussion lors de la réunion européenne de la CMAP à Barcelone pour rassembler les avis des membres de la CMAP en 2007 ;

- Afrique australe et de l'Est : atelier de deux jours à Nairobi en 2006 en collaboration avec le WCMC du PNUE, auquel ont participé des représentants de 13 états africains ; 
- Asie du Sud-est : atelier de deux jours sur la gouvernance et les catégories au cours d'une conférence régionale à Kota Kinabalu, à Sabah, en Malaisie, en 2007, avec des représentants de 17 pays ;

- Amérique latine : discussions au cours du congrès d'Amérique latine sur les aires protégées à Bariloche, en Argentine, en 2007, s'intéressant particulièrement aux questions portant sur la catégorie VI, les aires marines protégées et les réserves autochtones;

- Conseil international des mines et métaux : présentation suivie par une discussion qui aboutit à un document de travail des membres du CIMM en 2007.

- Il y eut aussi une série de réunions plus restreintes, par exemple avec le Comité britannique de l'UICN, le Conseil canadien des aires écologiques, le Programme du WWF pour la science de la conservation, Conservation International, l'UNESCO, des acteurs de l'industrie au siège de l'UICN, etc.

- De plus, il y eut un " sommet " global sur les catégories d'aires protégées en Espagne en mai 2007, financé et supporté techniquement par le Gouvernement régional andalou, le Ministère espagnol de l'Environnement et la Fundación Biodiversidad. Y ont assisté plus d'une centaine d'experts venus du monde entier, qui ont eu quatre jours pour discuter d'un large éventail de sujets liés aux catégories. Même si ce n'était pas un conseil censé prendre des décisions, les diverses positions de consensus développées durant la réunion ont aidé à la mise en forme des lignes directrices révisées.

- Site internet : Le groupe de travail a un portail sur le site internet de la CMAP, avec tous les articles intéressants, etc. disponibles (en anglais) sur : www.iucn.org/themes/ wcpa/theme/categories/about.html

- E-forum : dans la période préparatoire au sommet, l'UICN et le groupe de travail ont coordonné une e-discussion ouverte à tous au sujet des catégories; elle a fourni des informations inestimables pour la réflexion concernant les prochaines étapes du processus de révision.

Le projet de lignes directrices a été préparé pour la réunion du Comité de direction de la Commission mondiale des aires protégées en septembre 2007 ; il fut revu en fonction des commentaires des membres du Comité de direction. Les divers projets ne furent produits qu'en anglais, une restriction due à un manque de fonds, mais la version finale est produite intégralement en anglais, en français et en espagnol, avec des résumés dans d'autres langues. Les lignes directrices furent mises à la disposition de tous les membres de la CMAP et de toute autre partie intéressée pour commentaires, et de nombreux commentaires furent reçus et intégrés dans le texte.
Il y eut aussi une consultation distincte concernant la définition des aires protégées.

Le Comité de direction de la CMAP s'est à nouveau réuni en avril 2008 au Cap et il a discuté le projet en détail en session ouverte et en groupes distincts pour traiter les problèmes particuliers. Les décisions finales quant à ce qu'il fallait proposer aux membres de l'UICN furent prises, lorsque c'était nécessaire, par le président de la CMAP.

\section{Raison d'être des catégories de gestion des aires protégées de I'UICN}

LUICN considère les catégories de gestion des aires protégées comme une norme importante pour la planification, la création et la gestion des aires protégées ; cette section souligne les principales utilisations reconnues. Celles-ci se sont multipliées depuis les premières lignes directrices sur les catégories publiées en 1994, et la liste des utilisations possibles s'est allongée. D'autre part, les catégories servent parfois d'outils dans des cas qui n'avaient pas été envisagés, peut-être en raison de l'absence d'alternative, et nous devons distinguer les utilisations que l'UICN encourage de celles auxquelles elle s'oppose ou pour lesquelles elle veut rester neutre.

\section{Raisons d'être que I'UICN appuie et encourage activement}

Faciliter la planification des aires protégées et des systèmes d'aires protégées

- Fournir un outil pour planifier les systèmes d'aires protégées et les exercices plus vastes de planification de la conservation à l'échelle biorégionale ou écorégionale ;

- Encourager les gouvernements et les autres propriétaires ou gestionnaires d'aires protégées à mettre au point des systèmes d'aires protégées qui ont un éventail d'objectifs de gestion taillés à la mesure des conditions nationales et locales ;

- Reconnaître les différentes dispositions en matière de gestion et les divers types de gouvernance.

\section{Améliorer la gestion des informations concernant les aires protégées}

- Fournir des normes internationales pour aider à la collecte des données globales ou régionales et aux comptes-rendus sur les efforts de la conservation, faciliter les comparaisons entre pays et instaurer un cadre pour les évaluations globales et régionales;

- Fournir un cadre pour la collecte, la manipulation et la diffusion des données sur les aires protégées ;

- Améliorer la communication et la compréhension entre tous ceux qui sont engagés dans la conservation ; 
- Réduire la confusion qui est née de l'adoption de nombreux termes différents pour décrire le même genre d'aires protégées dans différentes parties du monde.

\section{Aider à réglementer les activités dans les aires protégées}

- Utiliser les catégories comme lignes directrices au niveau national et international pour aider à réglementer les activités, p.ex. en prescrivant certaines activités dans certaines catégories en fonction des objectifs de gestion de l'aire protégée.

\section{Raisons d'être qui deviennent de plus en plus communes, que I'UICN appuie et au sujet desquelles elle est prête à donner des conseils}

- Fournir une base pour la législation - un nombre croissant de pays utilisent les catégories de l'UICN comme une ou la base du classement de leurs aires protégées selon la loi ;

- Fixer les budgets - certains pays fondent l'échelle des budgets de leurs aires protégées sur leur catégorie ;
- Utiliser les catégories comme un argument de plaidoyer-des ONG utilisent les catégories comme un outil de campagne pour promouvoir des objectifs de conservation et des niveaux appropriés d'utilisation humaine ;

- Interpréter ou clarifier le droit foncier et la gouvernance certaines communautés autochtones et locales utilisent les catégories comme un outil pour les aider à établir des systèmes de gestion comme ceux des réserves autochtones ;

- Fournir des outils pour aider à planifier des systèmes d'aires protégées avec une gamme d'objectifs de gestion et de types de gouvernance.

\section{Raisons d'être auxquelles l'UICN s'oppose}

- Utiliser les catégories comme une excuse pour expulser des gens de leurs terres ancestrales;

- Changer de catégorie pour baisser le niveau de protection de l'environnement ;

- Utiliser les catégories pour argumenter en faveur d'un développement irréfléchi d'un point de vue environnemental dans les aires protégées. 



\section{Définitions et catégories}

Cette section souligne et explique la définition d'une aire protégée, d'un système d'aires protégées et des six catégories par I'UICN. La définition est clarifiée phrase par phrase et devrait être appliquée avec quelques principes connexes. Les catégories sont décrites en fonction de leur objectif majeur, d'autres objectifs, de caractéristiques distinctives, de leur rôle dans le paysage terrestre ou marin, d'aspects uniques et d'activités qui sont compatibles, ou non. 


\section{La nouvelle définition UICN d'une aire protégée}

La définition de I'UICN est donnée et expliquée, phrase par phrase

Des membres de l'UICN ont travaillé ensemble pour produire une nouvelle définition d'une aire protégée qui est donnée ci-dessous. La première version de cette nouvelle définition fut préparée au cours d'une réunion sur les catégories à Almeria, en Espagne, en mai 2007, et depuis lors, elle fut successivement affinée et révisée par de nombreuses personnes au sein de la CMAP/UICN.
Une aire protégée est : « Un espace géographique clairement défini, reconnu, consacré et géré, par tout moyen efficace, juridique ou autre, afin d'assurer à long terme la conservation de la nature ainsi que les services écosystémiques et les valeurs culturelles qui lui sont associés".

En appliquant le système des catégories, la première étape consiste à déterminer si le site répond à cette définition et la seconde à décider quelle est la catégorie la plus appropriée.

Cette définition contient beaucoup de choses en une courte phrase. Le Tableau 1 examine chaque mot et/ou phrase et développe sa signification.

Tableau 1. Explication de la définition d'une aire protégée

\begin{tabular}{|c|c|c|}
\hline Phrase & Explication & Exemples et nouveaux détails \\
\hline $\begin{array}{l}\text { Espace } \\
\text { géographique } \\
\text { clairement } \\
\text { défini }\end{array}$ & $\begin{array}{l}\text { Inclut les aires terrestres, marines et côtières, d'eau } \\
\text { douce ou une combinaison de deux ou plusieurs } \\
\text { d'entre elles. L' « espace » a trois dimensions, p. } \\
\text { ex. lorsque l'espace aérien au-dessus d'une aire } \\
\text { protégée est protégé contre le vol en rase-motte } \\
\text { ou, dans des aires marines protégées, quand une } \\
\text { certaine profondeur d'eau ou le fond de la mer } \\
\text { sont protégés mais que l'eau au-dessus ne l'est } \\
\text { pas : par contre, les zones situées sous la surface } \\
\text { ne sont parfois pas protégées (p. ex. ouvertes } \\
\text { à l'exploitation minière). "Clairement défini » } \\
\text { implique une aire définie dans l'espace avec des } \\
\text { limites reconnues et marquées. Ces limites peuvent } \\
\text { parfois être définies par des caractéristiques } \\
\text { physiques qui se déplacent avec le temps (p. ex. } \\
\text { berges de rivières) ou par des activités de gestion } \\
\text { (p. ex. zones de non-prélèvement convenues). }\end{array}$ & $\begin{array}{l}\text { La Réserve Naturelle de Wolong en } \\
\text { Chine (catégorie la, terrestre); le Parc } \\
\text { National du Lac Malawi, au Malawi } \\
\text { (catégorie II, principalement eau douce); } \\
\text { la Réserve Marine des Baies de } \\
\text { Masinloc et d'Oyon aux Philippines } \\
\text { (catégorie la, principalement marine) } \\
\text { sont des exemples d'aires situées dans } \\
\text { des biomes très différents mais qui sont } \\
\text { toutes des aires protégées. }\end{array}$ \\
\hline reconnu & $\begin{array}{l}\text { Implique que la protection peut inclure toute une } \\
\text { gamme de types de gouvernance déclarés par } \\
\text { la population ainsi que ceux identifiés par l'état, } \\
\text { mais que de tels sites doivent être reconnus d'une } \\
\text { certaine façon (en particulier en étant repris sur la } \\
\text { liste de la Base de données mondiale sur les aires } \\
\text { protégées - BDMAP). }\end{array}$ & $\begin{array}{l}\text { L'Anindilyakwa Indigenous Protected } \\
\text { Area (IPA) fut auto-déclarée par } \\
\text { les communautés aborigènes de la } \\
\text { Péninsule de Groote Eylandt ; c'est une } \\
\text { des nombreuses aires du patrimoine } \\
\text { communautaire (IPA) reconnues par le } \\
\text { gouvernement. }\end{array}$ \\
\hline consacré & $\begin{array}{l}\text { Implique un certain engagement contraignant envers } \\
\text { la conservation à long terme passant par, p.ex. : } \\
\text { - Conventions et accords internationaux } \\
\text { - Loi nationale, provinciale et locale } \\
\text { - Droit coutumier } \\
\text { - Engagements des ONG } \\
\text { - Fiducies privées et politiques des sociétés } \\
\text { - Programmes de certification. }\end{array}$ & $\begin{array}{l}\text { Cradle Mountain - Lake St Clair } \\
\text { National Park en Tasmanie, Australie } \\
\text { (catégorie II, état); Nabanka Fish } \\
\text { Sanctuary, Philippines (aire du } \\
\text { patrimoine communautaire); Port } \\
\text { Susan Bay Preserve, Washington, USA } \\
\text { (privée) sont tous des aires protégées } \\
\text { mais leur structure juridique varie } \\
\text { considérablement. }\end{array}$ \\
\hline géré & $\begin{array}{l}\text { Suppose quelques démarches actives pour } \\
\text { conserver les valeurs naturelles (et éventuellement } \\
\text { d'autres) pour lesquelles l'aire protégée fut créée ; } \\
\text { notons que « géré » peut inclure la décision de } \\
\text { laisser l'aire intacte si c'est la meilleure stratégie de } \\
\text { conservation. }\end{array}$ & $\begin{array}{l}\text { De nombreuses options sont possibles. Par } \\
\text { exemple, le Kaziranga National Park, en } \\
\text { Inde (catégorie II) est géré principalement } \\
\text { en contrôlant le braconnage et en retirant } \\
\text { les espèces invasives ; les îles de } \\
\text { l'Archipelago National Park en Finlande } \\
\text { sont gérées en employant des méthodes } \\
\text { agricoles traditionnelles pour préserver les } \\
\text { espèces associées aux prairies. }\end{array}$ \\
\hline
\end{tabular}


Tableau 1. Explication de la définition d'une aire protégée (suite)

\begin{tabular}{|c|c|c|}
\hline Phrase & Explication & Exemples et nouveaux détails \\
\hline $\begin{array}{l}\text { par tout moyen } \\
\text { efficace, } \\
\text { juridique ou } \\
\text { autre }\end{array}$ & $\begin{array}{l}\text { Signifie que les aires protégées doivent être } \\
\text { soit enregistrées, c.-à-d. reconnues dans le } \\
\text { cadre de la loi civile statutaire), soit reconnues } \\
\text { par une convention ou un accord international, } \\
\text { ou encore gérées par d'autres moyens } \\
\text { efficaces mais non enregistrés, comme les } \\
\text { réglementations traditionnelles reconnues selon } \\
\text { lesquelles fonctionnent les aires du patrimoine } \\
\text { communautaire ou les politiques d'organisations } \\
\text { non gouvernementales confirmées. }\end{array}$ & $\begin{array}{l}\text { Le Flinders Range National Park, en } \\
\text { Australie, est géré par l'autorité de l'Etat } \\
\text { de l'Australie du Sud ; l'Attenborough } \\
\text { Nature Reserve en GB est gérée par } \\
\text { le Nottinghamshire Wildlife Trust en } \\
\text { association avec la société productrice } \\
\text { de gravier qui possède le site ; et l'Alto } \\
\text { Fragua Indiwasi National Park de } \\
\text { Colombie est géré par les populations } \\
\text { ingano. }\end{array}$ \\
\hline afin d'assurer & $\begin{array}{l}\text { Implique une certaine efficacité. C'est un nouvel } \\
\text { élément qui n'était pas présent dans la définition de } \\
1994 \text { mais qui a été fermement requis, entre autres, } \\
\text { par de nombreux gestionnaires d'aires protégées. } \\
\text { Bien que la catégorie soit toujours déterminée par les } \\
\text { objectifs, l'efficacité de gestion sera progressivement } \\
\text { notée dans la Base de données mondiale sur les } \\
\text { aires protégées et, avec le temps, elle deviendra un } \\
\text { critère important qui contribuera à l'identification et à } \\
\text { la reconnaissance des aires protégées. }\end{array}$ & $\begin{array}{l}\text { La Convention sur la diversité } \\
\text { biologique demande aux Parties de } \\
\text { procéder à des évaluations de l'efficacité } \\
\text { de la gestion. }\end{array}$ \\
\hline à long terme & $\begin{array}{l}\text { Les aires protégées doivent être gérées dans la } \\
\text { durée et non comme une stratégie de gestion } \\
\text { temporaire }\end{array}$ & $\begin{array}{l}\text { Des mesures temporaires, comme les } \\
\text { mises en jachères de courte durée } \\
\text { bénéficiant de financements, les rotations } \\
\text { dans la gestion de forêts d'exploitation } \\
\text { ou des zones de pêche mises sous } \\
\text { protection temporaire ne sont pas des } \\
\text { aires protégées telles que les reconnaît } \\
\text { l'UICN. }\end{array}$ \\
\hline la conservation & $\begin{array}{l}\text { Dans le contexte de cette définition, la } \\
\text { conservation fait référence au maintien in-situ } \\
\text { d'écosystèmes et d'habitats naturels et semi- } \\
\text { naturels et de populations viables d'espèces } \\
\text { dans leurs environnements naturels et, dans le } \\
\text { cas d'espèces domestiquées ou cultivées (voir } \\
\text { la définition de l'agrobiodiversité dans l'Annexe), } \\
\text { dans l'environnement où elles ont développé leurs } \\
\text { propriétés distinctives. }\end{array}$ & $\begin{array}{l}\text { Le Parc National de Yellowstone } \\
\text { aux USA (catégorie II) a des buts de } \\
\text { conservation centrés particulièrement sur } \\
\text { le maintien de populations viables d'ours } \\
\text { et de loups, mais avec l'objectif plus large } \\
\text { de préserver le bon fonctionnement de } \\
\text { tout l'écosystème. }\end{array}$ \\
\hline de la nature & $\begin{array}{l}\text { Dans ce contexte, la nature fait toujours référence } \\
\text { à la biodiversité aux niveaux génétique, de } \\
\text { l'espèce et de l'écosystème et aussi, souvent, } \\
\text { à la géodiversité, le modelé, et d'autres valeurs } \\
\text { naturelles plus générales. }\end{array}$ & $\begin{array}{l}\text { Le Bwindi Impenetrable Forest } \\
\text { National Park en Ouganda (catégorie } \\
\text { II) est tout d'abord géré pour protéger } \\
\text { les forêts de montagne naturelles et } \\
\text { particulièrement les gorilles de montagne. } \\
\text { L'Island of Rum National Nature } \\
\text { Reserve, en Ecosse, (catégorie IV) fut } \\
\text { créée pour protéger ses caractéristiques } \\
\text { géologiques uniques. }\end{array}$ \\
\hline $\begin{array}{l}\text { et les services } \\
\text { écosystémiques } \\
\text { qui lui sont } \\
\text { associés }\end{array}$ & $\begin{array}{l}\text { Signifie ici les services de l'écosystème qui sont } \\
\text { liés mais n'interfèrent pas avec les objectifs de la } \\
\text { conservation de la nature. Ils peuvent comprendre } \\
\text { des services d'approvisionnement comme l'eau et } \\
\text { la nourriture; des services de régulation comme } \\
\text { celles des inondations, des sécheresses, de la } \\
\text { dégradation des sols et des maladies; des services } \\
\text { de soutien comme la formation des sols et le cycle } \\
\text { des nutriments ; et des services culturels comme } \\
\text { les avantages récréatifs, spirituels, religieux et } \\
\text { autres avantages non matériels. }\end{array}$ & $\begin{array}{l}\text { De nombreuses aires protégées } \\
\text { fournissent aussi des services } \\
\text { écosystémiques : p.ex. le Gunung } \\
\text { Gede National Park à Java, Indonésie } \\
\text { (catégorie II), qui aide à fournir de l'eau } \\
\text { douce à Jakarta et le Parc National des } \\
\text { Sundarbans, au Bengladesh (catégorie } \\
\text { IV) qui aide à protéger la côte contre les } \\
\text { inondations. }\end{array}$ \\
\hline
\end{tabular}


Tableau 1. Explication de la définition d'une aire protégée (suite)

\begin{tabular}{|c|c|c|}
\hline Phrase & Explication & Exemples et nouveaux détails \\
\hline $\begin{array}{l}\text { Valeurs } \\
\text { culturelles }\end{array}$ & $\begin{array}{l}\text { Inclut celles qui n'interfèrent pas avec le résultat de } \\
\text { la conservation (toutes les valeurs culturelles d'une } \\
\text { aire protégée devraient répondre à ce critère), y } \\
\text { compris en particulier: } \\
\text { - celles qui contribuent aux résultats de la } \\
\text { conservation (p.ex. les pratiques de gestion } \\
\text { traditionnelles dont les espèces clés sont } \\
\text { devenues tributaires); } \\
\text { - celles qui sont elles-mêmes menacées. }\end{array}$ & $\begin{array}{l}\text { De nombreuses aires protégées } \\
\text { contiennent des sites sacrés : ex. le } \\
\text { Nyika National Park au Malawi qui a } \\
\text { un bassin, une chute et une montagne } \\
\text { sacrés. La gestion traditionnelle des } \\
\text { forêts qui ont fourni le bois pour la } \\
\text { construction de temples, au Japon, a } \\
\text { donné certaines des plus anciennes } \\
\text { forêts du pays, comme la forêt primaire } \\
\text { protégée à l'extérieur de Nara. Les } \\
\text { forêts de Kaya sur la côte kényane } \\
\text { sont protégées aussi bien pour leur } \\
\text { biodiversité que pour leurs valeurs } \\
\text { culturelles. }\end{array}$ \\
\hline
\end{tabular}

\section{Les aspects tridimensionnels des aires protégées}

Dans certaines situations, les aires protégées doivent prendre en compte l'impact des activités humaines en trois dimensions. Les questions peuvent inclure p.ex. : protéger l'espace au-dessus d'une aire protégée contre toute perturbation d'avions passant à basse altitude, des vols d'hélicoptères ou de montgolfières, et limiter toute activité humaine sous sa surface, comme l'exploitation minière ou toute autre activité extractive. Les problèmes qui concernent spécifiquement les eaux marines ou intérieures incluent la pêche, le dragage, la plongée et le bruit sous la surface. Un certain nombre de pays ont inscrit cet aspect tridimensionnel dans leur législation sur les aires protégées ; p.ex. Cuba qui interdit l'exploitation minière sous des aires protégées. L'UICN encourage les gouvernements à envisager une disposition légale générale pour protéger les aires protégées des activités intrusives au-dessus et/ou en dessous du sol et sous l'eau. Elle encourage les gouvernements à s'assurer que des évaluations soient faites pour vérifier les effets potentiels de telles activités avant de prendre une décision quant à leur autorisation et, le cas échéant, quant aux limites ou aux conditions particulières qu'il faut fixer.

\section{Principes}

La définition devrait être appliquée dans le contexte d'un ensemble de principes connexes, soulignés ci-dessous

- Pour l'UICN, seules les aires dont le principal objectif est de conserver la nature peuvent être considérées comme des aires protégées ; cela peut inclure de nombreuses aires qui ont aussi d'autres buts de même importance, mais en cas de conflit, la conservation de la nature sera prioritaire ;
- Les aires protégées doivent empêcher, ou éliminer si nécessaire, toute exploitation ou pratique de gestion qui serait préjudiciable à leurs objectifs de départ ;

- Le choix de la catégorie devrait se fonder sur l(es) objectif(s) premier(s) déclaré(s) pour chaque aire protégée ;

- Le système ne se veut pas hiérarchique ;

- Toutes les catégories apportent une contribution à la conservation mais il faut choisir les objectifs en fonction de la situation considérée; les catégories ne sont pas toutes aussi utiles dans chaque situation;

- Toute catégorie peut exister avec tout type de gouvernance et vice versa ;

- Il faudrait encourager toute une variété d'approches de gestion dans la mesure où elles reflètent les nombreuses façons dont les communautés, de par le monde, expriment la valeur universelle du concept d'aire protégée ;

- La catégorie doit être changée si une évaluation montre que les objectifs déclarés de gestion à long terme ne correspondent pas à ceux de la catégorie attribuée ;

- Cependant, la catégorie n'est pas le reflet de l'efficacité de gestion ;

- Les aires protégées devraient normalement préserver ou, idéalement, accroître le caractère naturel de l'écosystème à protéger ;

- La définition et les catégories d'aires protégées ne doivent pas être utilisées comme une excuse pour déposséder des personnes de leurs terres.

\section{Définition d'un système d'aires protégées et l'approche par écosystème}

Les catégories doivent être appliquées dans le contexte des systèmes d'aires protégées nationaux ou autres et dans le cadre d'une approche par écosystème 
L'UICN insiste sur le fait que les aires protégées ne doivent pas être considérées comme des entités isolées mais comme faisant partie de paysages de conservation plus vastes, qui comprennent tant des systèmes d'aires protégées que des approches de la conservation par écosystème plus larges, qui sont mises en œuvre dans tout le paysage terrestre ou marin. La section suivante donne des définitions résumées de ces deux concepts.

\section{Système d'aires protégées}

La raison d'être primordiale d'un système d'aires protégées est d'augmenter l'efficacité de la conservation in-situ de la biodiversité. L'UICN suggère que la réussite à long terme de la conservation in-situ exige que le système mondial d'aires protégées comprenne un exemple représentatif de chaque écosystème différent du monde (Davey 1998). La CMAP/UICN caractérise un système d'aires protégées par le fait qu'il possède cinq éléments liés (Davey 1998 avec ajouts) :

- Représentativité, globalité et équilibre : comprend les meilleurs exemples de la gamme complète des types d'environnement dans un pays ; inclut la mesure dans laquelle les aires protégées offrent un échantillonnage équilibré des types d'environnement qu'elles sont censées représenter.

- Pertinence : intégrité, étendue spatiale et disposition des unités constituantes suffisantes pour, avec une gestion efficace, supporter la viabilité des processus environnementaux et/ou des espèces, des populations et des communautés qui constituent la biodiversité du pays ;

- Cohésion et complémentarité : contribution positive de chaque aire protégée à l'ensemble des objectifs de conservation et de développement durable définis pour le pays.

- Cohérence : application normalisée des objectifs de gestion, des politiques et des classifications dans des conditions comparables, de sorte que la raison d'être de chaque aire protégée d'un système est claire pour tous et que l'on maximise les chances que la gestion et l'utilisation appuient les objectifs.

- Rentabilité, efficience et équité : un équilibre approprié entre les coûts et les bénéfices, et une équité adéquate dans leur répartition; ceci inclut l'efficience : le nombre et la surface minimum des aires protégées nécessaires pour atteindre les objectifs du système.

En 2004, le Programme de travail sur les aires protégées de la $\mathrm{CDB}$ a fourni quelques critères pour les systèmes d'aires protégées dans l'objectif général du Programme pour établir et préserver " des systèmes nationaux et régionaux d'aires protégées qui soient globaux, efficacement gérés et écologiquement représentatifs".

\section{Approches par écosystème}

LUUCN croit que les aires protégées devraient être intégrées dans des systèmes d'aires protégées cohérents, et que ces systèmes devraient eux-mêmes être inclus dans des approches plus larges de la conservation et de l'utilisation de la terre et de l'eau, qui comprennent aussi bien la protection de la terre et de l'eau qu'une large variété d'approches de gestion durable. Ceci est dans la lignée des Principes du Malawi de la CDB (CDB/COP4, 1998), qui soulignent l'importance des stratégies d'utilisation durable. Ces stratégies de conservation à plus large échelle sont appelées selon les cas " approches à l'échelle du paysage ", " approches biorégionales " ou encore " approches par écosystème ». Lorsque ces approches comprennent la conservation d'aires qui connectent des aires protégées entre elles, les termes " conservation de la connectivité " sont utilisés. Chaque aire protégée particulière devrait, dès lors, autant que possible, contribuer à l'ensemble des aires protégées nationales et régionales ainsi qu'aux plans de conservation à grande échelle.

L'approche par écosystème est un cadre plus large pour planifier et développer la conservation et l'aménagement des terres et des eaux de façon intégrée. Dans ce contexte, les aires protégées sont un outil important - peut-être le plus important de tous.

La CDB définit l'approche par écosystème comme : "une stratégie pour la gestion intégrée des terres, de l'eau et des ressources vivantes, qui favorise la conservation et l'utilisation durable d'une manière équitable..." (CDB 2004).

\section{Catégories}

Chaque catégorie est décrite sous une série d'en-tête :

- Objectif(s) premier(s)

- Autres objectifs

- Caractéristiques marquantes

- Rôle dans le paysage terrestre ou marin

- Ce qui rend la catégorie unique

- Questions à considérer 


\section{Noms des aires protégées}

Le système des catégories fut introduit en grande partie pour aider à standardiser les descriptions de ce qui constitue une aire protégée particulière. Le nom des aires protégées, sauf celles de la catégorie II, a été choisi pour décrire, plus ou moins précisément, le principal objectif de gestion de la catégorie.

Les termes « Parc national », qui existaient bien avant le système des catégories, se sont avérés décrire particulièrement bien les grandes aires protégées de la catégorie II. II est vrai, cependant, que de nombreux parcs nationaux existants de par le monde ont des buts très différents de ceux qui sont définis par la catégorie II. En réalité, certains pays ont classé leurs parcs nationaux dans d'autres catégories de I'UICN (voir tableau 2, ci-dessous).

Tableau 2. «Parcs nationaux » dans diverses catégories

\begin{tabular}{|l|l|l|c|l|}
\hline Catégorie & Nom & Localisation & Taille (ha) & Date \\
\hline Ia & Dipperu National Park & Australie & 11.100 & 1969 \\
\hline II & Guanacaste National Park & Costa Rica & 32.512 & 1991 \\
\hline III & Yozgat Camligi National Park & Turquie & 264 & 1988 \\
\hline IV & Pallas Ounastunturi National Park & Finlande & 49.600 & 1938 \\
\hline V & Snowdonia National Park & Pays de Galles (GB) & 214.200 & 1954 \\
\hline VI & Expedition National Park & Australie & 2.930 & 1994 \\
\hline
\end{tabular}

II est important de noter que le fait qu'un pays ait appelé, ou veuille appeler une aire " parc national » ne signifie pas qu'elle doit être gérée selon les lignes directrices de la catégorie II. II faut, au contraire, identifier et appliquer le système de gestion le plus opportun ; le choix du nom relève de la décision des gouvernements et des autres parties prenantes.

Ce qui suit est un cadre. Bien que certaines aires protégées tombent tout naturellement dans l'une ou l'autre catégorie, dans d'autres cas, la distinction est moins évidente et elle nécessitera une analyse en profondeur des options possibles. Comme le classement dans une catégorie dépend des objectifs de gestion, cela dépendra plus de ce que l'autorité de gestion $a$ l'intention de faire pour le site que de tout ensemble de critères strict et inviolable. Il existe des outils pour aider au choix de la catégorie, mais souvent, la décision finale sera une question de jugement collectif.

De plus, comme le système est mondial, il est aussi inévitablement assez général. L'UICN encourage les pays à ajouter des détails supplémentaires à la définition des catégories pour répondre à leurs conditions propres si cela peut les aider, tout en restant dans le cadre des lignes directrices décrites plus bas. Plusieurs pays l'ont déjà fait ou sont entrain de le faire, et l'UICN les y encourage.

\section{Paysages naturels et culturels, terrestres et marins}

Nous relevons que peu, voire pas, de surfaces terrestres, d'eaux intérieures ou de côtes sont restées intouchées par l'activité humaine ; celle-ci a aussi affecté les océans du monde entier par la pression de la pêche et la pollution. Si les impacts de la pollution aérienne transfrontalière et les changements climatiques sont pris en compte, c'est toute la planète qui a été modifiée. II s'ensuit donc que des termes tels que " naturel » et « culturel » sont des approximations. Dans une certaine mesure, nous pouvons dire que toutes les aires protégées existent dans des paysages "culturels ", en ceci que des pratiques culturelles en auront changé et influencé l'écologie, souvent pendant des millénaires. Cependant, ceci est peu utile pour distinguer entre des types très différents de fonctionnement de l'écosystème. Nous utilisons donc les termes comme suit :

Les aires naturelles ou inaltérées sont celles qui conservent encore un ensemble complet ou presque complet d'espèces natives de l'endroit, au sein d'un écosystème qui fonctionne de manière plus ou moins naturelle.

Les aires culturelles ont subi des changements plus substantiels dus, par exemple, à l'agriculture sédentaire, au pâturage intensif permanent et à la gestion forestière qui ont altéré la composition ou la structure de la forêt. La composition des espèces et le fonctionnement de l'écosystème sont susceptibles d'avoir été notablement altérés. Les paysages culturels peuvent toutefois contenir encore une riche diversité d'espèces et, dans certains cas, celles-ci peuvent même être devenues dépendantes de la gestion culturelle.

L'emploi de termes comme " naturel » et « inaltéré » ne cherche pas à cacher ou à nier la longue gouvernance du terroir par des populations autochtones et traditionnelles, lorsqu'elles existent ; en réalité, de nombreuses aires ont gardé leur riche biodiversité précisément grâce à cette forme de gestion. 


\section{Objectifs communs aux six catégories d'aires protégées}

La définition implique un ensemble d'objectifs communs pour les aires protégées ; les catégories, elles, définissent des différences dans les approches de gestion. Les objectifs suivants devraient, ou peuvent s'appliquer à toutes les catégories d'aires protégées : c.-à-d. qu'ils ne permettent pas de distinguer les catégories l'une de l'autre.

\section{Toutes les aires protégées devraient viser à :}

- Conserver la composition, la structure, la fonction et le potentiel évolutif de la biodiversité ;

- Contribuer aux stratégies régionales de conservation (réserves centrales, zones tampons, corridors, étapes-relais pour les espèces migratrices, etc.) ;

- Préserver la biodiversité du paysage ou de l'habitat, des espèces et des écosystèmes associés ;

- Etre suffisamment grandes pour garantir l'intégrité et le maintien à long terme des cibles de conservation spécifiées, ou pouvoir être étendues pour y arriver ;

- Préserver à jamais les valeurs pour lesquelles elles ont été créées ;

- Opérer avec l'assistance d'un plan de gestion, ainsi que d'un programme de suivi et d'évaluation qui encourage une gestion adaptative ;

- Posséder un système de gouvernance clair et équitable.

\section{Lorsque cela est approprié, ${ }^{2}$ toutes les aires protégées devraient aussi viser à :}

- Préserver les caractéristiques significatives du paysage, sa géomorphologie et sa géologie ;

- Fournir des services écosystémiques régulateurs, y compris l'effet tampon contre les impacts des changements climatiques ;
- Conserver les zones naturelles et scéniques d'importance nationale et internationale à des fins culturelles, spirituelles et scientifiques ;

- Distribuer aux communautés locales et résidentes des bénéfices en accord avec les autres objectifs de la gestion ;

- Offrir des avantages récréatifs dans le respect des autres objectifs de la gestion ;

- Faciliter les activités de recherche scientifique qui ont un faible impact, et un suivi écologique lié et cohérent par rapport aux valeurs de l'aire protégée ;

- Utiliser les stratégies de gestion adaptative pour améliorer peu à peu l'efficacité de gestion et la qualité de la gouvernance ;

- Aider à fournir des opportunités éducatives (y compris au sujet des approches de gestion) ;

- Aider à gagner le support général à la protection.

Il faut noter que les membres de l'UICN ont adopté une recommandation lors du Congrès mondial de la conservation à Amman, en Jordanie, en octobre 2000, qui suggérait que l'exploitation minière ne devait pas se faire dans les aires protégées des catégories I à IV de l'UICN. La recommandation 2.82 comprend une section qui : "demande à tous les Etats membres de l'UICN d'interdire par la loi toutes les activités de prospection et d'exploitation des ressources minérales dans les aires protégées correspondant aux catégories I à $I V »$. La recommandation comprend aussi un paragraphe lié aux aires protégées des catégories V et VI : «... que dans les catégories Vet VI, la prospection et l'exploitation localisée ne soient acceptées que lorsqu'il est évident, compte-tenu de la nature et de la portée des activités proposées, que celles-ci sont compatibles avec les objectifs des aires protégées". Ceci est une recommandation qui n'est en aucune façon contraignante pour les gouvernements ; actuellement, certains interdisent l'activité minière dans les aires protégées de catégories I-IV, d'autres ne le font pas.

\footnotetext{
Cette distinction est faite parce que toutes les aires protégées n'offrent pas une géologie intéressante, des services écosystémiques ou des opportunités pour les moyens de subsistance locaux, etc. de sorte que ces objectifs ne sont pas universels, mais qu'ils sont appropriés le cas échéant. Les pages suivantes décrivent les caractéristiques distinctes de chaque catégorie de gestion qui s'ajoutent à ces buts premiers. Dans certains cas, un objectif tel que la recherche scientifique ou les loisirs pourra être mentionné parce que c’est un but majeur dans une catégorie donnée.
} 


\section{Catégorie la : Réserve naturelle intégrale}

La catégorie la contient des aires protégées qui sont mises en réserve pour protéger la biodiversité et aussi, éventuellement, des caractéristiques géologiques/ géomorphologiques, où les visites, l'utilisation et les impacts humains sont strictement contrôlés et limités pour garantir la protection des valeurs de conservation. Ces aires protégées peuvent servir d'aires de référence indispensables pour la recherche scientifique et la surveillance continue.

Avant de choisir une catégorie, il faut d'abord vérifier que le site répond à la définition d'une aire protégée (page 10).

\section{Objectif premier}

- Conserver les écosystèmes exceptionnels au niveau régional, national ou mondial, les espèces (individuelles ou en groupes) et/ou les caractéristiques de la géodiversité : ces caractères distinctifs auront été formés principalement ou entièrement par des forces non humaines et seraient dégradés ou détruits par tout impact humain sauf très léger.

\section{Autres objectifs}

- Conserver les écosystèmes, les espèces et les caractéristiques de la géodiversité dans un état aussi préservé de toute nouvelle activité humaine que possible ;

- Conserver des milieux naturels exemplaires à des fins d'études scientifiques, de suivi de l'environnement et d'éducation à l'environnement, y compris des aires de référence en excluant toute intrusion évitable ;

- Réduire au minimum les perturbations en planifiant et en menant avec circonspection les activités autorisées, de recherche et autres ;

- Conserver les valeurs culturelles et spirituelles associées à la nature.

\section{Caractéristiques marquantes}

L'aire doit généralement :

- Posséder un ensemble presque complet des espèces indigènes auxquelles on peut s'attendre et dont la densité est écologiquement significative, ou être à même de les rétablir à une telle densité par des processus naturels ou par des interventions de courte durée ;

- Posséder un ensemble complet d'écosystèmes indigènes, largement intacts, avec des processus écologiques intacts, ou qu'il est possible de restaurer avec un minimum d'intervention de gestion ;

- Etre à l'abri de toute intervention humaine directe qui compromettrait les objectifs de conservation spécifiés pour l'aire, ce qui implique habituellement de limiter l'accès des gens et d'exclure toute installation ;
- Ne pas exiger d'intervention substantielle ou permanente pour atteindre les objectifs de conservation ;

- Etre entourée, si possible, par des terres exploitées d'une façon qui contribue à l'atteinte des objectifs spécifiés de la conservation de l'aire ;

- Convenir comme site de référence pour la surveillance continue, afin de suivre l'impact relatif des activités humaines ;

- Etre gérée de façon à avoir relativement peu de visites ;

- Pouvoir être gérée de façon à garantir que les perturbations seront minimales (spécialement pertinent pour les environnements marins).

L'aire pourrait avoir une importance religieuse ou spirituelle (comme un site naturel sacré) pour autant que la conservation de la biodiversité soit identifiée comme un objectif premier. Dans ce cas, l'aire peut contenir des sites qui peuvent être visités par un nombre limité de personnes engagées dans des activités liées à leurs croyances et qui respectent les objectifs de la gestion de l'aire.

\section{Rôle dans le paysage terrestre ou marin}

Les aires de la catégorie Ia sont une composante vitale de la boîte à outils de la conservation. Alors que la terre est de plus en plus influencée par les activités humaines, il reste de moins en moins d'aires où ces activités sont strictement limitées. Sans la protection qui accompagne la désignation, il ne resterait bientôt plus d'aires de ce type. En tant que telles, ces aires contribuent significativement à la conservation en :

- Protégeant certaines des richesses de la terre qui ne survivront pas en dehors de ces endroits strictement protégés ;

- Fournissant des points de référence qui constituent une ligne de base et permettent d'effectuer des mesures et le suivi à long terme de l'impact des changements induits par l'homme en dehors de ces aires (ex. la pollution) ;

- Fournissant des lieux où les écosystèmes peuvent être étudiés dans un environnement aussi intact que possible ;

- Protégeant les services écosystémiques associés ;

- Protégeant les sites naturels qui ont aussi une importance religieuse et culturelle.

\section{Qu'est-ce qui fait que la catégorie la est unique ?}

Le classement dans une catégorie est une affaire de choix, qui dépend des objectifs de gestion à long terme, avec souvent un certain nombre d'options qui pourraient s'appliquer à n'importe quel site. Le tableau suivant résume certaines des principales raisons de choisir la catégorie Ia dans des situations spécifiques par rapport à d'autres catégories qui poursuivent des objectifs similaires. 


\begin{tabular}{|c|c|}
\hline \multicolumn{2}{|c|}{$\begin{array}{l}\text { La catégorie la diffère des autres des façons } \\
\text { suivantes: }\end{array}$} \\
\hline $\begin{array}{l}\text { Catégorie } \\
\text { lb }\end{array}$ & $\begin{array}{l}\text { Les aires protégées de la catégorie } \\
\text { lb seront généralement plus vastes et } \\
\text { protégées moins strictement des visites } \\
\text { que celles de la catégorie la : bien } \\
\text { qu'elles ne soient normalement pas l'objet } \\
\text { d'un tourisme de masse, elles peuvent } \\
\text { s'ouvrir à un certain nombre de visiteurs } \\
\text { prêts à voyager de façon autonome, par } \\
\text { exemple à pied ou en bateau, ce qui n'est } \\
\text { pas toujours le cas dans la catégorie la. }\end{array}$ \\
\hline $\begin{array}{l}\text { Catégorie } \\
\text { II }\end{array}$ & $\begin{array}{l}\text { Les aires protégées de la catégorie II } \\
\text { combinent d'habitude la protection de } \\
\text { l'écosystème aux loisirs, moyennant un } \\
\text { zonage, à une échelle qui ne convient } \\
\text { pas à la catégorie I. }\end{array}$ \\
\hline $\begin{array}{l}\text { Catégorie } \\
\text { III }\end{array}$ & $\begin{array}{l}\text { Les aires protégées de la catégorie } \\
\text { III sont généralement centrées sur un } \\
\text { élément naturel particulier, de sorte que } \\
\text { l'attention première de la gestion se porte } \\
\text { sur le maintien de cet élément alors que } \\
\text { les objectifs de la catégorie la concernent } \\
\text { généralement l'ensemble de l'écosystème } \\
\text { et de ses processus. }\end{array}$ \\
\hline $\begin{array}{l}\text { Catégorie } \\
\text { IV }\end{array}$ & $\begin{array}{l}\text { Les aires protégées de la catégorie IV } \\
\text { protègent des fragments d'écosystèmes } \\
\text { ou d'habitats qui requièrent souvent une } \\
\text { intervention continue de la gestion pour } \\
\text { subsister. Celles de la catégorie la, par } \\
\text { contre, doivent être largement autonomes, } \\
\text { et leurs objectifs excluent une telle } \\
\text { activité de gestion, tout comme le taux } \\
\text { de visites admis dans la catégorie IV. Les } \\
\text { aires protégées de la catégorie IV sont } \\
\text { aussi souvent créées pour protéger des } \\
\text { espèces ou des habitats particuliers plutôt } \\
\text { que poursuivre les objectifs écologiques } \\
\text { spécifiques de la catégorie la. }\end{array}$ \\
\hline $\begin{array}{l}\text { Catégorie } \\
\text { V }\end{array}$ & $\begin{array}{l}\text { Les aires protégées de la catégorie } V \text { sont } \\
\text { généralement des paysages terrestres } \\
\text { ou marins culturels qui ont été modifiés } \\
\text { par les hommes au cours des siècles ou } \\
\text { même des millénaires et qui exigent des } \\
\text { interventions constantes pour préserver } \\
\text { leurs qualités, y compris leur biodiversité. } \\
\text { De nombreuses aires protégées de la } \\
\text { catégorie V contiennent des installations } \\
\text { humaines permanentes. Tout cela est } \\
\text { incompatible avec la catégorie la. }\end{array}$ \\
\hline $\begin{array}{l}\text { Catégorie } \\
\text { VI }\end{array}$ & $\begin{array}{l}\text { Les aires protégées de la catégorie VI } \\
\text { contiennent des aires naturelles où la } \\
\text { conservation de la biodiversité est liée } \\
\text { à l'utilisation durable des ressources } \\
\text { naturelles, ce qui est incompatible } \\
\text { avec la catégorie la. Cependant, des } \\
\text { grandes aires protégées de la catégorie } \\
\text { VI peuvent renfermer des aires de la } \\
\text { catégorie la à l'intérieur de leurs limites, } \\
\text { suite à un zonage de la gestion. }\end{array}$ \\
\hline
\end{tabular}

\section{Questions à considérer}

- Il existe peu d'aires, dans le monde terrestre ou marin, qui ne portent pas les marques d'une activité humaine antérieure, même si, dans la plupart des cas, les anciens occupants ne sont plus là. Souvent, les aires protégées de la catégorie Ia requièrent donc un processus de restauration. Celle-ci doit se faire par des processus naturels ou des interventions limitées. Si une intervention continue est nécessaire, l'aire sera mieux à sa place dans une autre catégorie, comme la IV ou la V.

- Il existe peu d'aires qui ne soient pas sous l'une ou l'autre forme de propriété juridique ou traditionnelle, de sorte que trouver des espaces qui excluent toute activité humaine est souvent problématique.

- Certaines activités humaines ont une portée régionale et mondiale qui n'est pas arrêtée par les limites d'une aire protégée. Ceci est particulièrement évident avec la pollution atmosphérique et le climat, ou encore avec les nouvelles maladies émergentes. Dans une écologie toujours altérée davantage, il peut devenir de plus en plus difficile de préserver des aires intactes par la non-intervention.

- De nombreux sites naturels sacrés sont gérés de façon analogue aux aires protégées de la catégorie Ia pour des raisons spirituelles et culturelles et ils peuvent se trouver $\grave{a}$ l'intérieur d'aires protégées des catégories V ou VI.

\section{Catégorie lb : Zone de nature sauvage}

Les aires protégées de la catégorie lb sont généralement de vastes aires intactes ou légèrement modifiées, qui ont conservé leur caractère et leur influence naturels, sans habitations humaines permanentes ou significatives, qui sont protégées et gérées aux fins de préserver leur état naturel.

Avant de choisir une catégorie, il faut d'abord vérifier que le site répond à la définition d'une aire protégée (page 10).

\section{Objectif premier}

- Protéger à long terme l'intégrité écologique d'aires naturelles qui n’ont pas été modifiées par des activités humaines importantes, dépourvues d'infrastructures modernes, et où les forces et les processus naturels prédominent, pour que les générations actuelles et futures aient la possibilité de connaître de tels espaces.

\section{Autres objectifs}

- Prévoir l'accès du public à un rythme et d'une façon tels qu'il préservera les qualités sauvages de l'aire pour les générations présentes et futures ;

- Permettre aux communautés autochtones de conserver leur style de vie et leurs coutumes traditionnels, basés sur la nature sauvage, en vivant en faible densité et en utilisant les ressources disponibles d'une façon compatible avec les objectifs de la conservation ; 
- Protéger les valeurs culturelles et spirituelles et les avantages non matériels pertinents pour les populations autochtones ou non, comme la solitude, le respect des sites sacrés, le respect des ancêtres, etc.

- Permettre les activités scientifiques et éducatives peu invasives, qui ont un impact minime, lorsque ces activités ne peuvent pas se faire en dehors de la zone de nature sauvage.

\section{Caractéristiques marquantes}

L'aire doit généralement :

- Etre dépourvue de toute infrastructure moderne, de développement et de toute activité extractive industrielle, y compris - mais la liste n'est pas limitative - les routes, les pipelines, les lignes électriques, les antennes-relais pour les téléphones portables, les plates-formes pétrolières ou gazières, les terminaux offshore de gaz naturel liquéfié, toutes autres structures permanentes, tout développement minier ou hydroélectrique, toute extraction gazière et pétrolière, toute agriculture, $y$ compris le pâturage intensif du bétail, la pêche commerciale, les avions volant à basse altitude, etc., et, de préférence, les accès motorisés y sont fortement réduits ou totalement interdits.

- Secaractériser par un haut degré d'intégrité: elle contient un grand pourcentage de l'étendue originale de l'écosystème, des associations complètes ou presque de la faune et de la flore natives; elle conserve des systèmes proies-prédateurs intacts, y compris des grands mammiferes.

- Etre suffisamment étendue pour protéger la biodiversité ; pour préserver les processus écologiques et les services environnementaux ; pour conserver des refuges écologiques ; pour jouer un rôle tampon contre les impacts des changements climatiques; et pour garder les processus évolutifs.

- Offrir des opportunités exceptionnelles de solitude, goûtées dès que l'aire est atteinte, grâce à des moyens de transport simples, silencieux et non intrusifs (c.-à-d. des accès non motorisés ou des accès motorisés très réglementés lorsque c'est absolument nécessaire et qui respectent les objectifs biologiques cités plus haut).

- Etre dépourvue de toute utilisation ou présence humaine inappropriée ou excessive qui réduirait les valeurs de la vie sauvage et qui, à terme, empêcherait une aire de respecter les critères biologiques et culturels énumérés ci-dessus. Cependant, la présence humaine ne devrait pas être le facteur déterminant pour décider si l'on crée une aire de catégorie Ia. Les objectifs clés sont l'intégrité biologique et l'absence d'infrastructures permanentes, d'industries extractives, d'agriculture, de pratiques motorisées et d'autres indicateurs de technologie moderne et de longue durée.

Cependant, cette catégorie peut aussi inclure ;

- Des aires quelque peu perturbées qui sont capables de retrouver un état sauvage et des aires plus petites qui pourraient s'étendre ou jouer un rôle important dans une stratégie de protection de la nature sauvage plus large, en tant que partie d'un système d'aires protégées qui inclut la nature sauvage, pour autant que les objectifs de gestion pour ces aires un peu perturbées ou plus petites correspondent, par ailleurs, aux objectifs cités plus haut.

Lorsque l'intégrité biologique d'une zone de nature sauvage est assurée et que l'objectif premier décrit plus haut est atteint, le point focal de la gestion de la zone de nature sauvage peut évoluer vers d'autres objectifs tels que la protection des valeurs culturelles ou récréatives, mais uniquement dans la mesure où l’objectif premier reste assuré.

\section{Rôle dans le paysage terrestre ou marin}

Les zones de nature sauvage jouent de multiples façons un rôle semblable à celui des parcs nationaux de la catégorie II étant donné qu'elles protègent de grands écosystèmes avec leur fonctionnement (ou au moins des aires où de nombreux aspects d'un écosystème peuvent s'épanouir). Leurs rôles particuliers comprennent :

- Protéger de vastes aires en grande partie intactes où les processus de l'écosystème, y compris l'évolution, peuvent se poursuivre sans entraves humaines telles que le développement ou le tourisme de masse ;

- Protéger des services écosystémiques compatibles;

- Protéger des espèces et des communautés écologiques particulières qui exigent des surfaces relativement étendues d'habitat non perturbé ;

- Fournir un réservoir de ces espèces pour aider à repeupler les aires qui entourent l'aire protégée et qui sont gérées de manière durable ;

- Offrir un espace à un nombre limité de visiteurs qui veulent jouir de la vie sauvage ;

- Offrir des possibilités de réponses au changement climatique, y compris un changement de biome. 


\section{Qu'est-ce qui fait que la catégorie lb est unique?}

\begin{tabular}{|c|c|}
\hline \multicolumn{2}{|c|}{$\begin{array}{l}\text { La catégorie lb diffère des autres catégories des } \\
\text { façons suivantes: }\end{array}$} \\
\hline $\begin{array}{l}\text { Catégorie } \\
\text { la }\end{array}$ & $\begin{array}{l}\text { Les aires protégées de la catégorie la sont } \\
\text { des aires protégées strictement protégées, } \\
\text { avec des visites limitées. Elles sont souvent } \\
\text { (mais pas toujours) relativement petites } \\
\text { contrairement à celles de la catégorie } \\
\text { lb. II n'y a normalement pas d'habitants } \\
\text { dans la catégorie la, mais l'utilisation par } \\
\text { les communautés locales et autochtones } \\
\text { est autorisée dans de nombreuses aires } \\
\text { protégées de la catégorie lb. }\end{array}$ \\
\hline $\begin{array}{l}\text { Catégorie } \\
\text { II }\end{array}$ & $\begin{array}{l}\text { Les aires protégées des catégories lb et II } \\
\text { ont souvent une taille comparable et leur } \\
\text { but de conserver des écosystèmes en bon } \\
\text { état de fonctionnement est semblable. } \\
\text { Mais alors que la catégorie II accepte (ou } \\
\text { prévoit d'accepter) la fréquentation de } \\
\text { visiteurs et les infrastructures nécessaires, } \\
\text { dans la catégorie Ib, l'utilisation par les } \\
\text { visiteurs est plus restreinte et se limite à } \\
\text { ceux qui ont les capacités et l'équipement } \\
\text { pour survivre sans assistance. }\end{array}$ \\
\hline & $\begin{array}{l}\text { La catégorie III est destinée à la protection } \\
\text { d'un élément naturel spécifique, ce qui } \\
\text { n'est pas l'objectif de la catégorie Ib. Les } \\
\text { aires protégées de la catégorie III sont } \\
\text { souvent assez petites et, comme dans la } \\
\text { catégorie II, elles visent à encourager les } \\
\text { visites, parfois en grand nombre ; les sites } \\
\text { lb, d'autre part, sont généralement plus } \\
\text { grands et découragent tous les visiteurs } \\
\text { qui ne seraient pas spécialistes. }\end{array}$ \\
\hline $\begin{array}{l}\text { Catégorie } \\
\text { IV }\end{array}$ & $\begin{array}{l}\text { Les aires protégées de la catégorie IV } \\
\text { sont normalement assez petites et ne } \\
\text { sont certainement pas des écosystèmes } \\
\text { en bon état de fonctionnement ; la plupart } \\
\text { requièrent des interventions régulières de } \\
\text { la gestion pour préserver leur biodiversité ; } \\
\text { toutes ces caractéristiques sont à l'opposé } \\
\text { des conditions imposées par la catégorie lb. }\end{array}$ \\
\hline $\begin{array}{l}\text { Catégorie } \\
\text { V }\end{array}$ & $\begin{array}{l}\text { Les aires protégées de la catégorie } V \\
\text { comprennent des paysages culturels } \\
\text { terrestres ou marins, formés par des } \\
\text { interventions humaines (souvent } \\
\text { prolongées) et contenant d'habitude des } \\
\text { communautés humaines assez importantes. } \\
\text { La catégorie lb doit être dans un état aussi } \\
\text { naturel que possible et ne contenir de } \\
\text { paysages culturels que si l'intention est de } \\
\text { les restaurer dans un état quasi naturel. }\end{array}$ \\
\hline
\end{tabular}

Catégorie $\quad$ La catégorie VI est fondée sur

VI l'instauration d'un zonage interne et de régimes de gestion pour encourager l'utilisation durable ; bien que les zones de nature sauvage incluent parfois une certaine utilisation durable par les populations autochtones, cet aspect est secondaire par rapport aux objectifs de gestion et n'en fait pas vraiment partie.

\section{Questions à considérer}

- Certaines zones de nature sauvage acceptent le pâturage de bétail conduit par des populations nomades, et il peut être nécessaire de faire la distinction entre pâturage intensif et extensif ; cependant, ceci posera un problème si la population veut augmenter la densité du bétail.

\section{Catégorie II : Parc national}

Les aires protégées de la catégorie II sont de vastes aires naturelles ou quasi naturelles mises en réserve pour protéger des processus écologiques de grande échelle, ainsi que les espèces et les caractéristiques des écosystèmes de la région, qui fournissent aussi une base pour des opportunités de visites de nature spirituelle, scientifique, éducative et récréative, dans le respect de l'environnement et de la culture des communautés locales.

Avant de choisir une catégorie, il faut d'abord vérifier que le site répond à la définition d'une aire protégée (page 10).

\section{Objectif premier}

- Protéger la biodiversité naturelle de même que la structure écologique et les processus environnementaux sous-jacents, et promouvoir l'éducation et les loisirs. ${ }^{3}$

\section{Autres objectifs}

- Gérer l'aire de façon à perpétuer, dans un état aussi naturel que possible, des exemples représentatifs de régions physiographiques, de communautés biotiques, de ressources génétiques et de processus naturels intacts ;

- Maintenir des populations viables et écologiquement opérationnelles et des assemblages d'espèces indigènes en densités suffisantes pour préserver à long terme l'intégrité et la résilience de l'écosystème ;

- Contribuer en particulier à la conservation d'espèces occupant de grands espaces, de processus écologiques régionaux et des voies de migration ;

\footnotetext{
3 Notons que le nom « parc national » n'est pas exclusivement lié à la catégorie II. Des endroits appelés parcs nationaux se trouvent dans toutes les catégories (et il y a même certains parcs nationaux qui ne sont pas du tout des aires protégées). Le nom est employé ici parce qu’il décrit les aires protégées de la catégorie II dans de nombreux pays. Le fait qu’une aire soit appelée parc national est indépendant de l'approche de sa gestion. En particulier, rappelons que les termes «parc national » ne doivent jamais être invoqués pour déposséder des gens de leurs terres.
} 
- Gérer la fréquentation de visiteurs à des fins spirituelles, éducatives, culturelles et récréatives de façon à ce qu'elle ne cause aucune dégradation biologique ou écologique significative des ressources naturelles;

- Prendre en compte les besoins des populations autochtones et des communautés locales, y compris l'utilisation de ressources de subsistance, dans la mesure où celles-ci n'ont pas d'incidence négative sur le premier objectif de gestion ;

- Contribuer à l'économie locale par le tourisme.

\section{Caractéristiques marquantes}

Les aires de la catégorie II sont normalement vastes et préservent le bon fonctionnement de l' " écosystème ", encore que, pour y arriver, elles peuvent devoir être complétées par une gestion compréhensive dans les régions voisines.

- L'aire devrait contenir des échantillons représentatifs des régions naturelles majeures, ainsi que des caractéristiques biologiques, panoramiques et environnementales les plus marquantes, où les espèces végétales et animales natives, les habitats et les sites géomorphologiques sont d'une importance spéciale du point de vue spirituel, scientifique, éducatif, récréatif ou touristique.

- L'aire doit être suffisamment vaste et d'une qualité écologique assez bonne pour préserver les fonctions et les processus écologiques qui permettront aux espèces et aux communautés indigènes de survivre à long terme avec un minimum d'interventions de gestion.

- La composition, la structure et la fonction de la biodiversité doivent être dans un état très proche de l'état « naturel » ou avoir la possibilité d'y revenir, avec un risque relativement bas d'invasions par des espèces exogènes.

\section{Rôle dans le paysage terrestre ou marin}

La catégorie II donne des opportunités de conservation à grande échelle là où les processus écologiques peuvent se poursuivre indéfiniment, fournissant de l'espace à la poursuite de l'évolution. Les parcs nationaux sont souvent des étapes relais clés pour la conception et la création de corridors biologiques à grande échelle ou pour toute autre initiative de maintien de la connectivité requise pour la conservation des espèces (qui ont besoin de grands espaces et/ou migratrices) qui ne peuvent pas être complètement préservées dans une seule aire protégée. Leur rôle clé est donc de :

- Protéger des processus écologiques à grande échelle qui ne pourraient pas être assurés par des aires protégées plus petites ou des paysages culturels ;

- Protéger des services écologiques compatibles ;

- Protéger des espèces et des communautés particulières qui exigent des espaces relativement vastes d'habitat non perturbé ;

- Fournir un réservoir de ces espèces pour aider à repeupler les aires gérées de façon durable qui entourent l'aire protégée ;

- Etre intégrées à l'aménagement du territoire terrestre ou aquatique qui les entoure pour contribuer aux projets de conservation de grande envergure ;
- Informer et intéresser les visiteurs aux besoins de programmes de conservation et à leur potentiel ;

- Encourager un développement économique compatible, principalement grâce aux loisirs et au tourisme, qui peut contribuer à l'économie locale et nationale et en particulier aux communautés locales.

Les aires de la catégorie II doivent être protégées plus strictement quand les fonctions écologiques et l'assemblage des espèces indigènes sont relativement intacts ; les paysages environnants peuvent connaître un degré variable d'utilisations consommatrices ou non, mais idéalement, ils devraient servir de zones tampons pour l'aire protégée.

\section{Qu'est-ce qui fait que la catégorie II est unique?}

\begin{tabular}{|c|c|}
\hline La catég & $\begin{array}{l}\text { Il diffère des autres catégories des } \\
\text { intes : }\end{array}$ \\
\hline $\begin{array}{l}\text { Catégorie } \\
\text { la }\end{array}$ & $\begin{array}{l}\text { La catégorie II n'est généralement } \\
\text { pas aussi strictement préservée que } \\
\text { la catégorie la et elle peut accepter } \\
\text { des infrastructures touristiques et des } \\
\text { visites. Cependant, les aires protégées } \\
\text { de la catégorie II ont souvent des zones } \\
\text { centrales où le nombre de visiteurs } \\
\text { est strictement contrôlé et qui peuvent } \\
\text { ressembler davantage à la catégorie la. }\end{array}$ \\
\hline $\begin{array}{l}\text { Catégorie } \\
\text { Ib }\end{array}$ & $\begin{array}{l}\text { Les visites dans la catégorie II seront } \\
\text { probablement assez différentes de celles } \\
\text { dans les zones de nature sauvage, avec } \\
\text { plus d'infrastructures associées (pistes, } \\
\text { routes, lodges, etc.) et elles seront donc } \\
\text { probablement plus nombreuses. Les aires } \\
\text { protégées de la catégorie II ont souvent des } \\
\text { zones centrales où le nombre de visiteurs } \\
\text { est strictement contrôlé et qui peuvent } \\
\text { ressembler davantage à la catégorie lb. }\end{array}$ \\
\hline $\begin{array}{l}\text { Catégorie } \\
\text { III }\end{array}$ & $\begin{array}{l}\text { La gestion dans la catégorie III est } \\
\text { destinée à la protection d'un élément } \\
\text { naturel spécifique, alors que dans la } \\
\text { catégorie II, elle s'attache à sauvegarder } \\
\text { un écosystème entier. }\end{array}$ \\
\hline $\begin{array}{l}\text { Catégorie } \\
\text { IV }\end{array}$ & $\begin{array}{l}\text { La catégorie II est destinée à maintenir } \\
\text { l'intégrité écologique à l'échelle de } \\
\text { l'écosystème, alors que la catégorie } \\
\text { IV vise à protéger des habitats et des } \\
\text { espèces individuelles. En pratique, les } \\
\text { aires protégées de la catégorie IV sont } \\
\text { rarement assez vastes pour protéger tout } \\
\text { un écosystème, et la distinction entre les } \\
\text { catégories II et IV est dans une certaine } \\
\text { mesure affaire de degré : les sites de } \\
\text { catégorie IV sont susceptibles d'être assez } \\
\text { petits (marais particuliers, fragments } \\
\text { de forêt, même s'il y a des exceptions) } \\
\text { alors que ceux de la catégorie II sont } \\
\text { susceptibles d'être beaucoup plus étendus } \\
\text { et au moins assez autonomes. }\end{array}$ \\
\hline
\end{tabular}




\begin{tabular}{|l|l|}
\hline $\begin{array}{l}\text { Catégorie } \\
\text { V }\end{array}$ & $\begin{array}{l}\text { Les aires protégées de la catégorie II sont } \\
\text { essentiellement des systèmes naturels } \\
\text { ou ils sont en voie de redevenir des } \\
\text { systèmes naturels alors que ceux de la } \\
\text { catégorie V sont des paysages culturels } \\
\text { qui ne visent qu'à rester tels. }\end{array}$ \\
\hline $\begin{array}{l}\text { Catégorie } \\
\text { VI }\end{array}$ & $\begin{array}{l}\text { La catégorie II ne permet généralement } \\
\text { pas d'utiliser les ressources sauf à des fins } \\
\text { de subsistance ou de récréation légère. }\end{array}$ \\
\hline
\end{tabular}

\section{Questions à considérer}

- Les concepts de ce qu'est la nature se développent rapidement, et certaines aires qui pouvaient jadis être considérées comme naturelles sont vues de plus en plus, dans une certaine mesure, comme des paysages culturels - p.ex. des paysages de savane où l'on a eu recours aux feux pour préserver la mosaïque de la végétation et donc les populations animales pour la chasse. La limite entre ce qui est considéré et géré comme la catégorie II et la catégorie V pourrait donc bien changer avec le temps.

- La commercialisation des espaces terrestres et aquatiques dans la catégorie II suscite des défis dans de nombreuses régions du monde, notamment en raison d'une perception politique du fait que des ressources seraient "bloquées " dans les parcs nationaux, d'une pression croissante pour davantage d'exploitations récréatives en dépit du manque de déontologie de tour-opérateurs, du développement de programmes d'aquaculture et de mariculture, et d'une tendance qui va vers la privatisation de telles aires.

- Les problèmes de populations installées dans les aires protégées qui sont proposées pour la catégorie II, les questions de déplacements, de compensations (y compris pour les communautés de pêcheurs déplacées des aires marines et côtières protégées), les options de moyens de subsistance alternatifs et des approches de gestion modifiées sont tous des thèmes émergents.

\section{Catégorie III : Monument ou élément naturel}

Les aires protégées de la catégorie III sont mises en réserve pour protéger un monument naturel spécifique, qui peut être un élément topographique, une montagne ou une caverne sous-marine, une caractéristique géologique telle qu'une grotte ou même un élément vivant comme un îlot boisé ancien. Ce sont généralement des aires protégées assez petites et elles ont souvent beaucoup d'importance pour les visiteurs.

Avant de choisir une catégorie, il faut d'abord vérifier que le site répond à la définition d'une aire protégée (page 10).

\section{Objectif premier}

- Protéger des éléments naturels exceptionnels spécifiques ainsi que la biodiversité et les habitats associés.

\section{Autres objectifs}

- Assurer la protection de la biodiversité dans des paysages terrestres ou marins qui ont, par ailleurs, subi des changements majeurs $;^{4}$

- Protéger des sites naturels spécifiques qui ont une valeur spirituelle et/ou culturelle, lorsqu'ils sont aussi importants pour la biodiversité ;

- Préserver les valeurs spirituelles et culturelles traditionnelles du site.

\section{Caractéristiques marquantes}

Les aires protégées de la catégorie III sont d'habitude des sites relativement petits centrés sur un ou plusieurs éléments naturels majeurs et l'écologie qui leur est associée, plutôt que sur un écosystème plus vaste. Elles sont gérées en grande partie comme celles de la catégorie II. Le terme " naturel » tel qu'il est utilisé ici peut faire référence à des éléments complètement naturels (l'utilisation la plus commune) mais aussi, parfois, à des éléments qui ont subi l'influence humaine. Dans ce dernier cas, les sites doivent toujours avoir d'importantes qualités en matière de biodiversité qui devraient se refléter comme une priorité dans les objectifs de gestion si l'on veut qu'ils soient classés comme aires protégées plutôt que comme des sites historiques ou spirituels. Les aires protégées de la catégorie III devraient inclure :

- Des éléments naturels géologiques et géomorphologiques : tels que chutes d'eau, falaises, cratères, grottes, gisements de fossiles, dunes de sable, formations rocheuses, vallées et éléments marins tels que montagnes sous-marines ou formations coralliennes ;

- Des éléments naturels influencés par la culture : comme des installations troglodytiques et d'anciennes pistes ;

- Des sites naturels culturels : comme les nombreuses formes de sites naturels sacrés (îlots forestiers sacrés, sources, montagnes, criques, etc.) importants pour un ou plusieurs groupes religieux ;

- Des sites culturels et l'écologie associée : là où la protection d'un site culturel protège aussi une biodiversité significative et importante, tels les sites archéologiques/historiques qui sont inextricablement liés à une aire naturelle.

La conservation de la nature dans les aires protégées de la catégorie III est de deux types principaux :

- La biodiversité qui est liée uniquement aux conditions écologiques associées à l'élément naturel - comme les

4 Remarquons que la protection de sites culturels spécifiques peut aussi souvent fournir des havres d'habitats naturels ou semi naturels dans des zones qui ont, par ailleurs, subi des modifications substantielles - p.ex. les anciens arbres autour des temples. 
endroits vaporisés par une chute d'eau, les conditions écologiques prévalant dans les grottes ou les espèces végétales confinées à des falaises.

- La biodiversité qui survit parce que la présence des valeurs spirituelles ou culturelles du site a préservé un habitat naturel ou semi-naturel dans ce qui serait sans cela un écosystème modifié - comme certains sites naturels ou historiques sacrés auxquels sont associées des aires naturelles. Dans ces cas, les critères clés pour leur admission comme aire protégée seront (i) la valeur du site en tant que contribution à la conservation à grande échelle et (ii) la priorité qu'aura la conservation de la biodiversité dans les plans de gestion.

La catégorie III a été suggérée, car elle recourt à une approche naturelle de la gestion, pour de nombreux sites naturels sacrés, tels que les îlots forestiers sacrés. Même s'il y a des sites naturels sacrés dans toutes les catégories et qu’ils peuvent bénéficier d'un large éventail d'approches de gestion, ils peuvent être particulièrement bien adaptés à une gestion en tant que monuments naturels.

\section{Rôle dans le paysage terrestre ou marin}

La catégorie III est réellement destinée à protéger ce qui est inhabituel plutôt qu'à fournir des composants logiques dans une approche à grande échelle de la conservation, de sorte que le rôle de ces éléments dans les stratégies par paysage ou écorégionales peut être parfois plus opportuniste que réellement planifié. Dans d'autres cas (p.ex. les réseaux de grottes), ces sites peuvent jouer un rôle écologique clé identifié dans les plans de conservation plus généraux. :

- Les monuments naturels importants peuvent parfois servir d'incitants pour la protection et d'opportunités pour l'éducation environnementale et culturelle, même dans des aires où d'autres formes de protection se voient repoussées par la pression de la population ou du développement ; c'est le cas de sites sacrés ou culturels importants, et dans ces cas-là, la catégorie III peut préserver des échantillons d'habitats naturels dans des paysages par ailleurs culturels, ou dans des paysages fragmentés.

\section{Qu'est-ce qui fait que la catégorie III est unique?}

Etant donné qu' elle est destinée à protéger un élément particulier, la catégorie III est peut-être, de toutes les catégories, celle qui est la plus influencée par la perception humaine de ce qui est important dans un paysage terrestre ou marin plutôt que par toute évaluation plus quantitative des valeurs. Ceci est moins applicable dans les aires protégées de la catégorie III désignées pour leurs caractéristiques géologiques, où une identification systématique est possible. La gestion est généralement plus centrée sur la protection et le maintien d'éléments naturels particuliers.
Le fait qu'une aire contienne un monument naturel important ne signifie pas qu'elle sera forcément gérée comme une aire de catégorie III ; par exemple, le Grand Canyon, en Arizona est géré comme une aire de catégorie II malgré le fait qu'il est un des plus fameux monuments naturels du monde, parce qu'il est aussi une aire vaste et variée, avec des activités récréatives qui en font une aire mieux adaptée au modèle de la catégorie II. La catégorie III convient mieux lorsque la protection de l'élément est l'objectif unique ou dominant.

\begin{tabular}{|c|c|}
\hline \multicolumn{2}{|c|}{$\begin{array}{l}\text { La catégorie III diffère des autres catégories des } \\
\text { façons suivantes : }\end{array}$} \\
\hline $\begin{array}{l}\text { Catégorie } \\
\text { la }\end{array}$ & \multirow{2}{*}{$\begin{array}{l}\text { La catégorie III n'est pas confinée aux } \\
\text { paysages naturels et intacts mais elle } \\
\text { peut être attribuée dans des aires qui } \\
\text { sont par ailleurs des paysages culturels } \\
\text { ou encore des paysages fragmentés. } \\
\text { Les visites et les loisirs sont souvent } \\
\text { encouragés, et le suivi se limite à la } \\
\text { bonne compréhension et à l'entretien d'un } \\
\text { élément naturel particulier. }\end{array}$} \\
\hline $\begin{array}{l}\text { Catégorie } \\
\text { lb }\end{array}$ & \\
\hline & $\begin{array}{l}\text { Dans la catégorie III, l'emphase n'est pas } \\
\text { placée sur la protection de l'ensemble } \\
\text { de l'écosystème mais sur des éléments } \\
\text { naturels particuliers; à part cela, la } \\
\text { catégorie III est semblable à la catégorie II } \\
\text { et est gérée de façon fort semblable, mais } \\
\text { à une échelle relativement plus petite, tant } \\
\text { en taille qu'en complexité de la gestion. }\end{array}$ \\
\hline & $\begin{array}{l}\text { L'emphase de la catégorie III n'est pas } \\
\text { mise sur la protection d'espèces ou } \\
\text { d'habitats clés mais sur des éléments } \\
\text { naturels particuliers. }\end{array}$ \\
\hline $\begin{array}{l}\text { Catégorie } \\
\text { V }\end{array}$ & $\begin{array}{l}\text { La catégorie III n'est pas limitée aux } \\
\text { paysages culturels, et les pratiques de } \\
\text { gestion se concentrent probablement } \\
\text { davantage sur la protection plus stricte de } \\
\text { l'élément particulier que dans le cas de la } \\
\text { catégorie V. }\end{array}$ \\
\hline $\begin{array}{l}\text { Catégorie } \\
\text { VI }\end{array}$ & $\begin{array}{l}\text { La catégorie III n'est pas destinée à } \\
\text { l'utilisation durable des ressources. }\end{array}$ \\
\hline
\end{tabular}

\section{Questions à considérer}

- Il sera parfois difficile de s'assurer des qualités de conservation des sites de la catégorie III, spécialement dans les cas où il peut y avoir des pressions pour faire accepter les sites dans un système d'aires protégées afin d'aider à en protéger les valeurs culturelles ou spirituelles.

- Tous les monuments naturels ne sont pas permanents - même si certains arbres sacrés survivent depuis mille ans ou plus, ils vont quand même mourir un jour - en fait certains arbres sont considérés comme sacrés en partie parce qu'ils sont déjà très vieux. Ce qui arrive à une aire protégée de catégorie III lorsque son monument naturel clé meurt ou se dégrade n'est pas clair.

- Il est parfois difficile de tracer la limite entre un monument naturel et un site culturel, spécialement lorsque des restes archéologiques sont inclus dans la catégorie III. 
- Certains " monuments " dits tels peuvent exiger la protection d'un écosystème plus vaste pour subsister - par exemple, une chute d'eau peut avoir besoin de la protection d'un bassin complet pour préserver son débit.

\section{Catégorie IV : Aire de gestion des habitats ou des espèces}

Les aires protégées de la catégorie IV visent à protéger des espèces ou des habitats particuliers, et leur gestion reflète cette priorité. De nombreuses aires protégées de la catégorie IV ont besoin d'interventions régulières et actives pour répondre aux exigences d'espèces particulières ou pour maintenir des habitats, mais cela n'est pas une exigence de la catégorie.

Avant de choisir une catégorie, il faut d'abord vérifier que le site répond à la définition d'une aire protégée (page 10).

\section{Objectif premier}

- Maintenir, conserver et restaurer des espèces et des habitats. ${ }^{5}$

\section{Autres objectifs}

- Protéger les formations végétales ou d'autres caractéristiques biologiques par des approches de gestion traditionnelles ;

- Protéger des fragments d'habitats comme composants de stratégies de conservation à l'échelle du paysage terrestre ou marin ;

- Développer l'éducation du public et son appréciation des espèces et /ou des habitats concernés ;

- Offrir un moyen qui permet aux résidents des villes d'être régulièrement en contact avec la nature.

\section{Caractéristiques marquantes}

Les aires protégées de la catégorie IV aident d'habitude à protéger ou à restaurer : (1) les espèces végétales d'importance internationale, nationale ou locale ; (2) des espèces animales d'importance internationale, nationale ou locale, y compris les espèces sédentaires ou migratrices; et/ou (3) des habitats. La taille des aires varie : elle peut être souvent relativement petite mais ceci n'est pas une caractéristique marquante. La gestion varie en fonction des besoins. La protection peut suffire pour préserver des espèces et/ou des habitats particuliers. Cependant, comme les aires protégées de la catégorie IV incluent souvent des fragments d'un écosystème, ces aires peuvent ne pas être auto-suffisantes et exiger des interventions actives et régulières de la gestion pour garantir la survie d'habitats spécifiques et/ou pour satisfaire aux exigences d'espèces particulières. Un certain nombre d'approches peuvent convenir :
- Protection d'une espèce particulière : pour protéger une espèce cible particulière qui sera habituellement menacée (p.ex. une des dernières populations restantes);

- Protection des habitats : pour préserver ou restaurer des habitats, qui sont souvent des fragments d'écosystèmes ;

- Gestion active pour préserver une espèce cible : pour préserver des populations viables d'espèces particulières, ce qui peut comprendre par exemple la création ou le maintien d'un habitat artificiel (comme la création d'un récif artificiel), la fourniture de compléments alimentaires, ou d'autres systèmes de gestion active ;

- Gestion active d'écosystèmes naturels ou semi-naturels : pour préserver des habitats naturels ou semi-naturels qui sont trop petits ou trop profondément altérés pour être auto-suffisants, p.ex. si les herbivores naturels sont absents, ils pourraient être remplacés par du bétail domestique ou par des coupes manuelles ; ou si l'hydrologie a été modifiée, il peut être nécessaire de recourir aux drainages ou à l'irrigation artificiels ;

- Gestion active d'écosystèmes définis par leurs qualités culturelles : pour maintenir des systèmes de gestion culturels lorsqu'ils sont associés à une biodiversité unique. L'intervention doit être continue parce que l'écosystème a été créé ou, au moins substantiellement modifié par la gestion. Le but premier de la gestion est le maintien de la biodiversité associée.

Une gestion active signifie que le fonctionnement général de l'écosystème est modifié, par exemple en stoppant la succession naturelle, en fournissant un complément alimentaire ou en créant des habitats artificiels ; c.-à-d. que la gestion va souvent inclure bien plus que la simple réponse aux menaces comme le braconnage ou les espèces invasives, étant donné que ces activités-là ont lieu dans pratiquement toutes les aires protégées de quelque catégorie que ce soit et qu'elles ne sont donc pas caractéristiques. Les aires protégées de la catégorie IV sont en général accessibles au public.

\section{Rôle dans le paysage terrestre ou marin}

Les aires protégées de la catégorie IV servent souvent à « boucher les trous " dans des stratégies de conservation en protégeant des espèces ou des habitats clés dans des écosystèmes. Elles peuvent, par exemple, servir à :

- Protéger des populations d'espèces en danger critique d'extinction qui requièrent des interventions de gestion particulières pour garantir leur survie ;

Ceci est un changement par rapport aux lignes directrices de 1994 qui définissaient la catégorie IV comme des aires protégées qui nécessitaient des interventions actives régulières de la gestion. Ce changement fut apporté parce que c'était la seule catégorie définie par le processus de gestion plutôt que par son objectif final et parce que, ce faisant, cela signifiait que de petites réserves destinées à protéger des habitats ou des espèces particuliers avaient tendance à rester en dehors du système de catégories. 
- Protéger des habitats rares ou menacés, y compris des fragments d'habitats ;

- Garantir des étapes-relais (endroits où les espèces migratrices peuvent se reposer et se nourrir) ou des sites de reproduction ;

- Fournir des stratégies et des options de gestion flexibles dans les zones tampons qui entourent - ou des corridors pour assurer la connectivité entre - des aires plus strictement protégées, et qui sont plus acceptables pour les communautés locales et pour d'autres parties prenantes;

- Préserver des espèces qui sont devenues dépendantes de paysages culturels là où leurs habitats d'origine ont disparu ou ont été altérés.

\section{Qu'est-ce qui fait que la catégorie IV est unique?}

La catégorie IV offre une approche de gestion, avec ou sans intervention, utilisée dans des aires qui ont déjà subi des modifications substantielles.

\begin{tabular}{|l|l|}
\hline $\begin{array}{l}\text { La catégorie IV diffère des autres catégories des } \\
\text { façons suivantes : }\end{array}$ \\
\hline $\begin{array}{l}\text { Catégorie } \\
\text { la }\end{array}$ & $\begin{array}{l}\text { Les aires protégées de la catégorie } \\
\text { IV ne sont pas strictement protégées } \\
\text { contre toute utilisation humaine; des } \\
\text { recherches peuvent s'y faire, mais c'est } \\
\text { généralement un objectif secondaire. }\end{array}$ \\
\hline $\begin{array}{l}\text { Catégorie } \\
\text { Ib }\end{array}$ & $\begin{array}{l}\text { Les aires protégées de la catégorie IV ne } \\
\text { peuvent pas être décrites comme des aires } \\
\text { « de nature sauvage ॥ telles que les définit } \\
\text { I'UICN. Nombre d'entre elles feront l'objet } \\
\text { d'interventions de gestion qui sont contraires } \\
\text { au concept de nature sauvage propre à la } \\
\text { catégorie lb ; et celles qui restent non gérées } \\
\text { sont susceptibles d'être trop petites pour } \\
\text { répondre aux objectifs de la catégorie Ib. }\end{array}$ \\
\hline $\begin{array}{l}\text { Catégorie } \\
\text { II }\end{array}$ & $\begin{array}{l}\text { Les aires protégées de la catégorie IV } \\
\text { orientent leur conservation vers des } \\
\text { espèces ou des habitats particuliers et } \\
\text { peuvent, par conséquent, accorder moins } \\
\text { d'importance à d'autres éléments de } \\
\text { l'écosystème, alors que les aires protégées } \\
\text { de la catégorie II visent à protéger des } \\
\text { écosystèmes fonctionnels complets. Les } \\
\text { catégories II et IV peuvent, dans certaines } \\
\text { circonstances, se ressembler beaucoup, } \\
\text { et la distinction porte, en partie, sur la } \\
\text { question de l'objectif - c.-à-d. si le but } \\
\text { est de protéger au maximum la totalité } \\
\text { de l'écosystème (catégorie II) ou s'il se } \\
\text { concentre sur la protection de quelques } \\
\text { espèces ou habitats clés (catégorie IV). }\end{array}$ \\
\hline
\end{tabular}

\begin{tabular}{|l|l|}
\hline Catégorie & $\begin{array}{l}\text { L'objectif des aires protégées de la } \\
\text { III } \\
\text { alorgorie IV est de nature plus biologique } \\
\text { centrée sur le site et plus orientée sur la } \\
\text { morphologie et la culture. }\end{array}$ \\
\hline Catégorie & $\begin{array}{l}\text { Les aires protégées de la catégorie IV } \\
\text { visent à protéger des espèces et des } \\
\text { habitats identifiés et ciblés alors que la } \\
\text { catégorie V veut protéger des habitats } \\
\text { terrestres ou marins complets comprenant } \\
\text { une valeur ajoutée pour la conservation } \\
\text { de la nature. Les aires protégées de } \\
\text { la catégorie V ont des caractéristiques } \\
\text { socioculturelles qui peuvent être } \\
\text { absentes dans la catégorie IV. Même si } \\
\text { les aires protégées de la catégorie IV } \\
\text { peuvent utiliser une approche de gestion } \\
\text { traditionnelle, ce sera explicitement pour } \\
\text { préserver des espèces associées dans } \\
\text { le cadre d'un plan de gestion et non, de } \\
\text { manière plus large, comme facette d'une } \\
\text { approche de gestion qui inclut une gamme } \\
\text { variée d'activités lucratives. }\end{array}$ \\
\hline Catégorie \\
VI & $\begin{array}{l}\text { Les interventions de gestion dans les } \\
\text { aires protégées de la catégorie IV } \\
\text { sont d'abord destinées à préserver } \\
\text { des espèces ou des habitats alors } \\
\text { que dans les aires protégées de la } \\
\text { catégorie VI, elles sont destinées à } \\
\text { relier la conservation de la nature à } \\
\text { l'utilisation durable des ressources. } \\
\text { Comme celles de la catégorie V, les } \\
\text { aires protégées de la catégorie VI sont } \\
\text { généralement plus grandes que dans } \\
\text { la catégorie IV. }\end{array}$ \\
\hline
\end{tabular}

\section{Questions à considérer}

- De nombreuses aires protégées de la catégorie IV se situent dans des paysages terrestres ou marins très peuplés, où la pression humaine est comparativement plus forte, que ce soit en termes d'utilisation illégale potentielle ou en nombre de visites.

- Les aires protégées de la catégorie IV qui s'appuient sur des interventions de gestion régulières ont besoin de ressources appropriées de la part de l'autorité de gestion et peuvent être relativement coûteuses à entretenir à moins que le travail ne soit réalisé bénévolement par les communautés locales ou par d'autres personnes.

- Comme elles protègent habituellement une partie d'écosystème, la réussite à long terme de la gestion des aires protégées de la catégorie IV nécessite un suivi attentif et un accent encore plus important que d'habitude sur des approches globales par écosystème et sur une gestion compatible dans d'autres parties du paysage terrestre ou marin. 


\section{Catégorie V : Paysage terrestre ou marin protégé}

Une aire protégée où l'interaction des hommes et de la nature a produit, au fil du temps, une aire qui possède un caractère distinct, avec des valeurs écologiques, biologiques, culturelles et panoramiques considérables, et où la sauvegarde de l'intégrité de cette interaction est vitale pour protéger et maintenir l'aire, la conservation de la nature associée ainsi que d'autres valeurs.

Avant de choisir une catégorie, il faut d'abord vérifier que le site répond à la définition d'une aire protégée (page 10).

\section{Objectif premier}

- Protéger et maintenir d'importants paysages terrestres ou marins, la conservation de la nature qui y est associée, ainsi que d'autres valeurs créées par les interactions avec les hommes et leurs pratiques de gestion traditionnelles.

\section{Autres objectifs}

- Préserver une interaction équilibrée entre la nature et la culture par la protection de paysages terrestres ou marins et par des approches de gestion des sociétés, des cultures et des valeurs spirituelles traditionnelles associées;

- Contribuer à la conservation à long terme en préservant les espèces associées aux paysages culturels et/ou en offrant des opportunités de conservation dans des paysages intensément utilisés ;

- Fournir des opportunités de distractions, de bien-être et d'activités socioéconomiques grâce aux loisirs et au tourisme ;

- Offrir des produits naturels et des services environnementaux ;

- Proposer un cadre pour étayer l'implication active de la communauté dans la gestion de paysages terrestres ou marins précieux et du patrimoine naturel et culturel qu'ils renferment ;

- Encourager la conservation de l'agrobiodiversité ${ }^{6}$ et de la biodiversité aquatique ;

- Servir de modèles de durabilité de sorte que l'on puisse en tirer des leçons pour d'autres applications.

\section{Caractéristiques marquantes}

Les aires protégées de la catégorie $\mathrm{V}$ résultent d'interactions biotiques, abiotiques et humaines et devraient présenter les caractéristiques essentielles suivantes :

- Paysage terrestre, côtier ou insulaire possédant des qualités pittoresques considérables ou particulières, avec la flore, la faune et les habitats importants associés et les caractéristiques culturelles qui y sont liées ;
- Une interaction équilibrée entre hommes et nature qui persiste depuis longtemps et qui a conservé son intégrité, ou pour laquelle on peut raisonnablement espérer qu'elle pourra restaurer cette intégrité ;

- Des formes uniques ou traditionnelles d'utilisation des sols, comme en témoignent p.ex. les systèmes agricoles et forestiers durables et les installations humaines qui ont évolué en équilibre avec leur paysage.

Les caractéristiques suivantes sont soubaitables:

- Possibilités d'activités récréatives et touristiques en harmonie avec le mode de vie et les activités économiques ;

- Organisations sociales uniques ou traditionnelles, comme en témoignent les coutumes, les modes de vie et les croyances locales ;

- Reconnaissance par des artistes en tous genres ainsi que dans les traditions culturelles (actuelles et anciennes) ;

- Possibilité d'une restauration écologique et/ou du paysage.

\section{Rôle dans le paysage terrestre ou marin}

Généralement, les aires protégées de la catégorie $\mathrm{V}$ jouent un rôle important dans la conservation à l'échelle des paysages terrestres ou marins, particulièrement en tant que pièces d'une mosaïque de formes de gestion, de désignations d'aires protégées et d'autres mécanismes de conservation :

- Certaines aires protégées de la catégorie V servent de tampons autour de la zone centrale d'une ou plusieurs aires plus strictement protégées, pour garantir que les activités d'utilisation des terres et de l'eau ne menacent pas leur intégrité ;

- Des aires protégées de la catégorie $\mathrm{V}$ peuvent aussi servir d'habitat reliant entre elles plusieurs autres aires protégées.

La catégorie $\mathrm{V}$ permet des contributions uniques à la conservation de la diversité biologique. En particulier :

- Des espèces ou des habitats qui ont évolué en association avec des systèmes de gestion culturaux et qui ne peuvent survivre que si ces systèmes de gestion sont maintenus ;

- Fournir un cadre lorsque les objectifs de la conservation doivent être respectés sur une grande étendue (p.ex. pour les prédateurs supérieurs) dans des paysages très peuplés qui ont toute une variété de formes de propriété, de modèles de gouvernance et d'utilisation des terres;

- De plus, les systèmes traditionnels de gestion sont souvent associés à des composantes importantes de l'agrobiodiversité ou de la biodiversité aquatique, qui ne peuvent être préservées qu'en maintenant ces systèmes. 


\section{Qu'est-ce qui fait que la catégorie $V$ est unique?}

\section{La catégorie $\mathbf{V}$ diffère des autres catégories des façons suivantes:}

\begin{tabular}{l|l} 
Catégorie & $\begin{array}{l}\text { L'intervention humaine est attendue. La } \\
\text { la }\end{array}$ \\
catégorie V ne donne pas la priorité à la \\
recherche même si elle peut offrir des \\
opportunités pour étudier les interactions \\
entre l'homme et la nature.
\end{tabular}

Catégorie Les aires protégées de la catégorie $\mathrm{V}$

lb ne sont pas de la « nature sauvage » comme la définit l'UICN. Nombre d'entre elles font l'objet d'interventions de gestion contraires au concept de la catégorie II.

Catégorie La catégorie II cherche à minimiser II l'activité humaine pour permettre un état « aussi naturel que possible ». La catégorie $\mathrm{V}$ inclut une option d'interaction permanente avec les hommes.

\section{Catégorie La catégorie III se focalise sur des} III caractéristiques spécifiques et singulières et insiste sur l'aspect monumental, unique et/ou rare d'éléments individuels, alors que celui-ci n'est pas requis pour les aires protégées de la catégorie $\mathrm{V}$ qui comprend plutôt des valeurs plus larges, multiples, à l'échelle du paysage.

Catégorie La catégorie $\mathrm{V}$ vise à protéger des IV paysages terrestres et marins généraux, qui ont une valeur pour la biodiversité alors que la catégorie IV vise souvent spécifiquement à protéger des espèces et des habitats cibles identifiés. Les aires protégées de la catégorie $\mathrm{V}$ sont souvent plus vastes que celles de la catégorie IV.

Catégorie La catégorie VI insiste sur le besoin de VI lier la conservation de la nature dans les aires naturelles à l'appui de modes de vie durables : inversement, la catégorie $\mathrm{V}$ met l'accent sur les valeurs résultant de longues interactions entre homme et nature, dans des conditions modifiées. Dans la catégorie $\mathrm{VI}$, l'accent est mis sur l'utilisation durable des produits et des services environnementaux (comme la chasse, le pâturage, la gestion des ressources naturelles) alors que dans la catégorie $\mathrm{V}$, il est mis sur des utilisations plus intensives (comme l'agriculture, la foresterie, le tourisme). La catégorie VI est d'habitude plus « naturelle » que la catégorie $\mathrm{V}$.

\section{Questions à considérer}

- Modèle relativement flexible, la catégorie V peut parfois offrir des options de conservation là où des aires protégées plus strictement ne sont pas réalisables;

- Les aires protégées de la catégorie $\mathrm{V}$ peuvent chercher à maintenir les pratiques en usage, à restaurer les systèmes de gestion anciens ou, peut-être plus souvent, à préserver les valeurs clés du paysage tout en adaptant le développement et les changements contemporains ; les décisions à ce sujet doivent être faites dans les plans de gestion ;

- L'accent mis sur les interactions entre l'homme et la nature avec le temps soulève la question conceptuelle pour toute aire protégée particulière de la catégorie $\mathrm{V}$ : sur quel point du continuum temporel la gestion doitelle se focaliser ? Et, dans une aire créée pour protéger les valeurs fondées sur des systèmes de gestion traditionnels, que se passe-t-il lorsque les traditions changent ou se perdent?

- Etant donné que les considérations sociales, économiques et de conservation font intégralement partie du concept de la catégorie V, il est important de définir des mesures de performances de toutes ces valeurs pour en mesurer la réussite.

- Comme les hommes sont les gestionnaires du paysage terrestre ou marins dans les aires protégées de la catégorie $\mathrm{V}$, il faut donner des lignes directrices claires pour savoir dans quelle mesure la prise de décisions peut être confiée aux résidents locaux et jusqu'où l'intérêt général doit prévaloir lorsqu'il y a conflit entre les besoins nationaux et locaux.

- Comment la catégorie V se distingue-t-elle d'une gestion durable dans le paysage environnant ? Comme une aire aux valeurs exceptionnelles ? Comme un exemple des meilleures pratiques de gestion ? L'approche de la catégorie $\mathrm{V}$ est peut-être celle qui se développe le plus vite de toutes les approches de gestion d'aires protégées.

- Il n'y a encore que peu d'exemples de l'application de la catégorie $\mathrm{V}$ à des milieux côtiers ou marins où une approche par " paysage marin protégé " pourrait être l'option de gestion la plus appropriée, et nous avons besoin de plus d'exemples (voir, p.ex. Holdaway, non daté). 


\section{Catégorie VI : Aire protégée avec utilisation durable des ressources naturelles}

Les aires protégées de la catégorie VI préservent des écosystèmes et des habitats, ainsi que les valeurs culturelles et les systèmes de gestion des ressources naturelles traditionnelles qui y sont associés. Elles sont généralement vastes, et la plus grande partie de leur superficie présente des conditions naturelles; une certaine proportion y est soumise à une gestion durable des ressources naturelles ; et une utilisation modérée des ressources naturelles, non industrielle et compatible avec la conservation de la nature, y est considérée comme l'un des objectifs principaux de l'aire.

Avant de choisir une catégorie, il faut d'abord vérifier que le site répond à la définition d'une aire protégée (page 10).

\section{Objectif premier}

- Protéger des écosystèmes naturels et utiliser les ressources naturelles de façon durable, lorsque conservation et utilisation durable peuvent être mutuellement bénéfiques.

\section{Autres objectifs}

- Encourager l'utilisation durable des ressources naturelles en prenant en compte les dimensions écologique, économique et sociale ;

- Quand c'est pertinent, encourager les bénéfices sociaux et économiques pour les communautés locales ;

- Faciliter la sécurité intergénérationnelle des moyens de subsistance des communautés locales - et donc s'assurer que de tels modes de vie sont durables;

- Intégrer d'autres approches culturelles, les systèmes de croyance et les visions du monde dans toute une gamme d'approches économiques et sociales de la conservation de la nature ;

- Contribuer au développement et/ou au maintien d'une relation plus équilibrée entre les hommes et le reste de la nature ;

- Contribuer au développement durable aux niveaux national, régional et local (dans ce dernier cas, principalement au bénéfice des communautés locales et/ou des populations autochtones qui dépendent des ressources naturelles protégées);

- Faciliter la recherche scientifique et le suivi environnemental, surtout en ce qui concerne la conservation et l'utilisation durable des ressources naturelles;
- Collaborer à la distribution de bénéfices aux gens, surtout aux communautés locales qui vivent dans ou à proximité de l'aire protégée classée ;

- Faciliter les loisirs et un tourisme modéré approprié.

\section{Caractéristiques marquantes}

- Dans les aires protégées de la catégorie VI, uniques dans le système des catégories de l'UICN, l'utilisation durable des ressources naturelles est considérée un moyen de conserver la nature, en synergie avec d'autres actions plus communes dans les autres catégories, telle la protection.

- Les aires protégées de la catégorie VI visent à conserver des écosystèmes et des habitats, de même que les valeurs culturelles et les systèmes de gestion des ressources naturelles qui leur sont associés. C'est pourquoi les aires protégées de cette catégorie tendent à être relativement vastes (même si ce n'est pas obligatoire).

- Cette catégorie n'est pas conçue pour intégrer les productions industrielles à grande échelle.

- En général, l'UICN recommande qu'une certaine proportion de l'aire soit maintenue dans des conditions naturelles, ${ }^{7}$ ce qui, dans certains cas, implique que celle-là soit définie comme une zone de non-prélèvement. Certains pays ont déjà fixé cette proportion aux deux-tiers ; l'UICN recommande que ces décisions se prennent au niveau national et parfois même au niveau de l'aire protégée elle-même.

\section{Rôle dans le paysage terrestre ou marin}

- Les aires protégées de la catégorie VI sont particulièrement adaptées aux approches par paysage.

- C'est une catégorie qui convient bien aux vastes aires naturelles, comme les forêts tropicales, les déserts et autres terres arides, aux systèmes de zones humides complexes, aux eaux côtières et à la haute mer, aux forêts boréales, etc. - non seulement parce qu'elle établit de grandes aires protégées, mais aussi parce qu'elle les relie à des groupes d'aires protégées, des corridors ou des réseaux écologiques.

- Les aires protégées de la catégorie VI peuvent aussi particulièrement convenir pour la conservation d'écosystèmes là où il y a peu, voire pas d'aires qui ne soient pas utilisées ou occupées et où ces utilisations et ces occupations sont des pratiques principalement traditionnelles avec peu d'impact, qui n'ont pas affecté substantiellement l'état naturel de l'écosystème.

Notons que cela n'exclut pas nécessairement une faible activité, comme la collecte de produits forestiers non ligneux. 


\section{Qu'est-ce qui fait que la catégorie VI est unique?}

L'attribution de la catégorie VI dépend des objectifs de gestion à long terme et aussi de caractéristiques locales spécifiques. Le tableau suivant expose certaines des raisons majeures de choisir la catégorie VI dans des situations spécifiques, plutôt que d'autres catégories.

\begin{tabular}{|c|c|}
\hline \multicolumn{2}{|c|}{$\begin{array}{l}\text { La catégorie VI diffère des autres catégories des } \\
\text { façons suivantes : }\end{array}$} \\
\hline $\begin{array}{l}\text { Catégorie } \\
\text { la }\end{array}$ & $\begin{array}{l}\text { Les aires protégées de la catégorie } \\
\text { VI conservent la biodiversité, } \\
\text { particulièrement à l'échelle de } \\
\text { l'écosystème et du paysage, mais le } \\
\text { but n'est pas de les protéger de toute } \\
\text { interférence humaine. Même si la } \\
\text { recherche scientifique y est importante, } \\
\text { elle ne sera considérée comme une } \\
\text { priorité que lorsqu'elle s'applique } \\
\text { aux formes d'utilisation durable des } \\
\text { ressources naturelles, que ce soit pour } \\
\text { les améliorer ou pour comprendre } \\
\text { comment minimiser les risques pour la } \\
\text { durabilité écologique. }\end{array}$ \\
\hline $\begin{array}{l}\text { Catégorie } \\
\mathrm{lb}\end{array}$ & $\begin{array}{l}\text { Les aires protégées de la catégorie } \\
\text { VI pourraient dans certains cas être } \\
\text { considérées comme proches de la « vie } \\
\text { sauvage », mais elles encouragent } \\
\text { explicitement l'utilisation durable, } \\
\text { contrairement à la situation dans les aires } \\
\text { de nature sauvage de la catégorie lb où } \\
\text { une telle utilisation devra rester minimale } \\
\text { et tout à fait secondaire par rapport aux } \\
\text { buts de la conservation. Elles contribuent } \\
\text { aussi au maintien des services } \\
\text { environnementaux, mais pas seulement } \\
\text { par la conservation exclusive de la nature } \\
\text { dans la mesure où l'utilisation durable } \\
\text { des ressources naturelles peut aussi } \\
\text { contribuer à la protection d'écosystèmes, } \\
\text { de vastes habitats et de processus } \\
\text { écologiques. }\end{array}$ \\
\hline $\begin{array}{l}\text { Catégorie } \\
\text { II }\end{array}$ & $\begin{array}{l}\text { Les aires protégées de la catégorie VI } \\
\text { visent à conserver des écosystèmes } \\
\text { aussi complets et fonctionnels que } \\
\text { possible, ainsi que leur diversité } \\
\text { d'espèces, leur diversité génétique } \\
\text { et les services environnementaux } \\
\text { associés, mais elles diffèrent des aires } \\
\text { de la catégorie Il par le rôle qu'elles } \\
\text { jouent dans la promotion de l'utilisation } \\
\text { durable des ressources naturelles. Le } \\
\text { tourisme peut être développé dans } \\
\text { les aires protégées de la catégorie } \\
\text { VI, mais seulement en tant qu'activité } \\
\text { très secondaire ou lorsqu'il fait partie } \\
\text { des stratégies socioéconomiques } \\
\text { des communautés locales (p.ex. } \\
\text { en lien avec le développement de } \\
\text { l'écotourisme). }\end{array}$ \\
\hline
\end{tabular}

\begin{tabular}{|c|c|}
\hline $\begin{array}{l}\text { Catégorie } \\
\text { III }\end{array}$ & $\begin{array}{l}\text { Les aires protégées de la catégorie VI } \\
\text { peuvent inclure la protection d'éléments } \\
\text { naturels ou culturels spécifiques, } \\
\text { y compris la diversité spécifique et } \\
\text { génétique, parmi leurs objectifs pour } \\
\text { autant que l'utilisation durable des } \\
\text { ressources naturelles fasse aussi } \\
\text { partie de ces objectifs ; cependant, } \\
\text { elles s'orientent davantage vers la } \\
\text { protection d'écosystèmes, de processus } \\
\text { écologiques et vers le maintien de } \\
\text { services écologiques par la protection } \\
\text { de la nature et la promotion d'approches } \\
\text { de gestion qui conduisent à l'utilisation } \\
\text { durable des ressources naturelles. }\end{array}$ \\
\hline $\begin{array}{l}\text { Catégorie } \\
\text { IV }\end{array}$ & $\begin{array}{l}\text { Les aires protégées de la catégorie VI } \\
\text { sont plus orientées vers la protection } \\
\text { des écosystèmes et des processus } \\
\text { écologiques et vers le maintien de } \\
\text { services environnementaux par la } \\
\text { protection de la nature et la promotion } \\
\text { de l'utilisation durable des ressources } \\
\text { naturelles. Alors que les aires protégées } \\
\text { de la catégorie IV ont tendance à prioriser } \\
\text { une gestion active, celles de la catégorie } \\
\text { VI encouragent l'utilisation durable des } \\
\text { ressources naturelles. }\end{array}$ \\
\hline $\begin{array}{l}\text { Catégorie } \\
\text { V }\end{array}$ & $\begin{array}{l}\text { La catégorie V s'applique à des aires } \\
\text { où les paysages ont été transformés } \\
\text { suite à de longues interactions avec les } \\
\text { hommes; les aires de la catégorie VI } \\
\text { restent des écosystèmes majoritairement } \\
\text { naturels. Dans la catégorie VI, l'emphase } \\
\text { est donc mise davantage sur la protection } \\
\text { d'écosystèmes naturels et de processus } \\
\text { écologiques, par la protection de la nature } \\
\text { et la promotion de l'utilisation durable des } \\
\text { ressources naturelles. }\end{array}$ \\
\hline
\end{tabular}

\section{Questions à considérer}

- La protection d'écosystèmes naturels et la promotion de l'utilisation durable doivent être intégrées et mutuellement bénéfiques ; la catégorie VI peut éventuellement montrer quelles sont les meilleures pratiques de gestion, qui pourraient être employées plus largement.

- Il faut que les autorités de gestion développent de nouvelles compétences et de nouveaux outils pour affronter les nouveaux défis qui sont issus de la planification, du suivi et de la gestion des aires où l'utilisation doit être durable.

- Il faut aussi développer de nouvelles formes appropriées de gouvernance qui conviennent aux aires protégées de la catégorie VI et aux multiples parties prenantes qui y sont souvent impliquées. La conservation à l'échelle du paysage inclut inévitablement un groupe diversifié de parties prenantes, ce qui exige des accords institutionnels méticuleux et des approches novatrices de la gouvernance. 


\section{Relations entre les catégories}

- Les catégories n'impliquent pas une hiérarchie simple en termes de qualité, d'importance ou de préservation du naturel

- Et les catégories ne sont pas nécessairement toutes égales en toute circonstance, mais devraient plutôt être choisies de façon à optimiser les opportunités et contrer les menaces sur la conservation

Les catégories n'impliquent pas une hiérarchie simple, ni en termes d'importance ou de qualité, ni pour quelque autre aspect que ce soit - par exemple, le degré d'intervention ou le caractère naturel. Mais toutes les catégories ne sont pas non plus égales, en ce sens qu'elles ne seront pas aussi utiles l'une que l'autre dans une situation donnée. Un des principes associés à la définition d'une aire protégée dit que : "Toutes les catégories contribuent à la conservation mais que les objectifs devraient être choisis en fonction des conditions particulières; toutes les catégories ne sont pas aussi utiles l'une que l'autre dans chaque situation".

Ceci implique qu'un système d'aires protégées bien équilibré peut envisager d'utiliser toutes les catégories même s'il peut arriver que toutes les options ne soient pas nécessaires ou pratiques dans chaque région ou dans chaque pays. Dans la grande majorité des cas, au moins une portion des aires protégées devrait relever des catégories plus strictement protégées, c.-à-d. I à IV. Le choix des catégories est souvent un exercice complexe qui devrait être guidé par les besoins et l'urgence de la conservation de la biodiversité, les possibilités de fournir des services écosystémiques, les besoins, les manques et les croyances des communautés humaines, les formes de propriété foncière, la rigueur de la gouvernance et les niveaux de population. Les décisions concernant les aires protégées font d'habitude l'objet de compromis, à la suite de processus consultatifs et d'utilisations foncières concurrentes. Il est important d'accorder aux objectifs de la conservation une attention et un poids adéquats dans les processus de prise de décision.

Les approches et les catégories de gestion ne sont pas nécessairement fixées définitivement, elles peuvent changer - et elles le font - si les circonstances se modifient ou si l'on se rend compte qu'un type d'approche ne marche pas. Toutefois, le changement de la catégorie d'une aire protégée doit faire l'objet de procédures qui sont au moins aussi rigoureuses que celles qui ont été appliquées lors de l'établissement de l'aire protégée et de sa catégorie.

De nombreuses personnes croient que les catégories impliquent une gradation du caractère naturel entre I et VI, mais la réalité est plus compliquée, comme le montre la Figure 1 ci-dessous, qui tente de comparer le caractère naturel de toutes les catégories.

Figure 1. Caractère naturel et catégories d'aires protégées de l'UICN

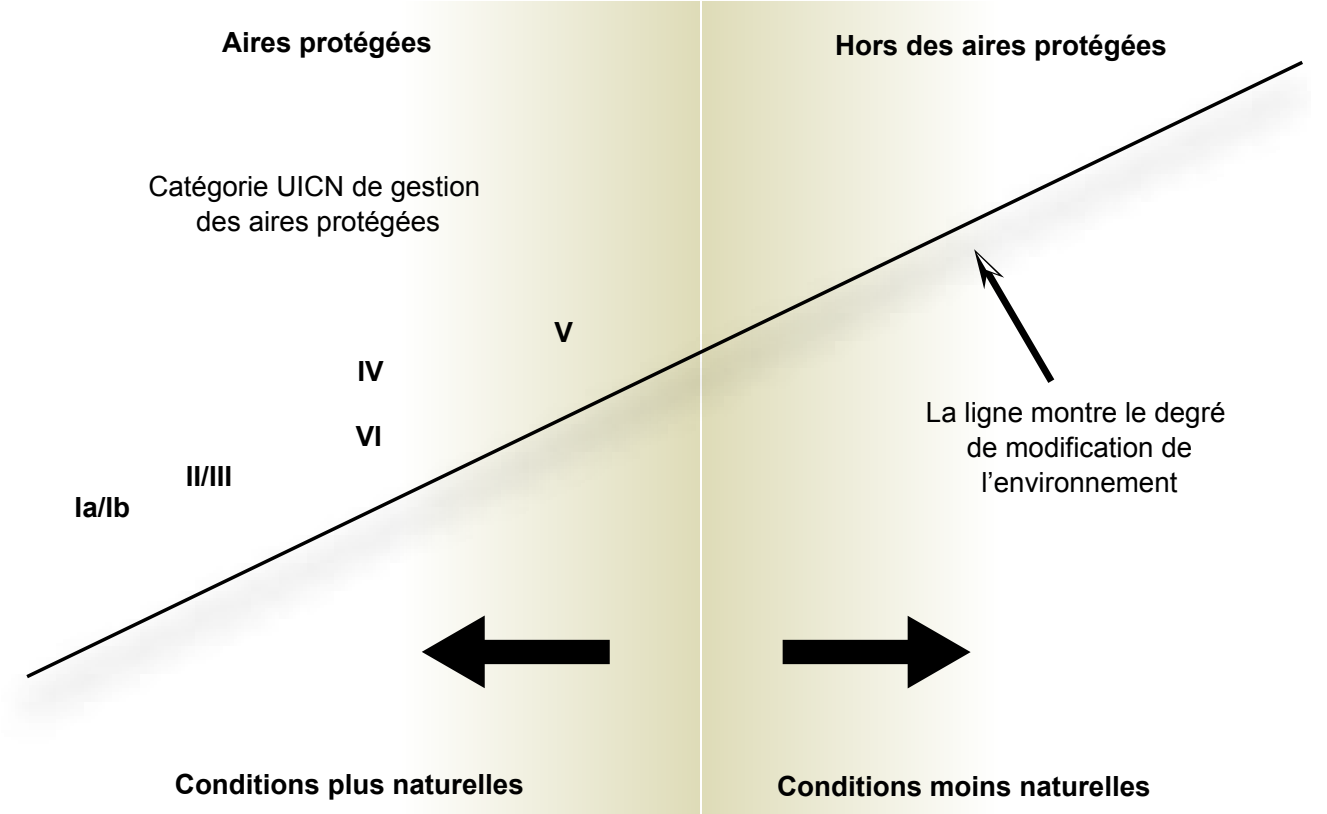





\section{Gouvernance}

Les catégories sont indépendantes de qui les possède, les contrôle ou a la responsabilité de leur gestion. Cependant, la gouvernance est aussi très importante. L'UICN a identifié différents types de gouvernance qui aident à comprendre, à planifier et à rendre compte des aires protégées. Cette section expose les types de gouvernance de l'UICN, explique comment ils sont liés aux catégories et examine comment une gouvernance par des populations autochtones, des communautés et des organismes privés peut contribuer aux systèmes d'aires protégées. 


\section{Gouvernance des aires protégées}

L'UICN reconnaît quatre grands types de gouvernance pour les aires protégées, chacun d'eux pouvant être associé à n'importe quel objectif de gestion

A. Gouvernance par le gouvernement

B. Gouvernance partagée

\section{Gouvernance privée}

D. Gouvernance par les populations autochtones et par les communautés locales

La définition d'une aire protégée et les catégories de gestion de l'UICN sont " neutres " quant aux types de propriété ou à l'autorité de gestion. En d'autres termes, la terre, l'eau et les ressources naturelles dans toute catégorie de gestion peuvent être la propriété et/ou sous la gestion directe d'organismes gouvernementaux, d'ONG, de communautés, de populations autochtones et de groupes privés - seuls ou en combinaison. Tant l'UICN que la CDB reconnaissent la légitimité de nombreux types de gouvernance. En ce qui concerne l'autorité qui détient le pouvoir de décision et la responsabilité de la gestion des aires protégées, l'UICN distingue quatre grands types de gouvernance des aires protégées.

Type A : Gouvernance par le gouvernement (au niveau fédéral, national, sous-national ou municipal). Un organisme gouvernemental (tel qu'un ministère ou une administration des Parcs relevant directement du Gouvernement) détient l'autorité et la responsabilité de la gestion de l'aire protégée, détermine ses objectifs de conservation (tels que ceux qui distinguent les catégories de l'UICN), développe et met en œuvre son plan de gestion et, souvent, est propriétaire des terres de l'aire protégée, de l'eau et des ressources associées. Des organes gouvernementaux sous-nationaux et municipaux peuvent aussi être en charge de ces fonctions et/ou être propriétaires des terres et des ressources des aires protégées. Dans certains cas, le gouvernement conserve le contrôle d'une aire protégée - en d'autres mots, il décide des objectifs de gestion de l'aire - mais en délègue la planification et/ou les tâches quotidiennes à une organisation parastatale, à une $\mathrm{ONG}$, à un opérateur privé ou à une communauté. Dans un cadre juridique et de gouvernance d'état, cela peut être, ou pas, une obligation légale d'informer ou de consulter les parties prenantes avant d'instaurer des aires protégées et de prendre ou d'appliquer des décisions concernant leur gestion. Mais les approches participatives sont de plus en plus fréquentes et elles sont généralement souhaitables. Les mesures de responsabilité varient aussi selon les pays.

Type B: Gouvernancepartagée. Des mécanismes et des processus institutionnels complexes sont utilisés pour partager l'autorité et les responsabilités de la gestion entre une pluralité d'acteurs gouvernementaux et non gouvernementaux (formellement et informellement) habilités. La gouvernance partagée, appelée aussi cogouvernance, se présente sous diverses formes. Dans la gestion " collaborative ", l'autorité décisionnelle et la responsabilité sont confiées à un organisme, mais celui-ci est tenu, par la loi ou par décision politique, d'informer ou de consulter les autres parties prenantes. La participation à la gestion collaborative peut être renforcée en confiant à des organes composés de plusieurs parties prenantes la responsabilité de développer des propositions techniques pour la réglementation et la gestion de l'aire protégée, qui seront ensuite soumises à l'approbation finale de l'autorité décisionnelle. Dans une gestion « conjointe », divers acteurs siègent dans un organe de gestion qui possède l'autorité et la responsabilité décisionnelles. Les décisions peuvent, ou pas, exiger un consensus. Dans chacun de ces cas, une fois que les décisions concernant la gestion sont prises, leur mise en œuvre doit être déléguée à des organismes ou à des particuliers agréés. Une forme particulière de gouvernance partagée a trait aux aires protégées transfrontalières, qui impliquent au moins deux gouvernements et parfois d'autres intervenants locaux.

Type C: Gouvernance privée. La gouvernance privée comprend les aires protégées inscrites dans des modèles de contrôle et/ou de propriété assurés par un particulier, une coopérative, une ONG ou une société, et qui sont gérées à des fins lucratives ou non. Les aires acquises par des ONG explicitement pour la conservation en sont des exemples typiques. De nombreux propriétaires particuliers pratiquent aussi la conservation par respect pour la terre et par souci de préserver des valeurs esthétiques et écologiques. Des mesures d'incitation, comme des profits tirés de l'écotourisme et de la chasse ou une réduction d'impôts et de taxes, encouragent souvent ce type de gouvernance. Dans tous ces cas, l'autorité sur la gestion de l'aire protégée et de ses ressources revient aux propriétaires qui déterminent l'objectif de la conservation, développent et mettent en ouvre des plans de gestion et restent maîtres des décisions, pour autant qu'elles respectent la législation concernée. Quand le gouvernement n'a manifesté aucune reconnaissance officielle, la responsabilité des aires protégées privées envers la société peut être limitée. Il est possible de négocier un certain degré de responsabilité avec le gouvernement, par exemple en termes de sécurité à long terme, en échange d'incitants spécifiques (comme dans le cas des Servitudes de conservation ou des Fiducies foncières).

Type D : Gouvernance par des populations autochtones et des communautés locales. Ce type inclut deux sous-ensembles principaux : (1) les aires et les territoires des populations autochtones établis et gérés par des populations autochtones et (2) les zones de conservation communautaire, créées et gérées par les communautés. Ces sous-ensembles, qui peuvent ne pas être nettement distincts, s'appliquent tant à des populations qu'à des communautés sédentaires et nomades. L'UICN définit ce type de gouvernance comme des aires protégées où l'autorité et la responsabilité de la gestion sont confiées aux peuples autochtones et aux communautés locales sous diverses formes d'institutions ou de réglementations coutumières ou juridiques, formelles ou informelles. Celles-ci 
peuvent être relativement complexes. Par exemple, les ressources de la terre ou de la mer peuvent être une propriété collective gérée collectivement, alors que d'autres ressources peuvent être gérées par des particuliers ou sur une base clanique. Différents peuples ou communautés autochtones peuvent être responsables de la même aire à différentes périodes, ou de différentes ressources dans la même aire. Les réglementations s'entremêlent généralement avec des valeurs culturelles et spirituelles. Le droit coutumier et les organisations qui gèrent les ressources naturelles ne bénéficient pas souvent de la reconnaissance statutaire légale ou d'un pouvoir disciplinaire. Dans d'autres cas, cependant, les peuples autochtones et/ou les communautés locales sont pleinement reconnues comme les autorités en charge d'aires protégées reconnues par l'état ou possèdent un droit légal sur la terre, l'eau ou les ressources. Quelle que soit la structure, les accords de gouvernance exigent que l'aire qui est sous le contrôle des peuples autochtones et/ou des communautés locales ait des institutions et des réglementations identifiables responsables de l'atteinte des objectifs de l'aire protégée.
Les quatre types de gouvernance décrits ci-dessus sont intégrés dans les catégories de gestion dans la matrice suivante (adapté de Borrini-Feyerabend et al. 2004).

Notons que les types de gouvernance décrivent les différents types d'autorité et de responsabilité de gestion qui peuvent exister pour les aires protégées, mais qui ne sont pas forcément liés à la propriété. Dans certains types de gouvernance, p.ex. les aires protégées d'état ou privées, la gouvernance et la propriété relèvent de la même entité. Mais dans certains cas, ceci dépend de la législation du pays : par exemple, de nombreuses aires protégées par les populations autochtones et d'aires conservées par des communautés se situent sur des terres d'état. Dans des aires protégées vastes et complexes, particulièrement dans les catégories $\mathrm{V}$ et VI, il peut y avoir différents types de gouvernance au sein des limites d'une même aire protégée, éventuellement sous la responsabilité d'une autorité générale. Dans le cas de la plupart des aires marines protégées, le propriétaire peut être l'Etat, qui peut gérer directement seul ou déléguer la

Tableau 3. "La matrice UICN des aires protégées " : un système de classification des aires protégées comprenant la catégorie de gestion et le type de gouvernance

\begin{tabular}{|c|c|c|c|c|c|c|c|c|c|c|c|}
\hline \multirow{2}{*}{$\begin{array}{l}\text { Types de } \\
\text { gouvernance } \\
\text { Catégories d'aires } \\
\text { protégées }\end{array}$} & \multicolumn{3}{|c|}{$\begin{array}{l}\text { A. Gouvernance } \\
\text { par gouvernement }\end{array}$} & \multicolumn{3}{|c|}{$\begin{array}{l}\text { B. Gouvernance } \\
\text { partagée }\end{array}$} & \multicolumn{3}{|c|}{$\begin{array}{l}\text { C. Gouvernance } \\
\text { privée }\end{array}$} & \multicolumn{2}{|c|}{$\begin{array}{l}\text { D. Gouvernance par } \\
\text { peuples autochtones } \\
\text { et communautés } \\
\text { locales }\end{array}$} \\
\hline & 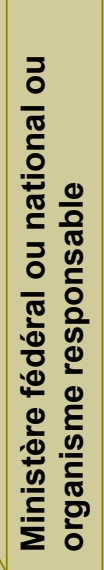 & 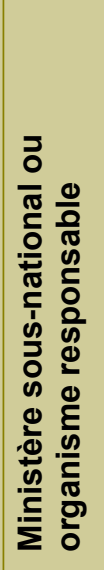 & 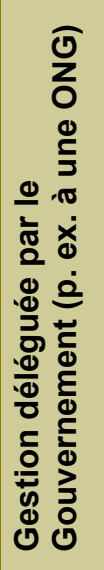 & 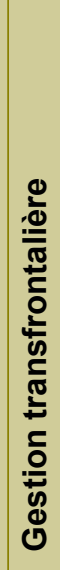 & 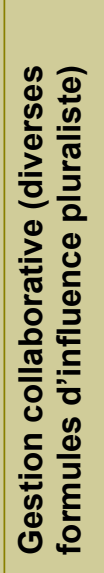 & 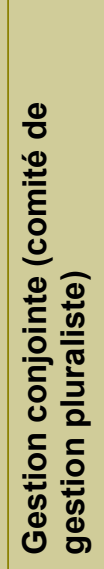 & 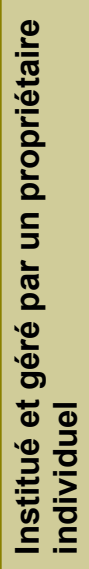 & 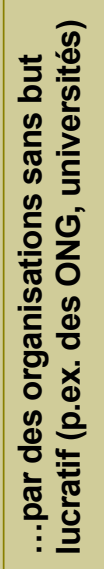 & 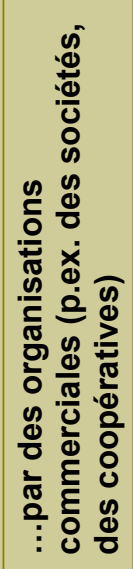 & 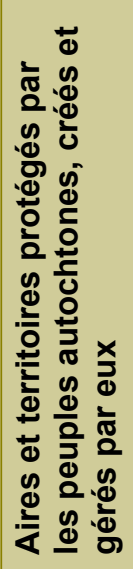 & 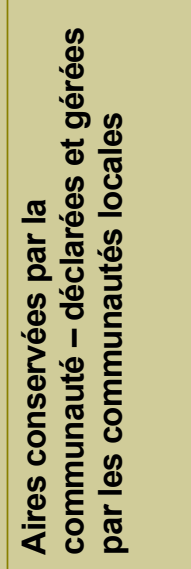 \\
\hline \multicolumn{12}{|l|}{$\begin{array}{l}\text { la. Réserve naturelle } \\
\text { intégrale }\end{array}$} \\
\hline \multicolumn{12}{|l|}{$\begin{array}{l}\text { Ib. Zone de nature } \\
\text { sauvage }\end{array}$} \\
\hline \multicolumn{12}{|l|}{ II. Parc national } \\
\hline \multicolumn{12}{|l|}{ III. Monument naturel } \\
\hline \multicolumn{12}{|l|}{$\begin{array}{l}\text { IV. Aire de gestion des } \\
\text { habitats ou des espèces }\end{array}$} \\
\hline \multicolumn{12}{|l|}{$\begin{array}{l}\text { V. Paysage terrestre ou } \\
\text { marin protégé }\end{array}$} \\
\hline $\begin{array}{l}\text { VI. Aire protégée avec } \\
\text { utilisation durable des } \\
\text { ressources naturelles }\end{array}$ & & & & & & & & & & & \\
\hline
\end{tabular}


gestion aux communautés, à des ONG ou à d'autres. Il existe pourtant de nombreuses aires marines protégées où le droit coutumier des populations autochtones est reconnu et respecté par l'ensemble de la société. Dans les eaux internationales et dans l'Antarctique, où il n'existe pas d'autorité étatique unique, les aires protégées requièrent inévitablement un type de gouvernance partagée.

\section{Enregistrement des types de gouvernance}

L'UICN suggère que le type de gouvernance d'une aire protégée soit enregistré en même temps que son objectif de gestion (catégorie) dans les systèmes nationaux de statistiques et de responsabilité environnementales et dans les bases de données sur les aires protégées. Dans certains cas, décider du type de gouvernance peut être aussi délicat et complexe, sinon plus, qu'identifier la catégorie, et l'un peut orienter et influencer l'autre; de plus, de nombreuses aires protégées sont susceptibles de changer de type de gouvernance avec le temps. Comme il a déjà été mentionné, dans le cas de grandes aires protégées, plusieurs types de gouvernance peuvent coexister au sein des limites d'une même aire protégée.

En envisageant la gouvernance du point de vue des rapports vers la Base de données mondiale sur les aires protégées, la CMAP/UICN propose d'adopter une structure en deux dimensions. Même si les objectifs de gestion correspondant aux catégories peuvent être développés et assignés sans tenir compte de la gouvernance, des comparaisons entre des aires protégées et leur efficacité seront considérablement renforcées en notant aussi bien le type de gouvernance que la catégorie de gestion dans les bases de données futures. Les catégories d'aires protégées ne sont pas taxonomiques, contrairement aux types de gouvernance ; cependant une classification en deux dimensions permet de classer facilement les objectifs de gestion (ex. catégorie I-VI) et le type de gouvernance (ex. A-D, comme décrit plus haut). En reprenant les lettres de désignation utilisées plus haut, par exemple, le Parc National de Yellowstone (USA) pourrait être décrit comme catégorie II-A ; le Mornington Wildlife Sanctuary serait ainsi II-C ; le Parc National de Snowdonia (GB) serait $\mathrm{V}$-B ; et Coron Island (Philippines) serait une combinaison de I-D et de V-D.

\section{Qualité de la gouvernance}

Pour des aires protégées de toutes les catégories de gestion, l'efficacité de gestion fournit une mesure de l'atteinte réelle des objectifs de la conservation. L'efficacité de gestion est aussi influencée par la qualité de la gouvernance, c'est-à-dire qu'elle nous dit comment un régime de gouvernance fonctionne. En d'autres mots, le concept de qualité de la gouvernance appliqué à toute situation spécifique tente d'apporter la réponse à des questions telles que : "Ceci est-il de la bonne gouvernance ?» et " Ce modèle de gouvernance peut-il être amélioré pour produire des bénéfices aussi bien pour la conservation que pour les moyens de subsistance?»
"La bonne gouvernance d'une aire protégée " peut se comprendre comme un système de gouvernance qui répond aux principes et valeurs librement choisis par les populations et le pays concernés et consacrés dans leur constitution, leurs lois sur les ressources naturelles, la législation et la politique en matière d'aires protégées, et les pratiques culturelles et les lois coutumières. Tous ceux-ci devraient refléter les principes reconnus au niveau international pour la bonne gouvernance (ex. Graham et al. 2003). Des accords et des instruments internationaux ont établi des principes de gouvernance et des valeurs, comme la CDB, la Convention d'Aarhus, la Convention des NU sur la lutte contre la désertification, la Déclaration universelle des droits de l'homme et la Déclaration des NU sur les droits des peuples autochtones. Un certain nombre de processus internationaux et régionaux ont aussi été essentiels dans l'établissement de ce calendrier, comme le Congrès mondial des parcs, en Afrique du Sud, en 2003, le Premier Congrès mondial des aires marines protégées en Australie, en 2005, et le Deuxième Congrès d'Amérique latine sur les parcs nationaux et autres aires protégées, en Argentine, en 2007. En se basant sur tous ceux-ci et sur l'expérience de terrain, l'UICN a exploré une série de grands principes de bonne gouvernance des aires protégées, y compris :

- Légitimité et droit à la parole - dialogue social et accords collectifs sur les objectifs et les stratégies de gestion des aires protégées sur la base de la liberté d'association et de parole, sans discrimination liée au sexe, à l'ethnie, au mode de vie, aux valeurs culturelles ou à quelque autre caractéristique ;

- Subsidiarité - attribuer l'autorité et la responsabilité de la gestion aux institutions qui sont les plus proches des ressources en jeu ;

- Impartialité - partage équitable des coûts et bénéfices de la création et de la gestion des aires protégées et possibilité de recourir à un jugement impartial en cas de conflit ;

- Pas de préjudice - s'assurer que les coûts de la création et de la gestion des aires protégées ne génèrent pas, ou n'aggravent pas la pauvreté ou la vulnérabilité ;

- Direction - encourager et préserver une vision créative et cohérente à long terme pour l'aire protégée et pour ses objectifs de conservation ;

- Performance - conserver efficacement la biodiversité tout en répondant aux préoccupations des parties prenantes et faire une utilisation raisonnable des ressources ;

- Responsabilité - faire une distinction nette entre les devoirs de chacun et garantir que les parties prenantes rapportent de façon adéquate et assument correctement leurs responsabilités;

- Transparence - garantir que toutes les informations pertinentes sont accessibles à toutes les parties prenantes;

- Droits humains - respecter les droits humains, dans le contexte de la gouvernance des aires protégées, y compris ceux des générations futures. 
La gouvernance des populations autochtones et des communautés locales, ainsi que la gouvernance privée, sont discutées en détail plus bas.

\section{Gouvernance par les populations autochtones et les communautés locales}

Une note sur la terminologie : les concepts de gouvernance par les peuples autochtones et les communautés locales évoluent encore et different selon les régions du monde. Certains peuples autochtones voudraient voir leurs territoires clairement distingués de ceux des communautés locales. Dans d'autres cas, populations autochtones et communautés locales cohabitent et gèrent ensemble les aires et même, dans d'autres cas encore, les populations autochtones emploient elles-mêmes les termes « aires de conservation communautaire " pour des raisons pratiques, par exemple, là où le terme " autochtone " n'est pas reconnu. Il existe aussi des différences régionales concernant le terme "territoire ". Parmi les peuples autochtones et les communautés locales, il $\mathrm{y}$ a des cas où ce sont les termes " aire de conservation " qui sont utilisés et d'autres où " aire protégée » est préféré : nous utilisons ici toute une gamme de termes. Nous résumons les concepts ci-dessous et nous donnons une description des territoires et des aires protégées des populations autochtones.

Même si certaines des aires protégées gérées par des peuples autochtones et des communautés locales existent depuis des centaines, voire des milliers d'années, leur reconnaissance par les gouvernements nationaux et leur intégration dans les systèmes nationaux d'aires protégées sont des événements beaucoup plus récents qui méritent une attention particulière. Les aires protégées par des peuples autochtones, les territoires préservés par les peuples autochtones et les aires de conservation communautaire (que nous résumons sous le sigle APAC Aires du patrimoine autochtone et communautaire [APAC est l'équivalent de l'abréviation anglaise ICCA (Indigenous and community conserved areas) - Note du traducteur] ont trois caractéristiques essentielles :

- Les populations autochtones et/ou communautés locales sont extrêmement concernées par les écosystèmes en question - elles y sont d'habitude liées pour des raisons culturelles (p. ex. en raison de leur valeur comme sites sacrés) et/ou parce qu'ils sont à la base de leurs moyens de subsistance, et/ou parce que ce sont leurs territoires traditionnels de par le droit coutumier.

- Ces populations autochtones et/ou communautés locales sont les acteurs principaux (elles "gardent la main ») dans la prise de décisions et la mise en ouvre des décisions concernant la gestion de l'écosystème concerné, ce qui implique qu'elles possèdent une institution qui exerce l'autorité et la responsabilité et qu'elles sont capables d'appliquer des réglementations.
- Les décisions et les efforts de gestion des populations autochtones et/ou des communautés locales mènent et contribuent à la conservation des habitats, des espèces, des fonctions écologiques et des valeurs culturelles associées, même si l'intention originale portait peut-être sur une variété d'objectifs qui n'étaient pas directement liés à la protection de la biodiversité.

Il est de plus en plus évident que les APAC qui correspondent à la définition et aux normes des aires protégées peuvent assurer une réelle conservation de la biodiversité qui répond à n'importe quel objectif de gestion des catégories de l'UICN, particulièrement à des endroits où il est politiquement ou socialement impossible d'instaurer des aires protégées gérées par le gouvernement, ou là où elles risqueraient d'être mal gérées. Les APAC commencent à être reconnues comme des parties de stratégies de planification de la conservation, qui peuvent compléter les aires protégées gérées par les gouvernements, les aires protégées privées et diverses formes de gouvernance partagée (voir http://www.APACforum.org/). Mais elles sont encore davantage l'exception que la règle.

Aujourd'hui, la plupart des APAC ne sont pas encore officiellement reconnues, protégées ni même considérées comme des composantes de systèmes d'aires protégées nationaux. Dans certains cas, il peut y avoir de bonnes raisons pour que ce soit ainsi - y compris la réticence des populations autochtones et/ou des communautés locales concernées à devenir mieux connues, et plus dérangées, par exemple quand le site a un caractère sacré qui requiert la discrétion ou lorsque les populations autochtones en question choisissent de gérer leur terre uniquement selon le droit coutumier. Alors que les nations évoluent vers la reconnaissance des APAC, ces sensibilités doivent être respectées. Selon la situation spécifique et les principales préoccupations des populations autochtones et/ou des communautés locales concernées, la réponse appropriée du gouvernement peut varier de l'intégration des APAC dans le système national d'aires protégées à une reconnaissance " en dehors du système ", voire à aucune reconnaissance formelle quelle qu' elle soit. Cette dernière option, évidemment, doit être choisie lorsque la reconnaissance formelle pourrait ébranler ou perturber les APAC en question.

La plupart des APAC font face à de formidables forces de changement qu' elles seraient peut-être mieux capables d'affronter avec l'aide que peut représenter une reconnaissance ou une approbation officielles, spécialement lorsque l'alternative la plus probable risque d'être l'exploitation, p.ex. pour le bois ou le tourisme. Dans ces cas-là, si les APAC répondent à la définition et aux normes des aires protégées, la reconnaissance au sein d'un système national d'aires protégées, ou d'autres modes de reconnaissance officielle, peuvent offrir aux populations autochtones et aux communautés locales des garanties supplémentaires pour leur terre. Ceci doit cependant être assorti de la reconnaissance par l'Etat du fait que les APAC sont, par nature, différentes des 
aires protégées gérées par l'Etat - particulièrement en ce qui concerne les institutions de gestion. Il faut toutefois noter que la reconnaissance formelle des APAC peut entraîner de nouveaux dangers, comme un accroissement du nombre de visiteurs et de l'intérêt commercial pour le site ou encore plus d'interférence du gouvernement. Les populations autochtones et les communautés locales craignent aussi que la reconnaissance officielle des APAC ne les propulse dans des systèmes plus vastes sur lesquels ils n'auront, fondamentalement, aucun contrôle.

Bien que l'on reconnaisse de plus en plus le rôle positif que les APAC peuvent jouer dans la conservation de la biodiversité, la communauté internationale s'inquiète aussi de la possibilité que des APAC «faibles » s'ajoutent à des systèmes nationaux d'aires protégées comme une alternative moins coûteuse et plus avantageuse d'un point de vue politique que d'autres options de conservation. Certains s'inquiètent aussi du fait que, comme les sociétés évoluent, les approches communautaires de la gestion risquent de changer aussi et que certaines des valeurs et des comportements traditionnels qui ont aidé à conserver la biodiversité disparaissent dans le même mouvement. Les APAC formelles qui ne sont pas à même de préserver leurs pratiques traditionnelles de conservation sont pires que des APAC informelles, non reconnues.

Enfin, tout en gardant à l'esprit tous les avertissements mentionnés ci-dessus, la reconnaissance des APAC qui répondent parfaitement à la définition et aux normes des aires protégées dans les stratégies nationales et régionales en matière d'aires protégées est un des développements actuels les plus importants de la conservation. Quelques réflexions initiales sur les critères exigibles pour cette reconnaissance ont déjà été publiées (BorriniFeyerabend et al. 2004), et de nouveaux développements sont attendus dans le cadre de la Série de la CMAP/UICN «Lignes directrices sur les meilleures pratiques pour les aires protégées ».

\section{Territoires et aires protégées des populations autochtones}

De nombreuses aires protégées officiellement désignées sont en même temps les terres et les eaux ancestrales de populations, de cultures et de communautés autochtones, spécialement dans des régions comme l'Amérique latine, l'Amérique du Nord, l'Océanie, l'Afrique, l'Asie et l'Arctique. L'UICN a depuis longtemps adopté et encouragé, en matière d'aires protégées, les politiques qui respectent les droits et les intérêts des populations autochtones et elle a développé des outils et des approches pour faciliter leur reconnaissance et leur mise en œuvre.

Dans le respect de ses politiques, I'UICN applique les principes de bonne gouvernance suivants, dans la mesure où ils concernent des aires protégées qui se superposent à des terres, des eaux et des ressources traditionnelles des populations autochtones :

- Les aires protégées établies sur des terres, des territoires et des ressources autochtones doivent respecter les droits de leurs propriétaires, gardiens ou utilisateurs traditionnels ;

- La gestion des aires protégées doit aussi respecter les institutions et le droit coutumier des populations autochtones ;

- C'est pourquoi les aires protégées doivent reconnaître les propriétaires ou les gardiens autochtones comme les détenteurs des pouvoirs statutaires dans leurs aires, et donc respecter et appuyer les populations autochtones qui exercent l'autorité et le contrôle sur de telles aires.

Ces dernières années, il y a eu d'importants développements concernant les aires protégées chevauchant des terres, des eaux et des ressources de populations autochtones. Tout d'abord, l'UICN, lors de ses Congrès mondiaux de la nature, a adopté des politiques spécifiques pour les aires protégées et les droits des populations autochtones. Ensuite, au niveau national, de nombreux pays ont adopté et appliquent de nouveaux cadres juridiques et politiques concernant les droits des populations autochtones, qui ont d'importantes implications pour les aires protégées. Enfin, au niveau international, plusieurs instruments comme le Programme de travail sur les aires protégées de la CDB ou la Déclaration des NU sur les droits des populations autochtones ont été adoptés et ont changé significativement le paysage politique en ce qui concerne les populations autochtones et les aires protégées.

Suite à ces évolutions politiques, d'importants changements ont eu lieu aussi sur le terrain. De nombreuses aires protégées d'état qui chevauchent des terres, des eaux et des ressources de populations autochtones ont intégré des accords de gouvernance partagée et ont évolué vers une autogestion par les populations autochtones. Dans des pays comme le Canada, l'Australie, la Nouvelle-Zélande et plusieurs pays d'Amérique latine, de nombreuses aires protégées nouvelles ont été créées à la demande ou à l'initiative de propriétaires autochtones, ou par des arrangements conjoints avec les gouvernements. Dans ces cas-là, les droits autochtones sur les terres et les ressources, de même que la gouvernance autochtone du terroir, sont des caractéristiques clés.

De nombreuses populations autochtones voient les aires protégées comme des outils qui leur sont très utiles puisqu'elles peuvent renforcer la protection de leurs territoires, de leurs terres et de leurs ressources contre des menaces extérieures, proposer de nouvelles opportunités d'utilisation durable, renforcer la protection d'endroits critiques au nom de la culture et consolider les institutions autochtones d'aménagement du territoire. Dans ces conditions, les aires protégées des peuples autochtones sont un phénomène croissant et important et qui va probablement augmenter encore dans le monde entier. 
Tous les territoires, les terres et les ressources autochtones ne se conforment pas totalement à la définition d'une aire protégée mais certains le font et peuvent être considérés comme des " aires protégées ». Par conséquent, les aires protégées des peuples autochtones peuvent être définies comme :

« des espaces géographiques clairement définis, dans des espaces terrestres et aquatiques occupés et utilisés de manière traditionnelle par une population autochtone, une nation ou une communauté donnée, qui sont volontairement dédiés et gérés, par des moyens efficaces, juridiques ou autres, y compris leurs lois et institutions coutumières, pour assurer la conservation à long terme de la nature et des services écosystémiques associés, ainsi que la protection des communautés résidentes, de leur culture, de leur moyens de subsistance et de leurs créations culturelles ».

Les principales caractéristiques marquantes des aires protégées des peuples autochtones concernent les accords politiques passés entre ceux-ci et les autorités nationales pour l'administration des terres des peuples autochtones et de leurs ressources. Ces caractéristiques sont principalement les suivantes :

1. Elles se fondent sur les droits collectifs de la population autochtone, de la nation ou de la communauté concernée sur les terres, les territoires et les ressources, dans un contexte national ;

2. Elles sont établies comme aires protégées en application du droit d'autodétermination, qui s'exerce principalement par :

- L'auto-proclamation de l'aire protégée par le peuple autochtone ou la nation qui a des droits territoriaux collectifs sur l'aire ;

- Le consentement libre, préalable et informé du peuple, de la nation ou de la communauté qui a des droits territoriaux sur l'aire, au cas où la proposition de désignation vient d'organes du gouvernement, d'organisations de conservation et d'autres acteurs.

3. Elles se fondent sur une occupation ancestrale ou traditionnelle ;

4. L'occupation, l'utilisation et la gestion sont liées à, et dépendent de la structure plus large, socioculturelle et politique, d'un peuple ou d'une nation, qui inclut leur droit et leurs institutions coutumiers ;

5. Elles sont autogérées par des institutions autochtones au sein de leurs territoires et des aires protégées qui s'y trouvent, en application des accords passés avec les autorités responsables du réseau des aires protégées.

L'UICN reconnait qu'il devrait exister des orientations spécifiques relatives au large thème des territoires des populations autochtones et de leurs aires protégées et elle espère travailler avec les organisations représentantes des populations autochtones du monde entier pour y arriver.

\section{Etapes possibles pour déterminer si le territoire d'un peuple autochtone ou une APAC est une " aire protégée " et pour le reconnaître au sein d'un système d'aires protégées}

- Déterminer si l'aire et sa gouvernance actuelle correspondent à la définition d'une aire protégée par l'UICN.

- Déterminer si l'aire répond aussi aux critères d'une aire protégée dans la législation et la politique nationales.

- Si oui, déterminer si elle s'intègre dans la typologie existante des catégories d'aires protégées du pays concerné. L'aire peut-elle remplir les conditions pour être appelée parc national, sanctuaire, réserve de faune ou toute autre catégorie existante ? Point important, cette catégorie permet-elle au système d'auto-gouvernance de la communauté de se poursuivre ? Autorise-t-elle des objectifs de gestion qui peuvent être, conceptuellement et/ou pratiquement, différents de la conservation per se?

- Lorsque la législation et les politiques nationales sont totalement compatibles avec les pratiques locales, les agences de conservation devraient accepter, ou reconnaître formellement, que l'autorité et les pouvoirs décisionnels pour l'établissement et la gestion de l'aire devraient être du ressort des peuples autochtones et/ou des communautés locales concernées. C'est une démarche importante qui leur permettra directement d'appliquer leurs décisions (comme dans le cas où une ordonnance pour le contrôle de la pêche peut fournir l'appui légal nécessaire à un sanctuaire marin reconnu comme communautaire).

- Lorsqu'il y a incompatibilité entre la gouvernance des peuples autochtones ou de la communauté dans une aire importante et les lois et réglementations nationales sur les aires protégées, il peut être nécessaire d'apporter des ajustements juridiques et politiques aux conditions statutaires actuelles, de sorte que les peuples autochtones et les communautés locales concernées puissent conserver leur système de gouvernance. Souvent, ce que les peuples autochtones ou 
les communautés locales demandent, c'est une garantie de droit foncier coutumier, de droits d'utilisation et d'accès qui soit sanctionnée par une démarcation des territoires et des ressources. Pour que cela se fasse, toutefois, il peut être nécessaire que l'institution gouvernant l'aire soit reconnue comme une organisation légale. Comme ceci peut affecter la façon dont les peuples autochtones et les communautés locales s'organisent et gèrent leurs aires et territoires, il est important qu'elles statuent sur ces questions.

- Quand les incompatibilités sont levées, l'agence peut entamer un processus de négociations qui peut se clôturer par un arrangement contractuel entre les peuples autochtones et/ou les communautés locales concernées et les autorités nationales ou sous-nationales. Un tel arrangement contractuel pourrait, par exemple, reconnaître l'aire et lui accorder une certaine protection légale ou technique et un soutien financier, y compris en l'incluant comme une portion autonome dans le système national d'aires protégées. Dans d'autres cas, elle peut transformer l'aire en une aire protégée dotée d'une gouvernance partagée.

- Quand les peuples autochtones et/ou les communautés locales concernées et les autorités nationales ou sous-nationales ont trouvé un accord pour reconnaître l'aire comme aire protégée, les règles et réglementations pertinentes doivent être clarifiées et rendues publiques. Ceci peut signifier le simple enregistrement des règles coutumières existantes, sans qu'interfèrent les organismes d'état, ou l'incorporation de nouveaux conseils, méthodes et outils dans ces règles. Les règles doivent spécifier quelle sorte de zonage des terres et des ressources existe, quels droits communautaires et individuels existent (y compris des droits de propriété), quelles structures institutionnelles gèrent l'aire, si la récolte durable des ressources est autorisée et, si oui, comment (p.ex. avec des limites sur les quantités, les espèces et les saisons), et quels processus il faut suivre pour " dés-homologuer » l'aire si les objectifs de conservation acceptés ne sont pas atteints. II peut aussi être utile de clarifier et de rendre compte de la subdivision des droits et des responsabilités entre les peuples autochtones et les communautés locales concernées elles-mêmes et de préciser les dispositions en cas de mésusage des droits et du pouvoir de la part des autorités à tout niveau.

- Parmi les processus de gouvernance, les limites doivent être réellement respectées et protégées contre toute menace extérieure. Quelles sortes de mécanismes coutumier et local de surveillance et de maintien de la loi sont reconnues par l'Etat? Par exemple, les membres des populations autochtones et des communautés locales peuvent-ils appréhender des contrevenants ? L'aide du gouvernement est-elle nécessaire ? Qui est juge en cas de controverse ? Qui est responsable des campagnes d'information nécessaires pour que le public respecte les APAC et les aires protégées autochtones ? Les réponses à ces questions sont importantes pour que ces aires restent des aires protégées efficaces dans le temps.

\section{Gouvernance privée}

Les aires protégées privées sont un sous-ensemble vaste et encore croissant des aires protégées du monde entier, qui ont des représentantes dans toutes les catégories de l'UICN, mais elles sont jusqu'à présent sous-représentées dans l'ensemble des aires reconnues par l'UICN et signalées dans la BDMAP.

Les aires protégées privées ne sont généralement pas sous l'autorité directe du gouvernement. Il y a trois entités en charge des aires protégées privées, chacune impliquant une gestion particulière :

- Individuelle (l'aire est sous le contrôle d'une seule personne ou d'une famille).

- Organisation non gouvernementale (l'aire est sous le contrôle d'une organisation à but non lucratif qui œuvre pour remplir une mission spécifique et qui est d'habitude contrôlée par une direction, un conseil et des membres donateurs). Dans de rares cas, celles-ci peuvent inclure des coopératives (p.ex. la Comunidad Ahuenco, au Chili).

- Appartenant à une société (l'aire est sous le contrôle d'une société privée, commerciale, ou d'un groupe de gens habilités à agir comme une entité unique, d'habitude contrôlés par une direction, un conseil de surveillance et, en dernier ressort, par des actionnaires particuliers).

Chacun de ces sous-types généraux (et une myriade de variations) implique des formes de gestion particulières. Les populations autochtones et les communautés locales peuvent aussi être les propriétaires officiels et/ou contrôler la terre et les ressources qu'elles veulent conserver. Leur cas vient juste d'être discuté ci-dessus.

\section{Aires protégées privées dans les catégories}

Les aires protégées privées peuvent appartenir à chacune des catégories, et c'est le cas. Certaines personnes estiment qu'elles seront mieux représentées dans les catégories IV à VI, mais en fait, beaucoup correspondent aux objectifs de gestion des catégories I à III, peut-être particulièrement celles qui appartiennent ou sont gérées par des ONG. Bien que la plupart des aires marines protégées ne soient pas privées, un nombre croissant d'îles privées sont protégées, avec leurs zones côtières et marines.

La plupart des aires protégées ne sont pour le moment pas inscrites dans la BDMAP et restent donc largement méconnues de la communauté mondiale ; elles sont aussi souvent réellement ignorées par les gouvernements et ne sont pas incluses 
dans la planification nationale ou écorégionale. Ceci peut refléter un manque de capacités de la part du gouvernement pour récolter les données sur les aires protégées privées ou le fait que les gestionnaires/propriétaires de l'aire protégée sont réticents à l'idée de partager librement les informations.

\section{« Moyens efficaces "}

Dans la majorité des cas, la création d'une aire protégée privée - et sa gestion dans un objectif de conservation - est une démarche volontaire de la part des propriétaires. Une reconnaissance croissante des opportunités qu'il y a d'atteindre des objectifs de conservation dans un domaine privé - et spécialement la prolifération de mécanismes et d'incitants pour ce faire - a entraîné une augmentation spectaculaire du nombre et de la superficie des aires protégées privées. Ces mécanismes et incitants incluent :

- Des systèmes de désignations volontaires d'aires protégées, par lesquels les propriétaires acceptent certains objectifs de gestion ou des restrictions en échange d'appuis ou d'autres incitants : les Réserves privées du patrimoine mondial au Brésil en sont un exemple.

- L'abandon volontaire de droits d'affectation du sol sur une propriété privée, parfois pour obtenir des avantages (par exemple dans un terrain voisin) octroyés pour compenser la perte de valeur théorique, pour en assurer la protection définitive, ou comme mesures de compensation : les mécanismes incluent des droits de passage pour la conservation avec les conventions et servitudes qui y sont liées, ainsi que des accords de gestion de la conservation.

- Contributions désintéressées, par lesquelles des ONG récoltent des fonds privés ou publics pour l'acquisition de terres à protéger ou reçoivent des dons sous forme de terres directement de donateurs volontaires : ceci inclut de grandes ONG internationales comme The Nature Conservancy et Conservation International ainsi que de nombreux exemples nationaux et locaux.

- Des propriétés de sociétés mises en jachère, des donations, ou la gestion d'une aire à des fins de conservation, encouragées par un souhait de bonnes relations publiques ; comme concessions ou compensations pour d'autres activités ; parce que c'est stipulé dans la certification "verte »; comme un investissement pour l'avenir ; ou à cause de l'intérêt individuel du personnel.

- L'abandon involontaire de certains droits de gestion en réponse à des restrictions juridiques.

Le système des catégories implique la possibilité d'aider les gouvernements à contrôler les activités de conservation privée, en évaluant aussi bien les objectifs de la gestion des aires protégées privées que son efficacité. Il existe aussi des garanties locales et nationales dans certains pays pour s'assurer que les aires protégées privées sont bien gérées selon leur désignation, réglementation et déclaration. La signification pratique et la mise en place de ces garde-fous varie considérablement selon les pays. (Il existe aussi des exemples d'autorégulation d'aires protégées privées, comme le Programme d'accréditation des propriétés foncières qui se développe aux Etats-Unis). Lapplication du système des catégories de l'UICN, décrit dans ces lignes directrices, pourrait offrir aux gouvernements une base de référence pour faire le suivi des aires protégées privées dans le cadre de leurs stratégies de conservation nationales.

La définition d'une aire protégée par l'UICN dit clairement que ces aires doivent être gérées pour la conservation à perpétuité, et c'est le principal critère qui va distinguer si une aire particulière de terre ou d'eau privée est, ou non, une aire protégée. Un propriétaire qui gère pour la conservation aujourd'hui mais ne prend pas de dispositions pour savoir si la gestion va se poursuivre à l'avenir contribue, certes, à la conservation mais pas au moyen de ce que l'on reconnaît comme une aire protégée. S'assurer de la sécurité à long terme est un des défis à relever par les aires protégées privées. Certains gouvernements nationaux se sont préoccupés de cette question en introduisant une législation qui fait de la déclaration d'une aire protégée privée un engagement légalement contraignant dans le temps, même si, là où ce n'est pas le cas, d'autres mécanismes peuvent s'avérer nécessaires. Ceux-ci sont en développement et comprennent divers systèmes de certification, des systèmes institutionnalisés de déclaration et de pression par les pairs. Il est urgent de travailler encore sur les étapes nécessaires pour intégrer complètement les aires protégées privées dans les systèmes nationaux et internationaux d'aires protégées. 



\section{Appliquer les catégories}

Cette section décrit les processus d'application des catégories, y compris : choisir et convenir de la catégorie la plus adéquate pour une situation donnée ; attribuer la catégorie de manière à satisfaire aux exigences légales et aux normes et standards internationaux; et enregistrer l'aire et sa catégorie auprès du Centre mondial du suivi de la conservation du PNUE. Les questions au sujet de la vérification des catégories et du traitement des disputes sont aussi discutées. 


\section{Choisir la bonne catégorie}

Une fois qu'une aire a été identifiée comme aire protégée selon la définition de l'UICN, l'étape suivante de sa classification consiste à déterminer quelle catégorie correspond le mieux aux objectifs généraux de sa gestion.

Puisque le système de catégories reflète les objectifs de gestion, il s'ensuit que dès qu'une décision a été prise au sujet de la gestion d'une aire protégée, la catégorie adéquate devrait être évidente. C'est quelquefois comme cela que ça se passe. Malheureusement, dans de nombreux autres cas, une bonne part de confusion persiste : cela peut être parce qu'il y a de multiples objectifs pour une même aire protégée (parfois dans différentes parties de l'aire), ou parce que les objectifs de l'aire protégée évoluent et deviennent plus complexes, ou encore parce qu'il subsiste des incertitudes quant à l'approche particulière qui sera la meilleure. Convenir des objectifs (éventuellement en réévaluant les objectifs originaux) et développer des plans de gestion sont deux activités étroitement liées à l'adoption d'une catégorie.

De nombreuses personnes ont demandé à l'UICN un moyen infaillible pour identifier une catégorie, mais c'est difficile. Il existe souvent plusieurs façons d'approcher la gestion dans une même aire protégée qui peut donc être catégorisée de différentes manières. Que se passe-t-il lorsque la plus grande partie d'une aire protégée est gérée de telle façon et le reste de telle autre ? Existe-t-il une taille minimale et maximale pour des catégories particulières? Les désignations internationales telles que le Patrimoine mondial ou Ramsar sont-elles liées à des catégories particulières ? Quel niveau d'activités humaines est " autorisé " dans les aires protégées des différentes catégories ? La section suivante tente de répondre à ces questions.

Il faut se rappeler que de nombreux pays ont des législations qui définissent clairement les critères permettant de distinguer différents types d'aires protégées : ils peuvent, ou non, être équivalents aux catégories de l'UICN. Quand ce n'est pas le cas, les pays qui veulent inscrire leurs aires protégées correctement dans la BDMAP doivent clarifier la relation entre leur propre classification et le système de catégories de l'UICN - ce que beaucoup ont déjà fait. Dans d'autres cas, les pays ont pris les catégories de l'UICN et les ont affinées pour les adapter aux conditions spécifiques du pays. Pour autant que ce processus ne dénature pas les principes de base d'une aire protégée ou des catégories spécifiques, l'UICN encourage ce genre de procédure. Il en résulte que le choix d'une catégorie peut varier avec les conditions et d'un pays à l'autre et peut, à l'occasion, être un processus très compliqué, parfois autant un art qu'une science.

Mais avant de sauter dans les détails techniques de l'application des catégories d'aires protégées, il est utile de se demander pour$q u o i$ on choisit des catégories. La catégorisation peut se faire à trois étapes de la vie d'une aire protégée, et même si cela ne devrait pas influencer le résultat, cela peut faire une énorme différence dans le processus. Les catégories peuvent être choisies :

- Avant que l'aire protégée soit établie, quand les décisions en matière d'objectifs de gestion doivent faire partie du processus de planification.

- Après que l'aire protégée a été établie, lorsque les objectifs de gestion sont déjà décidés et que le choix de la catégorie consiste à trouver celle qui correspond le mieux à l'aire protégée dans son ensemble ; même si l'examen des catégories à ce stade peut aussi encourager certains changements dans les objectifs et les activités de la gestion.

- Dans une aire protégée qui est déjà classée dans une catégorie mais où soit la gestion change pour traiter certains problèmes ou priorités émergents, soit il y a des doutes concernant le choix de la bonne catégorie. Cependant, le changement de catégorie dans la plupart des pays est régi par le cadre juridique sur les aires protégées et doit passer par une évaluation au moins aussi rigoureuse que celle qui s'est faite lorsqu'il s'est agi de définir la catégorie au départ.

\section{Comment l'objectif de gestion est-il lié à la catégorie?}

- La catégorie doit être fondée sur l'(es) objectif(s) premier(s) de la gestion de l'aire protégée

- L'objectif premier de la gestion doit s'appliquer au moins aux trois-quarts de l'aire protégée

LA CATEGORIE DOIT SE FONDER SUR L'(ES) OBJECTIF(S) PREMIER(S) DE LA GESTION : dont la liste est donnée pour chaque catégorie au chapitre 2. (Elle doit aussi respecter la définition d'une aire protégée). Ceci suppose que l'agence responsable de l'aire protégée est à même de décider du but principal de la gestion. Ceci n'est pas forcément un choix facile à faire, mais d'autre part, ne pas pouvoir le faire signifierait que la gestion elle-même peut être indécise et donc probablement inefficace. En principe, un bon processus d'évaluation pour identifier la bonne catégorie devrait impliquer les parties prenantes clés et d'autres organismes s'occupant de la conservation et de la gestion de l'aire protégée et devrait se baser sur les connaissances scientifiques et sociales les plus pointues. Identifier un objectif premier ne signifie pas que les autres buts ne sont pas importants : pratiquement toutes les aires protégées renferment de multiples valeurs. En pratique, il n'est pas toujours facile d'émettre un jugement - les exemples suivants passent en revue certaines des questions qui se présentent fréquemment :

- Ecosystème ou habitat - catégorie II ou IV ? Les aires protégées de catégorie II sont supposées préserver des écosystèmes entiers alors que la catégorie IV vise à préserver des espèces ou des fragments d'écosystème. En 
fait, peu d'aires protégées sont assez vastes pour protéger des écosystèmes entiers, avec les voies de migrations, les fonctions hydrologiques, etc. qui leur sont associées. La distinction entre la catégorie II et la IV est donc une affaire de degré : une aire protégée de catégorie II doit viser à protéger la majorité des fonctions écosystémiques naturelles, alors qu'une aire de catégorie IV est d'habitude soit un fragment d'écosystème (p.ex. un bassin, un fragment de récif corallien, une petite surface de tourbière), soit une aire qui dépend d'une intervention régulière de gestion pour préserver un écosystème artificiel (p.ex. une forêt de taillis ou une surface de prairie régulièrement fauchée). Les aires protégées de catégorie IV sont généralement plus petites que celles de la catégorie II même si ce n'est pas une règle absolue et qu'il existe aussi des aires protégées de catégorie IV de grande taille.

- Intervention de la gestion ou paysage culturel catégorie $\boldsymbol{I V}$ ou $\boldsymbol{V}$ ? Une aire protégée de catégorie IV est d'abord gérée pour ses valeurs floristiques et faunistiques et des interventions comme la taille, des éclaircies dans la végétation, des feux programmés, etc. se font principalement dans cet objectif ; tout profit ou bénéfice social retiré de ces activités n'est que secondaire. Les interventions de la gestion dans les aires protégées de la catégorie $\mathrm{V}$ sont, par contre, destinées à soutenir les moyens de subsistance et ne sont pas seulement une partie de la stratégie de gestion de la biodiversité. Une aire protégée de catégorie $\mathrm{V}$ utilise donc des systèmes de gestion culturels qui ont aussi une valeur du point de vue de la biodiversité, comme une forêt de chênes-lièges qui serait gérée d'abord pour le liège mais qui serait aussi importante pour la vie sauvage si elle est intégrée dans une approche de la conservation par paysage. Dans la plupart des aires protégées de catégorie $\mathrm{V}$, différentes approches de gestion sont souvent combinées.

- Restauration d'un paysage culturel - catégorie V ou quelque chose d'autre ? Un paysage culturel appartiendrait normalement à la catégorie V. Mais si le but de la gestion est de restaurer un ancien paysage culturel en quelque chose de plus naturel, alors l'objectif de la gestion et, dès lors, la catégorie peuvent correspondre plutôt à quelque chose d'autre, comme la catégorie Ib ou encore II ou IV. Par exemple, une forêt résiduelle utilisée auparavant pour le pâturage de moutons et que l'on protège pour la restaurer en quelque chose qui ressemble à l'écosystème forestier d'origine ne sera pas d'habitude classée comme une aire protégée de catégorie V. Protéger un récif corallien très exploité dans le but de le restaurer en un écosystème plus naturel ne lui permettra pas non plus, normalement, d'être classé dans la catégorie V.

- Monument naturel ou écosystème - catégorie III ou II ? Quand la protection d'un monument naturel équivautelle à celle d'un écosystème ? En pratique, c'est souvent une question de taille et de priorité dans les objectifs de gestion. Une aire protégée qui contient un monument naturel important (normalement catégorie III) mais qui, néanmoins, est gérée d'abord pour ses fonctions écosystémiques (normalement catégorie II), devrait être classée dans la catégorie II plutôt que III - p.ex. le Grand Canyon de l'Arizona est un des plus vastes monuments naturels au monde mais le parc national est géré d'abord pour ses fonctions écosystémiques et classé dans la catégorie II.

- Utilisation durable ou utilisation accessoire par les communautés locales - quand utiliser la catégorie VI ? De nombreuses catégories d'aires protégées autorisent des utilisations humaines limitées. Par exemple, de nombreuses zones de nature sauvage (Ib) et d'écosystèmes protégés (II) permettent aux populations locales de mener leurs activités de subsistance traditionnelles à petite échelle qui sont en harmonie avec la nature dans les aires protégées, telles que (en fonction des accords de gestion individuels) le maintien de troupeaux de rennes, la pêche, la collecte de produits forestiers non ligneux et une chasse de subsistance modérée. Mais dans ces cas-là, l'objectif est la conservation de la vie sauvage ou des écosystèmes, et les prélèvements humains devraient avoir sur eux un impact minimal. Dans la catégorie VI, l'objectif de la gestion est l'utilisation durable en synergie avec la conservation de la nature, et l'on s'attend à ce que les activités soient gérées d'une façon qui ne produit pas d'impact substantiel sur ces écosystèmes. La différence est donc partiellement une question de degré.

- Paysage culturel - qu'est-ce qui ne rentre pas dans la catégorie $V$ ? Peu d'aires protégées, s'il en existe, n'ont pas été modifiées par des sociétés humaines pendant des centaines ou des milliers (voire des dizaines de milliers) d'années, et de nombreux écosystèmes aquatiques ont aussi été modifiés. On pourrait arguer que toutes les aires protégées du monde relèvent de la catégorie V. Mais tout en reconnaissant le rôle des communautés humaines, l'UICN fait la distinction entre les aires qui renferment majoritairement des espèces et des écosystèmes naturels (d'habitude pas la catégorie V) de celles où le niveau de modification est plus élevé, comme les aires avec des exploitations agricoles installées depuis longtemps ou d'autres processus de gestion qui ont causé des changements majeurs à l'écologie et à la diversité des espèces (d'habitude catégorie V).

LOBJECTIF PREMIER DE LA GESTION DOIT POUVOIR S'APPLIQUER AU MOINS AUX TROISQUARTS DE L'AIRE PROTÉGÉE - LA RĖGLE DES 75 POUR CENT : de nombreuses aires protégées peuvent contenir des zones spécifiques où d'autres utilisations sont autorisées : p.ex. :

- Les lodges et les camps touristiques dans les parcs nationaux de catégorie II - comme c'est le cas dans de nombreuses aires protégées de la savane africaine ; 
- Les villages qui subsistent dans des aires qui sont, par ailleurs, strictement protégées - p.ex. il reste un village dans le Parc National de Cat Tien, au Viet Nam ;

- Les petites aires centrales strictement protégées dans ce qui est, à part cela, un paysage culturel géré selon la catégorie $\mathrm{V}-$ p.ex. les forêts appartenant au National Trust dans le Parc National des Brecon Beacons, au pays de Galles, en Angleterre;

- Les aires où la pêche est autorisée dans ce qui est, par ailleurs, une aire marine protégée ou une aire protégée d'eau douce - p.ex. dans la Kosi Bay Nature Reserve au KwaZulu Natal, en Afrique du Sud.

L'UICN reconnaît cet état de fait et recommande que 25 pour cent maximum de la surface terrestre ou aquatique d'une aire protégée soient gérés suivant d'autres objectifs pour autant que ceux-ci soient compatibles avec l'objectif premier de l'aire protégée. Dans certains cas, les 25 pour cent sont mobiles : par exemple, la Bwindi Impenetrable Forest National Park, en Ouganda, permet aux communautés locales de récolter des plantes médicinales et d'autres produits forestiers non ligneux dans des zones spécialement désignées qui sont déplacées de temps en temps pour garantir que la collecte de ces plantes ne soit pas excessive.

\section{Comment la catégorie est-elle affectée par la taille de l'aire protégée ?}

- II n'existe pas de règles fermes et définitives, mais les aires de certaines catégories ont tendance à être relativement plus vastes ou plus petites

La taille générale dépend souvent d'autres facteurs, comme la surface de terres ou d'eau disponible, la densité de la population, etc. En termes de taille relative, les aires de certaines catégories sont plus susceptibles d'être plus vastes, ou plus petites, en raison de leurs objectifs de gestion particuliers, mais il peut y avoir des exceptions dans pratiquement toutes les catégories. Pour aider à la sélection, le Tableau 4, ci-dessous, suggère une échelle relative pour les catégories et en explique la raison, mais il mentionne aussi certaines exceptions pour montrer que la taille seule n'est pas un facteur déterminant.

Tableau 4. Comment la taille d'une aire protégée est liée à la catégorie

\begin{tabular}{|c|c|c|c|}
\hline Catégorie & Taille relative & Explication & Exceptions \\
\hline la & $\begin{array}{l}\text { Souvent } \\
\text { petite }\end{array}$ & $\begin{array}{l}\text { Strictement protégée, il est toujours difficile } \\
\text { de s'accorder au sujet des zones interdites } \\
\text { d'accès sauf dans les zones peu habitées : } \\
\text { donc, même s'il existe de vastes aires la } \\
\text { (p.ex. en Australie), ce sont probablement } \\
\text { des exceptions. }\end{array}$ & $\begin{array}{l}\text { Des vastes aires dans des } \\
\text { endroits avec une faible densité de } \\
\text { population humaine et peu d'intérêt } \\
\text { pour le tourisme. }\end{array}$ \\
\hline lb & $\begin{array}{l}\text { D'habitude } \\
\text { grande }\end{array}$ & $\begin{array}{l}\text { Une partie de la raison d'être des zones de } \\
\text { nature sauvage réside dans le fait qu'elles } \\
\text { offrent assez d'espace pour y trouver la } \\
\text { solitude et un écosystème de grande taille. }\end{array}$ & $\begin{array}{l}\text { Des aires relativement petites créées } \\
\text { pour la nature sauvage dans l'espoir } \\
\text { qu'elles pourront être étendues dans } \\
\text { le futur. }\end{array}$ \\
\hline II & $\begin{array}{l}\text { D'habitude } \\
\text { grande }\end{array}$ & $\begin{array}{l}\text { La préservation des processus } \\
\text { écosystémiques suppose que l'aire doit être } \\
\text { suffisamment vaste pour contenir tous ces } \\
\text { processus ou la plupart d'entre eux. }\end{array}$ & $\begin{array}{l}\text { De petites îles peuvent être } \\
\text { réellement des écosystèmes et } \\
\text { donc relever fonctionnellement de la } \\
\text { catégorie II. }\end{array}$ \\
\hline III & $\begin{array}{l}\text { D'habitude } \\
\text { petite }\end{array}$ & $\begin{array}{l}\text { Les grands sites qui contiennent des } \\
\text { monuments naturels protègeront } \\
\text { généralement aussi d'autres valeurs (p.ex. } \\
\text { des valeurs de l'écosystème et/ou de la vie } \\
\text { sauvage). }\end{array}$ & \\
\hline IV & $\begin{array}{l}\text { Souvent } \\
\text { petite }\end{array}$ & $\begin{array}{l}\text { Si le site est créé pour protéger uniquement } \\
\text { des espèces ou des habitats particuliers, } \\
\text { cela implique qu'il est relativement petit. }\end{array}$ & $\begin{array}{l}\text { Les vastes aires désignées comme } \\
\text { réserves naturelles mais qui } \\
\text { requièrent une gestion régulière pour } \\
\text { continuer à fonctionner seront plus } \\
\text { indiquées dans la catégorie IV. }\end{array}$ \\
\hline V & $\begin{array}{l}\text { D'habitude } \\
\text { grande }\end{array}$ & $\begin{array}{l}\text { La mosaïque de différentes approches vient } \\
\text { s'ajouter aux bénéfices pour la conservation } \\
\text { des approches par paysage, ce qui suggère } \\
\text { une aire plus vaste. }\end{array}$ & $\begin{array}{l}\text { Certaines mini-réserves créées pour } \\
\text { des espèces sauvages à l'origine } \\
\text { des plantes cultivées ou de races } \\
\text { rustiques peuvent nécessiter une } \\
\text { gestion culturelle. }\end{array}$ \\
\hline VI & $\begin{array}{l}\text { D'habitude } \\
\text { grande }\end{array}$ & $\begin{array}{l}\text { La nature extensive de la gestion suggère } \\
\text { qu'il s'agira souvent d'aires de grande taille. }\end{array}$ & $\begin{array}{l}\text { Certaines aires marines protégées } \\
\text { de la catégorie VI sont petites. }\end{array}$ \\
\hline
\end{tabular}




\section{Une aire protégée peut-elle contenir plus d'une catégorie?}

- Certaines aires protégées distinctes nichées au cœur d'aires protégées plus grandes peuvent avoir leur propre catégorie ;

- Différentes zones dans de plus grandes aires protégées peuvent avoir leur propre catégorie si les zones sont décrites et fixées par la loi ;

- Différentes aires protégées constituant une aire protégée transfrontalière peuvent avoir différentes catégories.

C'est une des questions les plus épineuses concernant les catégories. La réponse est que cela dépend : de la propriété, de la gouvernance et, dans une certaine mesure, des souhaits de l'autorité ou des autorités de l'aire protégée.

Il y a trois situations où une aire protégée ou des aires protégées contiguës peuvent se voir attribuer des catégories différentes :

Aires incluses avec de multiples objectifs : des aires protégées de différentes catégories sont parfois incluses l'une dans l'autre - c.-à-d. qu'une aire protégée de grande taille peut contenir plusieurs aires protégées plus petites. Le modèle le plus commun serait une aire vaste, moins strictement protégée qui en contient d'autres, plus petites et protégées plus strictement. Par exemple, de nombreuses aires de catégorie $\mathrm{V}$ contiennent des aires de catégorie I et IV - dépendant éventuellement d'autorités de gestion ou d'approches de gouvernance complètement différentes. Le Parc Naturel Régional du Vercors (catégorie V), en France, contient les Hauts Plateaux du Vercors (catégorie IV). Ceci est en parfaite adéquation avec l'application du système des catégories. Lorsque l'on fait le compte-rendu des aires protégées incluses, il est important d'éviter un double comptage et de s'assurer que les bases de données ne surestiment pas la superficie de terre ou d'eau qui a été désignée. Par exemple, au Royaume-Uni, les parcs nationaux (catégorie $\mathrm{V}$ ), qui couvrent près de neuf pour cent de la surface terrestre de l'Angleterre et du pays de Galles, contiennent un certain nombre de réserves naturelles nationales (catégorie IV), qui couvrent environ 0,7 pour cent de la surface des parcs nationaux.

Différentes zones au sein des aires protégées : le zonage est d'habitude un outil de gestion au sein d'une même aire protégée et il n'est généralement pas identifié par une catégorie différente, mais il y a des exceptions. Dans certaines aires protégées, des portions d'une même unité de gestion sont classées par la loi comme ayant des objectifs de gestion différents et comme étant des aires protégées distinctes : en effet, ces "portions " sont des aires protégées individuelles qui, ensemble, composent une unité plus grande, même si elles relèvent toutes de la même autorité de gestion. Dans le cas de l'Australie, par exemple, le zonage est utilisé comme un instrument de gestion et comme un outil servant à la désignation d'une aire protégée et il est inscrit dans la réglementation. Donc, le Great Barrier Reef Marine Park d'Australie s'est vu assigner la catégorie VI dans son ensemble, mais il s'est aussi vu assigner officiellement d'autres catégories qui concernent la gestion réglementée de certaines zones dans le parc. Séparer des zones sous différentes catégories, c'est quelque chose qui ne sera généralement tenté que pour de vastes aires protégées et qui est laissé à la discrétion du gouvernement concerné étant donné les conditions décrites ci-dessus.

L'UICN recommande que l'on puisse rapporter de multiples catégories au sein d'une seule grande aire protégée lorsque certaines conditions sont remplies. Ces conditions reflètent la permanence et les objectifs du système de zonage. Les scénarios alternatifs sont les suivants :

- Zones "dures": des zones peuvent être assignées à une catégorie de l'UICN lorsqu'elles (a) sont clairement cartographiées, (b) sont reconnues par des moyens juridiques ou autres et (c) ont des objectifs de gestion distincts et non ambigus qui peuvent être assignés à une catégorie particulière d'aire protégée (la règle des 75 pour cent n'est pas pertinente ici);

- Zones "douces": des zones ne sont pas assignées à une catégorie de l'UICN quand elles (a) font l'objet de révisions régulières, comme par un processus de planification de la gestion, (b) ne sont pas reconnues par des moyens efficaces, ni juridiques, ni autres et (c) ne correspondent pas à une catégorie particulière d'aire protégée (la règle des 75 pour cent s'applique pour définir la catégorie générale de l'aire protégée).

Pour être clair, une catégorisation séparée des zones est possible lorsque la législation de base décrit et délimite des zones au sein d'une aire protégée et pas lorsque la législation de base permet simplement de faire un zonage dans l'aire protégée, par exemple lors d'un processus de planification de gestion. L'UICN déclare dans la plupart des cas qu'il n'est pas nécessaire d'attribuer une catégorie à des zones dans des aires protégées mais que cela peut être pertinent dans les plus grandes aires protégées où des zones précises sont elles-mêmes des aires protégées importantes de plein droit.

Aires protégées transfrontalières : dans un nombre croissant de cas, des aires protégées couvrent les deux côtés d'une frontière nationale ou fédérale ; elles sont gérées par des autorités différentes, mais avec un certain niveau de coopération, qui varie d'arrangements informels à des accords formels entre gouvernements ; ce sont les aires protégées transfrontalières (Sandwith et al. 2001). Souvent, les aires protégées contiguës peuvent être gérées différemment et, par conséquent, elles relèveront de différentes catégories. S’il est certes important que les approches de gestion dans les différentes composantes d'une aire protégée transfrontalière 
soient complémentaires, il n'y a aucune raison pour qu'elles soient les mêmes.

La Figure 2 donne un exemple d'arbre décisionnel pour décider si une zone convient pour avoir sa propre catégorie.

\section{Comment la propriété et la responsabilité de la gestion ont-elles un impact sur les catégories?}

- La catégorie n'est pas affectée par le type de propriété ou de gouvernance
Toute structure de propriété, tout type de gouvernance peut se trouver dans chaque catégorie, et l'on peut trouver des exemples de toutes les combinaisons possibles partout dans le monde. Il existe des tendances : de vastes aires vouées à la protection de l'écosystème, comme dans la catégorie II sont plus probablement des propriétés de l'Etat gérées par lui, alors que les aires préservées par les communautés sont plus susceptibles d'appartenir aux catégories moins restrictives que sont la $\mathrm{V}$ et la VI, mais il y a des exceptions. Par exemple, certaines des aires les plus strictement protégées au monde sont des sites naturels sacrés où l'entrée est interdite à tous à l'exception de quelques personnes spécialement nommées, et dans certains cas même, aucun humain ne peut entrer.

Figure 2. Zones et catégories d'aires protégées de l'UICN

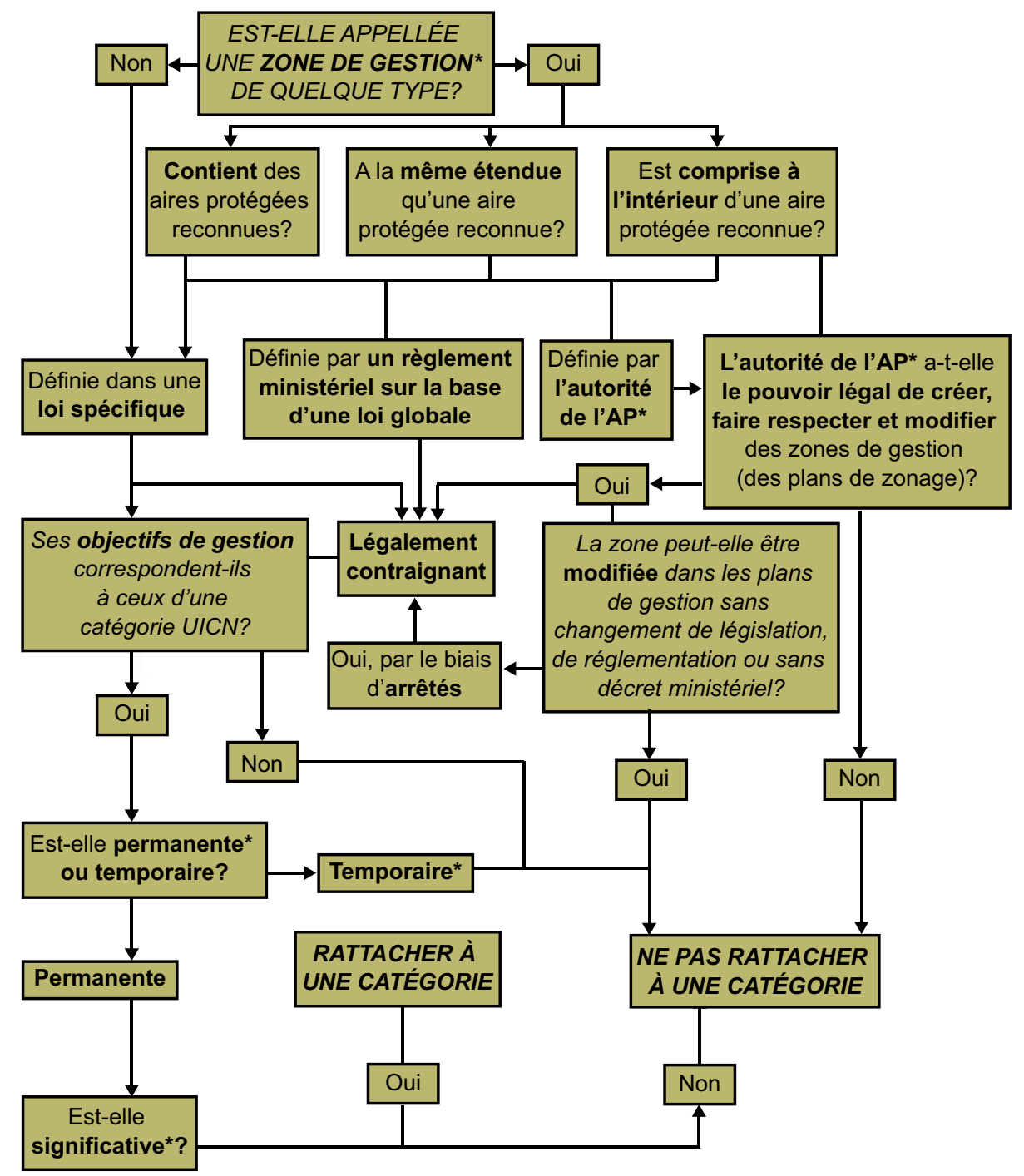

* Zone de gestion - p.ex. zone tampon, zone de nature sauvage, zone de loisirs, zone sans prélèvement, zone noyau, etc. Autorité de l'aire protégée - Département ministériel, agence, ONG ou institution communautaire reconnue par la loi Permanente - inscrite dans la loi, établie et reconnue, objet d'une vision à long terme ( $p$.ex. zone noyau pour des espèces reproductrices clés)

Temporaire - établie à des fins de gestion uniquement, (pour une durée limitée)

Significative - d'une ampleur et/ou d'une proportion admissible ou raisonnable par rapport au paysage 


\section{Qu'en est-il des zones qui entourent des aires protégées?}

- Les zones tampons, les corridors biologiques, etc. peuvent, ou pas, être aussi des aires protégées (et à ce titre se voir attribuer une catégorie) en fonction du mode de gestion et de sa reconnaissance par l'Etat.

Les planificateurs de la conservation soulignent l'importance de connecter entre elles les aires protégées par des corridors biologiques ou des étapes-relais (des habitats accueillants utilisés par des espèces migratrices) et aussi de les isoler avec des zones tampons. Malheureusement, la compétition pour des terres, la pression démographique et une gouvernance médiocre signifient que de nombreuses aires protégées restent des « îlots » isolés. Pallier ces faiblesses par des projets de restauration, des mesures compensatoires, des retraits de terre, des accords volontaires et des changements législatifs est un défi à long terme. Pour savoir si ces aires peuvent se voir assigner une catégorie, il faut d'abord savoir si elles sont qualifiées au titre d'aire protégée selon la définition de l'UICN. Certaines aires protégées de catégorie $\mathrm{V}$ ont été créées pour servir de zones tampons autour d'aires plus strictement protégées. D'autres zones tampons et des corridors biologiques ne sont pas des aires protégées mais plutôt des aires où une combinaison d'accords volontaires et/ou de mesures compensatoires aide à protéger l'intégrité de l'aire protégée par des approches par paysage et la préservation de la connectivité. Par exemple, dans certains pays, certaines plantations d'arbres ou des forêts naturelles gérées aident à isoler des aires protégées en empêchant la conversion des terres ; mais aucune de ces utilisations ne justifierait la qualification d'aire protégée.

\section{Comment les autres désignations internationales de protection sont-elles liées aux aires protégées et aux catégories de I'UICN?}

- La plupart des autres désignations internationales de protection ne sont pas nécessairement des aires protégées reconnues par l'UICN, même si, en pratique, beaucoup sont des aires protégées ;

- Les sites du patrimoine mondial, les sites Ramsar et les sites Natura 2000 peuvent être de n'importe quelle catégorie de l'UICN, ou d'aucune ;

- Les réserves de biosphère doivent avoir un noyau très protégé (catégorie I à IV) et être entourées d'une zone de gestion durable (catégorie V/VI ou une aire qui n'est pas complètement protégée).

Il existe toute une gamme d'efforts à l'échelle mondiale ou régionale pour définir la conservation relative à des surfaces de terre et d'eau, y compris :
- Le Patrimoine mondial de l'UNESCO - sites naturels et sites mixtes naturels et culturels reconnus par le Comité du PM comme étant de "valeur universelle exceptionnelle";

- Le Programme sur L'homme et la biosphère (MAB) de l'UNESCO - les réserves de biosphère sont des sites où l'utilisation durable est intégrée à la conservation ;

- Les sites Ramsar - d'importantes zones d'eau douce ou de marées inscrites sur la liste de la Convention de Ramsar.

Etablir la relation entre ces sites et les aires protégées de l'UICN est compliqué et ce sera décrit en détail dans une des sections suivantes. Pour certains des ceux qui précèdent (p.ex. les sites naturels du patrimoine mondial), la plupart des sites listés sont aussi des aires protégées. Certains pays considèrent automatiquement de telles désignations comme des aires protégées, d'autres pas. La tendance générale semble être de considérer que le fait d'assigner un statut complet d'aire protégée à ces désignations est souvent le meilleur moyen d'assurer, à long terme, la conservation des valeurs du site. Ceci étant, d'autres désignations peuvent contenir des sites de toutes les catégories de l'UICN : il n'y a pas de lien particulier entre une reconnaissance telle que le statut de patrimoine mondial et une quelconque catégorie ou groupe de catégories de l'UICN.

Une exception possible concerne les réserves de biosphère MAB qui encouragent l'utilisation durable autour d'un noyau terrestre ou aquatique très protégé. En général, une réserve de biosphère comprend : (a) une zone noyau très protégée (d'habitude catégorie I à IV) ; (b) une zone tampon qui peut être de catégorie V ou VI, ou au contraire une portion de terre ou d'eau gérée qui ne correspond à aucune catégorie de l'UICN ; et (c) une zone de transition qui ne correspond à aucune catégorie de l'UICN.

\section{Assignation}

Le processus d'assignation est devenu plus significatif à mesure que les catégories commençaient à être appliquées comme instruments politiques aussi bien que comme moyens de mesures. Par exemple, lorsque l'assignation d'une catégorie donnée porte en elle-même des restrictions légales d'utilisation de la terre ou de l'eau, ou qu'elle dicte qui peut et qui ne peut pas vivre dans l'aire, comme c'est le cas dans certains pays, alors la décision concernant une catégorie donnée est plus significative que lorsqu'elle sert simplement d'outil statistique. Le processus d'assignation revient au pays ou à l'organisation gouvernementale concernée, mais la section suivante expose certains principes et propose une méthodologie.

\section{Certains principes pour l'assignation}

Lapproche par l'UICN de l'assignation des catégories de gestion des aires protégées se fonde sur une série de principes, 
présentés ci-dessous, liés à la responsabilité, l'implication des parties prenantes et les garanties :

- Responsabilité : l'usage des catégories est volontaire, et aucun organisme n'a le droit de les imposer. Les états détiennent en général la décision finale, ou au moins une responsabilité globale, quant aux utilisations des terres et de l'eau, et il est donc raisonnable que ce soient eux qui décident aussi de la catégorie d'une aire protégée.

- Démocratie : néanmoins, l'UICN demande aux états de consulter les parties prenantes concernées dans le processus d'assignation d'une catégorie. Des propositions sont faites ci-dessous. Les processus de démocratisation et de décentralisation font qu'un nombre croissant de gouvernements sous-nationaux prennent en charge la responsabilité des aires protégées; le gouvernement local ou régional rapporte d'habitude au gouvernement central. Dans la plupart des aires privées ou conservées par les communautés, les gouvernements vont souvent s'en remettre pour l'assignation à l'opinion de l'organisation qui les possède et les administre, même si certains pays disposent de politiques ou de lois pour ce faire.

- Procédure de doléances : de nombreuses parties prenantes soutiennent l'idée qu'il devrait exister un moyen de contester les décisions concernant les catégories. L'UICN est d'accord avec cette idée, et note que les décisions finales concernant la gestion reviennent toujours à l'Etat ou au propriétaire. Certaines propositions en cas de plaintes éventuelles sont expliquées plus bas.

- Gestion des données : les informations concernant les aires protégées, y compris leur catégorie, devraient être rapportées au Centre mondial de surveillance continue de la conservation de la nature du PNUE qui coordonne la Base de données mondiale sur les aires protégées et compile la Liste des $N U$ des aires protégées.

- Vérification : l'UICN peut donner des conseils pour les assignations et elle mène parfois des missions consultatives individuelles dans des pays ou des aires protégées particulières. L'UICN envisage aussi le développement d'une certaine forme de vérification ou de certification pour les catégories d'aires protégées, sur une base volontaire, lorsque l'autorité de gestion souhaite faire vérifier que les objectifs de gestion correspondent bien à la catégorie assignée.

\section{Un processus pour l'assignation}

Il est conseillé de fonder l'assignation sur quatre éléments principaux :

- Une bonne orientation pour les gouvernements et autres autorités des aires protégées ;

- Un processus d'assignation reconnu ;
- Un système pour contester les catégories assignées, à développer ;

- Un processus de vérification ; qui pourrait être faire au niveau national (par un panel d'experts, par exemple) ou demandé auprès d'un organisme indépendant comme l'UICN.

Les trois premiers sont discutés ci-dessous : actuellement, il n'existe aucun système de vérification mais il peut être mis au point dans le futur.

\section{Bonne orientation pour les gouvernements et les autres autorités des aires protégées}

La base de l'utilisation des catégories, ce sont les orientations contenues dans cette publication. De plus, d'autres orientations plus détaillées concernant des questions spécialisées sont disponibles ou le seront, par exemple en ce qui concerne :

- Les biomes : aires protégées forestières (Dudley et Phillips 2006), marines, d'eaux douces intérieures, etc. ;

- Les catégories : semblables aux notes d'orientation développées pour la catégorie V (Phillips 2002), déjà prévues pour les catégories Ib et VI ;

- Les régions : semblables aux notes d'orientation déjà produites en Europe (EUROPARC et UICN 1999) et prévues pour plusieurs autres régions, soit sous forme de lignes directrices, soit comme études de cas ;

- Les outils de sélection : pour identifier la catégorie et le type de gouvernance ;

- Les types de gouvernance : il existe aussi un intérêt certain pour la production d'informations plus détaillées sur les aires protégées privées, les zones de conservation communautaire et les aires protégées des populations autochtones.

\section{Un processus agréé pour l'assignation}

La Figure 3 ci-dessous montre une proposition de processus pour l'assignation : idéalement, il peut impliquer de nombreuses parties prenantes, particulièrement lorsque l'assignation à une catégorie particulière aura des impacts sur les personnes qui vivent dans ou à proximité de l'aire protégée, ou sur d'autres parties prenantes. Une des options serait d'avoir un groupe de travail national qui revoit les données sur les aires protégées, et l'on a suggéré qu'un comité national pour l'UICN pourrait en être le véhicule évident. La mesure dans laquelle les parties prenantes sont impliquées dans ces décisions dépend, en fin de compte, des gouvernements, et l'UICN ne peut que donner des conseils et encourager. Il existe un certain nombre d'outils pour identifier la meilleure catégorie pour un site donné. Les questions porteront parfois sur toute une série de sites similaires : par exemple, si un département des forêts essaie de décider lesquelles de ses réserves forestières devraient être reconnues comme aires protégées; ou lorsque des aires protégées privées 
Figure 3. Processus pour assigner des catégories aux aires protégées

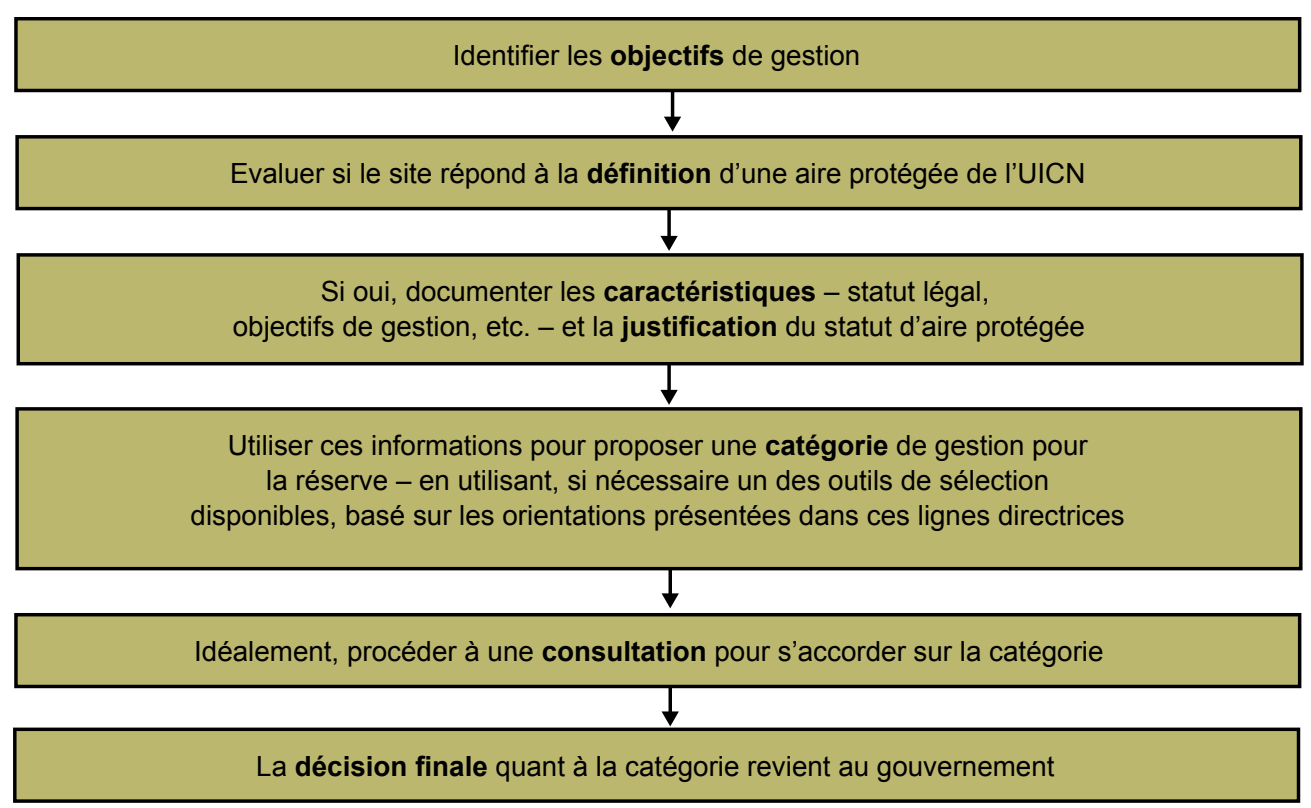

tentent d'obtenir leur reconnaissance comme aires protégées au sein du système national ; ou encore lorsque des communautés se montrent intéressées à convertir leurs zones de pêche contrôlée en aires protégées.

\section{Faire rapport}

Une fois que la catégorie est assignée, les gouvernements sont tenus d'en faire rapport au Centre mondial de surveillance continue de la conservation de la nature du PNUE pour que l'information puisse être intégrée dans la Base de données mondiale sur les aires protégées et dans la Liste des $N U$ des aires protégées. La déclaration est volontaire, mais elle est requise par un certain nombre de résolutions et de politiques de Nations unies, tout récemment encore dans le Programme de travail sur les aires protégées de la CDB. Cela signifie que l'on attend des gouvernements qu'ils rapportent les informations de façon régulière et précise, suivant le modèle fourni par le WCMC/PNUE. Des obligations semblables pèsent sur le WCMC/PNUE pour garantir que les informations soient transmises à la base de données avec exactitude et rapidité.

\section{Consolidation de l'assignation des catégories}

Lassignation des catégories relève traditionnellement de l'autorité des gouvernements, et l'on suppose qu'ils ne vont pas, pas plus que d'autres, attribuer sciemment une catégorie incorrecte et qu'ils sont à même d'assigner correctement les catégories. Cette relation fait depuis quelques années l'objet d'un examen plus approfondi et elle est remise en question particulièrement par certains groupes industriels qui sont préoccupés par la surface croissante de terres et d'eau "bloquée " pour d'autres formes de développement, mais aussi par les communautés locales inquiètes de la perte de leurs droits d'accès et autres. Certains gouvernements ont aussi demandé, pour clarification, si une aire protégée s'était bien vu assigner la bonne catégorie, en particulier là où le niveau de financement des aires protégées est fixé sur la base de la désignation de la catégorie. Il s'est dit qu'une certaine forme de procédure de doléances ou de processus de vérification pourrait être utile pour apporter une garantie indépendante que : (1) l'aire est vraiment une aire protégée ; et (2) c'est bien la catégorie appropriée qui a été assignée. En fin de compte, le choix de la catégorie revient aux gouvernements, et l'UICN n'a pas le droit ni le souhait d'imposer ce qui doit rester une décision nationale. Cependant, l'idée que l'UICN propose aux gouvernements et aux autres un cadre pour consolider et, au besoin, remettre en cause l'assignation d'une catégorie a reçu un soutien très clair.

Une option serait, pour l'UICN ou une tierce partie, d'établir un processus de certification ou de vérification visant à examiner l'assignation des catégories - ces questions sont envisagées plus en détail dans la section sur l'efficacité de gestion, principalement sous forme de cas particuliers où la vérification des normes pourrait être utile pour les propriétaires ou les gestionnaires de l'aire eux-mêmes.

Un problème différent concerne la possibilité que des parties prenantes extérieures contestent l'assignation d'une catégorie. Il est à espérer que ces cas restent rares, mais il est de plus en plus clair qu'il faut, au sein de l'UICN et de la CMAP, un système pour traiter ces cas. La CMAP/UICN a l'intention de 
coopérer avec ses partenaires, y compris le WCMC/PNUE, afin d'étudier les options pratiques pour mettre en place l'une ou l'autre forme de procédure de doléances dans un avenir proche.

Une telle procédure ne pourra jamais être que symbolique : ce sont les gouvernements qui ont le droit ultime de dire comment une aire protégée est gérée et comment elle est classée. Mais des évaluations indépendantes de ce type se sont avérées très importantes du point de vue politique dans des situations semblables, comme le Registre de Montreux de Ramsar et le mécanisme de Suivi réactif de la Convention du patrimoine mondial.

L'UICN reconnaît le besoin d'aider les gouvernements et les autres institutions à accroître leurs capacités en termes de compréhension et d'application des catégories. En conjonction avec le lancement des nouvelles lignes directrices sur les catégories, l'UICN prépare un projet majeur sur le renforcement des capacités concernant leur application. 


\section{Utiliser les catégories}

Les catégories ont été conçues au départ comme un moyen de classer et d'enregistrer les aires protégées ce qui est déjà une tâche très ardue. Graduellement, de nouvelles utilisations se sont ajoutées, y compris, en particulier, un rôle dans la planification de systèmes d'aires protégées et dans le développement de politiques de conservation cohérentes : après une réticence initiale, les membres de I'UICN eux-mêmes ont approuvé cette approche en recommandant que les gouvernements interdisent l'exploitation minière dans les aires protégées des catégories I à IV. 


\section{Utiliser les catégories de l'UICN comme un instrument de planification de la conservation}

De tout temps, les catégories de gestion des aires protégées ont été utilisées par des organismes de gestion pour classer, avec une précision plus ou moins grande, la raison d'être d'une aire protégée donnée, une fois que celle-ci avait été déterminée lors de sa planification. L'UICN recommande que les catégories de gestion soient également utilisées pour aider lors de la conception de systèmes d'aires protégées dotées de divers objectifs de gestion (et types de gouvernance), pour leur permettre de répondre aux besoins de la biodiversité dans tout le paysage terrestre ou marin. Alors que l'on demande aux gouvernements d'identifier et de combler les lacunes de leur système d'aires protégées, les planificateurs doivent considérer la gamme complète des catégories de gestion d'aires protégées lorsqu'ils identifient, conçoivent et lancent la gestion de nouvelles aires protégées.

\section{Contexte}

Alors que l'utilisation et la consommation humaines dominent les paysages terrestres et marins du monde entier, il est de plus en plus nécessaire de considérer les aires protégées comme un éventail de pratiques de gestion plutôt que comme des endroits isolés, verrouillés et réglementés. L'approche " taille unique " de la gestion de la biodiversité dans les aires protégées n'est pas seulement source de conflits avec les autres besoins sociétaux, mais elle limite aussi les options de gestion pour les conservationnistes et la quantité de terres et d'eau disponible pour la protection de la biodiversité. La diversité des catégories d'aires protégées peut servir à s'attaquer aux besoins écologiques touchant une espèce ou un écosystème, et à l'équilibrer par rapport aux besoins de la société.

Selon les accords de la CDB, les gouvernements s'engagent à compléter des systèmes d'aires protégées écologiquement représentatifs, et ce processus commence d'habitude par l'identification des lacunes dans le système existant - généralement par une analyse écologique des lacunes. Dans le contexte de la conservation, l'analyse des lacunes est une méthode pour identifier la biodiversité $(c .-\grave{a}-d$. des espèces, des écosystèmes et des processus écologiques) qui n'est pas suffisamment préservée dans un système d'aires protégées ou par d'autres mesures de conservation efficaces et à long terme. Des analyses des lacunes bien conçues identifient trois types de lacunes dans un système d'aires protégées (Dudley et Parrish 2006) :

- Lacunes de représentativité : pas de couverture, ou couverture insuffisante pour une espèce ou un écosystème dans les aires protégées ;

- Lacunes écologiques : le système d'aires protégées ne parvient pas à contenir des lieux ou des phénomènes clés pour la conservation d'une espèce ou d'un écosystème pendant toute la durée du cycle vital ;

- Lacunes de gestion : les aires protégées couvrent géographiquement les éléments de la biodiversité, mais n'arrivent pas à les protéger à cause d'une gestion insuffisante ou inappropriée.

Lorsque les lacunes sont identifiées et que les actions réactives sont effectuées - de nouvelles aires protégées sont proposées et l'on réalise une révision des catégories de gestion pour les aires protégées existantes - toute la gamme des catégories de gestion doit être considérée.

Lorsque l'on passe en revue les catégories des aires protégées existantes pour déterminer le type de protection qui préservera le mieux la biodiversité dans l'aire donnée, il n'y a aucune hiérarchie qui permettrait de dire, par exemple, qu'une aire protégée de catégorie I est toujours meilleure qu'une catégorie II ou III ou IV. D'autre part, les catégories ne sont pas simplement interchangeables. Le seul principe qui doit s'appliquer en assignant une catégorie, c'est l'adéquation de la raison d'être de la gestion assignée à une aire protégée au sein du système, par rapport aux besoins écologiques de, et aux menaces sur, les espèces ou l'écosystème dans le contexte de tout le paysage, terrestre ou marin, où se trouve cette biodiversité. Les objectifs de l'aire protégée doivent aussi être examinés au moment de revoir et d'assigner une catégorie de gestion. Dans certains cas, il peut être préférable de durcir la protection en raison du déclin du statut écologique ou de conservation d'une espèce ou d'un écosystème à l'intérieur de l'aire protégée ou dans toute son aire de répartition - p.ex. une partie ou toute une aire protégée de catégorie $\mathrm{V}$ pourrait se voir réassigner la catégorie Ib. Dans d'autres, il peut être plus stratégique de modifier la gestion pour permettre plus de flexibilité en termes d'utilisation durable (p.ex. une aire protégée qui passe de la catégorie II à la catégorie VI).

Augmenter la rigueur de la protection sera normalement une réponse à un déclin persistant de la biodiversité dans une aire protégée existante. Quand des gestionnaires de ressources naturelles peuvent-ils choisir une approche moins stricte de la protection d'une aire plutôt qu'une plus stricte ? Les exemples comprennent :

- Quand la viabilité de la population d'une espèce ou l'intégrité d'un écosystème s'est améliorée au travers de toute sa distribution et qu'elle n'exige plus une utilisation humaine réduite et une protection intense.

- Quand les utilisations humaines potentielles dans une catégorie d'aire moins protégée risquent peu d'affecter la santé de l'espèce ou de l'écosystème.

- Lorsqu'un changement de catégorie augmente la superficie de l'aire protégée au bénéfice des espèces et des écosystèmes cibles. Par exemple, quand il s'agit de la protection d'une rivière ou d'eau douce, il peut être plus efficace de 
gérer une plus grande portion du bassin versant pour ses fonctions écosystémiques, avec une protection moins restrictive, que de protéger le cours principal de la rivière par une catégorie I ou II, en fonction des menaces prioritaires qui pèsent sur la cible biologique.

- Lorsque la biodiversité s'est adaptée à des systèmes de gestion culturels et que l'absence de ces interventions exerce désormais une pression sur la survie ou la viabilité de l'espèce.

\section{Quelques réflexions avant d'assigner les catégories de gestion des aires protégées dans la planification d'un système d'aires protégées}

Il n'existe aucune règle ferme et définitive pour attribuer une catégorie particulière à une aire protégée donnée. Cependant, l'approche primordiale doit consister à reconnaître que toutes les aires protégées ne seront pas gérées de la même façon et que le choix des approches de gestion doit se faire en pesant les différentes opportunités et pressions relatives à l'aire. Certains principes généraux sont présentés ci-dessous.

- Commencer par les besoins écologiques des espèces et des écosystèmes. Les options de gestion doivent être déterminées d'abord par les caractéristiques écologiques et par le passé des espèces et des écosystèmes. Par exemple, des espèces différentes répondent différemment aux perturbations et, en général, l'espèce la plus sensible peut avoir besoin d'une protection plus stricte par une approche de gestion plus restrictive.

- Examiner les menaces sur les espèces ou les valeurs de l'écosystème. Certaines approches de gestion se prêtent bien au traitement de certaines menaces. Par exemple, le braconnage dans les aires marines protégées est mieux pris en charge si l'on permet aux communautés locales de pêcheurs d'y accéder, avec un taux de prélèvement convenu (p.ex. dans une aire protégée de catégorie V ou VI), ce qui les encourage à contrôler le braconnage externe.

- Examiner les objectifs de l'aire protégée, les désignations internationales existantes et proposées, et voir comment ils contribuent aux efforts de conservation de la biodiversité au niveau du paysage, du pays et du monde entier. Chaque aire protégée existante doit avoir été créée pour des raisons spécifiques. Mais lorsque l'approche de planification est élargie pour appréhender le niveau du paysage ou du pays, il peut être nécessaire de reconsidérer les raisons d'être et les objectifs originaux. Les désignations internationales comme celles de la Convention du patrimoine mondial ou celle de Ramsar sont utiles pour identifier la meilleure approche pour gérer un site.

- Envisager de développer et de mettre en œuvre un processus pour assigner/revoir les catégories de gestion dans un pays. Une agence nationale des aires protégées doit mettre au point une procédure officielle pour revoir et assigner des catégories de gestion. Par exemple, suite à une évaluation écologique de ses lacunes, l'agence en charge des aires protégées à Panama a révisé les catégories de gestion de toutes les aires protégées du pays.

- Pas de perte du caractère naturel, d'une fonction écosystémique ou de viabilité d'une espèce. L'option de gestion choisie ne doit pas, dans la plupart des cas, aboutir à la perte du caractère naturel actuel dans l'aire protégée (p.ex. l'UICN ne propose pas, d'habitude, une catégorie $\mathrm{V}$ ou VI dans un site plus ou moins naturel), mais il peut y avoir des exceptions.

- Considérer l'ensemble du paysage terrestre ou marin lorsque l'on assigne les catégories. Le choix de la catégorie doit refléter la contribution de l'aire protégée à la mosaïque globale de la conservation plutôt qu'uniquement les valeurs du site en question, c.-à-d. que les objectifs de gestion pour tout site donné ne doivent pas être choisis en fonction de lui seul. Par exemple, un lac intérieur peut ne pas être important seulement pour les populations résidentes mais aussi comme étape pour les oiseaux migrateurs. De même, nous recommandons que les planificateurs environnementaux développent une gamme variée d'aires protégées de diverses catégories de l'UICN pour un élément donné de la biodiversité.

- Les parties prenantes sont importantes. Les options en matière de gestion doivent envisager les besoins, les capacités et les souhaits des communautés locales et doivent généralement être sélectionnées après discussion avec les parties prenantes - les objectifs de gestion qui ont le soutien des communautés locales sont plus susceptibles de réussir que celles qui sont impopulaires ou combattues.

- Penser à l'efficacité de gestion en assignant les catégories d'aires protégées. Les gestionnaires doivent aussi tenir compte de l'efficacité de gestion existante et probable dans une aire donnée lorsqu'ils recommandent un objectif de gestion principal (catégories d'aire protégée). Une gestion inefficace dans une aire de catégorie I ou II (le syndrome du parc sur papier) peut avoir un impact de conservation moindre qu'une aire protégée de catégorie $\mathrm{V}$ ou VI efficace, même si les règles de la gestion dans ces dernières sont moins strictes.

- Les catégories de gestion plus strictes ne sont pas toujours meilleures. Les scientifiques de la conservation supposent souvent que les catégories I à IV signifient une conservation plus efficace que les catégories V-VI lorsqu'ils assignent une catégorie à des aires protégées. Ce n'est pas toujours le cas : par exemple, des approches moins restrictives qui couvrent des superficies plus étendues peuvent parfois être plus efficaces.

- Utiliser les catégories comme un instrument de planification interne à l'aire protégée. A l'intérieur d'une aire protégée, on peut accepter plusieurs zones avec des objectifs de gestion différents si cela aide à la gestion générale. 
On peut envisager des zones temporaires dans des aires protégées (p.ex. pour permettre l'exploitation durable à faible impact de produits forestiers non ligneux par les communautés locales).

- Considérer les bénéfices sociétaux de la diversification de la gamme des catégories. En envisageant toute une variété de catégories de gestion d'aires protégées, on peut améliorer la perception des aires protégées par le public et augmenter les chances de succès - particulièrement si les gens reconnaissent que toutes les aires protégées ne signifient pas que les ressources terrestres, aquatiques ou marines sont "bloquées ". L'emploi de certaines catégories peut susciter l'engagement des parties prenantes envers la conservation et élargir les options de désignation d'aires pour la protection (p.ex. les sites sacrés pour la religion des populations locales qui représentent aussi des contributions importantes à la biodiversité, comme dans le cas du Parc National de Tikal, au Guatemala).

\section{Prévoir les changements climatiques}

Le réchauffement mondial va affecter la planification des aires protégées de nombreuses façons. Les changements climatiques vont entraîner une augmentation de la température annuelle moyenne, du changement dans le régime des eaux et, presque certainement, une plus faible prédictibilité. Il est probable qu'il y aura des changements majeurs dans les attributs naturels propres aux écosystèmes et aux habitats et dans la répartition des caractéristiques biotiques naturelles. Les zones humides pourraient s'assécher dans certaines régions du monde, alors qu'ailleurs des aires arides pourraient être soumises à des inondations. Les îles et les terres côtières les plus basses seront plus sensibles à l'érosion et à la disparition de sols et d'habitats suite à la montée du niveau des océans et à des conditions plus tumultueuses. Les espèces et les habitats qui subsistent à la limite de leur distribution géographique sont plus susceptibles d'être affectés par les changements climatiques mondiaux. Les rythmes saisonniers des plantes et des animaux vont aussi se modifier. Il est probable que de nombreuses aires protégées seront touchées, perdant peut-être des espèces et des écosystèmes ; d'autres espèces pourraient venir prendre leur place, mais il est probable que de nombreuses espèces, moins mobiles et moins adaptables, feront face à des menaces d'extinction. Mais dans le même temps, les aires protégées pourront jouer un rôle dans l'atténuation des changements climatiques, en constituant des tampons contre les événements climatiques extrêmes (Stolton et al. 2008) ainsi qu'un réseau d'habitats naturels fournissant autant de voies pour des migrations rapides et d'espaces où l'évolution et l'adaptation pourront se faire (Dudley et Stolton 2003).

Les gestionnaires et les autorités des aires protégées commencent à examiner les options possibles pour réduire l'impact que les changements climatiques auront sur les aires protégées et pour optimiser les avantages que des systèmes d'aires protégées bien conçus peuvent avoir pour la société en atténuant ces impacts. En matière d'objectifs et de catégories de gestion, ceci comporte un certain nombre d'implications :

- Les impacts probables des changements climatiques devraient être pris en compte lors de la conception des systèmes d'aires protégées afin d'optimiser les opportunités offertes par toute une gamme d'approches de gestion, fondées sur une bonne appréhension des forces et des faiblesses des différentes catégories face aux changements climatiques. Ceux-ci doivent être pris en compte aujourd'hui dans la planification de systèmes d'aires protégées et celle d'aires protégées individuelles, pour être prêts pour les changements du futur (en gardant à l'esprit que nous ne savons pas encore avec la moindre certitude, le plus souvent, ce que seront ces changements - la planification doit donc se montrer flexible).

- L'interconnexion d'aires protégées par des corridors et des réseaux va s'avérer encore plus essentielle pour faciliter les déplacements des espèces et accroître la probabilité de transferts naturels vers des endroits où les conditions seront plus favorables à leur survie. Là où ce sera possible, il sera très approprié de concevoir de plus vastes aires protégées contenant une plus large gamme de caractéristiques biogéographiques.

- Certaines espèces seront confrontées à une extinction totale s'il n'y a pas, dans l'espace où leur expansion naturelle est possible, des lieux où le régime climatique convient à leur survie. Il peut donc être nécessaire de développer des plans pour la translocation d'espèces vers des endroits plus appropriés et pour améliorer les liens entre les efforts de conservation in-situ et ex-situ.

- Les changements climatiques signifieront probablement davantage de gestion interventionniste pour protéger l'existence d'espèces et d'habitats. Cela va susciter des questions quant à l'assignation de la catégorie, et peutêtre un plus grand recours à des approches de type catégorie IV.

- Les conditions changeantes peuvent impliquer aussi des altérations de la gestion au sein des aires protégées ellesmêmes. Dans certains cas, des conditions plus rudes pourraient fragiliser les paysages culturels traditionnels et aussi menacer les espèces subsistantes, ce qui nécessiterait le changement de la catégorie $\mathrm{V}$ vers, disons, la catégorie Ib, associée à l'abandon par les hommes. Dans d'autres situations, ce sont des environnements autrefois à peu près intacts qui ne pourront plus subsister que grâce à l'intervention humaine, et une approche de type catégorie Ia pourrait devoir passer à la catégorie IV. Il faut noter que les changements de catégorie devraient rester des événements rares qui feront l'objet d'une procédure aussi rigoureuse que l'assignation originale. 


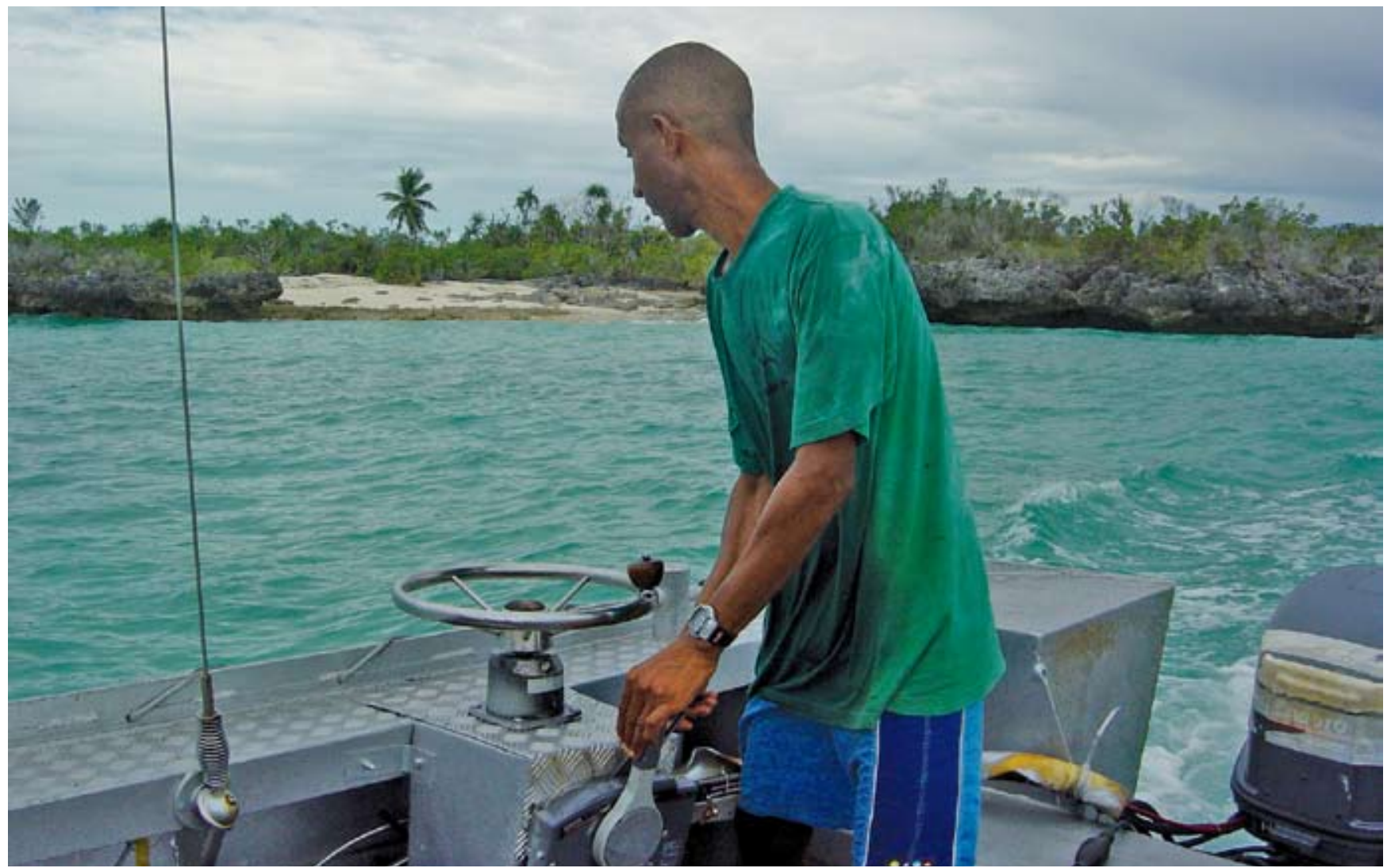

Aux Seychelles, l'Atoll d'Aldabra, de catégorie Ia, est isolé à l'ouest de l'océan Indien. Il constitue un laboratoire naturel idéal pour étudier des écosystèmes marins tropicaux et les environnements qui y sont liés (comme les prairies marines et les mangroves). (C) Sue Stolton

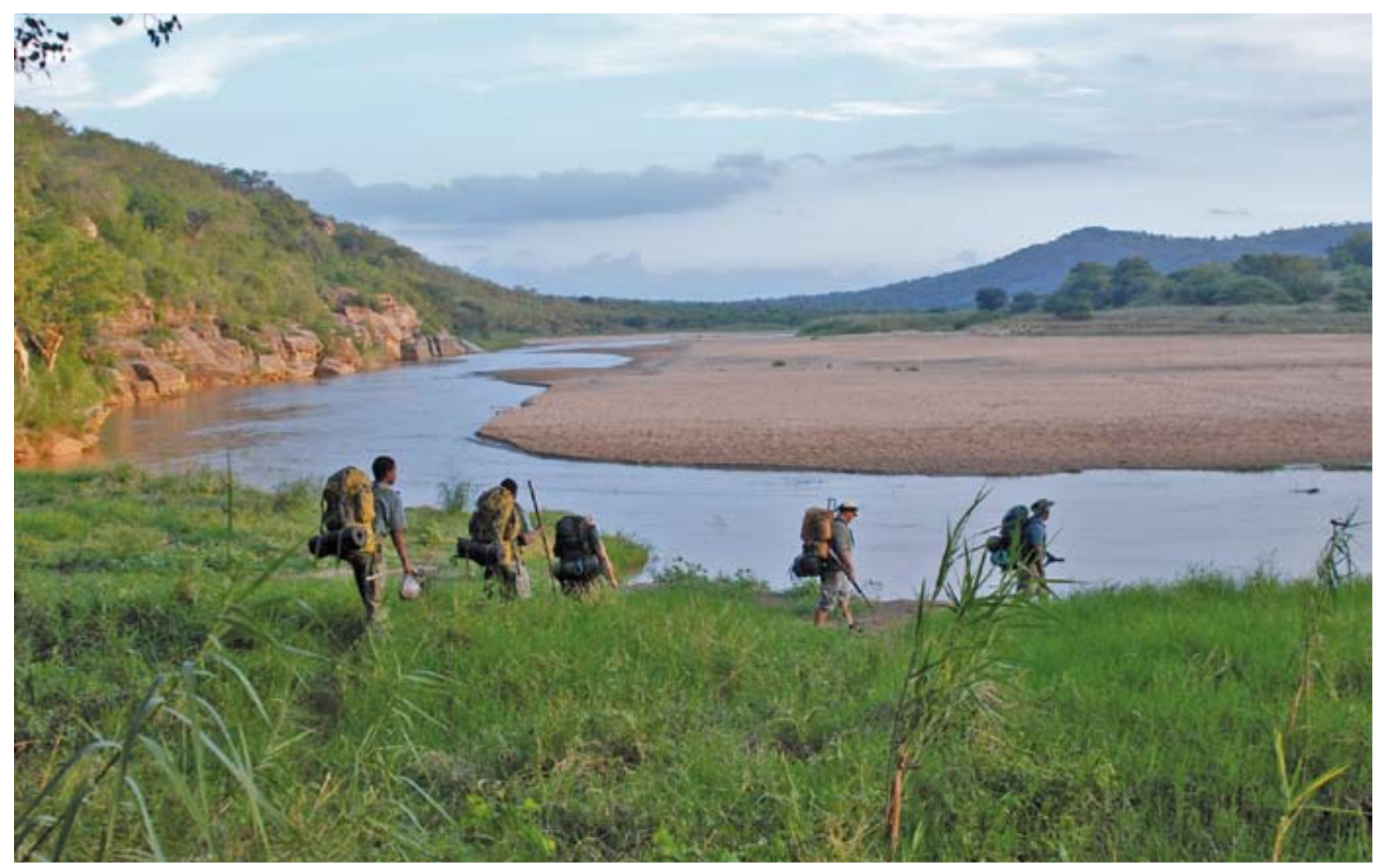

L'Imfolozi Wilderness Area (dans la Réserve de Faune d'Imfolozi), au KwaZulu Natal, Afrique du Sud, est une aire de catégorie 1b gérée par la Province, d'où est venue l'impulsion de créer d'autres zones de nature sauvage en Afrique. Ici, des " pisteurs " avec la Wilderness Leadership School visitent l'aire pour une promenade de cinq jours qui utilise des pratiques de campement à faible impact. (C) Vance G. Martin 


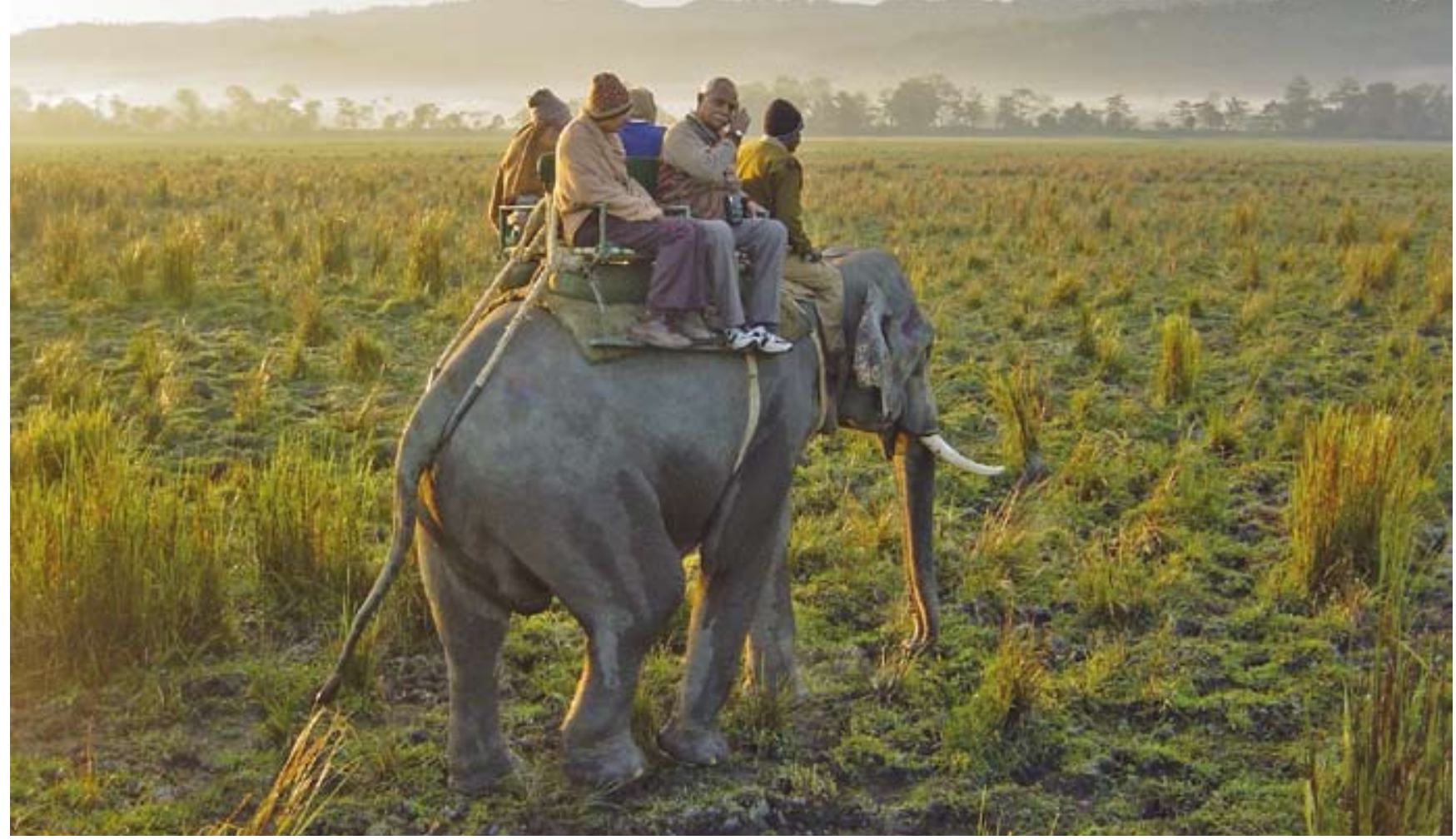

Kaziranga est un parc national classique de catégorie II. Fameux pour son grand rhinocéros unicorne de l'Inde, le paysage de Kaziranga peut être apprécié par les touristes lors de balades à dos d'éléphant ou en bateau sur le Brahmapoutre. (C) Nigel Dudley

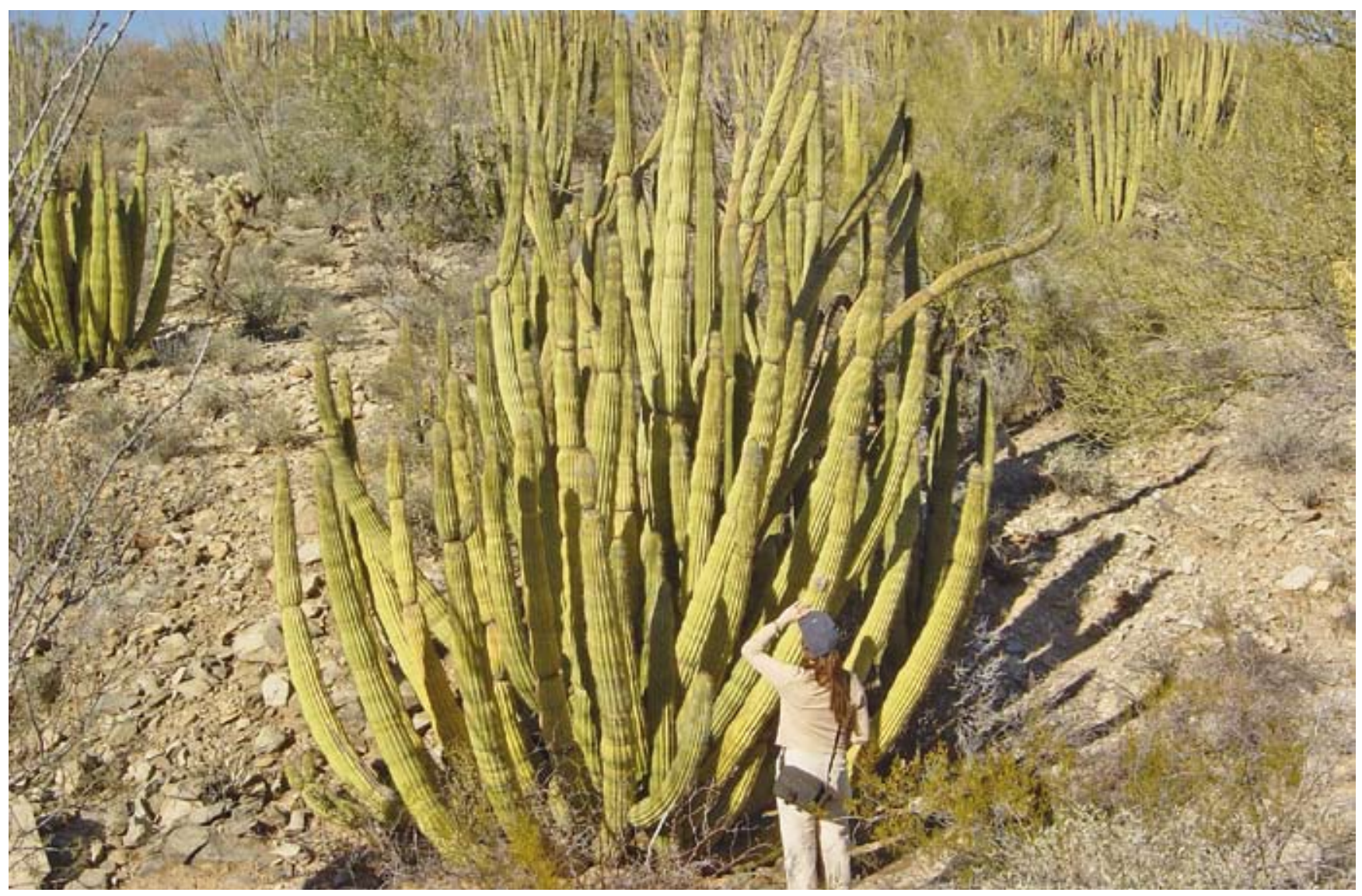

L'Organ Pipe Cactus National Monument (catégorie III) protège la majorité des cactus “tuyaux d'orgues” que l'on trouve aux Etats-Unis.

(C) Nigel Dudley 


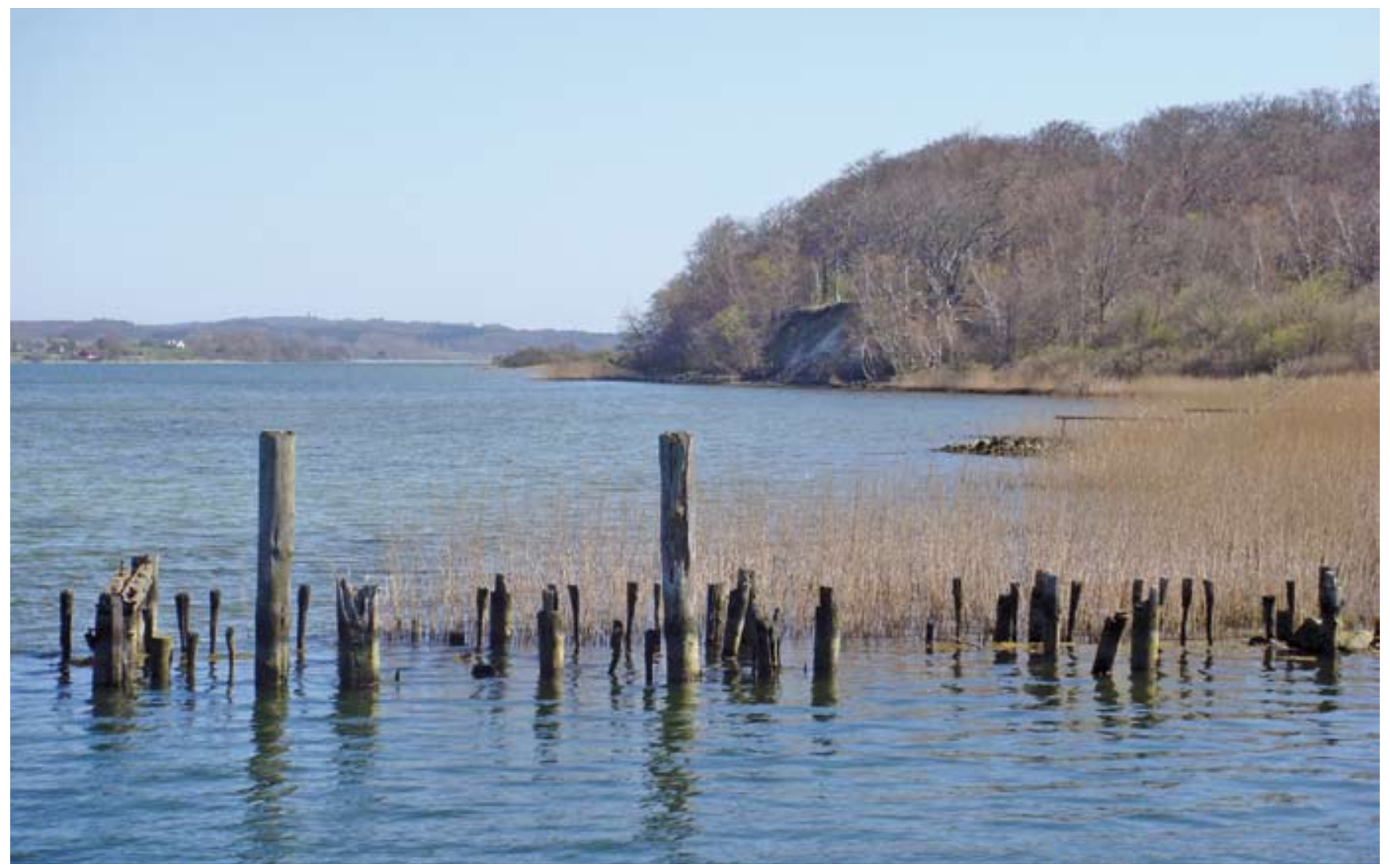

Couvrant moins d'un km², l'Insel Vilm Nature Reserve, de catégorie IV, renferme certaines des plus vieilles forêts de chênes et de hêtres d'Allemagne ; les visites sont strictement contrôlées et une grande partie de l'île est inaccessible. (C) Sue Stolton

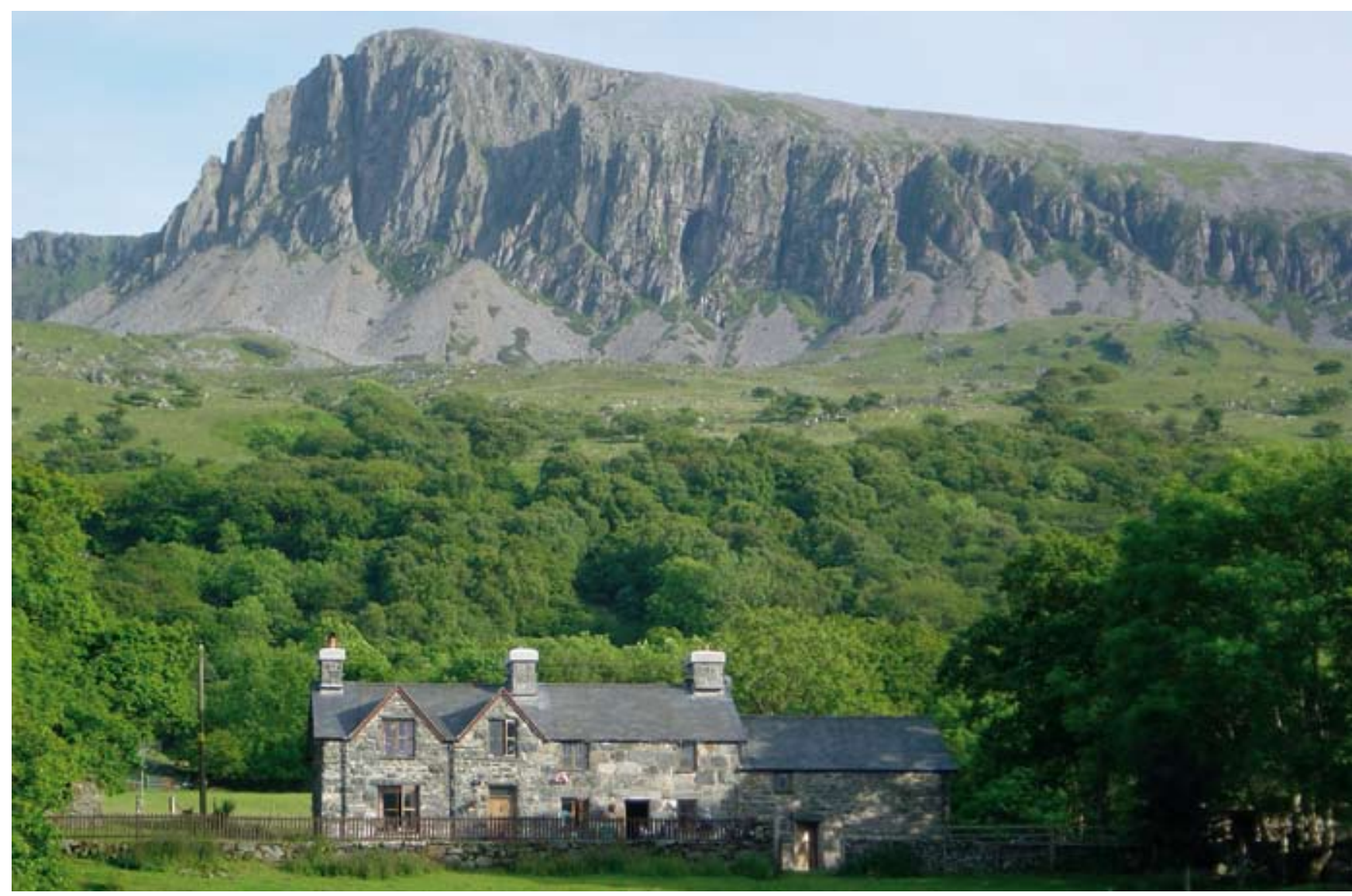

De catégorie V, le Parc National de Snowdonia, au pays de Galles, protège de grandes étendues de hauteurs battues des vents et de sommets déchiquetés dans un paysage culturel dominé par les impacts du pastoralisme et d'une ancienne industrie minière. (C) Nigel Dudley 


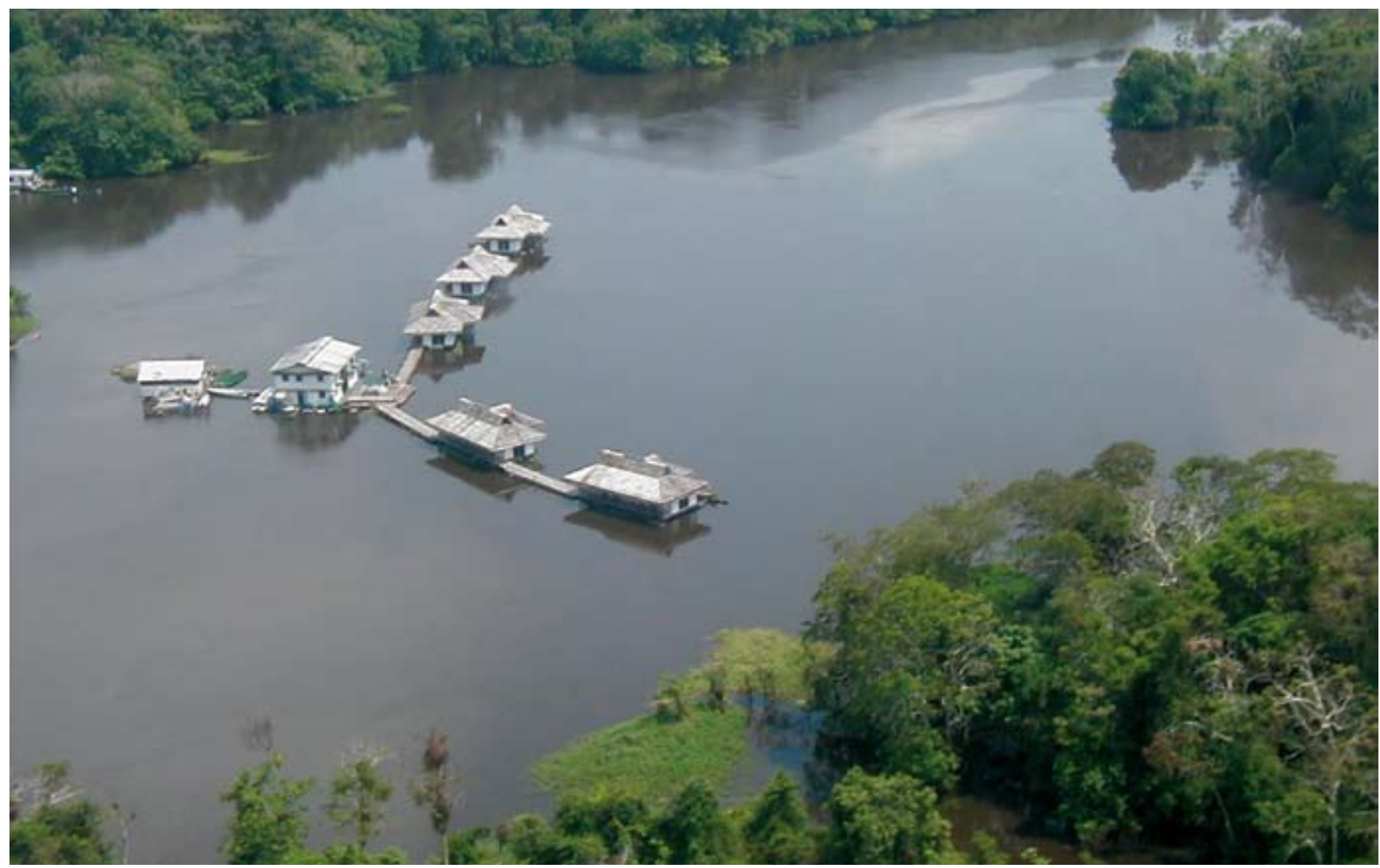

La Réserve de Développement durable de Mamirauá (catégorie VI), au Brésil, fait partie d’un grand complexe de conservation (plus de 6 millions d'ha) dans le Bassin de l'Amazone. Sa gestion concilie le besoin de préserver la biodiversité tout en fournissant des possibilités d'améliorer les moyens de subsistance durables de la population locale. (C) Jim Barborak

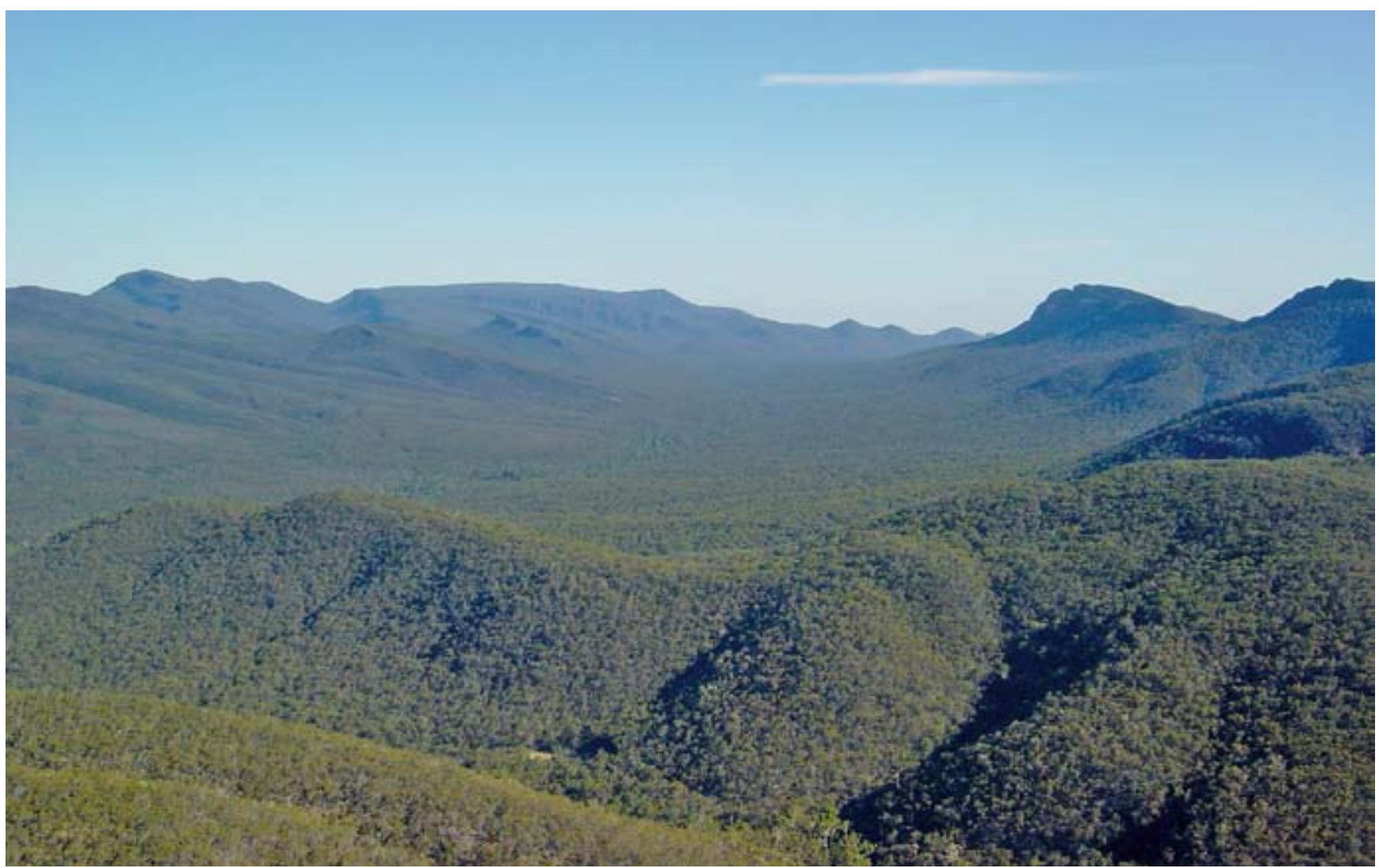

Le Parc National des Grampians (de catégorie II) à Victoria, en Australie, protège 975 espèces de plantes vasculaires, soit un tiers de la flore de l'Etat, dont 148 espèces sont menacées à Victoria. (C) Nigel Dudley 


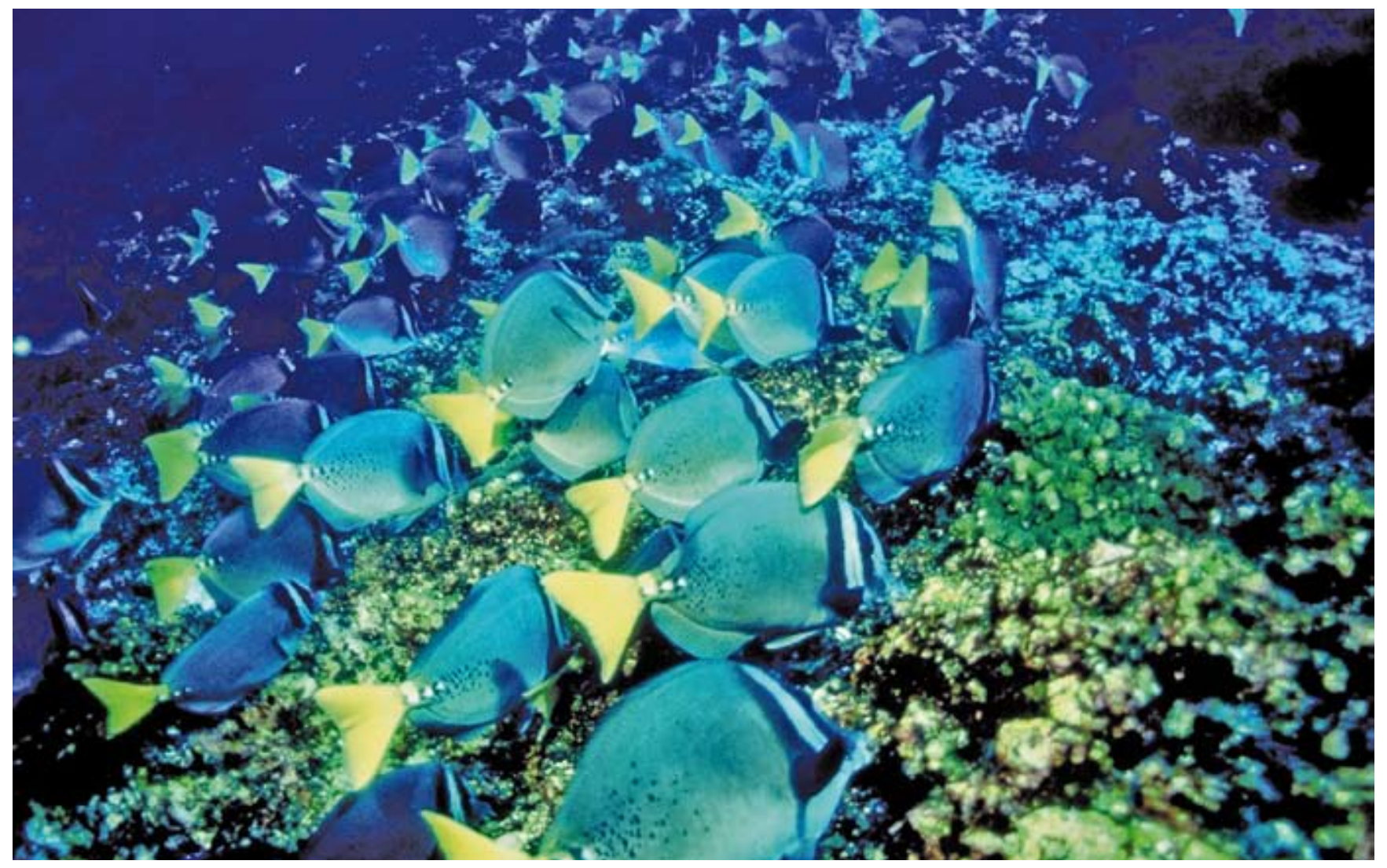

Les eaux très productives protégées par la Réserve de l'Atol das Rocas (catégorie Ia, Brésil) procure des sites d'alimentation pour des espèces telles que des thons, des poissons à rostre, des cétacés, des requins et des tortues marines, avant qu’elles migrent vers les côtes africaines de l'Atlantique. (C) Pedro Rosabal

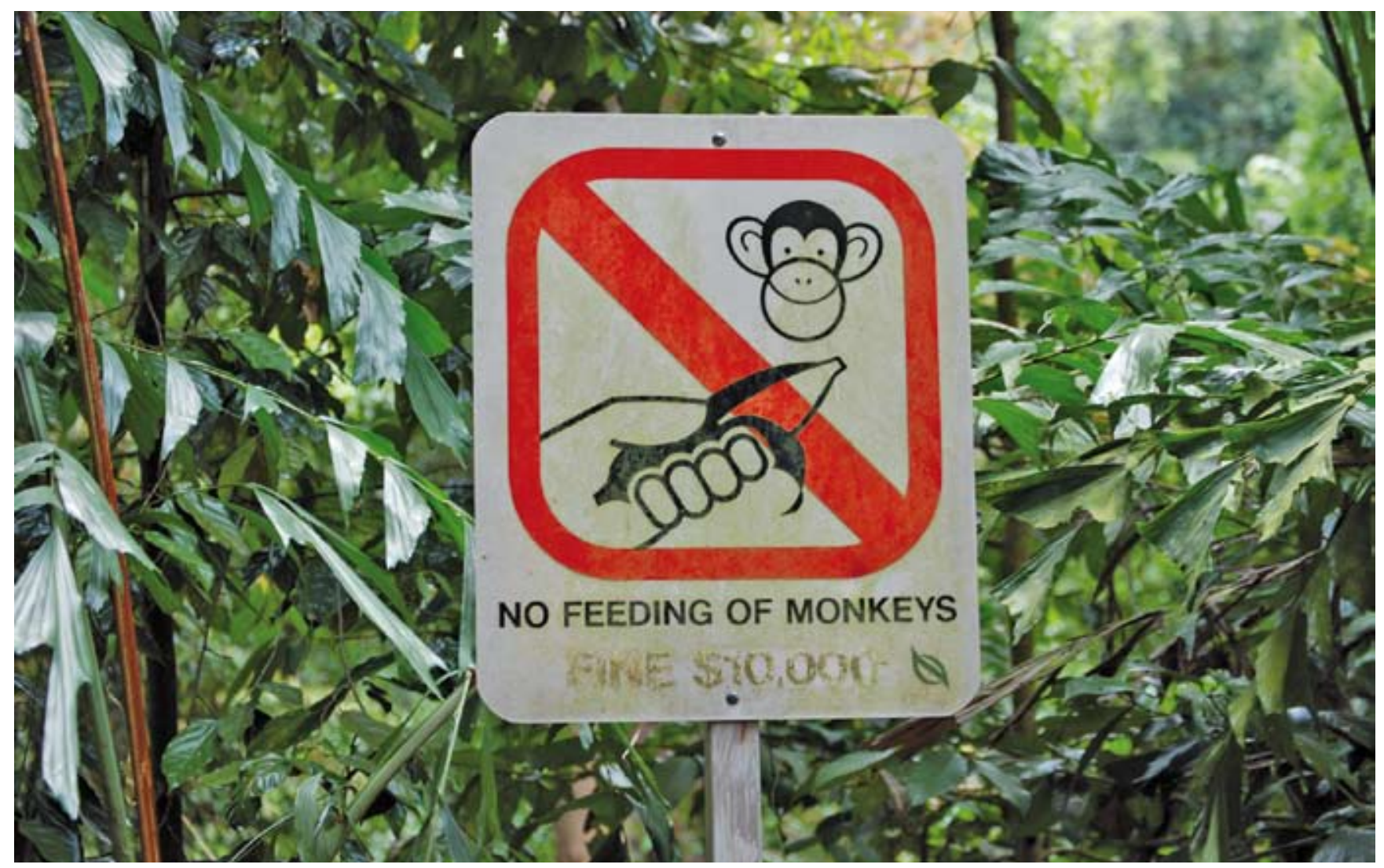

Les seules zones de forêt pluviale qui subsistent à Singapour sont protégées par la Réserve forestière de Bukit Timah (164 ha) et par celle de Central Catchment (environ 2000 ha) voisine, toutes deux aires protégées de catégorie IV. Ensemble, elles englobent moins de quatre pourcent de la forêt pluviale originale. (C) Nigel Dudley 


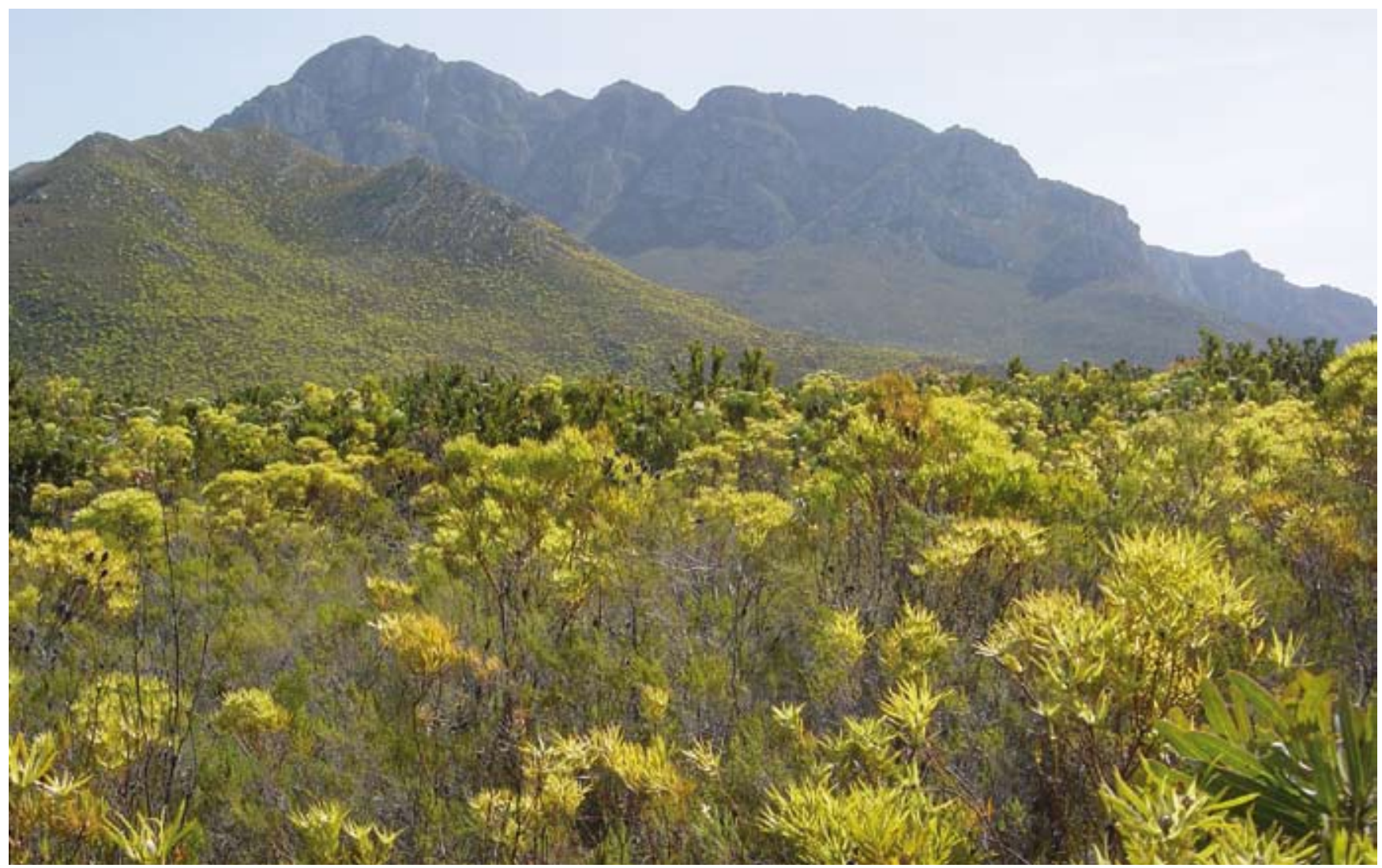

La Réserve de biosphère de Kogelberg fut la première réserve de biosphère déclarée en Afrique du Sud ; elle fait partie du réseau mondial de réserves de biosphère de l'UNESCO. La réserve peut s'enorgueillir de répertorier 1300 espèces végétales différentes sur 10.000 km², la plus forte diversité de plantes au monde. (C) Nigel Dudley

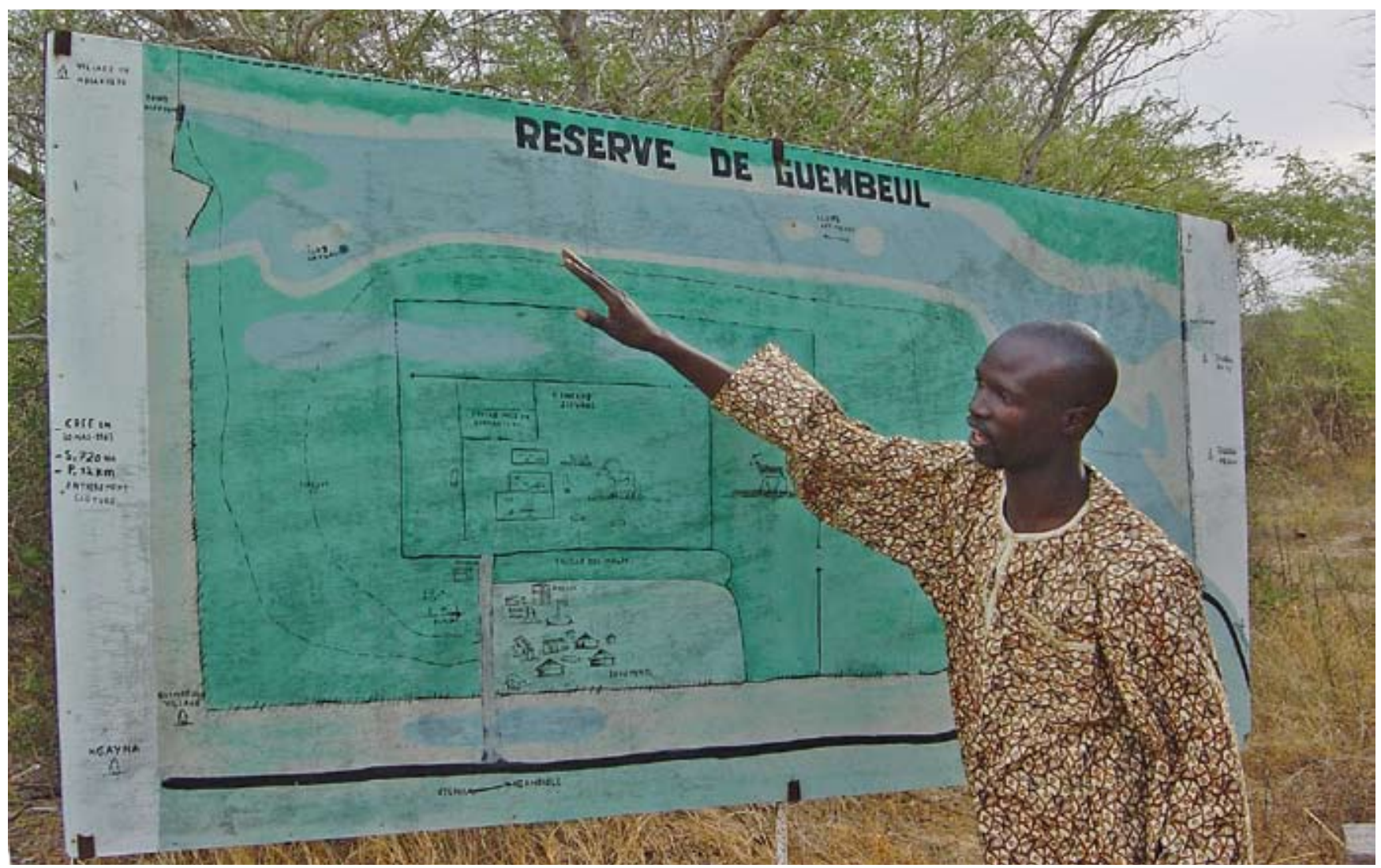

La Réserve de Geumbeul est une petite aire préservée par une communauté du Sénégal, qui protège des mangroves côtières, des populations reproductrices d'une tortue géante et le gemsbok (Oryx gazella). (C) Nigel Dudley 


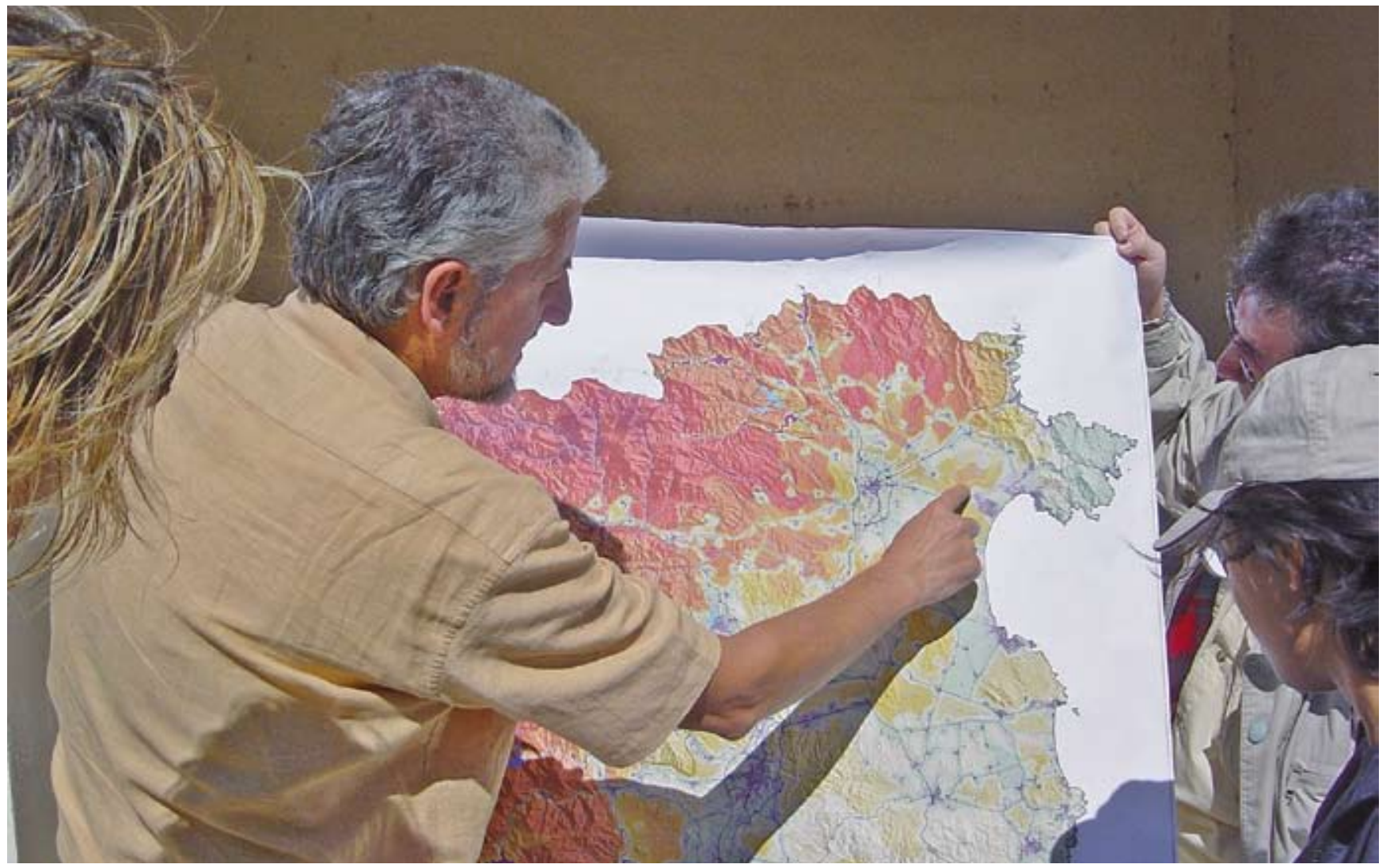

Discussion sur le zonage d'aires protégées en Catalogne, Espagne. Un système d'aires protégées de différentes catégories aide à préserver la biodiversité de ce riche paysage méditerranéen. (C) Nigel Dudley

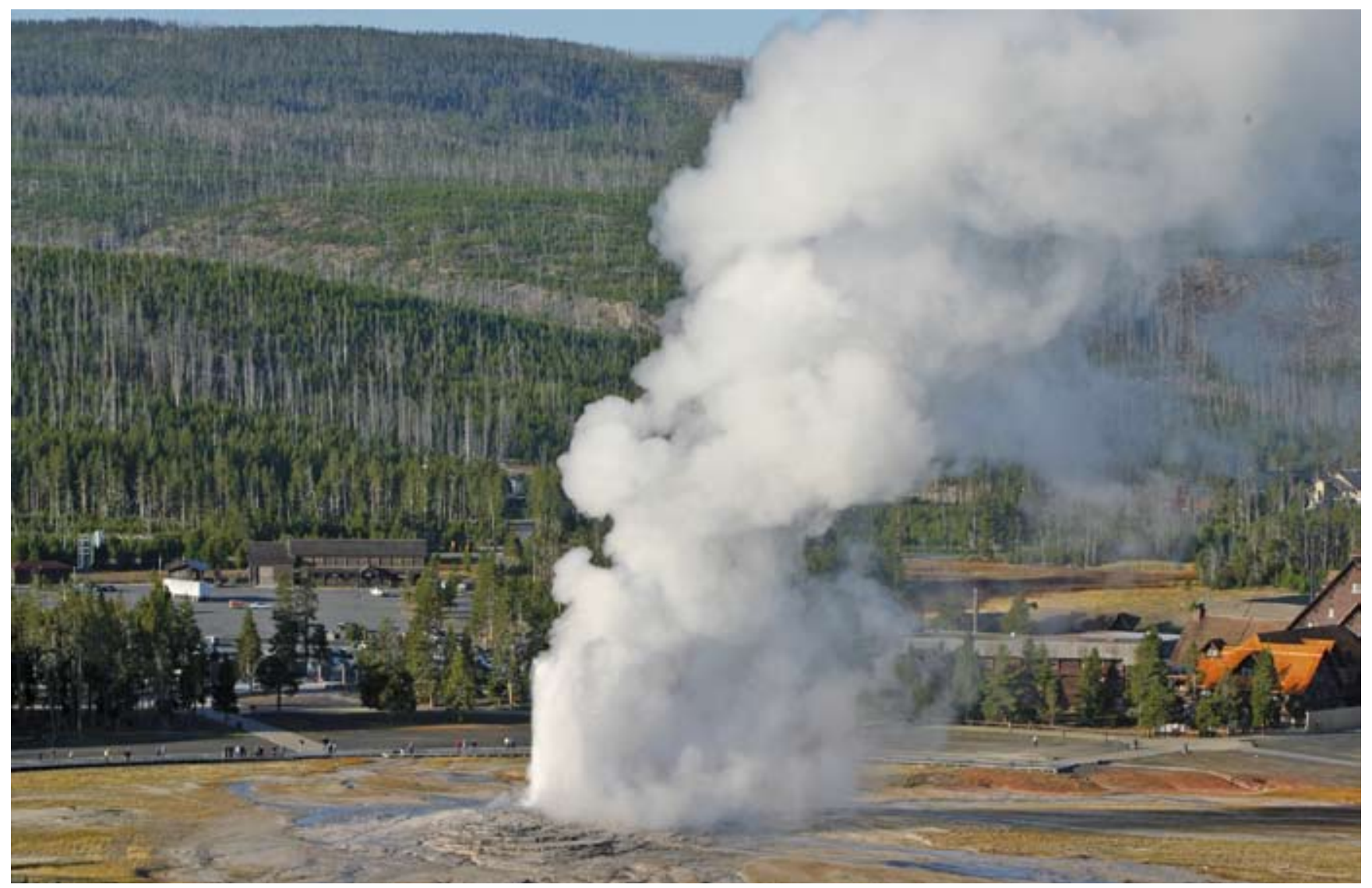

Le Parc National de Yellowstone (catégorie II) aux USA est un paysage continuellement façonné par des forces géologiques. Le Yellowstone contient la collection la plus diverse et la plus intacte de geysers, de sources d'eau chaude, de boues bouillonnantes et de fumerolles. (C) Roger Crofts 


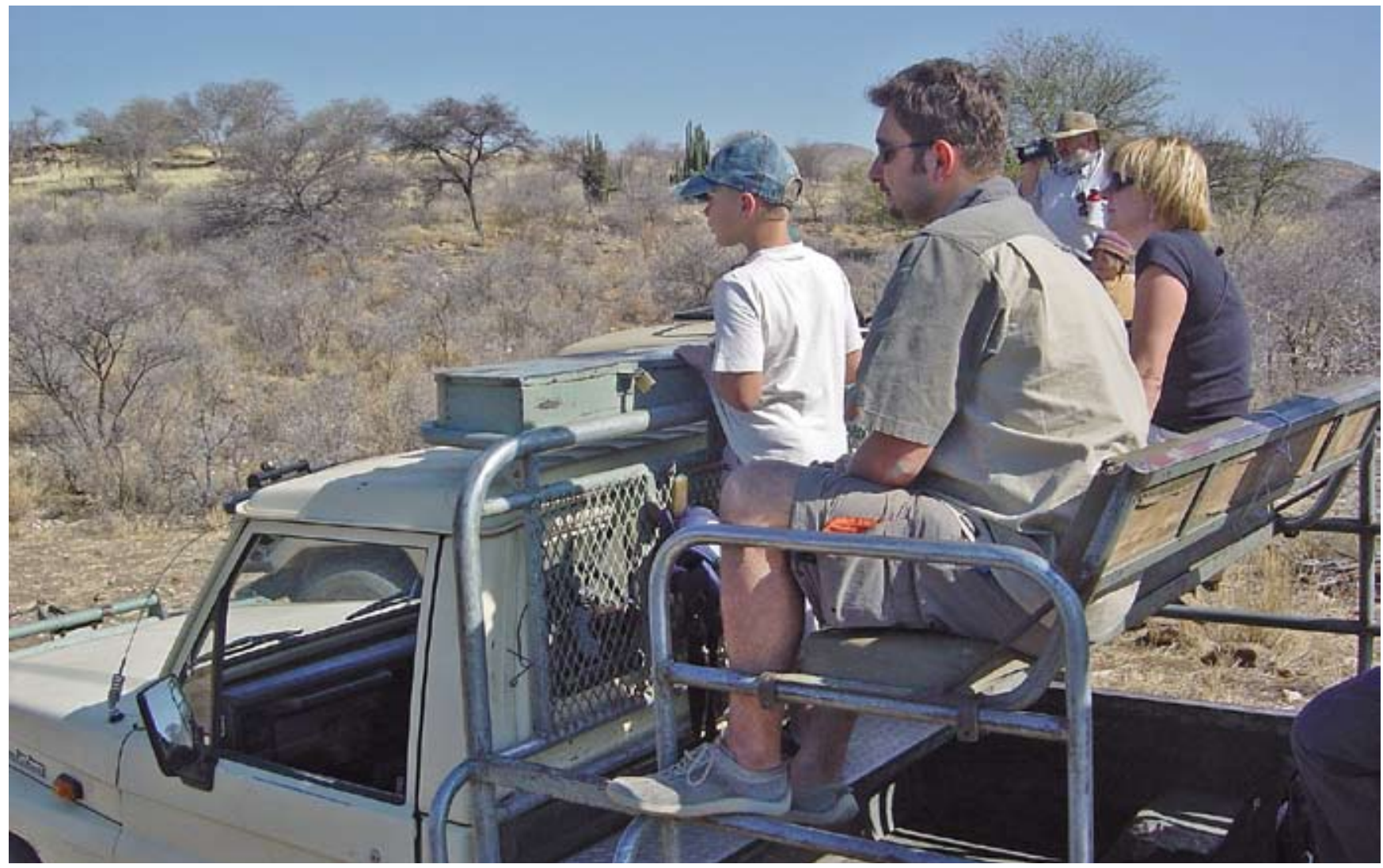

On estime qu'en Afrique australe (Botswana, Namibie, Afrique du Sud et Zimbabwe), il y a quelque 14 millions d'ha de terrains privés dont la faune sauvage bénéficie de l'une ou l'autre forme de protection ou de gestion durable. (C) Nigel Dudley

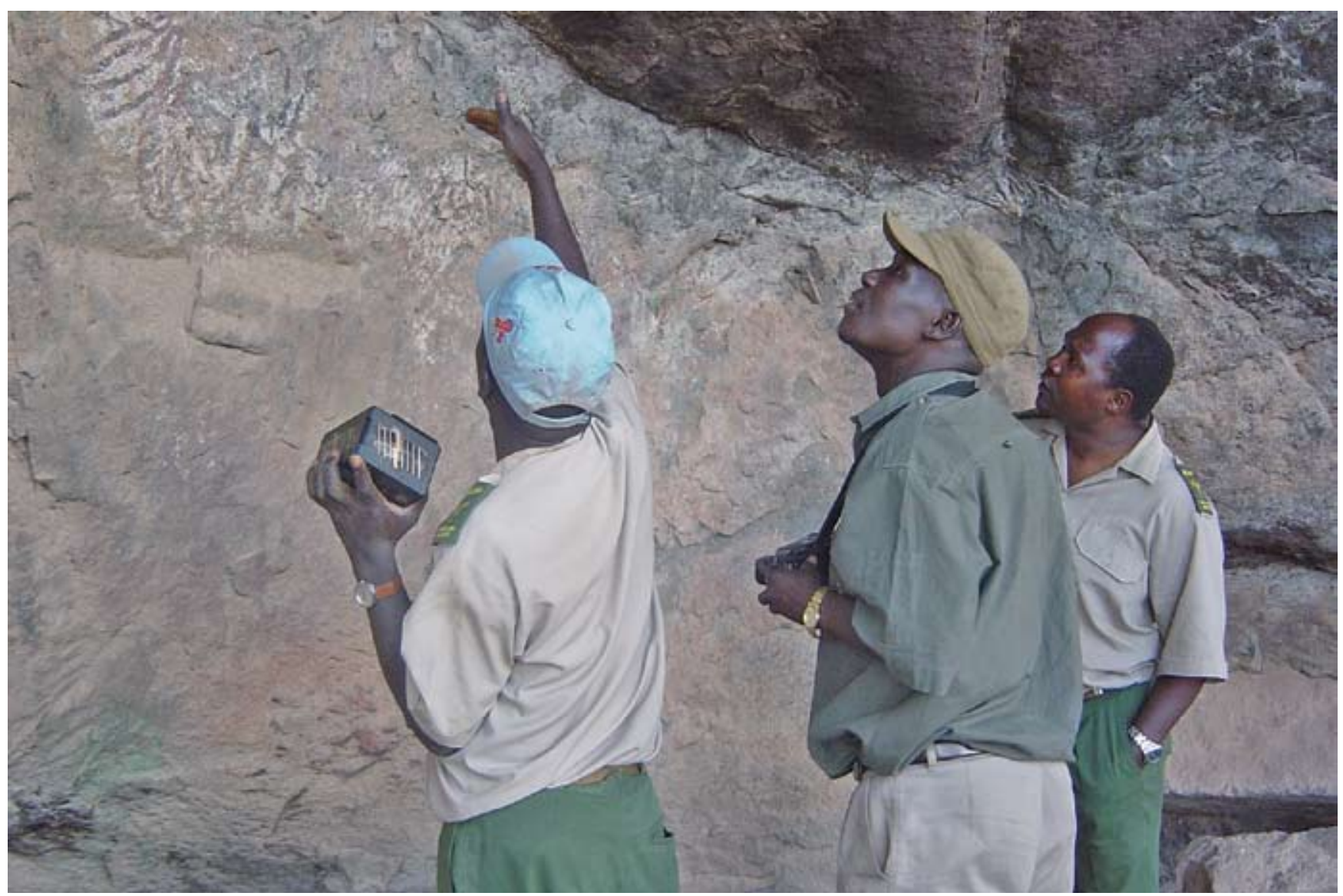

Le Parc National de Nyika (catégorie II) au Malawi contient plusieurs sites naturels sacrés et aussi d'importants vestiges de peintures rupestres ainsi qu'un taux élevé de biodiversité originelle. (C) Nigel Dudley 
- Les aires protégées pourraient devoir être déplacées (par exemple si le niveau de la mer monte) ou bien il faudra en créer de nouvelles ; dans certains cas, les aires protégées existantes pourraient n'avoir plus de raison d'être si les espèces qu'elles étaient censées protéger ne peuvent plus y survivre. Nous nous sommes habitués à considérer les aires protégées comme des entités fixées une fois pour toutes, qui restent sacro-saintes dans un avenir proche, mais dans ces nouvelles conditions de changements climatiques, il est possible que ce ne soit plus la façon la plus efficace d'en assurer la conservation.

- Il faut encourager les recherches sur les changements climatiques et les aires protégées. Elles pourront aider les gestionnaires d'aires protégées à mettre au point des réponses appropriées et pertinentes aux changements climatiques.
- Partout où c'est possible, il faut établir des aires protégées plus grandes, contenant une plus large gamme de caractéristiques biogéographiques, pour fournir de l'espace aux changements d'aire de répartition et un tampon contre les événements météorologiques extrêmes.

La plupart de ces stratégies dépassent le cadre du présent guide. Cependant, nous devons rassembler toutes les informations au sujet des mérites relatifs des différentes catégories et de la façon de les utiliser plus efficacement, en tant qu'élément clé des stratégies globales de réponse aux changements climatiques. Dans cette perspective de changements climatiques, le Tableau 5 donne une analyse préliminaire des forces, des faiblesses, des opportunités et des menaces pour chaque catégorie.

Tableau 5. Analyse forces-faiblesses-opportunités-menaces pour les catégories face aux changements climatiques

\begin{tabular}{|c|c|c|c|c|}
\hline Catégorie & Forces & Faiblesses & Opportunités & Menaces \\
\hline Catégorie la & $\begin{array}{l}\text { Une protection stricte } \\
\text { d'un environnement } \\
\text { intact offre des données } \\
\text { de base pour mesurer les } \\
\text { changements et planifier } \\
\text { les réponses. }\end{array}$ & $\begin{array}{l}\text { Souvent assez } \\
\text { petites et donc avec } \\
\text { un faible potentiel } \\
\text { d'atténuation. }\end{array}$ & $\begin{array}{l}\text { Un stress accru } \\
\text { peut nécessiter } \\
\text { plus d'intervention } \\
\text { de gestion et un } \\
\text { glissement vers, } \\
\text { p.ex., une approche } \\
\text { par catégorie IV. }\end{array}$ & \multirow[t]{2}{*}{$\begin{array}{l}\text { Laisser une } \\
\text { aire protégée } \\
\text { complètement seule } \\
\text { peut être une option } \\
\text { très risquée face } \\
\text { à un changement } \\
\text { environnemental } \\
\text { rapide. }\end{array}$} \\
\hline Catégorie lb & \multirow[t]{2}{*}{$\begin{array}{l}\text { On pense que les } \\
\text { grandes aires d'habitats } \\
\text { relativement non modifiés } \\
\text { sont les plus solides pour } \\
\text { absorber des conditions } \\
\text { climatiques changeantes } \\
\text { - avec la possibilité } \\
\text { de protéger des } \\
\text { écosystèmes entiers et } \\
\text { les processus associés. }\end{array}$} & & $\begin{array}{l}\text { Une chance de } \\
\text { préserver de très } \\
\text { grandes surfaces } \\
\text { d'habitats non } \\
\text { modifiés avec } \\
\text { un minimum } \\
\text { d'intervention } \\
\text { humaine pour } \\
\text { permettre une } \\
\text { adaptation naturelle } \\
\text { aux changements } \\
\text { climatiques. }\end{array}$ & \\
\hline Catégorie II & & & $\begin{array}{l}\text { Espace où se } \\
\text { concentrer sur les } \\
\text { approches par } \\
\text { écosystème, une } \\
\text { gestion active étant } \\
\text { déjà en place pour } \\
\text { faciliter cela. }\end{array}$ & \multirow{2}{*}{$\begin{array}{l}\text { De nombreuses } \\
\text { aires protégées } \\
\text { de catégorie II } \\
\text { et III survivent } \\
\text { grâce aux revenus } \\
\text { du tourisme, qui } \\
\text { peuvent être mis } \\
\text { en danger par } \\
\text { l'augmentation du } \\
\text { prix des produits } \\
\text { pétroliers et par les } \\
\text { campagnes contre } \\
\text { les vacances en } \\
\text { avion. }\end{array}$} \\
\hline Catégorie III & $\begin{array}{l}\text { D'habitude des sites } \\
\text { emblématiques avec } \\
\text { un engagement fort } \\
\text { envers la poursuite de la } \\
\text { conservation. }\end{array}$ & $\begin{array}{l}\text { Souvent trop petite } \\
\text { pour absorber } \\
\text { les effets des } \\
\text { changements } \\
\text { climatiques. }\end{array}$ & $\begin{array}{l}\text { Peut offrir des } \\
\text { « îlots » de protection } \\
\text { dans des paysages } \\
\text { par ailleurs fortement } \\
\text { altérés. }\end{array}$ & \\
\hline Catégorie IV & $\begin{array}{l}\text { Des interventions de } \\
\text { gestion pour préserver } \\
\text { les habitats et les } \\
\text { espèces cibles peuvent } \\
\text { être déjà inscrites dans } \\
\text { les plans du site. }\end{array}$ & $\begin{array}{l}\text { En général des } \\
\text { fragments d'habitats, } \\
\text { susceptibles } \\
\text { d'opposer une } \\
\text { faible résistance } \\
\text { aux changements } \\
\text { climatiques. }\end{array}$ & $\begin{array}{l}\text { La gestion humaine } \\
\text { est déjà en place, } \\
\text { donc ces aires } \\
\text { fournissent un } \\
\text { laboratoire utile } \\
\text { pour tester des } \\
\text { modifications de la } \\
\text { gestion. }\end{array}$ & $\begin{array}{l}\text { Perte des conditions } \\
\text { indispensables à } \\
\text { certaines espèces } \\
\text { protégées. }\end{array}$ \\
\hline
\end{tabular}


Tableau 5. Analyse forces-faiblesses-opportunités-menaces pour les catégories face aux changements climatiques (suite)

\begin{tabular}{|c|c|c|c|c|}
\hline Catégorie & Forces & Faiblesses & Opportunités & Menaces \\
\hline Catégorie V & $\begin{array}{l}\text { Stratégies de gestion à } \\
\text { long terme déjà en place. }\end{array}$ & $\begin{array}{l}\text { Une certaine } \\
\text { proportion de l'habitat } \\
\text { a déjà été altérée } \\
\text { et peut-être affaiblie } \\
\text { (p.ex. face à la } \\
\text { présence d'espèces } \\
\text { invasives). }\end{array}$ & $\begin{array}{l}\text { Coopération avec les } \\
\text { communautés locales } \\
\text { pour développer des } \\
\text { stratégies de gestion } \\
\text { adaptative dans des } \\
\text { paysages terrestres et } \\
\text { marins principalement } \\
\text { culturels. }\end{array}$ & $\begin{array}{l}\text { Abandon des terres } \\
\text { suite aux conditions } \\
\text { changeantes et } \\
\text { donc perte des } \\
\text { systèmes culturels } \\
\text { dont la biodiversité a } \\
\text { fini par dépendre. } \\
\text { Pression } \\
\text { supplémentaire sur } \\
\text { les ressources due à } \\
\text { des conditions plus } \\
\text { rudes. }\end{array}$ \\
\hline Catégorie VI & $\begin{array}{l}\text { Engagement humain } \\
\text { envers la conservation à } \\
\text { long terme. }\end{array}$ & & $\begin{array}{l}\text { Coopération avec les } \\
\text { communautés locales } \\
\text { pour développer des } \\
\text { stratégies de gestion } \\
\text { adaptative pour la } \\
\text { gestion durable. }\end{array}$ & $\begin{array}{l}\text { Un climat qui } \\
\text { change rend les } \\
\text { anciens systèmes } \\
\text { de gestion durable } \\
\text { moins viables. }\end{array}$ \\
\hline $\begin{array}{l}\text { Toutes les } \\
\text { catégories }\end{array}$ & $\begin{array}{l}\text { Préservation de systèmes } \\
\text { sains qui sont jugés être } \\
\text { les mieux adaptés pour } \\
\text { faire face aux impacts des } \\
\text { changements climatiques. } \\
\text { Préservation d'un potentiel } \\
\text { d'adaptation et d'une } \\
\text { banque génétique in-situ. }\end{array}$ & $\begin{array}{l}\text { Fixées à un endroit et } \\
\text { donc vulnérables à un } \\
\text { climat en évolution. }\end{array}$ & $\begin{array}{l}\text { Modifier les stratégies } \\
\text { de gestion en réponse } \\
\text { aux changements, } \\
\text { en s'inspirant de } \\
\text { l'expérience des } \\
\text { autres catégories et } \\
\text { de la gestion durable } \\
\text { en dehors des aires } \\
\text { protégées. }\end{array}$ & $\begin{array}{l}\text { Les changements } \\
\text { climatiques rendent } \\
\text { le site impropre pour } \\
\text { certaines espèces et } \\
\text { habitats cibles. }\end{array}$ \\
\hline
\end{tabular}

\section{Utiliser les catégories d'aires protégées de I'UICN comme un instrument de politique de la conservation}

Même s'il n'était pas prévu que les catégories deviennent des instruments de politique, en pratique elles sont souvent utilisées comme telles, par l'UICN elle-même et, plus fréquemment, par des gouvernements et par d'autres institutions. Ceux qui utilisent les catégories doivent en être bien conscients et en tenir compte dans leur application. Il existe six grands types d'utilisation politique, avec des statuts officiels divers :

- Politique descriptive internationale : là où les catégories sont adoptées officiellement pour enregistrement -c'est un des buts originaux du système de catégories. Les catégories ont été adoptées par le système des NU, par exemple dans la Liste des Nations unies des aires protégées ${ }^{8}$ et le Programme de travail sur les aires protégées de la CDB et dans la Base de données mondiale sur les aires protégées. Au niveau international, on a aussi un peu utilisé le système de catégories dans des institutions ou des accords mondiaux tels que le Forum intergouvernemental sur les forêts, l'Evaluation mondiale des ressources forestières des Nations unies, ainsi que dans le contexte des réserves de biosphère.

- Politique prescriptive internationale : de façon plus controversée, les catégories ont servi de façon limitée pour suggérer une politique internationale, y compris des contrôles sur certaines interventions de gestion au sein d'aires protégées. Ce qui fut plus significatif, c'est le développement de la position stricte de l'UICN sur l'exploration et d'exploitation minière dans les catégories I à IV. Cette recommandation $\left(\mathrm{n}^{\circ} 2.82\right)$ a été adoptée au Congrès mondial de la conservation à Amman, en Jordanie, en 2000. Elle recommande, entre autres, à tous les Etats membres de l'UICN « d'interdire par la loi toutes les activités d'exploration et d'exploitation des ressources minérales dans les aires protégées correspondant aux catégories I à $I V$ de gestion des aires protégées définies par l'UICN». Cette recommandation a joué un rôle important dans l'adoption par Shell et par le CIMM de l'engagement de ne pas pénétrer dans les sites naturels du patrimoine mondial. Ceci représente une nouvelle application du système de catégories de l'UICN dans la mesure où elle relie des restrictions de l'utilisation des ressources au système lui-même, mais elle suscite aussi d'importantes questions quant à savoir si le système est assez rigoureux pour cette utilisation.

8 Les catégories de 1994 ont été utilisées comme base pour compiler les versions de 1997 et de 2003 de la Liste des Nations unies. 
- Politique régionale : deux conventions et accords régionaux ont appliqué les catégories de l'UICN (Dillon 2004) : la Stratégie et le Plan d'action de 1996 du Réseau circumpolaire des zones protégées (CPAN) de Conservation de la faune et de la flore arctiques (CFFA) et la Convention africaine (révisée en 2003) sur la conservation de la nature et des ressources naturelles. Dans le cas de la Convention africaine, les catégories de l'UICN ont eu une forte influence sur la mise au point de la convention révisée et ont fourni un cadre pour un certain nombre de sections, adoptées d'abord par un groupe de travail inter-agences avant d'être soumises à de nombreux experts de gouvernements africains qui ont adapté le texte au contexte africain. L'article $\mathrm{V}$ de la Convention définit une Aire de conservation comme toute aire protégée désignée et gérée principalement pour une gamme d'objectifs et se poursuit en élaborant ces objectifs en référence aux six catégories de l'UICN. Un autre exemple d'application au niveau régional se trouve en Europe, où une publication de la fédération CMAP/EUROPARC fut préparée sur l'Interprétation et l'application des catégories de gestion des aires protégées en Europe, pour donner des orientations dans le contexte européen.

- Politique descriptive nationale : un certain nombre de pays ont déjà fait des efforts réfléchis pour aligner la catégorisation de leurs aires protégées sur le système de l'UICN, soit en changeant les catégories pour s'adapter directement au système, soit en s'accordant sur des équivalences pour faciliter des comparaisons croisées. Bien que l'emploi des catégories soit volontaire, la plupart des pays appliquent actuellement les catégories à certaines si pas à toutes leurs aires protégées. Quelque 10 pour cent des législations nationales en matière d'aires protégées utilisent les catégories UICN depuis 1994 ; citons entre autres les législations d'Australie, du Brésil, de Bulgarie, du Cambodge, de Cuba, de Géorgie, de Hongrie, du Koweït, du Mexique, du Niger, de Slovénie, d’Uruguay et du Viet Nam.

- Politique prescriptive nationale : un sous-ensemble plus réduit de pays ont explicitement lié leur politique aux catégories, y compris au niveau du financement (p.ex. l'Autriche) ou des politiques en matière d'implantations dans les aires protégées. Dans un certain nombre de cas, les pays ont affiné la signification des catégories dans leur contexte national ; ils conservent le cadre original mais ils ajoutent des détails dans leur politique - c'est le cas de Madagascar.

- Politique des ONG : l'utilisation par les ONG est moins officielle mais tout aussi significative. Par exemple, plusieurs ONG ne comptent, en réalité, que les catégories I-IV comme des aires protégées, ce qui influence de nombreux plans au niveau de l'écorégion ou de la biorégion. Les ONG se sont aussi servies des catégories de l'UICN pour appuyer leur cause, par exemple pour faire pression en faveur d'approches particulières de gestion dans des aires protégées.

\section{Leçons apprises de l'application du système de catégories à la politique}

A ce jour, l'expérience a révélé quelques leçons générales au sujet de l'utilisation des catégories dans la politique :

- Les catégories ont le potentiel certain d'influencer la politique et la législation en matière d'aires protégées à tous les niveaux, et le rythme de leur application s'est fortement accéléré depuis la publication des lignes directrices en 1994 ;

- L'on prévoit que l'importance relative de l'influence du système des catégories sur les décisions politiques va augmenter, particulièrement au niveau national, au fur et à mesure que le Programme de travail sur les aires protégées de la CDB sera plus largement et plus concrètement mis en œuvre ;

- L'avantage d'inclure le système de catégories dans les décisions politiques est que cela confere au système plus de poids et de crédibilité et que cela peut augmenter la sensibilisation et la compréhension des valeurs des aires protégées ;

- L'utilisation la plus efficace du système de catégories dans les décisions politiques s'observe lorsque le système est appliqué de manière flexible, en fonction des conditions nationales ou régionales particulières ;

- L'application du système de catégories assure aussi la reconnaissance en matière de normes internationales.

Il existe, cependant, un certain nombre de contraintes qui pèsent sur l'application effective du système de catégories dans les décisions politiques. Elles comprennent :

- La validité et l'exactitude du processus utilisé pour assigner les aires protégées à des catégories de l'UICN, particulièrement les catégories I à IV, ont été remises en cause : cela concernait particulièrement la recommandation de politique de "no-go " pour l'exploration minière dans les catégories I à IV de l'UICN et laissait entendre que l'utilisation en politique impliquait une plus grande rigueur dans l'application que cela n'avait été le cas par le passé ;

- Le manque de connaissance et/ou de compréhension du système de catégories de l'UICN ;

- L'exactitude variable des données sur les aires protégées intégrées dans la Base de données sur les aires protégées et dans la Liste des Nations unies des aires protégées;

- Le manque de compréhension et de sensibilisation quant à la façon d'appliquer le système de catégories au niveau national ainsi que dans certains biomes.

Il en résulte qu'à l'avenir, les efforts pour utiliser le système des catégories dans les décisions politiques devront se fonder sur une compréhension et une application plus rigoureuses des objectifs de ces catégories. 



\section{Applications spéciales}

Les aires protégées comprennent une gamme énorme de biomes, de formes de propriété et de motivations - qui toutes ont un impact sur la façon dont les objectifs de gestion sont fixés et donc sur les catégories qui sont ensuite appliquées. Cette section examine en détails certains cas particuliers qui ont amené de la confusion dans le passé : les aires protégées forestières, d'eau douce ou marines, les sites naturels sacrés et le rôle de la restauration dans la protection. 


\section{Aires protégées forestières}

Il y a eu une certaine confusion au sujet d'aires protégées forestières, et particulièrement à propos de ce qui " compte " comme aire protégée dans le biome forestier, spécialement lorsque cette information est intégrée dans une vaste récolte de données concernant les ressources forestières. Les lignes directrices suivantes (basées sur Dudley et Phillips 2006) abordent une série de questions, y compris :

- La définition d'une forêt dans le contexte d'aires protégées forestières ;

- L'application du système des catégories UICN à des forêts ;

- Le calcul de l'étendue des aires protégées forestières ;

- Les zones qui ne rentrent pas dans la définition UICN d'une aire protégée forestière ;

- La distinction entre corridors biologiques, étapes relais et zones tampons intérieures.

\section{Définition d'une forêt dans le contexte des aires protégées}

La définition s'inspire de celle de l'UNECE/FAO et y ajoute l'interprétation de l'UICN comme suit :

\section{Définition de la forêt par l'UNECE/FAO}

Forêt : Terre portant une couverture forestière (ou la densité de peuplement équivalente) supérieure à 10 pour cent et d'une superficie de plus de 0,5 ha. Les arbres doivent pouvoir atteindre plus de $5 \mathrm{~m}$ de haut à maturité in situ. Une forêt peut consister en une formation forestière dense où les arbres de différentes hauteurs et le sous-bois couvrent une portion importante du sol ainsi que les formations forestières claires avec une strate végétale continue où le couvert arboré est supérieur à 10 pour cent. Les jeunes peuplements naturels et toutes les plantations d'exploitation qui n'ont pas encore atteint une densité de 10 pour cent au niveau de la couronne ou une hauteur de $5 \mathrm{~m}$ sont inclus dans le terme de forêt, tout comme les aires qui font normalement partie de la superficie forestière mais qui sont temporairement déboisées du fait d'une intervention humaine ou de causes naturelles mais qui doivent redevenir boisées.

Cela comprend : Les pépinières ou les vergers semenciers qui constituent une partie intégrante d'une forêt ; les routes forestières, les pistes dégagées, les coupe-feux et autres petites surfaces dégagées, des forêts dans les parcs nationaux, les réserves naturelles et autres aires protégées, comme celles d'intérêt scientifique, historique, culturel ou spirituel spécifique ; les coupe-vent et les ceintures refuges arborées de plus de 0,5 ha et d'une largeur de plus de $20 \mathrm{~m}$; des plantations utilisées d'abord à des fins de foresterie, y compris les plantations d'hévéas et les bosquets de chênes-lièges.
Cela ne comprend pas : Les terres utilisées principalement pour des pratiques agricoles.

Autres terres boisées : Les terres portant une couverture forestière (ou la densité de peuplement équivalente) de 5 à 10 pour cent d'arbres qui doivent pouvoir atteindre une hauteur de $5 \mathrm{~m}$ à maturité in situ; ou une couverture forestière (ou la densité de peuplement équivalente) de plus de 10 pour cent d'arbres qui n'atteignent pas une hauteur de $5 \mathrm{~m}$ à maturité in situ (p.ex. des arbres nains ou rabougris); ou avec une couverture de broussailles ou de buissons de plus de 10 pour cent.

Note d'orientation politique : Pour des forêts qui se trouvent dans des aires protégées forestières, la définition de l'UNECE/ FAO doit être utilisée avec les caveat suivants :

- Les plantations forestières dont l'objectif de gestion principal est le bois rond industriel, la gomme, la résine ou les fruits ne doivent pas être comptées ;

- Les terres restaurées en forêts naturelles doivent être comptées si le principal objectif de gestion est le maintien et la protection de la biodiversité et des valeurs culturelles associées ;

- Les «forêts culturelles " doivent être inclues si elles sont protégées en premier lieu pour leur biodiversité et pour les valeurs culturelles associées.

\section{Appliquer le système des catégories de I'UICN aux forêts}

Une grande partie de la confusion possible au sujet de ce qui est ou n'est pas une aire protégée peut être évitée si l'on souligne la nature hiérarchisée de la définition et que le système est appliqué par étapes. En bref, les catégories ne doivent s'appliquer aux aires protégées forestières que si les aires en question répondent déjà à la définition d'une aire protégée. Même après qu'une aire protégée a été correctement identifiée, il encore est possible de se tromper quand on décide à quelle catégorie l'assigner. Deux questions se posent :

- Quelle portion d'une aire protégée doit être boisée avant de la compter comme aire protégée forestière ? Certaines forêts importantes situées à l'intérieur d'aires protégées peuvent en fait être un habitat minoritaire, comme une forêt relictuelle, une forêt riveraine ou des mangroves. Cela crée des problèmes d'interprétation et de disponibilité de données. Les statisticiens forestiers doivent-ils différencier les fractions d'aires protégées qui contiennent de la forêt?

- Toute la forêt située dans une aire protégée est-elle automatiquement une aire protégée forestière ? Certaines aires protégées, spécialement des catégories $\mathrm{V}$ et VI peuvent contenir des surfaces boisées qui ne sont pas des forêts protégées, telles que les plantations d'espèces exotiques dans de nombreuses aires protégées de catégorie $\mathrm{V}$ en Europe. 
Celles-ci ne répondent pas à la définition d'une forêt que l'on propose d'utiliser dans les aires protégées et qui est expliquée plus haut, mais elles sont parfois enregistrées comme " protégées » - et peuvent donc figurer dans les statistiques officielles comme " aires protégées forestières".

Il est important de suivre une procédure normalisée pour déterminer l'étendue d'aires protégées forestières et obtenir des données significatives et exactes. Le calcul doit suivre la séquence expliquée ci-dessous. Les aires protégées forestières peuvent être calculées comme un sous-ensemble sans équivoque des statistiques nationales sur les aires protégées, en récoltant les informations sur toutes les forêts protégées mais éliminant les plantations présentes à l'intérieur des catégories moins strictement protégées.

Orientation politique et interprétation : Le processus d'assignation doit donc commencer par la définition de l'UICN d'une aire protégée et ensuite être affiné en référence aux catégories de l'UICN :

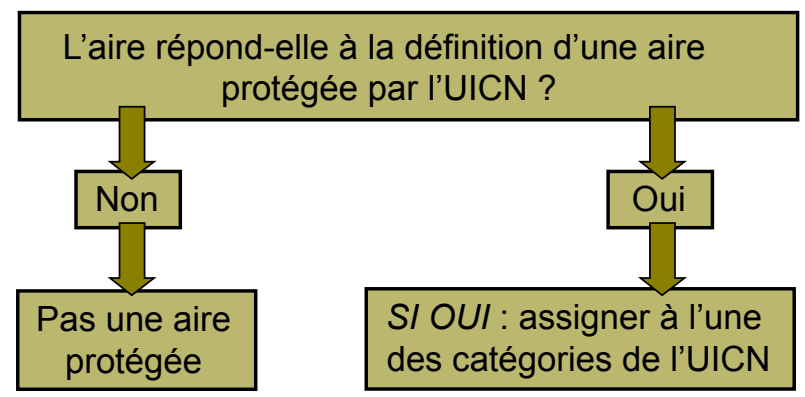

Il en résulte que toute aire qui semble correspondre à une des catégories sur la seule base de ses pratiques de gestion, mais qui ne répond pas à la définition générale d'une aire protégée, ne doit pas être considérée comme une aire protégée selon la définition de l'UICN.

\section{Calcul de l'étendue des aires protégées forestières}

Lorsque l'on a besoin de statistiques spécifiques sur les forêts, il est nécessaire d'identifier la proportion d'aires protégées qui contient de la forêt. Ceci est rarement simple à faire : de nombreuses aires protégées contiennent de la forêt ; même des " aires protégées forestières " ne sont souvent pas entièrement boisées; et, de plus, le calcul doit parfois prendre en compte des forêts qui sont soumises à un régime de protection élargi à l'échelle du paysage et qui ne répondent pas aux critères d'identification indiqués plus haut.

Orientation politique : Le calcul des aires protégées forestières passe par les étapes suivantes :

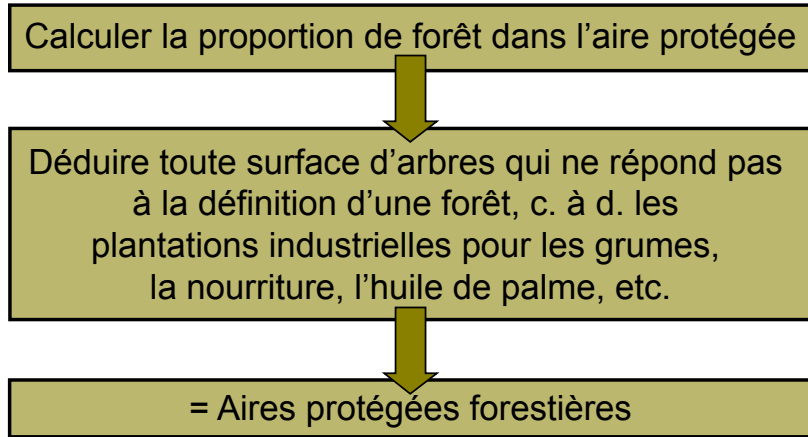

\section{Quelles aires n'entrent pas dans la définition de I'UICN d'une aire protégée forestière ?}

De nombreuses utilisations forestières - dont certaines ont une grande valeur sociale, écologique ou biologique - n'entrent pas dans la définition de l'UICN.

Orientation politique : les forêts suivantes ne sont pas automatiquement des aires protégées forestières :

- Les forêts gérées pour la protection de ressources autres que la biodiversité - p.ex. des forêts mises en réserve pour la protection d'un bassin versant ou de l'eau potable, pour le contrôle des avalanches, les coupe-feux, les coupe-vent et le contrôle de l'érosion ;

- Les forêts gérées d'abord comme une ressource communautaire - p.ex. les forêts gérées pour les produits forestiers non ligneux, le bois de feu et le fourrage, à des fins récréatives ou religieuses ;

- Les forêts gérées comme une ressource stratégique - p.ex. comme une source de bois d'urgence, en temps de conflits ;

- Les forêts dont les principaux objectifs de gestion ne sont pas clairs, avec pour conséquence que la protection de la biodiversité y est considérée comme un objectif égal, voire moins prioritaire que d'autres utilisations ;

- Les forêts mises en réserve par hasard - p.ex. les forêts situées dans la bande médiane ou en bordure d'autoroutes, ou des forêts conservées pour des raisons militaires ou sécuritaires.

Le Tableau 6 en donne quelques exemples. 
Tableau 6. Exemples d'aires protégées forestières, ainsi que de forêts bien conservées qui ne sont pas des aires protégées forestières

\begin{tabular}{|c|c|c|}
\hline Type de forêt & Exemple & Notes \\
\hline \multicolumn{3}{|c|}{ Exemples d'aires protégées forestières } \\
\hline $\begin{array}{l}\text { Aire protégée de } \\
\text { catégorie la de } \\
\text { I'UICN }\end{array}$ & $\begin{array}{l}\text { Wolong Nature } \\
\text { Reserve, Sichuan, } \\
\text { Chine }\end{array}$ & $\begin{array}{l}\text { Une aire protégée stricte, établie prioritairement pour protéger le panda } \\
\text { géant, y compris un centre de reproduction en captivité. }\end{array}$ \\
\hline $\begin{array}{l}\text { Aire protégée de } \\
\text { catégorie II de } \\
\text { I'UICN }\end{array}$ & $\begin{array}{l}\text { Parc National de } \\
\text { Huerquehue, Chili }\end{array}$ & $\begin{array}{l}\text { Ce parc national est entièrement protégé (il s'y trouve certaines } \\
\text { propriétés privées à l'intérieur mais elles sont exclues de l'aire protégée } \\
\text { et elles servent pour l'écotourisme). Il fut créé principalement pour la } \\
\text { préservation des forêts d'Araucaria (désespoir des singes) uniques. }\end{array}$ \\
\hline $\begin{array}{l}\text { Catégorie III de } \\
\text { I'UICN }\end{array}$ & $\begin{array}{l}\text { Aire à usages } \\
\text { multiples de } \\
\text { Monterrico, } \\
\text { Guatemala }\end{array}$ & $\begin{array}{l}\text { C'est une aire côtière contenant le plus grand bloc de mangroves } \\
\text { qui reste dans le pays, des plages à tortues ainsi que plusieurs } \\
\text { communautés marines. Les mangroves sont gérées pour la protection } \\
\text { et la pêche artisanale. }\end{array}$ \\
\hline $\begin{array}{l}\text { Catégorie IV de } \\
\text { I'UICN }\end{array}$ & $\begin{array}{l}\text { Réserve de Faune } \\
\text { du Dja, Cameroun }\end{array}$ & $\begin{array}{l}\text { Dans le bassin du fleuve Congo, au sud-est du Cameroun. De } \\
\text { nombreuses personnes vivent dans et autour de l'aire protégée, y } \\
\text { compris des tribus du peuple (pygmée) baka. La gestion doit être } \\
\text { active pour contrôler le commerce de viande de brousse et pour aider à } \\
\text { restaurer les surfaces de forêt. }\end{array}$ \\
\hline $\begin{array}{l}\text { Catégorie V de } \\
\text { I'UICN }\end{array}$ & $\begin{array}{l}\text { Sugarloaf Mountain, } \\
\text { Brecon Beacons } \\
\text { National Park, UK }\end{array}$ & $\begin{array}{l}\text { Les bois sur les flancs de la montagne sont la propriété du National } \\
\text { Trust, une grande ONG, et gérés par lui comme une aire protégée } \\
\text { même si un broutage limité par les moutons est autorisé dans l'aire } \\
\text { protégée forestière. Les collines environnantes servent de pâturages } \\
\text { aux moutons. }\end{array}$ \\
\hline $\begin{array}{l}\text { Catégorie VI de } \\
\text { I'UICN }\end{array}$ & $\begin{array}{l}\text { Réserve } \\
\text { Anthropologique de } \\
\text { Talamanca Cabécar, } \\
\text { Costa Rica }\end{array}$ & $\begin{array}{l}\text { Une certaine exploitation forestière est autorisée dans cette aire } \\
\text { protégée, spécialement aux populations autochtones, mais la plus } \\
\text { grande partie reste sous une protection stricte. }\end{array}$ \\
\hline \multicolumn{3}{|c|}{ Exemples de forêts qui ne sont pas des aires protégées forestières } \\
\hline $\begin{array}{l}\text { Forêt dans la } \\
\text { catégorie V de } \\
\text { l'UICN }\end{array}$ & $\begin{array}{l}\text { Plantation forestière } \\
\text { dans le Snowdonia } \\
\text { National Park, pays } \\
\text { de Galles, UK }\end{array}$ & $\begin{array}{l}\text { Bien que la plantation se trouve dans une aire protégée de catégorie } \\
V \text {, c'est une plantation d'état, entièrement commerciale, d'espèces } \\
\text { exotiques qui, en tant que telle, ne constitue pas une aire protégée } \\
\text { forestière. }\end{array}$ \\
\hline $\begin{array}{l}\text { Forêt gérée } \\
\text { pour le contrôle } \\
\text { environnemental }\end{array}$ & $\begin{array}{l}\text { Brisbane watershed, } \\
\text { Queensland, } \\
\text { Australie }\end{array}$ & $\begin{array}{l}\text { Certaines parties de la zone de captage autour de Brisbane sont mises } \\
\text { en réserve pour éviter la coupe de grumes et d'autres perturbations } \\
\text { afin de préserver l'approvisionnement en eau de la ville. La forêt est } \\
\text { strictement préservée mais pas comme une aire protégée puisqu'il n'y a } \\
\text { aucun objectif spécial concernant la protection de la biodiversité, alors } \\
\text { qu'il y a aussi des aires protégées qui constituent une composante de } \\
\text { l'aire de captage. }\end{array}$ \\
\hline $\begin{array}{l}\text { Forêt gérée par } \\
\text { la communauté }\end{array}$ & $\begin{array}{l}\text { La communauté } \\
\text { locale de Kribi, dans } \\
\text { le sud-ouest du } \\
\text { Cameroun }\end{array}$ & $\begin{array}{l}\text { La population locale gère une forêt dans le cadre d'un projet facilité par } \\
\text { le WWF. La forêt doit apporter des bénéfices tant à la population locale } \\
\text { qu'à l'environnement mais elle n'est pas désignée comme aire protégée } \\
\text { (et n'a pas d'objectif particulier visant la protection de la biodiversité). }\end{array}$ \\
\hline $\begin{array}{l}\text { Forêt gérée } \\
\text { à des fins } \\
\text { multiples }\end{array}$ & $\begin{array}{l}\text { Les forêts du massif } \\
\text { du Jura, Suisse }\end{array}$ & $\begin{array}{l}\text { La politique forestière suisse met l'accent sur la gestion à des fins } \\
\text { multiples, l'abattage sélectif d'arbres et la conservation. Le Jura est } \\
\text { une ressource importante pour la communauté locale et pour la faune. } \\
\text { Cependant, la région dans son ensemble n'est pas une aire protégée } \\
\text { même si certaines aires protégées (de diverses catégories) se trouvent } \\
\text { à l'intérieur. }\end{array}$ \\
\hline $\begin{array}{l}\text { Forêts } \\
\text { protégées par } \\
\text { hasard }\end{array}$ & $\begin{array}{l}\text { Forêts sur la frontière } \\
\text { entre Corée du Nord } \\
\text { et du Sud (la zone } \\
\text { démilitarisée) }\end{array}$ & $\begin{array}{l}\text { De grandes surfaces de forêts sont complètement préservées du fait } \\
\text { de l'exclusion de toute activité de défense, mais cette situation pourrait } \\
\text { être modifiée si la situation politique change. }\end{array}$ \\
\hline
\end{tabular}




\section{Distinguer les corridors biologiques, les étapes relais et les zones tampons à l'intérieur et à l'extérieur des aires protégées forestières}

L'UICN propose aussi des lignes directrices pour identifier quand certains habitats de jonction importants - comme les corridors et les zones tampons - rentrent ou pas dans la définition d'une aire protégée (voir Tableau 7 ci-dessous).

Tableau 7. Distinguer les aires de conservation de la connectivité telles que les corridors, les étapes-relais biologiques et les zones tampons à l'intérieur et à l'extérieur des aires protégées

\begin{tabular}{|c|c|c|}
\hline Elément & Description & Exemples \\
\hline $\begin{array}{l}\text { Corridor } \\
\text { biologique }\end{array}$ & $\begin{array}{l}\text { Aire d'habitat approprié, ou d'habitat } \\
\text { en voie de restauration, reliant deux } \\
\text { ou plusieurs aires protégées (ou des } \\
\text { habitats importants non protégés) } \\
\text { pour permettre les mouvements et } \\
\text { migrations d'espèces, les échanges } \\
\text { génétiques, etc. }\end{array}$ & $\begin{array}{l}\text { Aires protégées } \\
\text { - Désignation d'une forêt qui relie deux forêts protégées } \\
\text { existantes pour constituer une aire totalement protégée } \\
\text { dans une catégorie UICN } \\
\text { Aires non protégées } \\
\text { - Aires de forêt certifiée pour bonne gestion entre des } \\
\text { aires protégées forestières } \\
\text { - Aire boisée reliant deux aires protégées et } \\
\text { volontairement gérée pour la vie sauvage par son } \\
\text { propriétaire, sur une base temporaire } \\
\text { - Aire forestière couverte par une servitude de } \\
\text { conservation détenue par le gouvernement ou par une } \\
\text { organisation de conservation privée }\end{array}$ \\
\hline $\begin{array}{l}\text { Etape-relais } \\
\text { écologique }\end{array}$ & $\begin{array}{l}\text { Aire d'habitat approprié ou d'habitat } \\
\text { en voie de restauration situé entre } \\
\text { deux aires protégées ou d'autres } \\
\text { types d'habitats importants qui } \\
\text { fournissent un habitat temporaire } \\
\text { pour des oiseaux migrateurs et } \\
\text { d'autres espèces. }\end{array}$ & $\begin{array}{l}\text { Aires protégées } \\
\text { - Forêts relictuelles gérées pour offrir des points de } \\
\text { chute aux oiseaux migrateurs } \\
\text { Aires non protégées } \\
\text { - Terres boisées mises en jachère par des fermiers suite } \\
\text { à des accords volontaires, et avec des compensations } \\
\text { du gouvernement, pour offrir un habitat temporaire à } \\
\text { des oiseaux migrateurs }\end{array}$ \\
\hline Zone tampon & $\begin{array}{l}\text { Aire entourant une zone noyau } \\
\text { protégée, qui est gérée pour aider } \\
\text { à préserver les valeurs de l'aire } \\
\text { protégée. }\end{array}$ & $\begin{array}{l}\text { Aires protégées } \\
\text { - Forêt à la limite d'une aire protégée, qui est ouverte } \\
\text { à l'utilisation par la communauté, avec des contrôles } \\
\text { écologiques, et sans impact sur l'objectif de } \\
\text { conservation. Généralement une aire protégée de } \\
\text { catégorie V ou VI entourant un cœur plus strictement } \\
\text { protégé (I à IV). Dans certains pays, les zones } \\
\text { tampons sont légalement déclarées comme faisant } \\
\text { partie de l'aire protégée. } \\
\text { Aires non protégées } \\
\text { - Forêts se trouvant en dehors d'une aire protégée et } \\
\text { qui sont gérées avec précaution par des accords avec } \\
\text { les communautés locales, avec ou sans compensation } \\
\text { financière. }\end{array}$ \\
\hline
\end{tabular}

\section{Aires marines protégées}

Les aires marines protégées (AMP), de par leur nature, présentent en matière de gestion une série de défis qui peuvent nécessiter des approches différentes de celles d'aires protégées dans des environnements terrestres. Certaines caractéristiques particulières des aires protégées du milieu marin, souvent absentes ou rares sur terre, sont que :

- Les AMP sont désignées dans un environnement tridimensionnel ; on peut envisager différentes approches de gestion en fonction de la profondeur (voir la discussion au point 3 ci-dessous) ; 
- Il existe en général des flux multidirectionnels (p.ex. des marées, des courants) ;

- Le droit foncier s'applique rarement dans l'environnement marin ; le plus souvent, les aires marines sont considérées comme étant des biens communs où tous les utilisateurs ont un droit d'accès et d'usage ;

- La protection intégrale peut n’être nécessaire qu’à certaines périodes de l'année, par exemple pour protéger des sites de reproduction de poissons ou de mammiferes marins ;

- Le contrôle de l'entrée et des activités dans des aires marines protégées est souvent particulièrement difficile (et souvent impossible) à réglementer et à exercer, et les limites ou les restrictions face à des influences externes peuvent rarement être appliquées ;

- Les aires marines protégées sont sujettes aux influences environnantes et particulièrement aux effets " rabattants " (des effluents) qui se produisent souvent hors de la sphère de contrôle de la gestion, et il est même plus difficile de gérer des aires marines protégées comme unités séparées que ça l'est sur la terre ferme ;

- L'échelle sur laquelle se produit la connectivité en milieu marin peut être très grande.

Aujourd'hui, il existe quelque 5000 AMP et nombre d'entre elles ont été assignées à une ou plusieurs catégories de l'UICN. Cependant, l'application des catégories dans l'environnement marin est actuellement souvent inexacte. De plus, dans les cas où les aires protégées couvrent des surfaces de terre et de mer, les objectifs marins sont souvent ignorés lorsque l'on assigne une catégorie au site. De telles incohérences entre des AMP similaires réduisent l'efficacité et la pertinence du système en tant que programme de classification global. Cette section des lignes directrices a pour but d'aider à accroître l'exactitude de l'assignation et de la description.

\section{Principes généraux pour appliquer les catégories aux AMP (ou à une zone incluse dans une AMP)}

1. Distinguer les AMP des autres aires gérées pour l'une ou l'autre forme de conservation

Pour qu'une aire soit considérée comme une aire marine protégée, elle doit répondre à la définition générale d'une aire protégée par l'UICN : certains sites mis en réserve d'abord pour d'autres raisons (p.ex. pour des raisons militaires) peuvent avoir une grande valeur pour la biodiversité marine mais ne pas être classés comme aires marines protégées.

La définition d'une AMP utilisée par l'UICN depuis 1999 est : "Tout espace intertidal ou infratidal ainsi que ses eaux sous-jacentes, sa flore, sa faune et ses ressources historiques et culturelles que la loi ou d'autres moyens efficaces ont mis en réserve pour protéger en tout ou en partie le milieu ainsi délimité" (Kelleher 1999).
La nouvelle définition générale d'une aire protégée par l'UICN (voir page 10) remplace la définition d'une AMP de 1999 dans les aires marines. Bien qu'elle ait perdu sa référence spécifique à l'environnement marin, elle garantit une démarcation plus claire entre les sites orientés vers la conservation et ceux dont la raison d'être première est une utilisation extractive, c.-à-d. les zones de gestion de la pêche. Elle n'empêche pas l'inclusion des zones adéquates de protection de la pêche, mais celles-ci doivent respecter la nouvelle définition pour être acceptées comme AMP par la CMAP-Marine. Donc, toutes les aires de la mer qui sont consacrées d'une manière ou d'une autre à la conservation seront qualifiées, et pour celles qui ne le sont pas, la façon d'évoluer vers la reconnaissance officielle de l'UICN comme AMP est claire.

Comme avec les aires protégées terrestres, il existe une large gamme de types de gouvernance. Par exemple, de nombreuses petites AMP gérées par les communautés ont été établies particulièrement dans le Pacifique et le Sud-est asiatique. Actuellement, elles ne sont pas toutes reconnues comme AMP par les agences nationales et peuvent donc ne pas figurer sur les listes nationales ou internationales, ni avoir été assignées à une catégorie. Un exemple est le Samoa occidental, où un réseau de plus de 50 petites réserves de poisson villageoises a été établi dans le cadre du Plan de gestion des pêcheries villageoises (Sulu et al. 2002). Les catégories de l'UICN sont censées s'appliquer à toute forme d'approche de gestion efficace, juridique ou autre, et les aires marines protégées communautaires peuvent être reconnues comme des aires protégées et classées selon leurs objectifs de gestion pour autant qu'elles respectent la définition d'une aire protégée.

\section{Protection temporaire}

Certains sites, comme les frayères ou les voies de migration pélagiques, sont extrêmement importants, et les espèces concernées sont très vulnérables à certaines périodes spécifiques et bien connues de l'année, mais le reste du temps, ces sites ne requièrent aucune gestion plus contrôlée que les zones environnantes. L'Irish Sea Cod Box, par exemple, a été conçu comme zone spéciale pour préserver les stocks de cabillaud dans la mer d'Irlande en réduisant les activités de pêche pendant la période de frai. L'UE a encouragé l'établissement de tels " boxes" de conservation dans lesquels on exerce des contrôles saisonniers ou à temps plein, temporaires ou permanents des méthodes de pêche et/ou de l'accès. Ces sites pourraient se qualifier comme AMP s'ils respectent la définition d'une aire protégée.

\section{Application des catégories dans des AMP zonées verticalement}

Dans un environnement marin tridimensionnel, quelques juridictions ont introduit un zonage vertical (p.ex. des règles différentes dans la colonne d'eau de celles en vigueur au fond de la mer) qui fait que différentes catégories de l'UICN correspondent à différentes profondeurs de la colonne d'eau. 
Ceci peut être un moyen de tenter d'augmenter la protection benthique tout en permettant la pêche pélagique, mais ceci engendre un défi quant il s'agit de mettre ces mesures en vigueur, et le zonage vertical n'est pas facile à représenter dans les bases de données bidimensionnelles existantes ou sur des cartes. Mais ce qui est plus important, c'est que les liens entre systèmes et espèces benthiques et pélagiques peuvent ne pas être bien connus, de sorte que l'exploitation de la pêche en surface ou à moyenne profondeur peut avoir des impacts écologiques inconnus sur les communautés benthiques sous-jacentes. Pour ces raisons, la CMAP-Marine déconseille le zonage tridimensionnel. Pour la poignée d'aires marines protégées où cette situation existe, l'UICN conseille actuellement de catégoriser les aires marines protégées selon les régimes de gestion les moins restrictifs. Par exemple, si le système benthique est strictement protégé et que la zone pélagique est ouverte à une utilisation gérée des ressources compatible avec la catégorie VI, toute l'aire doit se voir assigner la catégorie VI. Ceci minimise le plus haut niveau de protection accordé (et obscurcit l'objectif original de protection benthique). Cependant, seuls une poignée de sites sont concernés par une telle situation, et l'utilisation de la catégorie la moins restrictive reflète probablement l'incertitude écologique quant à savoir si des niveaux de protection benthique plus élevés sont efficaces dans de telles circonstances.

\section{L'utilisation du zonage dans les aires marines protégées à usages multiples}

Généralement, les aires marines protégées comprennent des écosystèmes marins fluides et dynamiques, une grande diversité d'habitats et d'espèces au sein d'une même aire, et des espèces marines très migratrices. Cette complexité dicte souvent la nécessité de multiples objectifs et de programmes de gestion complexes. Dans l'environnement marin, ceci est particulièrement important, et le zonage est conseillé dans les lignes directrices des bonnes pratiques de l'UICN pour les AMP comme la meilleure façon de gérer des aires marines à usage multiple (Kelleher 1999; Day 2002).

Les AMP à usage multiple contiennent tout un spectre de zones, chaque type de zone ayant différents objectifs : certains autorisent une plus grande utilisation et un prélèvement des ressources plus important que d'autres (p.ex. des zones de nonprélèvement sont fréquemment désignées comme une des zones d'une AMP à usage multiple).

La CMAP reconnaît le problème que pose le traitement des zones dans le système de catégories. Comme dans les aires protégées terrestres, des unités de gestion individuelles dans des AMP peuvent faire l'objet d'une responsabilité et de rapports séparés, si :
- Les aires concernées ont été définies dans la législation principale ou dans un plan de gestion légalisé ;

- Ces aires sont clairement définies et cartographiées ;

- Les buts de la gestion pour les zones individuelles ne sont pas ambigus et permettent dès lors leur assignation à une catégorie donnée d'aire protégée.

L'on propose que cette approche ne soit utilisée que pour de vastes AMP à usage multiple où les zones sont clairement définies et constituent plus de 25 pour cent de la superficie totale (voir page 43 pour une explication de la " règle des 75 pour cent ").

L'identification de zones dans les AMP devrait se fonder sur les connaissances scientifiques et les expériences les meilleures et elle devrait aussi être faite après consultation des parties prenantes adéquates.

Par exemple, l'entrée amendée de la Grande Barrière de Corail dans la Liste des $N U$ des aires protégées produite par le WCMC/PNUE se présente comme le montre le Tableau 8 ci-dessous :

Tableau 8. Catégorisation de la Grande Barrière de Corail

\begin{tabular}{|l|l|c|}
\hline Aire & $\begin{array}{l}\text { Catégorie } \\
\text { UICN }\end{array}$ & Taille (ha) \\
\hline $\begin{array}{l}\text { Great Barrier } \\
\text { Reef Marine Park } \\
\text { comprenant : }\end{array}$ & 34.440 .000 \\
\hline $\begin{array}{l}\text { Grande Barrière de } \\
\text { Corail }\end{array}$ & la & 86.500 \\
\hline $\begin{array}{l}\text { Grande Barrière de } \\
\text { Corail }\end{array}$ & II & 11.453 .000 \\
\hline $\begin{array}{l}\text { Grande Barrière de } \\
\text { Corail }\end{array}$ & IV & 1.504 .000 \\
\hline $\begin{array}{l}\text { Grande Barrière de } \\
\text { Corail }\end{array}$ & VI & 21.378 .000 \\
\hline $\begin{array}{l}\text { Commonwealth } \\
\text { Islands }\end{array}$ & & 18.500 \\
\hline
\end{tabular}

\section{Appliquer les différentes catégories dans les AMP}

N'importe quelle catégorie peut être appliquée dans des environnements marins, même si certaines sont plus appropriées que d'autres. Le Tableau 9, tout en ne faisant pas autorité, donne certaines indications quant à la gamme d'approches de gestion et où elles peuvent s'appliquer. Cette orientation supplémentaire doit se lire en conjonction avec les descriptions plus détaillées de chaque catégorie que donnent ces lignes directrices.

9 Noter que les Commonwealth Islands font juridiquement partie du GBR Marine Park alors que la plupart des autres îles, qui sont sous juridiction de l'Etat, n'en font pas partie. 
Tableau 9. Application des catégories dans les aires marines protégées

\begin{tabular}{|c|c|}
\hline Catégorie & Notes relatives à l'utilisation dans les AMP \\
\hline la & $\begin{array}{l}\text { Dans ces AMP, l'objectif est la préservation de la biodiversité et des autres valeurs dans une aire } \\
\text { strictement protégée. Les zones de non-prélèvement /réserves marines sont le type spécifique } \\
\text { d'AMP qui atteignent ce résultat. Elles sont devenues un outil important pour la protection de } \\
\text { la biodiversité marine et pour la gestion de la pêche (Palumbi 2001, Roberts et Hawkins 2000). } \\
\text { Elles peuvent comprendre une AMP complète ou, souvent, être une zone séparée au sein d'une } \\
\text { AMP à usage multiple. Tout prélèvement d'espèce marine ou toute modification, extraction ou } \\
\text { récolte de ressources marines (p.ex. par la pêche, la récolte, le dragage, l'exploitation minière } \\
\text { ou le forage) est incompatible avec cette catégorie, à quelques exceptions près comme la } \\
\text { recherche scientifique. Les visites sont limitées pour garantir la préservation des valeurs de } \\
\text { conservation. Mettre en réserve des aires strictement protégées dans un environnement marin } \\
\text { est d'une importance fondamentale, spécialement pour protéger les zones de reproduction } \\
\text { et de frai des poissons et pour offrir des zones scientifiques de référence aussi intactes que } \\
\text { possible. Mais ces aires sont extrêmement difficiles à délimiter (les bouées peuvent servir de } \\
\text { points d'agrégation des poissons, ce qui annule le caractère intact de l'aire) et donc à faire } \\
\text { respecter. Chaque fois que l'on envisage des aires pour la catégorie la, les utilisations des } \\
\text { eaux environnantes, et spécialement les influences des courants et certains aspects de la } \\
\text { connectivité, doivent faire partie de l'évaluation des critères. Les aires de catégorie la doivent } \\
\text { être habituellement considérées comme des zones « noyaux » entourées d'autres aires } \\
\text { protégées gérées de manière adéquate (c.-à-d. que l'aire qui entoure l'aire de catégorie la doit } \\
\text { aussi être protégée de telle façon qu'elle complète et assure la protection de la biodiversité de la } \\
\text { zone noyau de catégorie la). }\end{array}$ \\
\hline $\mathrm{lb}$ & $\begin{array}{l}\text { Les aires de catégorie lb dans l'environnement marin doivent être des sites de paysage } \\
\text { marin relativement intact, significativement dépourvus de perturbation humaine, de travaux } \\
\text { ou d'installations et capables de rester tels grâce à une gestion efficace. La question de «" } \\
\text { nature sauvage » dans l'environnement marin est moins claire que dans les aires protégées } \\
\text { terrestres. Pour autant que ces aires soient relativement tranquilles et dépourvues d'influences } \\
\text { humaines, des qualités comme la « solitude », le « calme » ou la " jouissance d'aires naturelles } \\
\text { qui conservent leur caractère sauvage " peuvent s'apprécier facilement en plongeant sous la } \\
\text { surface. Le problème des accès par engins motorisés n'est pas un facteur aussi critique que } \\
\text { dans les aires de nature sauvage étant donné l'espace immense des océans et le fait que de } \\
\text { nombreuses aires de cette sorte ne seraient pas accessibles autrement. Il est plus important, par } \\
\text { contre, de minimiser la densité d'utilisation pour garantir que la « sensation de nature sauvage » } \\
\text { est préservée dans des aires qui sont considérées comme appropriées pour la catégorie lb. Par } \\
\text { exemple, des points d'ancrage fixes peuvent être un moyen de gérer la densité et de limiter les } \\
\text { impacts sur le fond marin tout en permettant un accès. }\end{array}$ \\
\hline II & $\begin{array}{l}\text { Les aires de catégorie II représentent un défi particulier pour l'environnement marin parce } \\
\text { qu'elles sont gérées pour la « protection de l'écosystème ॥, avec des dispositions pour les } \\
\text { visites, les activités récréatives et le tourisme nature. Dans des environnements marins, } \\
\text { l'utilisation extractive (de matériel mort ou vivant) comme activité clé est généralement } \\
\text { incompatible avec les objectifs des aires de la catégorie II. Ceci est dû au fait que de } \\
\text { nombreuses activités humaines, même pratiquées à faible échelle (comme la pêche) sont } \\
\text { maintenant considérées comme exerçant une ponction écologique sur les ressources et } \\
\text { qu'elles sont donc jugées incompatibles avec une protection efficace de l'environnement. } \\
\text { Lorsque de telles utilisations ne peuvent pas être gérées activement dans une aire de } \\
\text { catégorie II pour garantir que les objectifs généraux de la protection de l'écosystème sont } \\
\text { atteints, il faut se demander s'il faut encore autoriser quelque prélèvement que ce soit ou } \\
\text { si les objectifs pour la réserve, ou pour la zone dans la réserve, ne correspondent pas } \\
\text { mieux à une autre catégorie (p.ex. catégorie V ou VI) et ne devraient pas être changés. La } \\
\text { conservation de la nature dans des aires de catégorie II de l'environnement marin doit être } \\
\text { possible par la protection et ne pas exiger de gestion active importante ni de manipulation } \\
\text { de l'habitat. }\end{array}$ \\
\hline III & $\begin{array}{l}\text { La protection des monuments naturels ou d'éléments naturels dans des environnements marins } \\
\text { peut servir une variété d'objectifs. La protection localisée d'éléments comme des montagnes } \\
\text { sous-marines a une valeur de conservation importante, tandis que d'autres éléments marins } \\
\text { (comme les paysages historiques /archéologiques immergés) peuvent avoir une valeur culturelle } \\
\text { ou récréative pour des groupes particuliers, La catégorie III semble devoir être une désignation } \\
\text { relativement peu commune dans les écosystèmes marins. }\end{array}$ \\
\hline
\end{tabular}


Tableau 9. Application des catégories dans les aires marines protégées (suite)

\begin{tabular}{|c|c|}
\hline Catégorie & Notes relatives à l'utilisation dans les AMP \\
\hline IV & $\begin{array}{l}\text { Les aires de catégorie IV dans des environnements marins devraient jouer un rôle important } \\
\text { dans la protection de la nature et la survie des espèces (intégrant, lorsque c'est approprié, des } \\
\text { zones de reproduction, de frai, de nourrissage) ou d'autres éléments essentiels au bien-être } \\
\text { de la flore importante au niveau local ou national, ou de la faune résidente ou migratrice. La } \\
\text { catégorie IV est destinée à la protection d'espèces ou d'habitats particuliers, souvent avec une } \\
\text { intervention active de la gestion (p.ex. protection d'habitats benthiques clés contre la pêche } \\
\text { au chalut et le dragage). Les régimes de protection destinés à des espèces ou à des groupes } \\
\text { particuliers, là où d'autres activités ne sont pas limitées, seront souvent classifiés dans la } \\
\text { catégorie IV, p.ex. les sanctuaires des baleines. La protection limitée dans le temps, comme } \\
\text { dans le cas d'interdiction saisonnières de la pêche ou de protection de plages pour la ponte } \\
\text { des tortues pendant la saison de reproduction, peut aussi se qualifier pour la catégorie IV. } \\
\text { Contrairement au milieu terrestre où la catégorie IV peut inclure des fragments d'écosystèmes, } \\
\text { dans l'environnement marin, l'utilisation de cette catégorie offre une opportunité significative } \\
\text { de conserver l'écosystème sur une grande échelle, le plus souvent en englobant des zones de } \\
\text { catégorie la ou Ib ainsi que les intérêts liés à la catégorie II. }\end{array}$ \\
\hline V & $\begin{array}{l}\text { L'interprétation du concept de paysage marin dans les aires protégées suscite un intérêt } \\
\text { croissant. Les aires protégées de catégorie } V \text { soulignent l'importance de l'« interaction de } \\
\text { l'homme et de la nature dans le temps » et, dans un contexte marin, on peut généralement } \\
\text { s'attendre à ce que la catégorie } V \text { se retrouve typiquement dans des aires côtières. Le maintien } \\
\text { d'anciennes pratiques locales de pêche durable ou la collecte durable dans un récif corallien, } \\
\text { éventuellement dans des habitats côtiers culturellement modifiés (p.ex. par la plantation de } \\
\text { cocotiers), peut donner une mosaïque appropriée de modes de gestion qui peut se qualifier pour } \\
\text { la catégorie V. }\end{array}$ \\
\hline VI & $\begin{array}{l}\text { Les AMP qui conservent des habitats en grande partie naturels mais qui autorisent la récolte } \\
\text { d'éléments particuliers, comme certaines espèces comestibles ou de petites quantités de corail } \\
\text { ou de coquillages pour la vente aux touristes peuvent être identifiées comme de catégorie VI. } \\
\text { Il est parfois difficile de juger quand une aire gérée pour l'extraction de ses ressources devient } \\
\text { une aire protégée de catégorie } \mathrm{VI} \text {, et ceci sera déterminé, en fin de compte, par le fait de savoir } \\
\text { si l'aire répond à la définition générale d'une aire protégée et aussi si l'aire peut se justifier d'une } \\
\text { durabilité écologique vérifiable et mesurée au moyen d'indicateurs appropriés. }\end{array}$ \\
\hline
\end{tabular}

L'étendue des activités extractive et la rigueur de leur régulation sont des données importantes lorsqu'il faut déterminer la catégorie de l'UICN appropriée pour une AMP (ou une zone dans une AMP). Une utilisation extractive, y compris tout type de pêche, n'est pas conciliable avec les objectifs des catégories Ia et $\mathrm{Ib}$, et n'est probablement pas conciliable non plus avec la catégorie II.

6. Classer les AMP selon ce qu'elles font et non par l'appellation de la catégorie

L'assignation d'une AMP à une catégorie de l'UICN doit se baser sur l'examen des objectifs de gestion plutôt que sur le nom des catégories. Le même nom ou titre pour une AMP peut signifier des choses différentes dans des pays différents. Par exemple, le terme "sanctuaire ", utilisé dans le contexte des Etats-Unis, est une AMP à usage multiple désignée dans le cadre du National Marine Sanctuary Program (p.ex. le Florida Keys National Marine Sanctuary). Mais "sanctuaire " a une toute autre signification ailleurs - au Royaume-Uni, le terme est utilisé pour des réserves marines strictement protégées dans lesquelles toute utilisation extractive est interdite.
Tout comme pour les aires protégées terrestres et aquatiques intérieures, les catégories sont indépendantes du nom dans les AMP.

\section{Aires protégées d'eaux intérieures}

Les écosystèmes d'eaux intérieures n'occupent qu'une petite surface de la planète mais ce sont peut-être, parmi tous les biomes et habitats, ceux qui sont les plus lourdement touchés et menacés par les activités humaines. Des gouvernements et la communauté de la conservation se sont engagés à conserver des espèces et des habitats des eaux intérieures équivalents à ceux des milieux marins et terrestres, mais ces engagements doivent encore être largement concrétisés. De plus, la préservation de ces habitats de qualité fournit un service critique aux gens qui sont confrontés à des pénuries d'eau de plus en plus graves. L'attention aux eaux intérieure doit donc être intégrée à la gestion de toutes les aires protégées concernées, qui ellesmêmes doivent être gérées dans le respect du contexte de toute la biorégion et du bassin versant. 


\section{Définitions : Eaux intérieures, systèmes d'eau douce et zones humides}

Les termes eaux intérieures (zones humides intérieures), systèmes d'eau douce et, simplement, zones humides sont souvent utilisés de façon interchangeable, mais il y a quelques différences. Les eaux intérieures ou les zones humides intérieures font référence à tous les systèmes aquatiques non marins, y compris les salines intérieures et les systèmes d'eau saumâtre ; quant à savoir si des systèmes de transition comme les estuaires y sont inclus, c'est une question d'interprétation. Eaux intérieures sont les termes utilisés par la CDB. L'eau douce se définit techniquement comme " d', relatif à, vivant en, ou composé d'eau qui n'est pas salée ". Techniquement donc, cela exclut les salines et les systèmes intérieurs d'eau saumâtre, mais en pratique, les termes sont souvent utilisés dans le même sens que zones humides intérieures. La Convention de Ramsar définit les zones humides comme "étendues de marais, de fagnes, de tourbières ou d'eaux naturelles ou artificielles, permanentes ou temporaires, où l'eau est stagnante ou courante, douce, saumâtre ou salée, y compris des étendues d'eau marine dont la profondeur à marée basse n'excède pas six mètres ". Dans certaines régions du monde, on considère, de façon informelle, que les termes zones humides excluent les systèmes aquatiques qui ne sont pas couverts de végétation, tels des cours d'eau, des lacs et des nappes phréatiques. Dans le contexte de ces lignes directrices, nous utilisons les termes eaux intérieures pour décrire la variété d'habitats aquatiques et semi-aquatiques, et les espèces associées, qui ne rentrent pas dans les classifications marines. Les zones humides naturelles intérieures incluent (modifié d'après l'Evaluation des écosystèmes pour le Millénaire, Rapport de synthèse sur les zones humides et l'eau, Tableau 3.1) :

- Les rivières et les cours d'eau permanents et temporaires :

- Les lacs permanents ;

- Les lacs, les marais et les marécages saisonniers, y compris les plaines inondables ;

- Les zones humides alpines et de toundra ;

- Les sources, les oasis et les zones humides géothermiques ;

- Les zones humides souterraines, y compris les grottes et les systèmes d'eau souterrains.

\section{Complexité de la protection des eaux intérieures}

La relation entre la protection des aires protégées et celle des eaux intérieures est complexe. Il existe de nombreuses incompatibilités réelles ou ressenties, et les défis qui apparaissent lorsqu'on examine cette relation comprennent les aspects suivants :
- Relation et rôle du paysage. Les systèmes d'eaux intérieures font partie du paysage terrestre plus global, dont certaines portions spécifiques en amont sont liées à leur bassin versant ${ }^{10}$ par toute une variété de processus hydrologiques superficiels ou souterrains. L'idée de " clôturer " des systèmes de zones humides est, dans la plupart des cas, irréalisable pour les raisons données plus haut. Les aires protégées les plus efficaces pour la conservation des eaux intérieures sont celles qui font partie d'une gestion intégrée de bassin hydrographique, parfois appelée gestion intégrée de l'eau par bassin versant (GIEBV). Celle-ci implique une stratégie à l'échelle du paysage pour atteindre dans le même temps des objectifs environnementaux, économiques et sociaux. La GIEBV est une forme d'approche par écosystème que les Etats Parties à la $\mathrm{CDB}$ se sont engagés à mettre en œuvre. Les gouvernements mondiaux se sont aussi engagés à planifier et à réaliser une gestion intégrée des ressources en eau (GIRE) qui est semblable, en théorie, à la GIEBV mais qui n'est pas liée géographiquement à des bassins de rivières. En pratique, malheureusement, la GIRE et même la GIEBV n'ont pas toujours accordé l'attention nécessaire à la conservation de la biodiversité des eaux intérieures.

- Processus écologiques. Le " moteur clé " des systèmes (lotiques) d'eaux vives est le régime du débit $:^{11}$ l'importance, la fréquence, le temps, la durée et le taux de changement du flux d'eau. Dans des systèmes (lentiques) d'eau stagnante, la variable principale est généralement l'hydropériode : le schéma saisonnier et cyclique de l'eau. Le débit et l'hydropériode caractérisent le « schéma hydrologique " d'un système. Pour presque tous les systèmes d'eaux intérieures, l'eau est générée " en dehors " des systèmes eux-mêmes et entre par des voies de surface et souterraines et par des affluents. La protection du schéma hydrologique requiert donc une protection ou une gestion qui s'étend vers l'amont et plus haut en altitude et souvent aussi jusqu'aux nappes phréatiques. ${ }^{12}$ Dans de nombreux cas, il peut être nécessaire de faire une gestion transfrontalière de l'eau, même si l'aire protégée en question se trouve dans un seul état. Dans le cas de la plupart des aires protégées existantes, cela se traduit par un travail avec des parties prenantes et des partenaires pour gérer les régimes de débit en dehors des aires protégées.

- Connectivité longitudinale. Les cours d'eau et leurs réseaux ont une dimension linéaire, ou longitudinale, mais aussi une dimension latérale, verticale et temporelle. La protection de la connectivité longitudinale - le lien entre

10 Un bassin versant est défini ici comme toutes les terres encloses dans une ligne continue hydrologiquement close qui se situe en amont d'un point spécifique d'un cours d'eau ; ou, dans le cas de bassins fermés, c'est toute la surface topographique drainée par ce cours d'eau.

11 Le débit est le volume d'eau qui passe à un point donné par unité de temps.

12 L'équivalent souterrain d'un bassin versant, ou aire de captage en surface. 
des habitats, des espèces, des communautés et des processus écologiques entre les portions amont et aval d'un cours d'eau ou d'un réseau formant corridor - est souvent un but essentiel de la conservation des eaux intérieures et elle implique d'empêcher ou d'enlever les barrières physiques ou chimiques. La protection de la connectivité longitudinale est aussi identifiée comme critique pour préserver des systèmes résilients face aux changements climatiques. Inversement, une connectivité supplémentaire artificielle, comme cela se passe lors de transferts entre bassins, peut être délétère à cause de l'invasion d'espèces exotiques. Des aires protégées traditionnelles sont souvent considérées comme des éléments polygonaux plutôt que linéaires, et elles sont rarement conçues en vue de la protection et de la gestion de la connectivité longitudinale des cours d'eau. Souvent, le tracé des cours d'eau sert à marquer les limites des aires protégées sans recevoir lui-même de protection propre.

- Connectivité latérale. Les connexions latérales entre les cours d'eau et le paysage environnant sont essentielles pour la santé écologique des cours d'eau et celle des plaines inondables et des communautés riveraines associées. Ces connexions dépendent en grande partie des processus hydrologiques décrits plus haut; avec l'interaction entre le débit du courant et les terres riveraines qui créent les conditions dynamiques, elles sont la base des habitats riches et uniques des plaines inondables et des zones humides riveraines. Ces terres contribuent aussi à l'apport de matériel organique et inorganique dans les cours d'eau et peuvent préserver les habitats aquatiques contre les polluants. La largeur de ces aires varie fortement, de bandes relativement étroites aux endroits où les berges sont escarpées à des plaines inondables extrêmement larges. Les aires protégées peuvent jouer un rôle important dans la conservation des habitats riverains et inondables et de leur connectivité avec les lits des cours d'eau.

- Interactions eaux souterraines-eaux de surface. La protection des espèces vivant dans les eaux intérieures de surface exige d'étudier l'hydrologie sous la surface. Les systèmes alimentés par les nappes phréatiques sont fréquents dans de nombreuses aires, ce qui exige de protéger les cours souterrains aussi bien que les eaux de surface. La plupart des eaux de surface dépendent aussi des eaux souterraines (la nappe phréatique) pour leur fonctionnement, qu'elles soient, ou non, alimentées par ces dernières. Les eaux souterraines, comme dans les aires karstiques, offrent un habitat à des espèces souvent spécialisées mais aussi de l'eau à des millions de personnes. Les bassins versants phréatiques et les zones de captage en surface peuvent ne pas coïncider dans l'espace ou la géopolitique, ce qui ajoute un niveau de complexité à la protection des eaux courantes.

- Menaces exogènes. Les eaux intérieures se trouvent généralement aux endroits les plus bas du paysage et elles reçoivent par conséquent des perturbations propagées dans les bassins et transmises par l'eau (p.ex. la pollution, l'érosion du sol et l'eutrophisation). S'il est vrai que toutes les aires protégées doivent affronter des menaces provenant de l'extérieur de leurs limites, celles qui protègent des systèmes d'eaux intérieures doivent expressément se préoccuper de menaces en amont, en altitude et dans certains cas même en aval (comme les espèces invasives).

- Exclusion de ressources d'eaux intérieures. Des communautés humaines se sont toujours installées à proximité des systèmes d'eaux intérieures qui leur fournissent une vaste gamme de services écosystémiques essentiels. Le droit fondamental d'accéder à l'eau douce, à l'intérieur ou en amont des aires protégées, peut entrer en conflit avec les objectifs de certaines catégories d'aires protégées qui limitent l'utilisation des ressources par les hommes.

- Autorités de gestion multiples. Dans beaucoup de pays, si pas la plupart d'entre eux, les responsabilités de différentes agences gouvernementales peuvent se superposer et éventuellement entrer en conflit lorsqu'elles concernent la gestion des ressources en eau douce, des espèces des zones humides, des habitats aquatiques, des paysages environnants et des aires protégées. Par conséquent, la gestion d'espèces et d'habitats d'eaux intérieures au sein d'une aire protégée - ce qui, comme on l'a noté plus haut, va vraisemblablement exiger de gérer des terres et de l'eau aussi en dehors de l'aire protégée - peut être compliquée par le besoin de coordonner les activités entre de multiples autorités, dont certaines ont des mandats tout à fait étrangers à la conservation de la biodiversité.

En bref, les défis abondent. Si, idéalement, les aires protégées établies pour préserver des écosystèmes d'eaux intérieures vont englober des bassins entiers, il faudra généralement appliquer des combinaisons novatrices d'aires protégées et d'autres stratégies dans le cadre d'une GIEBV. Les aires protégées existantes, désignées et conçues pour protéger des écosystèmes terrestres, conferent sans doute certains bénéfices à la biodiversité de zone humide grâce à la gestion par paysage, mais il existe de sérieuses opportunités d'assurer une meilleure protection. La conception de nouvelles aires protégées peut, et doit, inclure dès le début une attention pour les eaux intérieures afin de leur assurer une meilleure intégration. Les pages suivantes présentent des lignes directrices préliminaires sur la façon dont la gamme des différentes approches de gestion dans les aires protégées, représentées par les catégories, peut aider à une meilleure conservation des zones humides intérieures.

\section{Appliquer la nouvelle définition d'une aire protégée}

La nouvelle définition d'une aire protégée - Un espace géographique clairement défini, reconnu, consacré et géré, par tout moyen efficace, juridique ou autre, pour assurer à long terme la 
conservation de la nature ainsi que les services écosystémiques et les valeurs culturelles qui lui sont associés - prend mieux en compte les eaux douces que la définition adoptée précédemment grâce au remplacement de "une portion de terre etlou de mer " par " un espace géographique clairement défini ». Les aires protégées qui, au départ, préservent des éléments d'eaux intérieures, comme des rivières corridors ou des lacs, sont maintenant clairement couvertes par la définition. Cela inclut certains types d'aires protégées qui sont uniques dans les écosystèmes d'eaux intérieures, comme les cours d'eau non aménagés. ${ }^{13}$ Une large gamme de stratégies de conservation qui visent à protéger la quantité et la qualité de l'eau, par exemple en gérant les flux environnementaux ${ }^{14}$ et en appliquant des pratiques de gestion rationnelles à l'utilisation des sols, n'entrent normalement pas dans la définition d'une aire protégée. Elles sont mentionnées ici parce que la conservation efficace des systèmes d'eaux intérieures dans les aires protégées n'est atteinte, dans la plupart des cas, que par l'utilisation coordonnée de ces stratégies au-delà des limites des aires protégées.

\section{Appliquer les catégories d'aires protégées}

N'importe quelle catégorie peut, en principe, s'appliquer à des aires qui ont des objectifs implicites de conservation des zones humides. Des exemples d'aires protégées qui ont des objectifs clairs liés à la conservation de zones humides intérieures peuvent se trouver dans chaque catégorie de l'UICN (Tableau 10) :

Tableau 10. Exemples d'aires protégées de différentes catégories fournissant des bénéfices aux eaux intérieures

\begin{tabular}{|c|c|c|}
\hline Catégorie & Exemple & Description \\
\hline la & $\begin{array}{l}\text { Srebarna } \\
\text { Nature Reserve } \\
\text { (Bulgarie) }\end{array}$ & $\begin{array}{l}\text { Une réserve de biosphère de } 600 \text { ha, site du patrimoine mondial (SPM), et site } \\
\text { Ramsar, pour protéger le lac Srebarna dans la plaine inondable du Danube. La } \\
\text { réserve a été créée au départ pour protéger la riche avifaune, et spécialement les } \\
\text { oiseaux d'eau. }\end{array}$ \\
\hline $\mathrm{Ib}$ & $\begin{array}{l}\text { Avon } \\
\text { Wilderness } \\
\text { Park (Australie) }\end{array}$ & $\begin{array}{l}\text { Un parc de nature sauvage de } 39.650 \text { ha couvrant tous les bassins versants des } \\
\text { sources de la rivière Avon, désigné pour la conservation et les loisirs autonomes } \\
\text { selon la Loi sur les parcs nationaux. }\end{array}$ \\
\hline II & $\begin{array}{l}\text { Pantanal } \\
\text { National Park } \\
\text { (Brésil) }\end{array}$ & $\begin{array}{l}\text { Un parc national (et site Ramsar) de } 135.000 \text { ha situé dans une large dépression } \\
\text { fonctionnant comme un delta intérieur. L'aire consiste en une vaste région de } \\
\text { savanes inondées saisonnièrement, d'îlots de broussailles xérophytiques et de } \\
\text { forêt humide décidue. }\end{array}$ \\
\hline III & $\begin{array}{l}\text { Lac Ganga } \\
\text { (Mongolie) }\end{array}$ & $\begin{array}{l}\text { Un élément naturel (et site Ramsar) de } 32.860 \text { ha englobant un petit lac saumâtre } \\
\text { et des lacs associés, dans l'Est de la Mongolie, dans un paysage unique } \\
\text { combinant zones humides, steppes et dunes de sable. Le district du lac est très } \\
\text { important pour les oiseaux d'eau reproducteurs et de passage. }\end{array}$ \\
\hline IV & $\begin{array}{l}\text { Koshi Tappu } \\
\text { (Népal) }\end{array}$ & $\begin{array}{l}\text { Une réserve de faune de } 17.500 \text { ha sise le long de la rivière Sapta Kosi et } \\
\text { composée de vasières et de marais frangeants. La réserve abrite la dernière } \\
\text { population de buffles d'eau sauvages du Népal. }\end{array}$ \\
\hline V & $\begin{array}{l}\text { Big South Fork } \\
\text { (USA) }\end{array}$ & $\begin{array}{l}\text { Cette « rivière et zone de loisirs nationale » occupe } 50.585 \text { ha sur le plateau de } \\
\text { Cumberland et protège la Big South Fork non aménagée de la rivière Cumberland et } \\
\text { ses affluents. L'aire a été en grande partie protégée pour ses opportunités récréatives. }\end{array}$ \\
\hline VI & Titicaca (Pérou) & $\begin{array}{l}\text { Une réserve nationale de } 36.180 \text { ha créée pour protéger le lac navigable le plus } \\
\text { haut du monde. }\end{array}$ \\
\hline
\end{tabular}

Les eaux intérieures peuvent être zonées pour permettre différents degrés d'utilisation. Par exemple, dans le Parc National du Lac Malawi, au Malawi, les méthodes de pêche traditionnelles destinées à pêcher des poissons migrateurs sont autorisées dans des aires délimitées alors que dans la plus grande partie du parc, les poissons locaux ne peuvent pas être pêchés.

Savoir si et comment les catégories d'aires protégées sont liées aux mesures de protection se fait au cas par cas. Le
Tableau 11 donne la liste d'un certain nombre de stratégies locales et identifie quand elles sont particulièrement compatibles, pas incompatibles ou incompatibles avec les catégories d'aires protégées de l'UICN. Ces assignations sont des généralités, et il existe des exceptions. Des sites du patrimoine mondial, des sites Ramsar et des réserves de biosphère sont inclus parce qu'ils sont largement utilisés pour protéger des éléments des eaux intérieures et parce qu'ils ont fait du zonage un instrument de gestion.

13 Rivières sauvages et rivières pittoresques sont couvertes par des législations différentes dans certains pays.

14 La qualité, la quantité et le timing des flux d'eau requis pour préserver les composantes, les fonctions, les processus et la résilience des écosystèmes aquatiques qui fournissent aux gens des biens et des services. 
Tableau 11. Compatibilité de diverses stratégies de protection des eaux intérieures avec les catégories de l'UICN

\begin{tabular}{|c|c|c|c|c|c|c|c|c|c|}
\hline \multirow{2}{*}{$\begin{array}{l}\text { Type d'aire protégée : } \\
\text { les descriptions font } \\
\text { normalement référence à ces } \\
\text { types comme à des entités } \\
\text { isolées - tous peuvent } \\
\text { être intégrés comme des } \\
\text { portions de plus grandes } \\
\text { réserves }\end{array}$} & \multicolumn{7}{|c|}{$\begin{array}{l}\text { Compatibilité avec la catégorie } \\
\text { de l'aire protégée }\end{array}$} & \multirow{2}{*}{$\begin{array}{l}\text { Si l'aire se trouve } \\
\text { en dehors de I-VI, } \\
\text { probabilité de sa } \\
\text { contribution à } \\
\text { la conservation } \\
\text { dans la GIEBV* }\end{array}$} & \multirow{2}{*}{ Exemples } \\
\hline & la & $\mathrm{lb}$ & II & III & IV & V & VI & & \\
\hline \multicolumn{10}{|c|}{ Désignation/reconnaissance par une convention ou un programme international } \\
\hline Site du patrimoine mondial & & & & & & & & Faible & Lac Malawi (Malawi) \\
\hline Site Ramsar & & & & & & & & Très forte & $\begin{array}{l}\text { Upper Navua Conservation } \\
\text { Area (Fidji) }\end{array}$ \\
\hline Réserve de biosphère & & & & & & & & Forte & Lac Dalai (Chine) \\
\hline \multicolumn{10}{|c|}{ Mécanismes locaux de protection de l'eau douce } \\
\hline Rivière non aménagée & & & & & & & & Forte & Upper Delaware River (USA) \\
\hline Réserve/tampon riverain & & & & & & & & Forte & $\begin{array}{l}\text { Douglas River/Daly River } \\
\text { Esplanade Conservation } \\
\text { Area (Australie) }\end{array}$ \\
\hline Réserve de plaine inondable & & & & & & & & Forte & Pacaya-Samiria (Pérou) \\
\hline Réserve de pêche/récolte & & & & & & & & Forte & Lubuk Sahab (Indonésie) \\
\hline $\begin{array}{l}\text { Réserve de zone humide } \\
\text { pour la faune/chasse }\end{array}$ & & & & & & & & Limitée & $\begin{array}{l}\text { Ndumo Game Reserve } \\
\text { (Afrique du Sud) }\end{array}$ \\
\hline $\begin{array}{l}\text { Aire récréative réservée à la } \\
\text { pêche }\end{array}$ & & & & & & & & Limitée & Onon River (Mongolie) \\
\hline $\begin{array}{l}\text { Zone protégée de captage } \\
\text { d'eau }\end{array}$ & & & & & & & & Forte & $\begin{array}{l}\text { Rwenzori Mountains } \\
\text { National Park (Ouganda) }\end{array}$ \\
\hline $\begin{array}{l}\text { Zone de recharge d'aquifère } \\
\text { protégé }\end{array}$ & & & & & & & & Forte & Susupe Wetland (Saipan) \\
\hline \multicolumn{10}{|c|}{ Autres mécanismes locaux avec bénéfices potentiels de l'eau douce } \\
\hline $\begin{array}{l}\text { Réserve marine/zone de } \\
\text { gestion côtière }\end{array}$ & & & & & & & & Faible & $\begin{array}{l}\text { Delta du Danube } \\
\text { (Roumanie) }\end{array}$ \\
\hline $\begin{array}{l}\text { Pêcherie fermée } \\
\text { saisonnièrement }\end{array}$ & & & & & & & & Limitée & Lac Santo Antonio (Brésil) \\
\hline Réserve forestière & & & & & & & & Limitée & $\begin{array}{l}\text { Forêts préservées des } \\
\text { Sundarbans (Bangladesh) }\end{array}$ \\
\hline Zone de forêt certifiée & & & & & & & & Limitée & Upper St. John River (USA) \\
\hline
\end{tabular}

Particulièrement compatible avec la catégorie d'aire protégée

Pas incompatible avec la catégorie d'aire protégée

Pas particulièrement ou jamais compatible avec la catégorie d'aire protégée
${ }^{*}$ GIEBV = gestion intégrée de l'eau par bassin versant 
Les aires protégées créées totalement ou partiellement pour protéger les eaux intérieures, y compris la plupart des sites Ramsar, ne sont pas toutes assignées à une catégorie. De plus, de nombreuses aires protégées qui contribuent à la conservation d'un écosystème d'eaux intérieures n'ont pas de statut Ramsar. Par conséquent, il n'est actuellement pas possible d'évaluer mondialement quelles aires protégées existantes ont des objectifs qui concernent les eaux intérieures, ni comment les catégories de l'UICN y ont été appliquées. Différents types de systèmes d'eaux intérieures, qui se trouvent dans un état plus ou moins naturel, peuvent se prêter davantage à certaines catégories d'aires protégées qu'à d'autres : le Tableau 12 fait quelques suggestions.

\section{Protection intégrée des systèmes terrestres et de zones humides intérieures}

Il est souvent difficile d'identifier une " aire protégée d'eaux intérieures ", et l'influence d'une aire protégée sur les systèmes aquatiques peut avoir autant à voir avec les objectifs de sa gestion qu'avec les habitats qui la composent. Des aires marines protégées sont facilement identifiées par leur localisation. Les systèmes d'eaux intérieures, cependant, s'étendent sur tout le paysage terrestre et se trouvent dans pratiquement toutes les aires protégées terrestres. Certaines aires protégées, comme les rivières non aménagées et de nombreux sites Ramsar, peuvent clairement être qualifiées d' " aires protégées d'eaux

Tableau 12. Les catégories d'aires protégées les plus appropriées pour différents types d'écosystèmes d'eaux intérieures

\begin{tabular}{|c|c|c|c|c|c|c|c|c|}
\hline \multirow{2}{*}{$\begin{array}{l}\text { Type d'écosystème d'eau } \\
\text { douce }\end{array}$} & \multicolumn{7}{|c|}{ Catégorie UICN } & \multirow[t]{2}{*}{ Exemples } \\
\hline & la & $\mathrm{lb}$ & II & III & IV & V & VI & \\
\hline \multicolumn{9}{|l|}{ Cours d'eau } \\
\hline Bassin versant complet & & & & & & & & Kakadu National Park (Australie) \\
\hline $\begin{array}{l}\text { Cours d'eau complet ou } \\
\text { portion substantielle }\end{array}$ & & & & & & & & Fraser Heritage River (Canada) \\
\hline Sources supérieures & & & & & & & & Adirondack Forest Reserve (Etats-Unis) \\
\hline Portions moyennes et basses & & & & & & & & Parc national de Doñana (Espagne) \\
\hline Zones riveraines & & & & & & & & $\begin{array}{l}\text { Douglas River/Daly River Esplanade Conservation } \\
\text { Area (Australie) }\end{array}$ \\
\hline Sections de lits de rivière & & & & & & & & Hippo Pool National Monument (Zambie) \\
\hline Gorges & & & & & & & & Fish River Canyon Conservation Area (Namibie) \\
\hline Chutes d'eau & & & & & & & & Parc National d'Iguaçu (Argentine/Brésil) \\
\hline
\end{tabular}

\section{Zones humides et lacs}

Zones humides à plaine inondable

\section{Lacs}

Portions de lacs

Deltas intérieurs

Deltas côtiers

Zones humides côtières

Zones humides géothermiques

Sources

Zones humides alpines et de toundra

Marais d'eau douce

Tourbières

\section{Zones humides souterraines}

Eaux et grottes karstiques
Mamirauá Sustainable Development Reserve (Brésil)

Lac Balaton (Hongrie)

Rubondo Island National Park (Tanzanie)

Okavango Delta Wildlife Management Area (Botswana)

Réserve de biosphère du Delta du Danube (Roumanie)

Parc national de Doñana (Espagne)

Lac Bogoria (Kenya)

Ash Meadows National Wildlife Refuge (USA)

Bitahai Wetland (Chine)

Busanga Swamps (Zambie)

Silver Flowe National Nature Reserve (UK) 
intérieures ", mais la désignation d'autres sites peut être ambiguë. Certaines ont inclus dès le départ des objectifs de gestion terrestres et d'eau douce, alors que d'autres qui étaient conçues au début pour protéger des éléments terrestres en sont venues avec le temps à incorporer des objectifs concernant l'eau douce. Le Parc National Kruger, en Afrique du Sud, en est un exemple : il fut créé à l'origine pour protéger sa faune de grands mammiferes, mais on estime que les zones fluviales et riveraines supportent 50 pour cent du biote du parc, et la gestion consacre aujourd'hui quelque 30 pour cent de son effort de gestion aux eaux intérieures.

Même si certaines aires protégées sont un avantage pour les systèmes de zones humides intérieures qui s'y trouvent, il existe de nombreux exemples où ce n'est pas le cas. Dans de nombreux cas, les écosystèmes de zones humides intérieures sis dans les aires protégées ont été délibérément altérés pour fournir de l'eau ou de l'électricité, et même pour faciliter l'observation de la faune ou à d'autres fins récréatives. Intégrer une attention spéciale aux zones humides intérieures dans la gestion de toutes les aires protégées concernées est indispensable, y compris dans les AMP côtières. La gestion des aires protégées terrestres pourrait mieux se préoccuper des eaux intérieures, par exemple en :

- Protégeant ou en restaurant la connectivité longitudinale et latérale des cours d'eau corridors (p.ex. en enlevant des obstacles, en reconnectant rivières avec plaines inondables, en s'assurant que les routes et les infrastructures associées ne fragmentent pas les systèmes des cours d'eau) ;

- Protégeant la faune indigène (p.ex. en empêchant l'introduction de poissons exotiques ou la surpêche) ;

- Protégeant la flore indigène - particulièrement dans des zones riveraines qui peuvent être négligées dans le reste de l'aire protégée ;

- Gérant les activités récréatives aquatiques (p.ex. par la restriction d'accès aux engins motorisés et des déversements associés) ;

- Protégeant agressivement la qualité de l'eau (p.ex. gestion méticuleuse à la source des déchets dans les installations récréatives) ;

- Protégeant l'écoulement des sources pour que les utilisateurs situés en aval profitent des bénéfices des services écosystémiques ;

- Protégeant ou en restaurant les zones tampons riveraines dans un parc et le long de sa limite si une rivière en détermine le tracé (et élargir les limites de l'aire protégée quand c'est possible en utilisant les critères appropriés aux écosystèmes de zones humides intérieures - p.ex. se basant sur les limites du bassin versant et pas sur le tracé du lit de la rivière pour délimiter les aires) ;

- Protégeant spécialement les sources ou les bassins sacrés qui ont une importance culturelle.
En raison, partiellement, de l'ambiguïté persistante quant à savoir si une aire est une " aire protégée d'eaux intérieures ", la séparation de ces composantes dans les processus de comptesrendus, comme à la WDPA, reste un vrai défi. Il peut être difficile de mesurer et d'interpréter la taille de nombreuses zones humides - dans de nombreux cas, les zones humides varient beaucoup à cause de facteurs naturels (p.ex. inondations saisonnières) - et actuellement, la WDPA ne prévoit pas de mesures de longueur. Tant que la conservation des eaux intérieures n'est pas intégrée plus efficacement dans les plans de gestion des aires protégées et que ces plans de gestion ne reconnaissent pas les processus et les menaces extérieures sur les limites des aires protégées, l'étendue géographique des systèmes d'eaux intérieures au sein des aires protégées nous en dit plus sur le potentiel de conservation que sur la conservation elle-même.

\section{Sites naturels sacrés}

Les sites sacrés (qui comprennent les sites et les paysages naturels sacrés) qui correspondent aux définitions nationales et internationales des aires protégées peuvent être reconnus comme des composantes légitimes des systèmes d'aires protégées et peuvent être assignés à l'une des six catégories d'aires protégées de l'UICN. En même temps, les valeurs culturelles et spirituelles des aires protégées pourraient être mieux reflétées dans toute la gamme des catégories où elles sont actuellement absentes ou insuffisamment reconnues.

De nombreuses aires protégées contiennent des sites importants pour une ou parfois plusieurs croyances religieuses ou pour des systèmes de valeurs spirituelles, y compris des sites naturels sacrés et des monuments architecturaux comme des monastères, des temples, des lieux et des sentiers de pèlerinage. Même dans les systèmes d'aires protégées des pays les plus sécularisés d'Europe, qui ont été établis seulement sur des critères écologiques, on estime que 20 à 35 pour cent d'entre eux incluent d'importantes valeurs culturelles ou spirituelles. Il existe des pays et des territoires où toute la nature est sacrée, et les aires protégées peuvent y former de plus petites entités dans des paysages sacrés. Les gestionnaires doivent garantir que ces valeurs spirituelles sont protégées au même titre que le patrimoine naturel. Pourtant, les sites sacrés ne sont actuellement pas bien reflétés dans la désignation et dans les plans de gestion des aires protégées, et les politiques et cadres juridiques existants n'appuient pas correctement les sites (naturels) sacrés. Il est évident pour beaucoup que les sites naturels sacrés représentent une protection efficace de la biodiversité, parfois depuis des siècles. Les sites sacrés peuvent se trouver dans des écosystèmes plus ou moins naturels, dans des paysages culturels ou des paysages gérés, et lorsqu'ils se situent dans des aires protégées, ils doivent être complètement intégrés dans les stratégies de gestion, en coopération avec les groupes religieux ou communautaires concernés. Le Tableau 13 en donne quelques exemples. 
Tableau 13. Exemples de sites sacrés dans les catégories de l'UICN

\begin{tabular}{|l|l|l|l|}
\hline la & \multicolumn{3}{|l}{ Réserve naturelle intégrale : aire protégée gérée principalement à des fins scientifiques } \\
\hline & Sri Lanka & Parc National de Yala & $\begin{array}{l}\text { Important pour les bouddhistes et les hindous, demande un haut } \\
\text { niveau de protection pour des raisons religieuses. }\end{array}$ \\
\cline { 2 - 4 } $\begin{array}{l}\text { Fédération } \\
\text { de Russie }\end{array}$ & Yuganskiy Kanthy & $\begin{array}{l}\text { Important pour les chrétiens. L'aire protégée a été créée autour du } \\
\text { lac Numto - un endroit sacré pour les Khanti et les Nénets - dans } \\
\text { la région de Beloïarsk. }\end{array}$
\end{tabular}

Ib Zone de nature sauvage : aire protégée gérée principalement à des fins de protection de la nature sauvage

\begin{tabular}{|l|l|l|}
\hline Mongolie & Bogd Khan Mountain & $\begin{array}{l}\text { La montagne est importante pour le bouddhisme et, avant, l'était } \\
\text { pour le shamanisme. Elle a été officiellement désignée par l'Etat } \\
\text { comme montagne sacrée. Il existe des preuves de la déclaration } \\
\text { comme aire de nature sauvage dès 1294. }\end{array}$ \\
\hline Mongolie & Dornod Mongol & $\begin{array}{l}\text { Important pour le bouddhisme. Vangiin Tsagaan Uul (Montagne } \\
\text { blanche de Vangi) est un pic sacré pour le bouddhisme à l'intérieur } \\
\text { de la réserve. }\end{array}$
\end{tabular}

II Parc National : aire protégée gérée principalement dans le but de protéger l'écosystème et à des fins récréatives

\begin{tabular}{|l|l|l|} 
Malawi & Nyika National Park & $\begin{array}{l}\text { Grande aire englobant quatre sites sacrés que la population locale } \\
\text { peut encore utiliser lors de cérémonies rituelles pour faire pleuvoir. }\end{array}$ \\
\hline Japon & $\begin{array}{l}\text { Parc nationaux et SPM } \\
\text { de Kii Mountains }\end{array}$ & $\begin{array}{l}\text { Plusieurs temples shintoïstes et bouddhistes, des sites sacrés et } \\
\text { des sentiers de pèlerinage pour les deux confessions, en usage } \\
\text { continu depuis plus d'un millénaire. }\end{array}$ \\
\hline Inde & $\begin{array}{l}\text { Parc National du Grand } \\
\text { Himalaya }\end{array}$ & $\begin{array}{l}\text { Inclut de nombreuses places d'importance religieuse pour } \\
\text { I'hindouisme. }\end{array}$
\end{tabular}
III Monument naturel : aire protégée gérée principalement dans le but de préserver des éléments naturels spécifiques

\begin{tabular}{|c|c|c|c|}
\hline & Cambodge & $\begin{array}{l}\text { Sanctuaire de la vie } \\
\text { sauvage de Phnom } \\
\text { Prich }\end{array}$ & $\begin{array}{l}\text { Une petite superficie dans le sanctuaire est une forêt sacrée et dès } \\
\text { lors un monument naturel (un autre exemple : les forêts Kaya du } \\
\text { Kenya). }\end{array}$ \\
\hline & $\begin{array}{l}\text { Fédération } \\
\text { de Russie }\end{array}$ & $\begin{array}{l}\text { La Montagne d'Or de } \\
\text { l'Altaï }\end{array}$ & $\begin{array}{l}\text { Sacrée pour les autochtones altaïens et de nombreuses } \\
\text { confessions différentes, y compris le Bouddhisme, le Christianisme } \\
\text { et l'Islam. }\end{array}$ \\
\hline & Grèce & $\begin{array}{l}\text { SPM de la Péninsule } \\
\text { du Mont Athos }\end{array}$ & $\begin{array}{l}\text { Un bastion de la chrétienté orthodoxe qui comprend } 20 \text { monastères } \\
\text { inclus dans un Etat monastique et des centaines de plus petits } \\
\text { monastères, ermitages et grottes qui comptent plus de } 1000 \text { ans } \\
\text { d'activité monastique ininterrompue. }\end{array}$ \\
\hline & Espagne & $\begin{array}{l}\text { Réserve naturelle } \\
\text { et Parc Naturel de } \\
\text { Montserrat }\end{array}$ & $\begin{array}{l}\text { Une montagne sainte hébergeant d'anciens ermitages et un } \\
\text { monastère chrétien qui sont un lieu de pèlerinage depuis le } 14 \text { ème } \\
\text { siècle. C'est aujourd'hui l'aire protégée la plus visitée d'Espagne. }\end{array}$ \\
\hline IV & \multicolumn{3}{|c|}{$\begin{array}{l}\text { Aire de gestion des habitats/espèces : aire protégée gérée principalement à des fins de conservation } \\
\text { avec intervention de la gestion }\end{array}$} \\
\hline & Liban & $\begin{array}{l}\text { SPM de la Vallée de la } \\
\text { Qadisha et les cèdres } \\
\text { du Seigneur }\end{array}$ & $\begin{array}{l}\text { Forêt sacrée pour l'Eglise chrétienne maronite qui comprend un } \\
\text { monastère important, des ermitages, et la résidence d'autorités } \\
\text { religieuses. }\end{array}$ \\
\hline & Bornéo & Jardins tembawang & $\begin{array}{l}\text { Certains sites sacrés auront besoin d'une intervention continue, ou } \\
\text { même d'être replantés, tels les jardins tembawang qui renferment } \\
\text { une grande biodiversité. }\end{array}$ \\
\hline & Sri Lanka & $\begin{array}{l}\text { Peak Wilderness Park, } \\
\text { (Sri Pada-Adams Peak) }\end{array}$ & $\begin{array}{l}\text { Site naturel sacré pour l'islam, le bouddhisme, l'hindouisme et } \\
\text { le christianisme, qui attirent de nombreux pèlerins de toutes ces } \\
\text { religions. }\end{array}$ \\
\hline
\end{tabular}


Tableau 13. Exemples de sites sacrés dans les catégories de l'UICN (suite)

\begin{tabular}{|c|c|c|c|}
\hline \multirow[t]{2}{*}{ V } & \multicolumn{3}{|c|}{$\begin{array}{l}\text { Paysage terrestre/marin protégé : aire protégée gérée principalement dans le but d'assurer la } \\
\text { conservation de paysages terrestres/marins et à des fins récréatives }\end{array}$} \\
\hline & Chine & $\begin{array}{l}\text { Parc National de } \\
\text { Xishuangbanna }\end{array}$ & $\begin{array}{l}\text { Paysage avec plusieurs sites sacrés (bosquets et montagnes) } \\
\text { qui sont depuis longtemps gérés par la communauté. }\end{array}$ \\
\hline & Roumanie & $\begin{array}{l}\text { Parc naturel de } \\
\text { Vanatori Neamt }\end{array}$ & $\begin{array}{l}\text { Le cœur spirituel de la Roumanie, qui comprend } 16 \text { monastères } \\
\text { chrétiens ainsi qu'une faune sauvage exceptionnelle : le bison } \\
\text { d'Europe, l'ours brun, et des populations de loups. }\end{array}$ \\
\hline V $\mathbf{l}$ & \multicolumn{3}{|c|}{$\begin{array}{l}\text { Aire protégée de ressources gérées : aire protégée gérée principalement à des fins d'utilisation } \\
\text { durable des écosystèmes naturels }\end{array}$} \\
\hline & Equateur & Cayapas Mataje & $\begin{array}{l}\text { Aire d'utilisation durable dont on dit qu'elle contient les plus hautes } \\
\text { mangroves du monde, et connue pour d'importants spirites révérés } \\
\text { par la population locale. }\end{array}$ \\
\hline & USA & $\begin{array}{l}\text { San Francisco Peaks } \\
\text { National Forest }\end{array}$ & Forêt sacrée pour plus d'une douzaine de tribus d'Amérindiens. \\
\hline & Egypte & $\begin{array}{l}\text { SPM de la Zone Ste } \\
\text { Catherine - Mt Sinaï }\end{array}$ & $\begin{array}{l}\text { Le Mont Sinaï est sacré pour le judaïsme, le christianisme et l'islam. } \\
\text { L'ancien monastère de Ste Catherine est un site du patrimoine } \\
\text { mondial. }\end{array}$ \\
\hline
\end{tabular}

Autant que possible, les gardiens des sites sacrés devraient participer à leur gestion. Les gardiens traditionnels des sites sacrés devraient communiquer et transmettre les valeurs culturelles et spirituelles des sites sacrés pour aider à déterminer les objectifs de la gestion. Les sites sacrés offrent une excellente occasion de s'engager dans ce dialogue et de développer des synergies qui sont environnementalement durables et socialement équitables.

\section{Sites sacrés et catégories d'aires protégées}

Quant à savoir si des sites naturels sacrés donnés doivent être inclus officiellement dans les systèmes nationaux d'aires protégées, cela dépend du souhait du groupe religieux et aussi du fait de savoir si les objectifs de gestion du site répondent à la définition d'une aire protégée par l'UICN et aux exigences d'une catégorie particulière. Ceci implique que le groupe religieux reconnaisse et accepte l'importance de la préservation de la biodiversité conjointement aux valeurs sacrées du site.

Il faut faire attention à ce que les valeurs culturelles et spirituelles ne mettent pas en danger celles de la biodiversité et que, inversement, la gestion d'une aire protégée n'endommage pas les valeurs sacrées du site. Intégrer efficacement les sites sacrés et, plus généralement, percevoir le caractère sacré de la nature dans les plans de conservation n'est possible que par une approche qui transcende les frontières idéologiques, physiques et institutionnelles. En bref, c'est un processus qui intègre la connaissance et la sagesse à la conservation de la biodiversité. C'est pourquoi, inclure les sites sacrés dans toutes les catégories d'aires protégées ajoute à leurs valeurs interculturelles et transversales qui, à leur tour, peuvent produire des synergies équitables entre la diversité spirituelle, culturelle et naturelle, en soutien à des objectifs de conservation plus holistiques.

\section{Géodiversité}

"La géodiversité est toute la variété de roches, de minéraux, de fossiles, de topographies, de sédiments et de sols, ainsi que les processus naturels qui les forment et les altèrent ».

De nombreuses aires protégées présentent une géodiversité importante, et certaines aires protégées sont créées en premier lieu pour leurs valeurs dans ce domaine; dans les deux cas, le maintien de ces valeurs exige une attention spéciale dans les politiques de gestion. La géodiversité est incluse dans les termes « conservation de la nature " de la définition par l'UICN d'une aire protégée.

La géodiversité constitue le fondement de la vie et de la diversité des habitats naturels et des paysages sur terre. De nombreux éléments géologiques et topographiques particuliers ont, pour les hommes, une valeur culturelle et emblématique qui influence la façon dont nous voyons les habitats naturels ou semi-naturels qui nous entourent. La géodiversité a aussi une profonde influence sur de nombreux aspects de paysages culturels, d'environnements bâtis et d'activités économiques. La protection de la géodiversité peut être une réponse à des intérêts divers, tels que ceux qui sont liés à des sites importants pour les fossiles, des sites de référence pour toutes les sciences de la terre, des formations spectaculaires auxquelles le tourisme est lié, et des modelés qui ont une valeur culturelle ou spirituelle particulière. La géodiversité peut contribuer au développement économique durable par un tourisme lié à des éléments géologiques. Comprendre les liens fonctionnels entre géodiversité 
et biodiversité est particulièrement important pour la gestion de la conservation dans des environnements dynamiques, où les processus naturels (p.ex. inondations, érosion et sédimentation) préservent la diversité de l'habitat et les fonctions écologiques. Ceci est explicite dans l'approche par écosystème et c'est fondamental à une époque où de nombreux écosystèmes sont confrontés aux impacts des changements climatiques. La géodiversité est donc une notion clé dans la gestion durable des terres, des rivières et des côtes. Elle exige des approches intégrées de la gestion du patrimoine naturel, de la terre et de l'eau à l'échelle du paysage/écosystème, qui sont basées sur la compréhension et l'expérience des processus naturels et de leurs réponses probables aux changements climatiques.

Bien que la catégorie III accorde une attention évidente à la protection d'éléments géologiques et de topographies spécifiques, la géodiversité peut être, et est, protégée dans toutes les catégories de l'UICN et par tous les types de gouvernance. Certains exemples sont donnés ci-dessous dans le Tableau 14.
Tableau 14. Exemples de géodiversité dans différentes catégories UICN d'aires protégées

\begin{tabular}{|l|l|l|}
\hline Catégorie & Exemple & Pays \\
\hline Ia & $\begin{array}{l}\text { Parc National du Grand } \\
\text { Canyon }\end{array}$ & Etats-Unis \\
\hline Ib & $\begin{array}{l}\text { Jenolan Karst Conservation } \\
\text { Reserve }\end{array}$ & Australie \\
\hline III & $\begin{array}{l}\text { Brecon Beacons National } \\
\text { Park }\end{array}$ & GB \\
\hline IV & & \\
\hline V & & \\
\hline VI &
\end{tabular}

Bien qu'il ne soit pas définitif, le Tableau 15 ci-dessous donne quelques indications pour savoir quand les valeurs de la géodiversité peuvent correspondre à des catégories de l'UICN données d'aires protégées.

Tableau 15. Indications des catégories UICN appropriées pour différents aspects de la géodiversité

\begin{tabular}{|l|l|}
\hline Aspect particulier de la géodiversité & Catégorie(s) appropriée(s) \\
\hline $\begin{array}{l}\text { La protection vise d'abord un élément d'intérêt particulier (monument } \\
\text { naturel tel qu'une chute ou une grotte) ou un site de valeur nationale } \\
\text { ou internationale pour les géosciences. }\end{array}$ & Principalement catégorie III \\
\hline $\begin{array}{l}\text { Un assemblage de topographies (p.ex. système terrestre de vallée } \\
\text { glaciaire) et/ou de processus, ou des éléments géologiques. }\end{array}$ & Principalement catégories la, Ib, II et V \\
\hline $\begin{array}{l}\text { Les éléments ont un potentiel d'interprétation et géotouristique. } \\
\text { La géodiversité est elle-même au fondement d'habitats et d'espèces } \\
\text { (p.ex. des plantes calcicoles ou des espèces adaptées aux grottes). }\end{array}$ & $\begin{array}{l}\text { Principalement catégories la, Ib, II, IV, V } \\
\text { et VI }\end{array}$ \\
\hline $\begin{array}{l}\text { La géodiversité a des liens importants avec les paysages culturels } \\
\text { (p.ex. des grottes utilisées comme habitations troglodytiques ou des } \\
\text { topographies adaptées à la culture en terrasses). }\end{array}$ & $\begin{array}{l}\text { Principalement catégorie V et aussi } \\
\text { catégories II et III }\end{array}$ \\
\hline $\begin{array}{l}\text { La géodiversité est la base du développement durable (activités } \\
\text { associées à des processus naturels, comme la visite de grottes). }\end{array}$ & $\begin{array}{l}\text { Principalement compatible avec les } \\
\text { catégories V et VI }\end{array}$ \\
\hline
\end{tabular}

\section{Restauration et catégories d'aires protégées de I'UICN}

La catégorie UICN d'aire protégée est d'abord choisie en fonction de l'objectif de gestion, c.-à-d. qu'elle est liée aux buts de la gestion plutôt qu'au statut, de sorte que toute catégorie peut faire l'objet d'une restauration. Cependant, en pratique, la catégorie implique aussi quelque chose au sujet du statut de l'aire protégée, et une restauration active n'est généralement pas souhaitable pour chaque catégorie d'aire protégée. Par exemple, la catégorisation en fonction des valeurs de nature sauvage (Ib) ne convient d'habitude pas pour une aire qui demande des interventions actives et continues de la gestion pour préserver ces valeurs. Dans certains cas, la restauration dans une aire protégée pourra être une intervention de durée limitée pour réparer des dommages passés alors que dans d'autres, les changements ont été si profonds qu'une intervention continue sera nécessaire à long terme : c'est souvent vrai lorsque certaines composantes écologiques, telles des espèces importantes, ont disparu. Certaines interventions, comme le contrôle d'espèces invasives et, dans certains habitats et à certaines conditions, des feux programmés, peuvent être nécessaires dans toute catégorie. Les conseils suivants décrivent la situation générale, mais il y a des exceptions :

- Restauration par des processus naturels suite à la protection (mise en défens) : par exemple, la restauration d'une forêt primaire en supprimant les abattages ou la pression du pâturage ; reconstitution des stocks de poissons ou de récifs de corail grâce à la limitation 
de la pêche ; suppression des risques de piétinement dans des communautés végétales de montagne - appropriée dans n'importe quelle catégorie d'aire protégée.

- Restauration par des interventions ponctuelles pour réparer des dommages passés : une ou plusieurs interventions pour réparer le dommage : par exemple, la réintroduction d'espèces disparues ; le repeuplement pour hâter la régénération d'une forêt ; la sélection des jeunes plants ; les éclaircies ; l'enlèvement des espèces invasives - généralement pas approprié dans les aires strictement protégées de catégorie $\mathrm{Ia}$ ou $\mathrm{Ib}$, mais bien dans les autres. ${ }^{15}$
- Restauration en tant que processus continu pour la préservation de la biodiversité : par exemple, le maintien artificiel du niveau de l'eau dans une zone humide d'un bassin qui a subi des changements hydrologiques majeurs ; l'émondage des taillis pour préserver une forêt culturelle importante ; le recours au pâturage du bétail domestique pour conserver des valeurs de la biodiversité généralement appropriée pour les catégories IV à VI.

- Restauration en tant que processus continu, tant pour les ressources naturelles que pour la biodiversité : par exemple, recouvrer une productivité après l'érosion du sol, fournir des ressources pour le bien-être des hommes appropriée pour les catégories V-VI.

Tableau 16. Guide indicatif pour la restauration dans différentes catégories UICN

\begin{tabular}{|c|c|c|c|c|c|c|}
\hline \multicolumn{7}{|c|}{ Catégorie UICN } \\
\hline la & lb & II & III & IV & $\mathbf{V}$ & VI \\
\hline \multicolumn{7}{|c|}{ Restauration par des processus naturels suite à la protection } \\
\hline & & \multicolumn{5}{|c|}{ Restauration active et ponctuelle } \\
\hline & & & & \multicolumn{3}{|c|}{ Restauration continue pour la biodiversité } \\
\hline & & & & & \multicolumn{2}{|c|}{$\begin{array}{l}\text { Restauration continue pour la } \\
\text { biodiversité et les besoins humains }\end{array}$} \\
\hline
\end{tabular}

Dans le cas où la destruction générale de l'habitat a progressé si fort que les aires protégées elles-mêmes exigent une restauration substantielle, il peut être indiqué d'attendre et de voir comment les projets de restauration marchent avant d'assigner une catégorie. Le degré de restauration et la gestion active requis peuvent augmenter dans de nombreuses aires protégées en raison des changements climatiques.

15 Il est possible pour une aire protégée d'être reclassée sous la catégorie Ia ou Ib si la restauration a porté ses fruits. 



\section{Initiatives internationales de conservation}

II existe un certain nombre de tentatives parallèles pour protéger des habitats clés relevant d'accords des Nations unies ou régionaux. La Convention sur la diversité biologique, les sites du patrimoine mondial de l'UNESCO, les réserves de biosphère de l'UNESCO et les sites Ramsar sont particulièrement pertinents. La section suivante examine en particulier comment Ramsar et le Patrimoine mondial sont liés aux catégories de l'UICN. 


\section{Convention du patrimoine mondial}

Les sites du patrimoine mondial comptent parmi les endroits culturels et naturels les plus importants du monde; ils sont reconnus par la Convention du patrimoine mondial de l'UNESCO et jouissent d'une protection particulière de la part de leur pays-hôte. Ils incluent des monuments comme Angkor Wat au Cambodge ou les pyramides d'Egypte, ainsi que des aires naturelles exceptionnelles comme le Parc National de Serengeti, en Tanzanie, ou le Parc National de Canaima, au Venezuela. Les gouvernements proposent des sites pour une possible inclusion sur la Liste du patrimoine mondial, et leur reconnaissance dépend d'une évaluation technique, ${ }^{16}$ suivie d'une revue et de la décision finale prise par les membres du Comité du patrimoine mondial. La pertinence se fonde en partie sur la question de savoir si le site est d'une valeur universelle exceptionnelle, termes qui font référence à la combinaison des valeurs patrimoniales d'un site qui montrent pourquoi il a une valeur mondiale et aussi qu'il respecte les exigences en matière d'intégrité et de gestion efficace. L'UICN est officiellement reconnu dans le texte de la Convention comme un organisme consultatif pour tous les sites naturels ou mixtes (naturels et culturels). Ceci implique de réaliser des évaluations techniques de tous les sites candidats et de mener des missions de suivi comme il est exigé pour des sites existants qui sont menacés. Pratiquement tous les sites naturels du patrimoine mondial sont aussi des aires protégées. Dans le passé, les sites du patrimoine mondial étaient inscrits séparément sur la Liste des Nations unies des aires protégées, mais cela a entraîné des répétitions parce que beaucoup étaient aussi listés selon leur catégorie UICN.

\section{Qu'est-ce que la Convention du patrimoine mondial exige des sites naturels de la Liste du patrimoine mondial ?}

Les notes suivantes visent à aider les gouvernements à envisager la relation entre les sites naturels du patrimoine mondial et le système des catégories d'aires protégées de l'UICN. Elles ne couvrent pas les sites culturels qui, pour la plupart, ne se trouvent pas dans des aires protégées (ou, s'ils s'y trouvent, c'est seulement par hasard).

\section{La relation entre patrimoine mondial et aires protégées, en théorie}

La version 2008 des Orientations devant guider la mise en ouvre (OG) de la Convention du patrimoine mondial explique ce qui est requis dans le cadre du patrimoine mondial (PM). Il y est dit qu'une aire ne peut être inscrite sur la Liste du PM que si le site répond aux critères pertinents du PM et s'il remplit les conditions strictes d'intégrité et de conservation (paragraphe 88), ce qui signifie qu'il doit :
- Inclure tous les éléments nécessaires pour exprimer la Valeur universelle exceptionnelle pour laquelle il a été nominé pour être inscrit sur la liste du PM ;

- Etre d'une taille adéquate pour garantir la représentation complète des caractéristiques et des processus qui conferent au site toute sa signification ;

- Ne pas souffrir d'effets négatifs du développement ni/ou de la négligence.

Les sites PM potentiels sont jugés par rapport à plusieurs critères dont deux (écosystèmes et biodiversité) sont particulièrement pertinents pour des aires protégées. Les paragraphes 94-95 décrivent les exigences d'intégrité pour ces deux critères :

- Critère ix (écosystèmes) : Les sites « doivent être assez étendus et contenir les éléments nécessaires à l'illustration des principaux aspects des processus essentiels à la conservation à long terme des écosystèmes et de la diversité biologique qu'ils contiennent ".

- Critère x (biodiversité) : Les sites " doivent contenir des habitats pour le maintien d'un maximum de diversité animale et végétale caractéristique des provinces et écosystèmes biogéographiques concernés ».

Les OG reconnaissent qu' " aucune zone n'est totalement intacte et que toutes les aires naturelles sont dans un état dynamique et, dans une certaine mesure, entrainent des contacts avec des personnes. Il y a souvent des activités humaines, dont celles de sociétés traditionnelles et de communautés locales, dans des aires naturelles. Ces activités peuvent être en harmonie avec la valeur universelle exceptionnelle de l'aire là où elles sont écologiquement durables» (para. 90).

Enfin, elles incluent une section intitulée Protection et gestion (para. 96-118), qui détaille les mesures requises pour la conservation à long terme d'aires nominées soumises au titre de PM. Spécifiquement, le paragraphe 97 déclare que : «Tous les biens inscrits sur la Liste du patrimoine mondial doivent avoir une protection législative, à caractère réglementaire, institutionnelle etlou traditionnelle adéquate à long terme pour assurer leur sauvegarde. Cette protection devra inclure des limites correctement défnies». Le paragraphe 98 ajoute encore que : «Des mesures législatives et à caractère réglementaire au niveau national et local assurent la survie du bien et sa protection contre un développement et des changements qui pourraient avoir un impact négatif sur la valeur universelle exceptionnelle ou l'intégrité(...) du bien. Les Etats parties doivent assurer la mise en cuvre totale et effective de ces mesures".

Quant à la relation entre les sites nominés et les aires protégées existantes, les OG stipulent, au paragraphe 102

16 Tous les sites naturels sont évalués par l'UICN et les sites culturels par ICOMOS - le Conseil international des monuments et des sites. 
que : "Les limites du bien proposé pour inscription peuvent coïncider avec une ou plusieurs aires protégées existantes ou proposées, telles que des parcs nationaux, des réserves naturelles, des réserves de biosphère [....]. Alors que ces aires créées dans un but de protection peuvent contenir plusieurs zones de gestion, seules certaines de ces zones peuvent répondre aux critères d'inscription". Cette déclaration implique que certaines aires ayant une protection légale peuvent ne pas encore se qualifier pour le statut de PM, c.-à-d. que certaines formes de protection légale ne sont pas assez restrictives pour satisfaire aux exigences des OG.

Donc, même si les OG ne disent pas qu'un site doit être une " aire protégée » et ne se réferent aux catégories d'aires protégées de l'UICN, on pourrait en déduire que des aires qui ne bénéfcient d'aucun régime spécial de protection devraient être exclues des sites du PM (p.ex. OG paragraphes 97 et 102); dès lors, on peut s'attendre à ce que les sites du patrimoine mondial soient gérés d'une façon équivalente aux aires protégées, qu'ils soient ou non formellement protégés. C'est l'interprétation appliquée par l'UICN dans son rôle consultatif.

\section{La relation entre sites du patrimoine mondial et aires protégées, en pratique}

Avoir un régime de gestion efficace est une des exigences pour figurer sur la Liste du patrimoine mondial et, en pratique, cela signifie que la grande majorité des sites naturels du patrimoine mondial sont des aires protégées. Le WCMC/PNUE prépare des fiches de données pour tous les sites proposés au patrimoine mondial, et celles-ci indiquent explicitement la catégorie d'aire protégée de l'UICN dans laquelle le site proposé est classé. Il existe donc un lien évident entre les sites du patrimoine mondial et le système de catégories.
Cette situation a évolué graduellement. Au cours des premières années de la Convention, certains sites naturels du patrimoine mondial incluaient des développements qui, aujourd'hui, ne seraient plus acceptés par le Comité du patrimoine mondial. Par conséquent, certains sites du patrimoine mondial englobent des aires où l'utilisation est incompatible avec le statut du patrimoine mondial et qui sont assez vastes pour être considérées comme des aires clairement définies au sein d'un site du PM, et pas simplement comme des intrusions mineures "préexistantes " dans une aire protégée, par ailleurs relativement intacte. Les Etats parties peuvent, en théorie, proposer des amendements pour retrancher certaines aires de leurs anciennes nominations. Cela arrive dans certains cas, mais cela exige un examen attentif au cas par cas. ${ }^{17}$

La plupart des sites du PM existants ou nominés correspondent aux limites d'aires protégées existantes. Lorsque de grands espaces séparent des aires protégées qui ont des valeurs semblables ou complémentaires, il est possible d'inscrire une nomination en série, et ce genre de nomination est de plus en plus courant (p.ex. la Côte de la Découverte - Réserves de la forêt atlantique du Brésil et la Région florale du Cap d'Afrique du Sud). Les études de cas présentées au Tableau 17 montrent comment ce durcissement s'est réalisé au cours des 25 dernières années. De nouveaux sites du PM se sont peu à peu conformés plus strictement à la définition de l'UICN d'une aire protégée, et des aires qui ne bénéficiaient pas d'un régime de protection ont été de plus en plus exclues. Cependant, il reste des exceptions (p.ex. la presqu'île de Valdés, en Argentine), et ce n'est pas encore une condition pour un site naturel du PM d'être une aire protégée officielle si une protection et une gestion adéquates peuvent être assurées par d'autres moyens.

Tableau 17. Evolution de la relation entre sites naturels du patrimoine mondial et aires protégées

\begin{tabular}{|c|c|c|c|c|}
\hline Nom du site & $\begin{array}{l}\text { Critères } \\
\text { PM }\end{array}$ & $\begin{array}{l}\text { Cat. } \\
\text { UICN }\end{array}$ & $\begin{array}{l}\text { Année } \\
\text { inscr. }\end{array}$ & Discussion \\
\hline $\begin{array}{l}\text { lles Galápagos } \\
\text { EQUATEUR }\end{array}$ & $\begin{array}{l}\text { vii, viii, } \\
\text { ix, } x\end{array}$ & $\begin{array}{l}\text { Il (terre) } \\
\text { IV } \\
\text { (marine) }\end{array}$ & 1978 & $\begin{array}{l}\text { Parmi le premier lot de nominations jamais soumis pour inscription } \\
\text { sur la Liste du PM, les limites terrestres n'excluent pas les } \\
\text { surfaces agricoles ni les installations, ce qui résulte en un site qui } \\
\text { comprend des ranches étendus et des zones urbaines densément } \\
\text { peuplées. Le site fut étendu en } 2001 \text { pour inclure une aire marine } \\
\text { protégée qui contient un mélange de zones à usage multiple de } \\
\text { faible intensité (plongée, pêche artisanale). }\end{array}$ \\
\hline $\begin{array}{l}\text { Grande Barrière } \\
\text { de Corail } \\
\text { AUSTRALIE }\end{array}$ & $\begin{array}{l}\text { vii, viii, } \\
\text { ix, } x\end{array}$ & $\mathrm{~V}$ & 1981 & $\begin{array}{l}\text { Une zone à usage multiple, avec une variété d'utilisations } \\
\text { autorisées, allant de la conservation stricte aux loisirs, } \\
\text { y compris la pêche. Dans le rapport d'évaluation de sa } \\
\text { nomination, l'UICN a suggéré que les limites actuelles du site } \\
\text { du PM soient ramenées à celles de la zone noyau totalement } \\
\text { protégée (ces commentaires ne se trouvaient pas dans } \\
\text { l'évaluation de la nomination des Galápagos), mais elle a fini } \\
\text { par recommander, dans le même rapport, que la nomination } \\
\text { soit inscrite sous la forme proposée à l'origine. }\end{array}$ \\
\hline
\end{tabular}

17 Par exemple, l'exclusion du domaine skiable du site du PM est actuellement à l'étude dans le Parc National de Pirin, en Bulgarie. 
Tableau 17. Evolution de la relation entre sites naturels du patrimoine mondial et aires protégées (suite)

\begin{tabular}{|c|c|c|c|c|}
\hline Nom du site & $\begin{array}{l}\text { Critères } \\
\text { PM }\end{array}$ & $\begin{array}{l}\text { Cat. } \\
\text { UICN }\end{array}$ & $\begin{array}{l}\text { Année } \\
\text { inscr. }\end{array}$ & Discussion \\
\hline $\begin{array}{l}\text { Lac Baikal } \\
\text { FEDERATION } \\
\text { DE RUSSIE }\end{array}$ & $\begin{array}{l}\text { vii, viii, } \\
\text { ix, } x\end{array}$ & la, II, IV & 1996 & $\begin{array}{l}\text { Le site se compose de différentes entités de gestion de la } \\
\text { conservation ainsi que de terres dépourvues de mesures } \\
\text { de conservation (p.ex. zones de protection côtières), d'une } \\
\text { valeur limitée pour la conservation. On y trouve une gamme } \\
\text { d'utilisations potentiellement incompatibles, y compris la pêche } \\
\text { commerciale, les coupes de bois, l'agriculture, la chasse et } \\
\text { le tourisme. Il y a aussi plusieurs petites installations dans le } \\
\text { site. Les recommandations originales pour la limite du site } \\
\text { du PM avaient inclus une superficie beaucoup plus grande, } \\
\text { comprenant des villes importantes, mais ce fut finalement une } \\
\text { surface plus réduite, avec moins d'utilisations conflictuelles, qui } \\
\text { fut inscrite. }\end{array}$ \\
\hline $\begin{array}{l}\text { East Rennell } \\
\text { ILES } \\
\text { SALOMON }\end{array}$ & ix & $\mathrm{n} / \mathrm{a}$ & 1998 & $\begin{array}{l}\text { Près de } 800 \text { personnes d'origine polynésienne résident dans } \\
\text { le site. Elles y pratiquent l'agriculture de subsistance, la } \\
\text { pêche et la chasse. La population locale dépend des produits } \\
\text { de la forêt pour la plupart des matériaux de construction. } \\
\text { Le régime foncier est coutumier, et un lac d'eau douce est } \\
\text { considéré comme un bien commun. Ce fut le premier site } \\
\text { naturel du patrimoine mondial à être inscrit sur la Liste du } \\
\text { patrimoine mondial sous un régime de gestion coutumier. } \\
\text { Dans ce cas, le Comité du PM, sur la recommandation de } \\
\text { l'UICN, a remarqué que le régime de gestion coutumier était } \\
\text { suffisamment efficace pour garantir la protection des valeurs } \\
\text { naturelles. }\end{array}$ \\
\hline $\begin{array}{l}\text { Presqu'île de } \\
\text { Valdés } \\
\text { ARGENTINE }\end{array}$ & $x$ & II, IV, VI & 1999 & $\begin{array}{l}\text { Un ensemble de sept aires protégées distinctes et d'une } \\
\text { proportion significative (>50 pour cent) de terres privées. } \\
\text { Les propriétaires sont encouragés à collaborer par un } \\
\text { exercice conjoint de planification de la gestion, mais ils ne } \\
\text { semblent pas être tenus par la loi de le faire. Les menaces } \\
\text { actuelles incluent la subdivision des terres en vue d'un } \\
\text { développement résidentiel de la côte. Ceci semble être une } \\
\text { expérience dans des propriétés privées au sein d'un site } \\
\text { naturel du PM. }\end{array}$ \\
\hline $\begin{array}{l}\text { Côte de la } \\
\text { Découverte- } \\
\text { Réserves de la } \\
\text { forêt atlantique } \\
\text { BRESIL }\end{array}$ & ix, $x$ & la, II & 1999 & $\begin{array}{l}\text { Un ensemble de huit aires protégées distinctes dispersées sur } \\
\text { plus de } 450 \mathrm{~km}^{2} \text { et nichées dans une réserve de biosphère } \\
\text { d'un million d'ha - les terrains interstitiels sont en grande partie } \\
\text { privés. }\end{array}$ \\
\hline $\begin{array}{l}\text { Aires protégées } \\
\text { de la Région } \\
\text { florale du Cap } \\
\text { AFRIQUE DU } \\
\text { SUD }\end{array}$ & ix, $x$ & Ib, II, IV & 2004 & $\begin{array}{l}\text { L'inscription de ce site sériel est le résultat d'un processus de } \\
\text { plusieurs années au cours duquel la nomination originale de } \\
\text { l'Etat partie ne fut pas acceptée en raison de l'absence de } \\
\text { régime de gestion consolidé pour l'ensemble des sept aires } \\
\text { protégées. Ensuite, une nomination finale fut soumise, qui } \\
\text { remplissait les conditions techniques imposées par l'UICN, et } \\
\text { fut inscrite par le Comité du PM. }\end{array}$ \\
\hline $\begin{array}{l}\text { Sanctuaires du } \\
\text { grand panda du } \\
\text { Sichuan } \\
\text { CHINE }\end{array}$ & $x$ & $\mathrm{n} / \mathrm{a}$ & 2006 & $\begin{array}{l}\text { Les limites originales proposées par l'Etat partie incluaient } \\
\text { des villes, des zones agricoles et des travaux d'infrastructures } \\
\text { publics. Les examens de la nomination originale durèrent plus } \\
\text { de dix ans. L'UICN a demandé de revoir les limites de sorte } \\
\text { que seules les aires centrales protégées soient incluses. Les } \\
\text { limites finales reflètent la demande de l'UICN. }\end{array}$ \\
\hline
\end{tabular}




\section{La relation entre sites du patrimoine mondial et catégories d'aires protégées de I'UICN}

Si tous les sites naturels du patrimoine mondial ne sont pas des aires protégées, il s'ensuit que tous n'auront pas de catégorie UICN. Mais en pratique, la plupart sont des aires protégées et la plupart relèvent vraiment de catégories. Il y a des sites naturels du patrimoine mondial dans toutes les catégories de l'UICN, mais avec un biais certain en faveur des objectifs de gestion plus stricts des catégories Ia, Ib et II. En juin 2008, il y avait 166 biens naturels et 25 biens mixtes au patrimoine mondial. De ceux-ci, 139 sont inscrits sous les critères ix et/ou x (et donc centrés sur des questions de biodiversité et d'espèces), soit exclusivement, soit en combinaison avec les critères " non-biodiversité " vii et viii, et ils sont considérés comme des sites naturels du patrimoine reconnus pour leur biodiversité. La Figure 4 illustre la fréquence d'occurrence d'une catégorie donnée d'aire protégée de l'UICN dans les sites naturels du PM. ${ }^{18}$

Figure 4. Fréquence de l'occurrence des catégories d'aires protégées de l'UICN dans les sites naturels de biodiversité et de non-biodiversité du PM

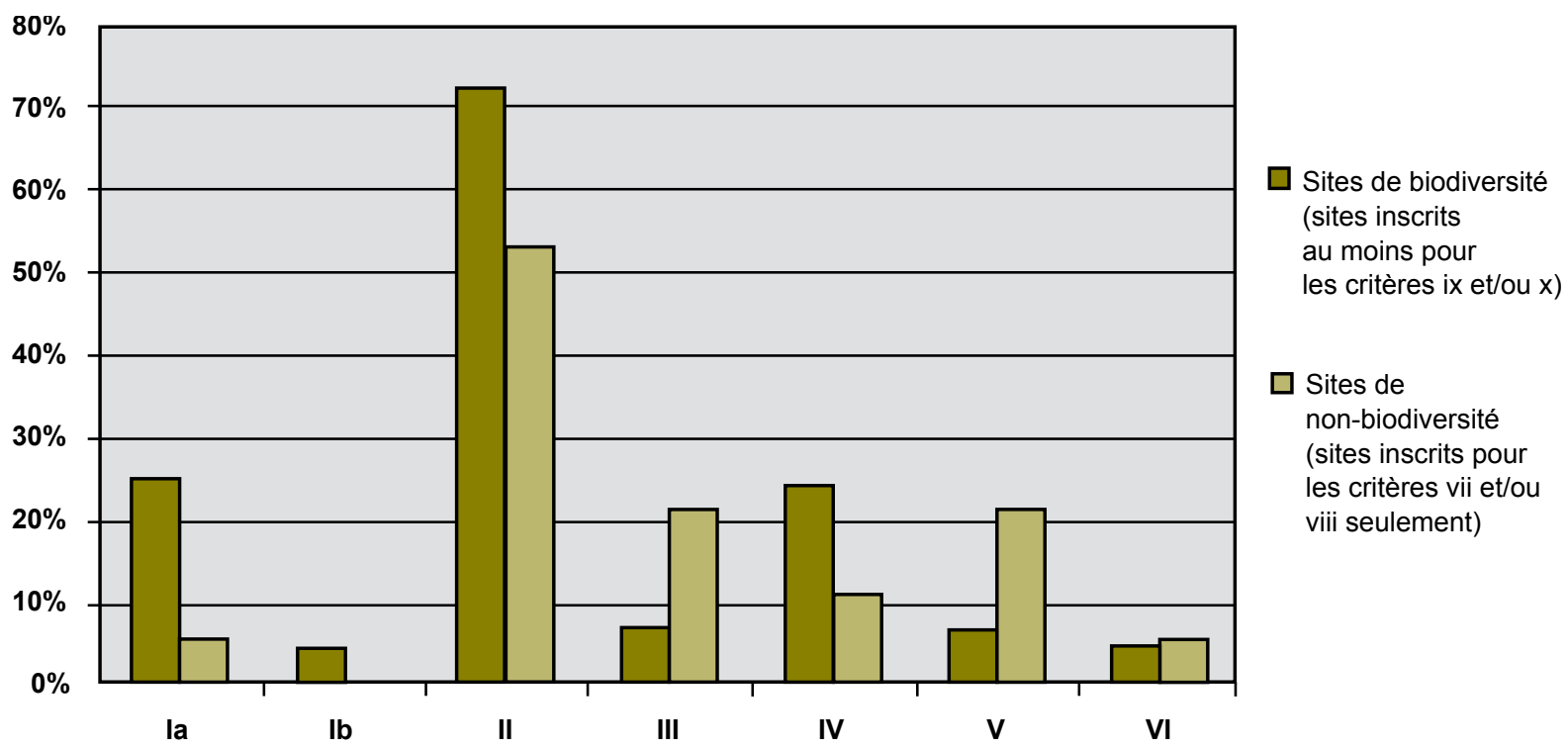

Plus de 70 pour cent des sites du patrimoine mondial listés pour les valeurs de biodiversité contiennent (en tout ou en partie) une aire protégée de catégorie II. Certains de ces mêmes sites contiennent aussi des aires protégées d'autres catégories (par exemple, le Te Wahipounamu, en Nouvelle-Zélande, se compose de plusieurs aires protégées différentes représentant cinq catégories différentes d'aires protégées). Le diagramme montre que peu de sites de biodiversité du PM contiennent des aires protégées de catégorie V et VI (ces catégories sont représentées dans huit et six sites de PM respectivement, sur les 128 sites auxquels la base de données du WCMC/PNUE attribue une catégorie d'aire protégée). De ceux-ci, seuls trois (2 pour cent de tous les sites de biodiversité) se composent exclusivement d'une aire protégée de catégorie V ou VI - la Grande Barrière de corail, en Australie (qui est en train de changer), le Parc National du Banc d'Arguin, en Mauritanie (considéré d'habitude comme de catégorie II) et la Zone de conservation de Ngorongoro, en Tanzanie. Ce sont généralement de grands sites : $348.700 \mathrm{~km}^{2}, 12.000 \mathrm{~km}^{2}$ et $8.288 \mathrm{~km}^{2}$ respectivement.

\section{Conclusions}

Pour les gouvernements qui envisagent de proposer la nomination d'un site naturel au patrimoine mondial :

- Tous les sites naturels du patrimoine mondial doivent avoir un régime de gestion efficace. Cela implique que ces aires seront désignées comme aires protégées dans pratiquement tous les cas.

- Il n'existe pas de règle pour décréter que ces sites doivent être assignés à une catégorie de l'UICN mais, de nouveau, ceci est fortement encouragé et, en fait, toutes les fiches de données du WCMC pour les sites naturels proposés au PM indiquent une (des) catégorie(s) de l'UICN qui correspond(ent) au site proposé. La plupart des sites inscrits pour les critères ix ou x correspondent aux catégories I ou II de l'UICN ; cependant, il y a des exceptions et toute catégorie peut être acceptable.

18 Etant donné qu'un site du PM peut être composé de plusieurs aires protégées, auxquelles sont assignées différentes catégories, le nombre total ne correspond pas à 100 pour cent. D'autre part, seuls 128 des 139 sites de biodiversité, et 38 des 47 sites de non-biodiversité se sont vu attribuer une catégorie d'aire protégée dans la BDMAP. 


\section{Convention de Ramsar}

La Convention de Ramsar encourage les Parties à créer et à gérer d'importantes zones humides d'une manière qui ne modifie pas leur caractère écologique. Les 158 Parties contractantes (gouvernements) se sont engagées à l'« utilisation rationnelle » de toutes les zones humides de leur territoire (y compris les rivières), à la conservation des "zones humides d'importance internationale " (sites Ramsar) et à la coopération internationale. Les Parties s'engagent chacune à entreprendre un inventaire de leurs zones humides et à préparer un " cadre stratégique pour la liste Ramsar " pour la désignation nationale systématique et représentative et pour la gestion de types d'habitats en zone humide. La Convention présente beaucoup d'avantages pour la conservation des zones humides parce qu'elle crée une pression morale sur les gouvernements membres afin qu'ils établissent et gèrent des aires protégées en zones humides ; elle instaure des normes, fournit des orientations et facilite la collaboration pour l'utilisation rationnelle; elle a un système mondial triennal d'établissement de rapports et de suivi ; et elle encourage la participation d'ONG, de communautés locales et de populations autochtones.

Alors que de nombreuses zones humides d'importance internationale (sites Ramsar) ont aussi un statut de protection (p.ex. sont des aires protégées reconnues par la législation nationale, des sites du patrimoine mondial ou des réserves de biosphère de l'UNESCO), il n'y a aucune obligation que les sites Ramsar soient des aires légalement protégées par une législation nationale. En fait, cela aide parfois à persuader les gouvernements à désigner des sites relevant de la Convention de Ramsar alors qu' ils seraient réticents à l'idée d'en faire des aires protégées.

La protection qu'offre la Convention est en soi un support légal, mais selon un droit non contraignant et pas toujours aussi clairement articulé. Par exemple, les Critères pour l'identification des zones humides d'importance internationales ne font pas référence au statut de protection. La Fiche d'information sur les zones humides de Ramsar implique que le statut de protection n'est pas obligatoire, avec des phrases comme : "Si une réserve a été créée ". Le Manuel de la Convention de Ramsar (2006) est explicite : "Désigner une zone humide pour la liste de Ramsar ne requiert pas, en soi, que le site ait été au préalable déclaré aire protégée ». En fait, l'inscription sur la liste de la Convention de Ramsar, spécialement dans le cas de sites qui font l'objet d'une utilisation intense par les communautés humaines - que ce soit pour extraire des ressources ou pour profiter des fonctions naturelles de la zone humide - peut apporter la protection nécessaire pour assurer sa durabilité à long terme. Ceci peut se faire au mieux en préparant et en réalisant un plan de gestion approprié, avec la participation active de toutes les parties prenantes.

Comme l'implique ce qui précède, inscrire un site sur la liste de Ramsar, spécialement dans le cas de sites qui font l'objet d'une utilisation intense par des communautés humaines, devrait offrir la protection nécessaire pour garantir sa durabilité à long terme. L'inscription Ramsar élève les sites à un statut supérieur, attire davantage l'attention sur eux et devrait contribuer à leur conservation à long terme et à leur utilisation rationnelle - que le statut Ramsar leur confere, ou pas, une protection légale supplémentaire dans le pays dépend des décisions des gouvernements national et local. Les utilisations des zones humides par les hommes sont compatibles avec le statut Ramsar, pour autant qu'elles répondent au concept de Ramsar d' " utilisation rationnelle " (durable) et n'entraînent pas un changement négatif de leur caractère écologique.

Le Secrétariat de la Convention de Ramsar considère parfois la liste de Ramsar comme un ensemble d' " aires protégées »: par exemple, le document Les solutions d'urgence conduisent rarement à la durabilité donne " une introduction au concept de Zones humides d'importance internationale comme un réseau d'aires protégées (c'est nous qui soulignons). Certaines Parties regardent l'inclusion dans la liste comme signifiant, de fait, que le site devient une aire protégée (qu'il ait, ou pas, une catégorie UICN), alors que d'autres pas.

\section{Le système des catégories et les sites Ramsar}

Les versions originales des catégories de gestion, des réserves de biosphère et des sites du patrimoine mondial furent identifiées comme des catégories indépendantes, mais les sites Ramsar n'étaient pas identifiés comme tels. Par la suite, les lignes directrices de 1994 n'ont traité aucune désignation internationale comme une catégorie séparée. Lors de la neuvième Conférence des Parties à la Convention de Ramsar (Résolution IX.22), il fut accepté d'inclure des données au sujet des catégories de l'UICN dans la base de données des sites Ramsar. Sur les 84 sites désignés depuis le $1^{\text {er }}$ janvier 2007, 37 (44 pour cent) incluent des informations sur la catégorie de l'UICN. Les sites Ramsar sont désignés au niveau national. Le système des catégories de l'UICN est un moyen de les classer sur la base de leurs objectifs de gestion. Les sites Ramsar " collent " très exactement à cette approche parce que le concept lui-même incarne l'idée d'un éventail d'objectifs de gestion. D'autre part, certains sites Ramsar englobent souvent une série de zones qui ont des objectifs de gestion différents, qui peuvent chacun correspondre à une catégorie du système de l'UICN. Certains peuvent se composer d'un certain nombre de catégories d'utilisations différentes.

Les lignes directrices de l'UICN proposent plusieurs moyens de réconcilier les nombreuses situations différentes susceptibles de se trouver dans des sites Ramsar avec le système des catégories. Une fois que l'on a déterminé que le site répond bien à la définition UICN d'une aire protégée, nous recommandons une approche en deux étapes :

- Etape 1. Identifier si l'ensemble du site Ramsar doit être classé dans une ou plusieurs catégories.

Pour ce faire, il est nécessaire d'établir laquelle de trois possibilités théoriques s'applique : 
1. Il n'y a qu'une seule autorité de gestion pour tout le site Ramsar et, à des fins juridiques, tout le site Ramsar est catégorisé par la loi comme ayant un objectif de gestion principal.

L'aire serait assignée à une seule catégorie.

Si les lignes directrices exigent que l'assignation soit fondée sur le principal objectif de gestion, elles reconnaissent aussi que les plans de gestion contiennent souvent des zones de gestion pour toute une variété de motifs, afin de tenir compte des circonstances locales. Afin d'établir quelle est la catégorie appropriée, au moins trois-quarts, et de préférence davantage, doivent être gérés en vue de l'objectif principal ; et la gestion du reste de la superficie ne doit pas être en conflit avec cet objectif principal.

2. Il y a une autorité de gestion responsable de deux ou trois aires constituant le site Ramsar, mais chacune de ces aires a des objectifs de gestion séparés, légalement définis par des objectifs de gestion.

Les lignes directrices tiennent compte de cette situation en reconnaissant que "des aires protégées de catégories différentes sont souvent contiguës, et parfois une catégorie est "nichée» au sein d'une autre ". C'est ainsi que de nombreuses aires de catégorie $\mathrm{V}$ englobent des aires de catégorie I et IV ; certaines seront adjacentes à des aires de catégorie II. Et certaines aires de catégorie II contiennent des aires de catégorie Ia et Ib.

Dans ce cas, les différentes parties du site Ramsar seront classées dans des catégories différentes.

3. Il y a deux ou plusieurs autorités de gestion responsables d'aires séparées, avec des objectifs de gestion différents, qui composent ensemble le site Ramsar.

Ici aussi, l'interprétation correcte des lignes directrices serait de classer ces aires séparément.

- Etape 2. Assigner des parties du site Ramsar à des catégories différentes.

Le système des catégories peut s'appliquer à toute une gamme de situations légales et managériales différentes qui caractérisent les sites Ramsar dans différents pays. Ceci est tout à fait en accord avec la manière dont le système est censé être appliqué. L'UICN déclare que des aires protégées doivent d'abord être établies pour répondre à des objectifs compatibles avec les buts et les besoins (ou un mélange de ceux-ci) nationaux, locaux ou privés, et seulement alors labellisées avec une catégorie de l'UICN qui correspond aux objectifs de leur gestion. Ces catégories ont été mises au point pour faciliter la communication et l'information, pas pour régenter le système.

\section{Avantages}

Les bénéfices d'un système qui peut s'appliquer au niveau international, de façon transparente, sont significatifs. Le principal avantage est qu'il permet des évaluations globales des sites Ramsar existants. De plus, il facilite le développement et l'établissement d'un système de sites Ramsar dans lequel chaque pays peut préserver son propre réseau de sites Ramsar, tout en faisant clairement partie du cadre global des aires protégées. Il permet aussi au réseau de sites Ramsar de se lier et de contribuer au développement d'un système mondial d'aires protégées, complet, approprié et représentatif.

Il est prévu de produire des orientations plus détaillées sur les liens entre les sites Ramsar et les catégories d'aires protégées de l'UICN.

\section{Convention sur la diversité biologique}

Lors de la septième réunion de la Conférence des Parties (COP7) à la CDB en 2004, 188 Parties ont accepté un Programme de travail sur les aires protégées, l'une des stratégies environnementales les plus ambitieuses de l'histoire. Le Programme vise d'ici 2010 (sur terre) et 2012 (en mer) à établir " des systèmes nationaux et régionaux d'aires protégées complets, efficacement gérés et écologiquement représentatifs ". Il compte plus de 90 actions cibles spécifiques et limitées dans le temps à destination des Etats membres et d'autres.

Spécifiquement, le Programme "reconnaît l'intérêt d'un système unique et international de classification des aires protégées et l'avantage de fournir des informations qui soient comparables entre les pays et les régions et, à cet égard, il se félicite des efforts de la CMAP/UICN visant à affiner le système de catégories de l'UICN et encourage les Parties, les autres gouvernements et les organisations compétentes à assigner des catégories de gestion à leurs aires protégées, en fournissant des informations conformes aux catégories affinées de l'UICN pour la présentation de rapports".

La CDB a donné sa propre définition d'une aire protégée comme : une aire définie géographiquement qui est désignée ou réglementée et gérée pour atteindre des objectifs de conservation spécifiques. Il existe un accord tacite entre le Secrétariat de la $\mathrm{CDB}$ et l'UICN pour reconnaître que les deux définitions signifient en fait la même chose. Le fait que le Programme de travail de la CDB reconnaît explicitement les catégories d'aires protégées de l'UICN est significatif :

Explorer l'établissement d'un système harmonisé et d'un calendrier pour l'établissement de rapports sur les sites désignés au titre de la Convention sur les zones humides, de la Convention sur le patrimoine mondial, du Programme sur L'homme et la biosphère de l'UNESCO, et d'autres systèmes régionaux, selon le cas, en tenant compte des travaux permanents du WCMC/PNUE sur l'harmonisation des rapports et le système de catégories de gestion des aires protégées de I'UICN aux fins d'établissement des rapports (c'est nous qui soulignons). 
Lors de la neuvième Conférence des Parties à la CDB, en 2008, le soutien aux catégories fut réaffirmé et confirmé :

"9. Réaffirme le paragraphe 31 de la décision VII/28 qui reconnaît l'intérêt d'un système unique et international de classification des aires protégées et l'avantage de fournir des informations qui soient comparables entre les pays et les régions et, à cet égard, il se félicite des efforts de la Commission mondiale des aires protégées de l'UICN visant à affiner le système de catégories de l'UICN et encourage les Parties, les autres gouvernements et les organisations compétentes à assigner des catégories de gestion à leurs aires protégées, en fournissant des informations conformes aux catégories affinées de l'UICN pour la présentation de rapports".

Il y a donc une orientation claire de la CDB pour que les pays utilisent le système des catégories de l'UICN pour rapporter les progrès réalisés dans l'établissement et la préservation de systèmes d'aires protégées. 


\section{Efficacité des catégories de l'UICN}

L'UICN a toujours insisté sur le fait qu'une catégorie est toujours fondée sur un objectif et indépendante de l'efficacité : si une aire protégée ne réussit pas à atteindre son objectif, ce n'est pas une excuse pour changer de catégorie (mais plutôt pour augmenter la capacité de gestion). Cependant, de nombreuses parties prenantes demandent une relation plus étroite entre catégorie et efficacité : la section suivante explore quelques options. 


\section{Evaluation de la gestion et les catégories de I'UICN}

Lefficacité de gestion des aires protégées reçoit de plus en plus d'attention en tant qu'élément essentiel pour le maintien d'un système d'aires protégées qui fonctionne bien, et l'évaluation ou l'appréciation de la gestion est considérée aujourd'hui comme un instrument très utile pour en augmenter l'efficacité, en fournissant aux gestionnaires, entre autres, des informations concises et pratiques. L'évaluation de l'efficacité de la gestion se définit comme l'appréciation de la façon dont les aires protégées sont gérées - et d'abord de la mesure dans laquelle elles protègent leurs valeurs et atteignent leurs buts et objectifs. Les termes « efficacité de gestion » reflètent trois « thèmes » majeurs dans la gestion des aires protégées :

- les questions de conception liées aussi bien aux sites particuliers qu'aux systèmes d'aires protégées;

- la pertinence et la justesse des systèmes et des processus de gestion ;

- l'atteinte des objectifs de l'aire protégée, y compris la conservation de ses valeurs.

L'évaluation est maintenant reconnue comme une composante d'une gestion responsable et proactive d'une aire protégée, aussi bien pour aider les gestionnaires à prendre les décisions journalières concernant l'allocation du temps et des ressources que (et de plus en plus) comme une étape nécessaire pour préparer des rapports sur les progrès de la conservation dans un contexte international, dans le cadre de conventions et d'accords comme la CDB. Dans le Programme de travail sur les aires protégées de la $\mathrm{CDB}$, par exemple, les Etats signataires se sont engagés à développer des systèmes d'évaluation de l'efficacité de la gestion et de remettre des rapports sur 30 pour cent de leurs aires protégées d'ici 2010.

L'évaluation de l'efficacité de la gestion peut :

- Permettre et encourager une approche adaptative de la gestion ;

- Aider à réaliser une allocation efficace des ressources ;

- Encourager la responsabilité et la transparence ;

- Impliquer la communauté et gagner du soutien pour les aires protégées.

La diversité des raisons de faire une évaluation, combinée à la grande variété des aires protégées - avec des valeurs et des objectifs, des situations culturelles, des régimes de gestion et des défis différents - signifie qu'il n'est pas pratique de mettre au point un instrument d'évaluation unique. C'est pourquoi la CMAP/UICN a décidé de développer un cadre commun ( $2^{\mathrm{eme} e}$ édition, Hockings et al. 2006 - version française disponible) qui fournit une base cohérente pour concevoir des systèmes d'évaluation, donne des orientations au sujet de ce qu'il faut évaluer et fournit des critères généraux pour l'évaluation. Le processus d'évaluation recommandé par l'UICN est résumé au Tableau 18 ci-dessous. Sur la base de ce cadre, toute une gamme d' "outils " d'évaluation peuvent servir à mener des évaluations à différentes échelles et profondeurs.

Tableau 18. Eléments du cadre de la CMAP pour évaluer l'efficacité de gestion d'aires protégées

\begin{tabular}{|c|c|c|c|c|c|c|}
\hline & \multicolumn{2}{|c|}{ Conception } & \multicolumn{2}{|c|}{ Pertinence/Justesse } & \multicolumn{2}{|c|}{ Production de résultats } \\
\hline & Contexte & Planification & Intrants & Processus & Extrants & Résultats \\
\hline $\begin{array}{l}\text { Point } \\
\text { focal de } \\
\text { l'évaluation }\end{array}$ & $\begin{array}{l}\text { Importance } \\
\text { Menaces } \\
\text { Politique } \\
\text { environnementale }\end{array}$ & $\begin{array}{l}\text { Conception et } \\
\text { planification }\end{array}$ & $\begin{array}{l}\text { Ressources } \\
\text { nécessaires } \\
\text { pour mener la } \\
\text { gestion }\end{array}$ & $\begin{array}{l}\text { Evaluation } \\
\text { de la façon } \\
\text { dont la } \\
\text { gestion est } \\
\text { menée }\end{array}$ & $\begin{array}{l}\text { Mise en } \\
\text { œuvre des } \\
\text { programmes } \\
\text { et activités } \\
\text { de gestion }\end{array}$ & $\begin{array}{l}\text { Mesure } \\
\text { dans } \\
\text { laquelle les } \\
\text { objectifs } \\
\text { sont atteints }\end{array}$ \\
\hline $\begin{array}{l}\text { Critères } \\
\text { évalués }\end{array}$ & $\begin{array}{l}\text { Valeurs } \\
\text { Menaces } \\
\text { Vulnérabilité } \\
\text { Parties prenantes } \\
\text { Contexte national }\end{array}$ & $\begin{array}{l}\text { Législation et } \\
\text { politique } \\
\text { Conception } \\
\text { du système } \\
\text { Planification } \\
\text { de la gestion }\end{array}$ & $\begin{array}{l}\text { Adéquation } \\
\text { des ressources } \\
\text { disponibles } \\
\text { pour } \\
\text { l'administration } \\
\text { en charge }\end{array}$ & $\begin{array}{l}\text { Pertinence } \\
\text { des } \\
\text { processus } \\
\text { de gestion }\end{array}$ & $\begin{array}{l}\text { Résultats } \\
\text { des activités } \\
\text { de gestion }\end{array}$ & $\begin{array}{l}\text { Effets de la } \\
\text { gestion par } \\
\text { rapport aux } \\
\text { objectifs }\end{array}$ \\
\hline
\end{tabular}

De nombreux systèmes d'évaluation de l'efficacité de la gestion ont été développés au cours des 10 à 15 dernières années mais beaucoup d'entre eux n'ont été appliqués qu’à quelques aires protégées. Plus de 90 pour cent des évaluations de sites ont été faites en utilisant des systèmes compatibles avec le cadre de la CMAP/UICN. Cela signifie qu'elles partagent une approche sous-jacente commune et des critères en grande partie similaires, même si les indicateurs et les méthodes d'évaluation varient. Les systèmes peuvent être généralement divisés en deux types principaux : (1) les systèmes qui ont recours surtout aux connaissances des experts et (2) les systèmes qui utilisent le suivi des données, les enquêtes auprès des parties prenantes et d'autres sources de données quantitatives ou qualitatives. Certains systèmes d'évaluation combinent les deux approches d'une évaluation en fonction de l'aspect de la gestion qui est évalué. Les systèmes ayant recours à la connaissance d'experts 
utilisent généralement une approche par questionnaire qui demande aux gens qui ont une connaissance poussée de l'aire protégée et de sa gestion de coter les différents aspects de la gestion ou de désigner des caractéristiques du site, telles que la nature et l'importance des valeurs de l'aire protégée et des menaces. Ces évaluations peuvent être étayées par une base considérable de connaissances consistant en résultats du suivi et de la recherche réalisés sur le site. Cette approche d'évaluation est souvent appliquée lorsque l'on évalue la gestion d'un grand nombre d'aires protégées, souvent celle de toutes les aires protégées d'un pays, parce qu'elle est plus rapide et exige moins de ressources que l'approche par suivi continu.

\section{Relation entre évaluation et assignation d'une catégorie}

L'évaluation peut couvrir deux aspects différents des aires protégées :

- Déterminer si les objectifs choisis pour l'aire protégée correspondent à la catégorie assignée. Ceci relève d'un intérêt plus quacadémique si la politique ou la législation nationale lie la prise de décisions (concernant, par exemple, le financement, l'utilisation admissible des terres, les droits de chasse, etc.) à la désignation de la catégorie.

- Savoir si ces objectifs sont réellement atteints.

Le premier aspect est, à la base, une évaluation de l'intention de la gestion. La raison d'être de telles évaluations n'est pas d'évaluer l'efficacité de gestion mais de clarifier les objectifs exprimés et atteints pour la gestion. Une telle approche a été développée par la CMAP/UICN en Europe et a servi à " certifier " qu'une aire protégée est bien assignée à la catégorie d'aire protégée correcte (selon la législation et les réglementations en vigueur) et à voir si le site est géré en accord avec des objectifs de gestion correspondant à cette catégorie. Jusqu'ici, il n'existe pas de méthodologie écrite, et le système est en développement. Il se concentre particulièrement sur les deux premiers éléments du cadre de la CMAP - le contexte et la planification - et à peine sur les deux derniers, les extrants et les résultats.

Le second aspect examine plus attentivement si ces objectifs ont été atteints en pratique. Ces objectifs sont d'habitude spécifiés au niveau national dans la législation pertinente ou un autre système de gouvernance (p.ex. par l'autorité traditionnelle pour une aire conservée par la communauté) qui donne une orientation générale à la gestion du site. Par exemple, la désignation en tant qu'aire protégée de catégorie II signifie que l'aire doit d'abord être gérée pour la conservation de sa biodiversité, sans utilisation extractive des ressources, ou très limitée. Dans certains cas, les gestionnaires peuvent avoir des difficultés à gérer le site dans le strict respect de ces objectifs. Il est convenu que les résultats d'une évaluation de l'efficacité de gestion ne doivent pas être utilisés comme base pour attribuer ou pour changer la catégorie à laquelle une aire protégée est assignée. Ainsi, par exemple, la réponse appropriée à une évaluation de l'efficacité de la gestion qui révèle un échec du contrôle de l'exploitation illégale des ressources dans une aire protégée de catégorie II n'est pas de faire passer le site dans la catégorie $\mathrm{V}$ (qui permet un certain niveau d'utilisation durable des ressources), mais plutôt de chercher à adapter la gestion pour qu'elle atteigne plus efficacement les objectifs de gestion légalement spécifiés.

A l'avenir, l'UICN va examiner la demande pour une évaluation plus rigoureuse de l'efficacité dans le contexte du système de catégories et en étudier les implications pratiques. 



\section{Annexe. Typologie et glossaire}

Les utilisateurs vont lire ces lignes directrices ligne par ligne, en essayant de porter des jugements difficiles, en travaillant dans une langue souvent différente de la leur. Les lignes directrices doivent donc être aussi claires que possible, mais la précision est rendue plus difficile du fait que de nombreux mots utilisés dans le domaine de l'écologie et de la conservation restent mal définis et qu'ils font l'objet de multiples interprétations.
Le glossaire du Tableau 19 est proposé ici pour apporter de la clarté et il faudrait s'en servir en même temps que la définition et la description des catégories qui suivent. Les sources utilisées se sont, autant que possible, inspirées des définitions antérieures de l'UICN et de celles de la CDB, et elles devraient donc être familières aux gouvernements et à ceux qui utilisent les catégories.

Tableau 19. Définitions des termes utilisés dans les lignes directrices

\begin{tabular}{|c|c|c|}
\hline Terme & Définition & Source et remarques \\
\hline $\begin{array}{l}\text { Agrobiodiversité } \\
\text { (Diversité } \\
\text { biologique } \\
\text { agricole) }\end{array}$ & $\begin{array}{l}\text { Inclut les plantes sauvages étroitement liées aux } \\
\text { cultures (parentes sauvages de variétés cultivées), } \\
\text { des plantes cultivées (races locales) et des variétés } \\
\text { de bétail domestique. L'agrobiodiversité peut être } \\
\text { un objectif d'aires protégées pour les espèces } \\
\text { parentes de plantes cultivées, pour des races locales } \\
\text { traditionnelles et menacées, particulièrement celles } \\
\text { qui dépendent de pratiques culturales traditionnelles ; } \\
\text { et/ou des races de bétail traditionnelles et menacées, } \\
\text { spécialement si elles dépendent de systèmes de } \\
\text { gestion culturale traditionnels qui sont compatibles } \\
\text { avec la « biodiversité sauvage ». }\end{array}$ & $\begin{array}{l}\text { Source : Amend, T., J. Brown, A. } \\
\text { Kothari, A. Phillips et S. Stolton (Eds). } \\
\text { 2008. Protected Landscapes and } \\
\text { Agrobiodiversity Values. Volume } 1 \text { dans } \\
\text { la série Values of Protected Landscapes } \\
\text { and Seascapes. Heidelberg: Kasparek } \\
\text { Verlag, pour l'IUCN et la GTZ. }\end{array}$ \\
\hline $\begin{array}{l}\text { Aire conservée } \\
\text { par la } \\
\text { communauté }\end{array}$ & $\begin{array}{l}\text { Ecosystèmes naturels et modifiés, comprenant } \\
\text { une biodiversité importante, des services } \\
\text { écologiques et des valeurs culturelles, conservés } \\
\text { volontairement par des peuples autochtones et } \\
\text { des communautés locales et nomades par des lois } \\
\text { coutumières ou d'autres moyens efficaces. }\end{array}$ & $\begin{array}{l}\text { Source : Borrini-Feyerabend, G., A. } \\
\text { Kothari et G. Oviedo. 2004. Indigenous } \\
\text { and Local Communities and Protected } \\
\text { Areas: Towards Equity and Enhanced } \\
\text { Conservation. } N^{\circ} 11 \text { de la Série } \\
\text { "Lignes directrices sur les meilleures } \\
\text { pratiques pour les aires protégées ». } \\
\text { Gland et Cambridge: UICN. }\end{array}$ \\
\hline $\begin{array}{l}\text { Aire protégée } \\
\text { à gouvernance } \\
\text { partagée }\end{array}$ & $\begin{array}{l}\text { Aire protégée créée par un gouvernement } \\
\text { où le pouvoir décisionnel, la responsabilité et } \\
\text { l'imputabilité sont partagés entre les agences } \\
\text { gouvernementales et d'autres parties prenantes, } \\
\text { en particulier les peuples autochtones et les } \\
\text { communautés locales et nomades qui dépendent, } \\
\text { culturellement ou pour leurs moyens de } \\
\text { subsistance, de cette aire. }\end{array}$ & $\begin{array}{l}\text { Source : Borrini-Feyerabend, G., A. } \\
\text { Kothari et G. Oviedo. 2004. Indigenous } \\
\text { and Local Communities and Protected } \\
\text { Areas: Towards Equity and Enhanced } \\
\text { Conservation. } \mathrm{N}^{\circ} 11 \text { de la Série } \\
\text { « Lignes directrices sur les meilleures } \\
\text { pratiques pour les aires protégées ». } \\
\text { Gland et Cambridge: UICN. }\end{array}$ \\
\hline Biome & $\begin{array}{l}\text { Majeure partie d'un environnement vivant dans une } \\
\text { région particulière (comme les forêts de conifères } \\
\text { ou les prairies), caractérisée par sa végétation } \\
\text { particulière et maintenue en grande partie par des } \\
\text { conditions climatiques locales. }\end{array}$ & $\begin{array}{l}\text { Source : Tiré du Glossaire (en anglais) } \\
\text { de la biodiversité de la CDB : CBD } \\
\text { Communication, Education and Public } \\
\text { Awareness (CEPA) Toolkit: http://www. } \\
\text { cbd.int/cepa/toolkit/2008/cepa/index.htm }\end{array}$ \\
\hline Cadre & $\begin{array}{l}\text { Une structure de haut niveau qui établit une raison } \\
\text { d'être et une direction communes pour des plans et } \\
\text { des programmes. }\end{array}$ & $\begin{array}{l}\text { Source : The CBD Communication, } \\
\text { Education and Public Awareness } \\
\text { (CEPA) Toolkit: http://www.cbd.int/cepa/ } \\
\text { toolkit/2008/cepa/index.htm } \\
\text { Cette définition vient du Glossaire } \\
\text { CEPA; qui est une version mise à } \\
\text { jour d'un glossaire de communication } \\
\text { développé par l'IUCN CEC Product } \\
\text { Group on Corporate Communication, } \\
\text { édité par Frits Hesselink en } 2003 \text {. }\end{array}$ \\
\hline
\end{tabular}


Tableau 19. Définitions des termes utilisés dans les lignes directrices (suite)

\begin{tabular}{|c|c|c|}
\hline Terme & Définition & Source et remarques \\
\hline $\begin{array}{l}\text { Conservation } \\
\text { in-situ }\end{array}$ & $\begin{array}{l}\text { La conservation des écosystèmes et des habitats } \\
\text { naturels et la sauvegarde et la reconstitution de } \\
\text { populations viables d'espèces dans leur milieu } \\
\text { naturel et, dans le cas des espèces domestiquées } \\
\text { et cultivées, dans le milieu où se sont développés } \\
\text { leurs caractères distinctifs. }\end{array}$ & $\begin{array}{l}\text { Source : CDB, Article 2. Emploi des } \\
\text { termes http://www.cbd.int/doc/legal/ } \\
\text { cbd-un-fr.pdf } \\
\text { Traductions : Anglais, arabe, chinois, } \\
\text { espagnol, français et russe. }\end{array}$ \\
\hline Corridor & $\begin{array}{l}\text { Façon de sauvegarder une connectivité écologique } \\
\text { ou environnementale vitale en préservant des } \\
\text { liaisons physiques entre des zones noyaux. }\end{array}$ & $\begin{array}{l}\text { Source : Bennett, G. et K.J. Mulongoy. } \\
\text { 2006. Review of experience with } \\
\text { ecological networks, corridors and buffer } \\
\text { zones. Technical Series no. 23. Montréal: } \\
\text { Secrétariat de la CDB (SCDB). }\end{array}$ \\
\hline $\begin{array}{l}\text { Diversité } \\
\text { biologique }\end{array}$ & $\begin{array}{l}\text { La variabilité des organismes vivants de toute } \\
\text { origine, y compris, entre autres, les écosystèmes } \\
\text { terrestres, marins et autres écosystèmes } \\
\text { aquatiques et les complexes écologiques dont } \\
\text { ils font partie ; cela comprend la diversité au sein } \\
\text { des espèces, entre espèces ainsi que celle des } \\
\text { écosystèmes. }\end{array}$ & $\begin{array}{l}\text { Source : CDB, Article 2. Emploi des } \\
\text { termes http://www.cbd.int/doc/legal/ } \\
\text { cbd-un-fr.pdf } \\
\text { Traductions : Texte disponible en } \\
\text { anglais, arabe, chinois, espagnol, } \\
\text { français et russe sur le site de la CDB. }\end{array}$ \\
\hline Ecosystème & $\begin{array}{l}\text { Un complexe dynamique formé de communautés } \\
\text { de plantes, d'animaux et de micro-organismes } \\
\text { et de leur environnement non vivant qui, par leur } \\
\text { interaction, forment une unité fonctionnelle. }\end{array}$ & $\begin{array}{l}\text { Source : CDB, Article 2. Emploi des } \\
\text { termes http://www.cbd.int/doc/legal/ } \\
\text { cbd-un-fr.pdf } \\
\text { Traductions : Anglais, arabe, chinois, } \\
\text { espagnol, français et russe. }\end{array}$ \\
\hline $\begin{array}{l}\text { Efficacité de } \\
\text { gestion }\end{array}$ & $\begin{array}{l}\text { L'évaluation de la mesure dans laquelle les aires } \\
\text { protégées sont bien gérées - et tout d'abord de } \\
\text { la mesure dans laquelle la gestion protège les } \\
\text { valeurs et atteint les buts et les objectifs des aires } \\
\text { protégées. }\end{array}$ & $\begin{array}{l}\text { Source: Hockings, M., S. Stolton, F. } \\
\text { Leverington, N. Dudley et J. Courrau. } \\
2006 \text {. Evaluation de l'efficacité : Un } \\
\text { cadre pour l'évaluation de l'efficacité } \\
\text { de la gestion des aires protégées, } 2^{\text {ème }} \\
\text { édition. } \\
\mathrm{N}^{\circ} 14 \text { de la Série « Lignes directrices } \\
\text { sur les meilleures pratiques pour les } \\
\text { aires protégées ». Gland et Cambridge: } \\
\text { UICN. } \\
\text { Traductions : Anglais, espagnol et } \\
\text { français. }\end{array}$ \\
\hline Géodiversité & $\begin{array}{l}\text { La diversité de minéraux, de roches (solides ou } \\
\text { meubles), de fossiles, de modelés, de sédiments } \\
\text { et de sols, et de processus naturels qui constituent } \\
\text { la topographie, le paysage et la structure sous- } \\
\text { jacente de la terre. }\end{array}$ & $\begin{array}{l}\text { Source : McKirdy, A., J. Gordon et R. } \\
\text { Crofts. 2007. Land of Mountain and } \\
\text { Flood: the geology and landforms of } \\
\text { Scotland. Edinburgh: Birlinn. }\end{array}$ \\
\hline Gouvernance & $\begin{array}{l}\text { Dans le contexte des aires protégées, la } \\
\text { gouvernance a été définie comme «les } \\
\text { interactions entre structures, processus et } \\
\text { traditions qui déterminent comment le pouvoir est } \\
\text { exercé, comment les décisions concernant des } \\
\text { questions d'intérêt public sont prises et comment } \\
\text { les citoyens ou autres parties prenantes ont leur } \\
\text { mot à dire ». Les accords de gouvernance sont } \\
\text { exprimés dans des cadres légaux et politiques, des } \\
\text { stratégies et des plans de gestion; ils comprennent } \\
\text { les accords organisationnels pour la mise en } \\
\text { pratique des politiques et des plans et le suivi des } \\
\text { performances. La gouvernance couvre les règles } \\
\text { en matière de prise de décisions, y compris pour } \\
\text { savoir qui a accès à l'information et qui participe } \\
\text { au processus de prise de décisions, ainsi qu'aux } \\
\text { décisions elles-mêmes. }\end{array}$ & $\begin{array}{l}\text { Source : Borrini-Feyerabend, G., A. } \\
\text { Kothari et G. Oviedo. 2004. Indigenous } \\
\text { and Local Communities and Protected } \\
\text { Areas: Towards Equity and Enhanced } \\
\text { Conservation. N }{ }^{\circ} 11 \text { de la Série } \\
\text { « Lignes directrices sur les meilleures } \\
\text { pratiques pour les aires protégées ». } \\
\text { Gland et Cambridge: UICN. }\end{array}$ \\
\hline
\end{tabular}


Tableau 19. Définitions des termes utilisés dans les lignes directrices (suite)

\begin{tabular}{|c|c|c|}
\hline Terme & Définition & Source et remarques \\
\hline $\begin{array}{l}\text { Parties } \\
\text { prenantes }\end{array}$ & $\begin{array}{l}\text { Les personnes ou organisations qui sont vitales } \\
\text { pour la réussite ou l'échec d'une organisation } \\
\text { ou un projet qui doit atteindre ses objectifs. Les } \\
\text { parties prenantes principales sont (a) celles } \\
\text { dont on a besoin pour des autorisations, des } \\
\text { approbations et un soutien financier et (b) celles } \\
\text { qui sont directement affectées par les activités de } \\
\text { l'organisation ou du projet. Les parties prenantes } \\
\text { secondaires sont celles qui sont affectées } \\
\text { indirectement. Les parties prenantes tertiaires } \\
\text { sont celles qui ne sont ni affectées, ni impliquées, } \\
\text { mais qui peuvent influencer les opinions de façon } \\
\text { positive ou négative. }\end{array}$ & $\begin{array}{l}\text { Source: The CBD Communication, } \\
\text { Education and Public Awareness } \\
\text { (CEPA) Toolkit: http://www.cbd.int/cepa/ } \\
\text { toolkit/2008/cepa/index.htm } \\
\text { Cette définition vient du glossaire } \\
\text { du CEPA qui est une version mise à } \\
\text { jour d'un glossaire de communication } \\
\text { développé par l'IUCN CEC Product } \\
\text { Group on Corporate Communication, } \\
\text { édité par Frits Hesselink en } 2003 \text {. }\end{array}$ \\
\hline $\begin{array}{l}\text { Populations } \\
\text { autochtones et } \\
\text { tribales }\end{array}$ & $\begin{array}{l}\text { (a) Des peuples tribaux dans les pays } \\
\text { indépendants qui se distinguent des autres } \\
\text { secteurs de la communauté nationale par leurs } \\
\text { conditions sociales, culturelles et économiques et } \\
\text { qui sont régis totalement ou partiellement par des } \\
\text { coutumes ou des traditions qui leur sont propres ou } \\
\text { par une législation spéciale ; } \\
\text { b) Des peuples dans les pays indépendants qui } \\
\text { sont considérés comme indigènes du fait qu'ils } \\
\text { descendent des populations qui habitaient le pays, } \\
\text { ou une région géographique à laquelle appartient } \\
\text { le pays, à l'époque de la conquête ou de la } \\
\text { colonisation ou de l'établissement des frontières } \\
\text { actuelles de l'Etat, et qui, quel que soit leur statut } \\
\text { juridique, conservent leurs institutions sociales, } \\
\text { économiques, culturelles et politiques propres ou } \\
\text { certaines d'entre elles. }\end{array}$ & $\begin{array}{l}\text { Source: Définition appliquée à } \\
\text { l'Organisation mondiale du travail (OIT) } \\
\text { Convention (No. 169) concernant les } \\
\text { populations autochtones et tribales } \\
\text { dans des pays indépendants. Les } \\
\text { populations autochtones insistent aussi } \\
\text { sur le fait qu'il y a un degré d'auto- } \\
\text { définition dans la définition de ce qui } \\
\text { constitue une population autochtone ou } \\
\text { tribale spécifique. }\end{array}$ \\
\hline $\begin{array}{l}\text { Qualité de la } \\
\text { gouvernance }\end{array}$ & $\begin{array}{l}\text { Comment une aire protégée est gouvernée - la } \\
\text { mesure dans laquelle elle répond aux principes et } \\
\text { aux critères de « bonne gouvernance » identifiés } \\
\text { et choisis par les peuples, les communautés et les } \\
\text { gouvernements concernés (partie de leur sens de } \\
\text { la moralité, de l'identité culturelle et de la fierté) et } \\
\text { généralement liés aux principes épousés par les } \\
\text { organisations et les conventions internationales. }\end{array}$ & $\begin{array}{l}\text { Source : Borrini-Feyerabend, G. } 2004 . \\
\text { "Governance of protected areas, } \\
\text { participation and equity", pp. 100-105 } \\
\text { dans Secretariat of the Convention on } \\
\text { Biological Diversity, Biodiversity Issues } \\
\text { for Consideration in the Planning, } \\
\text { Establishment and Management } \\
\text { of Protected Areas and Networks. } \\
\text { Technical Series no. 15. Montréal : } \\
\text { SCDB. }\end{array}$ \\
\hline $\begin{array}{l}\text { Services } \\
\text { écosystémiques }\end{array}$ & $\begin{array}{l}\text { Les bienfaits que les écosystèmes procurent } \\
\text { aux humains. Ceux-ci comprennent des services } \\
\text { d'approvisionnement, comme la nourriture et l'eau; } \\
\text { des services régulateurs comme la régulation des } \\
\text { inondations, de la sécheresse, de la dégradation } \\
\text { des sols et des maladies; des services de soutien, } \\
\text { comme la formation des sols et le cycle des } \\
\text { nutriments, et des services culturels tels que les } \\
\text { avantages récréatifs, spirituels, religieux et autres } \\
\text { avantages non matériels. }\end{array}$ & $\begin{array}{l}\text { Source : Hassan, R., R. Scholes et } \\
\text { N. Ash (Eds). 2005. Ecosystems and } \\
\text { Human Well-Being: Current State and } \\
\text { Trends: Findings of the Condition and } \\
\text { Trends Working Group v. } 1 \text { (Millennium } \\
\text { Ecosystem Assessment). Washington } \\
\text { DC: Island Press. } \\
\text { Définitions (ang.) dans: Chapitre 1: MA } \\
\text { Conceptual Framework. }\end{array}$ \\
\hline Site sacré & $\begin{array}{l}\text { Une aire d'importance spirituelle spéciale pour des } \\
\text { peuples et des communautés. }\end{array}$ & \\
\hline $\begin{array}{l}\text { Sites naturels } \\
\text { sacrés }\end{array}$ & $\begin{array}{l}\text { Des aires terrestres ou aquatiques qui ont une } \\
\text { importance spirituelle pour des populations ou des } \\
\text { communautés. }\end{array}$ & $\begin{array}{l}\text { Source: Wild, R. et C. McLeod. } 2008 . \\
\text { Sacred Natural Sites: Guidelines for } \\
\text { Protected Area Managers. N } 16 \text { de } \\
\text { la Série « Lignes directrices sur les } \\
\text { meilleures pratiques pour les aires } \\
\text { protégées ». Gland et Cambridge: UICN. }\end{array}$ \\
\hline
\end{tabular}


Tableau 19. Définitions des termes utilisés dans les lignes directrices (suite)

\begin{tabular}{|c|c|c|}
\hline Terme & Définition & Source et remarques \\
\hline $\begin{array}{l}\text { Type de } \\
\text { gouvernance }\end{array}$ & $\begin{array}{l}\text { Les types de gouvernance sont définis sur la base } \\
\text { de « qui détient l'autorité et la responsabilité de la } \\
\text { gestion et peut être tenu pour responsable » pour } \\
\text { une aire protégée donnée. }\end{array}$ & $\begin{array}{l}\text { Source : Borrini-Feyerabend, G. } 2004 . \\
\text { "Governance of protected areas, } \\
\text { participation and equity", pp. 100-105 } \\
\text { in Secretariat of the Convention on } \\
\text { Biological Diversity, Biodiversity Issues } \\
\text { for Consideration in the Planning, } \\
\text { Establishment and Management of } \\
\text { Protected Areas and Networks. Technical } \\
\text { Series no. 15. Montréal: SCDB. }\end{array}$ \\
\hline $\begin{array}{l}\text { Utilisation } \\
\text { durable }\end{array}$ & $\begin{array}{l}\text { L'utilisation des éléments constitutifs de la } \\
\text { diversité biologique d'une manière et à un rythme } \\
\text { qui n'entraînent pas leur appauvrissement à } \\
\text { long terme et sauvegardent ainsi leur potentiel } \\
\text { pour satisfaire les besoins et les aspirations des } \\
\text { générations présentes et futures. } \\
\text { (Cette définition de la CDB est spécifique de } \\
\text { l'utilisation durable qui se rapporte à la biodiversité). }\end{array}$ & $\begin{array}{l}\text { Source : CBD, Article 2. Emploi des } \\
\text { termes http://www.cbd.int/doc/legal/ } \\
\text { cbd-un-fr.pdf } \\
\text { Traductions : Anglais, arabe, chinois, } \\
\text { espagnol, français et russe. }\end{array}$ \\
\hline Zone tampon & $\begin{array}{l}\text { Zones situées entre le noyau des aires } \\
\text { protégées et le paysage terrestre ou marin } \\
\text { environnant, qui protège le réseau d'influences } \\
\text { extérieures potentiellement négatives, et qui sont } \\
\text { essentiellement des zones de transition. }\end{array}$ & $\begin{array}{l}\text { Source : Bennett, G. et K.J. Mulongoy. } \\
\text { 2006. Review of experience with } \\
\text { ecological networks, corridors and } \\
\text { buffer zones. Technical Series no. } 23 \text {. } \\
\text { Montréal: SCDB. }\end{array}$ \\
\hline
\end{tabular}




\section{Références}

Bishop, K., N. Dudley, A. Phillips et S. Stolton. 2004. Speaking a Common Language - the uses and performance of the IUCN System of Management Categories for Protected Areas. Cardiff University, UICN et WCMC/PNUE.

Borrini-Feyerabend, G., A. Kothari et G. Oviedo. 2004. Indigenous and Local Communities and Protected Areas: Towards equity and enhanced conservation. № 11 de la Série «Lignes directrices sur les meilleures pratiques pour les aires protégées ». Gland et Cambridge: UICN.

Bridgewater, P., A. Phillips, M. Green et B. Amos. 1996. Biosphere Reserves and the IUCN System of Protected Area Management Categories. Canberra: Australian Nature Conservation Agency.

Brockman, C.F. 1962. "Supplement to the Report to the Committee on Nomenclature". In: Adams, A.B. (Ed.) First World Conference on National Parks. Washington, DC: National Park Service.

CBD. 2004. http://www.cbd.int/programmes/cross-cutting/ecosystem/default.shtml. Accessed 24 August 2007.

Chape, S., S. Blyth, L. Fish, P. Fox et M. Spalding. (Eds). 2003. 2003 United Nations List of Protected Areas. Gland et Cambridge: UICN et WCMC/PNUE.

Davey, A.G. 1998. National System Planning for Protected Areas. № 1 de la Série «Lignes directrices sur les meilleures pratiques pour les aires protégées ». Gland et Cambridge: UICN.

Day, J. 2002. "Zoning: Lessons from the Great Barrier Marine Park". Ocean and Coastal Management 45: 139-156.

Dillon, B. 2004. "The Use of the Categories in National and International Legislation and Policy". PARKS 14(3): 15-22.

Dudley, N., L. Higgins-Zogib et S. Mansourian. 2006. Beyond Belief: Linking faiths and protected area networks to support biodiversity conservation. Gland et Bath: WWF International et Alliance on Religions and Conservation.

Dudley, N. et J. Parrish. 2006. Closing the Gap: Creating ecologically representative protected area systems. Technical Series no. 24. Montreal: SCBD.

Dudley, N. et A. Phillips. 2006. Forests and Protected Areas: Guidance on the use of the IUCN protected area management categories. No 12 de la Série "Lignes directrices sur les meilleures pratiques pour les aires protégées ». Gland et Cambridge: UICN.

Dudley, N. et S. Stolton. 2003. "Ecological and socio-economic benefits of protected areas in dealing with climate change". En: Hansen, L.J., J.L. Biringer et J.R. Hoffman (Eds) Buying Time: A user's guide to building resistance and resilience to climate change in natural systems, pp. 217-233. Washington, DC: WWF US.

Eidsvik, H. 1990. A Framework for Classifying Terrestrial and Marine Protected Areas. Based on the Work of the CNPPA Task Force on Classification, IUCN/CNPPA. Unpublished.

Elliott, H.B. (Ed). 1974. Second World Conference on National Parks, Proceedings. Morges: UICN.

EUROPARC et IUCN. 1999. Guidelines for Protected Area Management Categories - Interpretation and Application in Europe. Grafenau: EUROPARC.

Graham, J., B. Amos et T. Plumptre. 2003. Principles for Good Governance in the $21^{\text {st }}$ Century. Policy Brief Number 15. Ottawa: Institute on Governance.

Hockings, M., S. Stolton, F. Leverington, N. Dudley et J. Courrau. 2006. Evaluating Effectiveness: A framework for assessing management effectiveness of protected areas. 2nd edition. № 14 de la Série «Lignes directrices sur les meilleures pratiques pour les aires protégées ». Gland et Cambridge: IUCN. [Disponible en français - 2008].

Holdaway, E. Non-daté. Making the Connection between Land and Sea: The place for coastal protected landscapes in the marine environment. Wadebridge et Bangor: EUROPARC Atlantic Isles et le Countryside Council for Wales.

Holdgate, M. 1999. The Green Web. London: Earthscan.

IUCN. 1974. Classification and Use of Protected Natural and Cultural Areas. IUCN Occasional Paper No. 4. Morges: UICN.

IUCN. 1978. Categories, Objectives and Criteria: Final Report of the Committee and Criteria of the CNPPA/IUCN. Morges: UICN.

IUCN/WCMC. 1994. Guidelines for Protected Area Management Categories. Gland et Cambridge: UICN.

IUCN. 2004. PARKS 14. (includes 10 papers).

Kelleher, G. 2002. Guidelines for Marine Protected Areas. № 3 de la Série « Lignes directrices sur les meilleures pratiques pour les aires protégées ». Gland et Cambridge: UICN.

Palumbi, S.R. 2001. "The ecology of marine protected areas". En: Bertness, M.D, S.M. Gaines et M.E. Hixon (Eds). Marine Community Ecology, pp.509-530. Sunderland, MA: Sinauer Associates.

Phillips, A. 2002. Management Guidelines for IUCN Category V Protected Areas: Protected Landscapes/Seascapes. № 9 de la Série «Lignes directrices sur les meilleures pratiques pour les aires protégées ». Gland et Cambridge: UICN. 
Phillips, A. 2007. "A short history of the international system of protected area management categories". Paper prepared for the WCPA Task Force on protected area categories.

Sandwith. T., C. Shine, L. Hamilton et D. Sheppard. 2001. Transboundary Protected Areas for Peace and Cooperation. No 7 de la Série « Lignes directrices sur les meilleures pratiques pour les aires protégées ». Gland et Cambridge: UICN.

Stolton, S., N. Dudley et J. Randall. 2008. Natural Security: Protected areas and hazard mitigation. The Arguments for Protection Series. Gland: WWF International.

Sulu, R., R. Cumming, L. Wantiez, L. Kumar, A. Mulipola, M. Lober, S. Sauni, T. Poulasi et K. Pakoa. 2002. "Status of Coral Reefs in the Southwest Pacific Region to 2002: Fiji, Nauru, New Caledonia, Samoa, Solomon Islands, Tuvalu and Vanuatu". En: Wilkinson, C.R. (Ed.) Status of Coral Reefs of the World 2002. Townsville, Queensland: Australian Institute of Marine Science. 


\section{UICN}

UNION INTERNATIONALE POUR LA CONSERVATION DE LA NATURE

SIĖGE MONDIAL

Rue Mauverney 28

1196 Gland

Suisse

mail@iucn.org

Tél +41229990000

Fax +41229990002

www.iucn.org

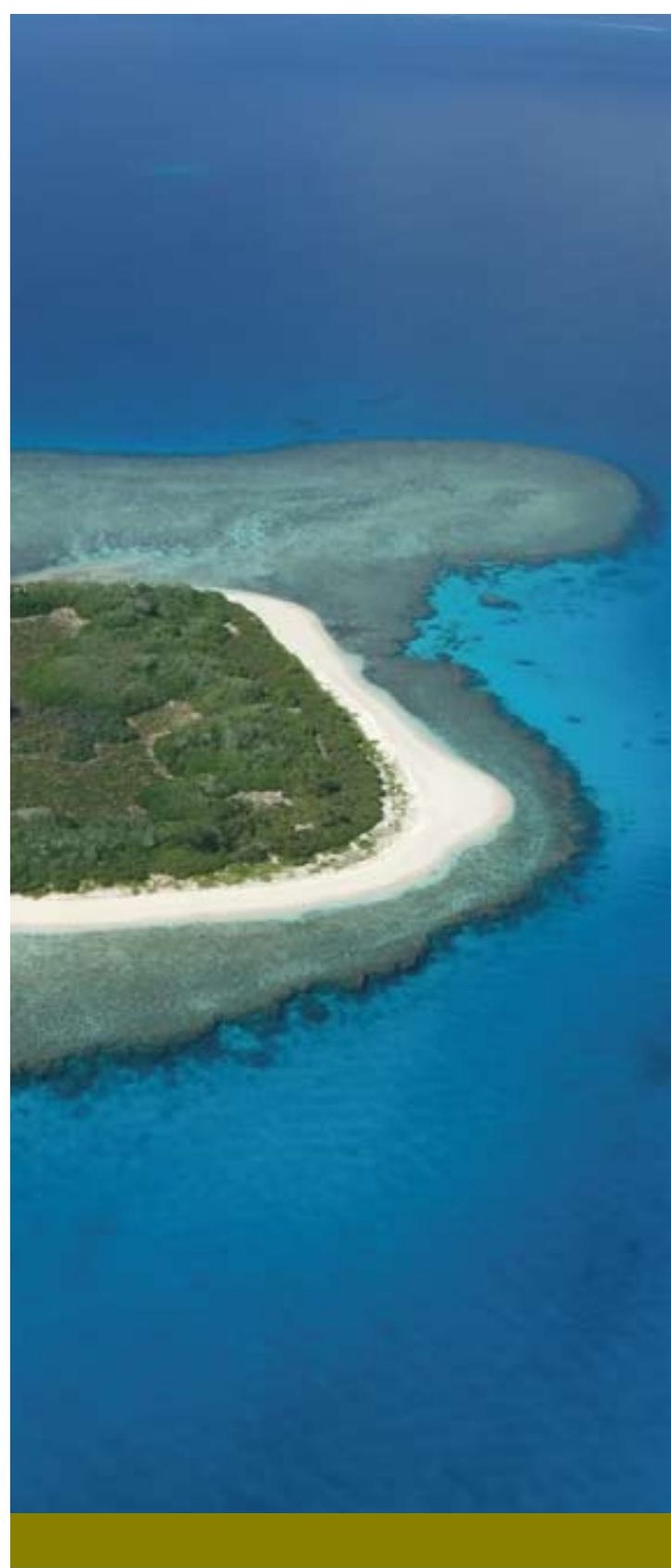

